\title{
Global seismology of the Sun
}

\author{
Sarbani Basu' ${ }^{1}$
}

Received: 16 September 2014 / Accepted: 1 June 2016 / Published online: 8 August 2016

(C) The Author(s) 2016. This article is published with open access at Springerlink.com

\begin{abstract}
The seismic study of the Sun and other stars offers a unique window into the interior of these stars. Thanks to helioseismology, we know the structure of the Sun to admirable precision. In fact, our knowledge is good enough to use the Sun as a laboratory. We have also been able to study the dynamics of the Sun in great detail. Helioseismic data also allow us to probe the changes that take place in the Sun as solar activity waxes and wanes. The seismic study of stars other than the Sun is a fairly new endeavour, but we are making great strides in this field. In this review I discuss some of the techniques used in helioseismic analyses and the results obtained using those techniques. I focus on results obtained with global helioseismology, i.e., the study of the Sun using its normal modes of oscillation. I also briefly touch upon asteroseismology, the seismic study of stars other than the Sun, and discuss how seismic data of others stars are interpreted.
\end{abstract}

Keywords Sun: interior $\cdot$ Sun: helioseismology $\cdot$ Sun: rotations ·

Stars: asteroseismology

\section{Contents}

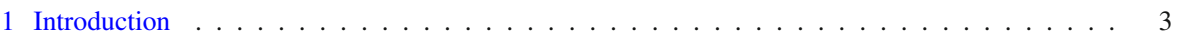

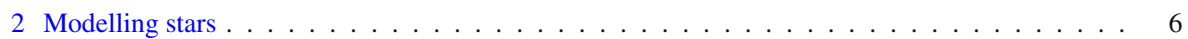

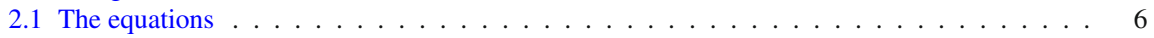

2.2 Inputs to stellar models . . . . . . . . . . . . . . . . . . . . . . . . 10

2.2 .1 The equation of state . . . . . . . . . . . . . . . . . . . . . 10

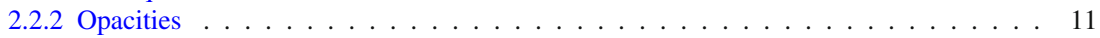

Sarbani Basu

sarbani.basu@yale.edu

1 Department of Astronomy, Yale University, PO Box 208101, New Haven, CT 06520-8101, USA 
2.2 .3 Nuclear reaction rates . . . . . . . . . . . . . . . . . . . . . . . . . . . . . . . . . . . . . .

2.2 .4 Diffusion coefficients . . . . . . . . . . . . . . . . . . . . . . . . . . . . . . . . . . . .

2.2 .5 Atmospheres . . . . . . . . . . . . . . . . . . . . . . 11

2.3 The concept of "standard" solar models . . . . . . . . . . . . . . . . . . . . . 11

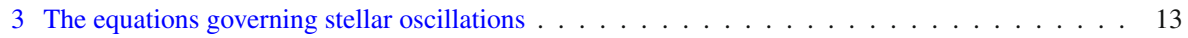

3.1 The spherically symmetric case . . . . . . . . . . . . . . . . . . . . . . . . . . . . . . . . . . . 15

3.2 Boundary conditions . . . . . . . . . . . . . . . . . . . . . . 18

3.3 Properties of stellar oscillations . . . . . . . . . . . . . . . . . . . . . 20

$3.3 .1 \mathrm{P}$ modes . . . . . . . . . . . . . . . . . . . . . . . . . . . . . . . . . . . . . . . 21

3.3 .2 G modes . . . . . . . . . . . . . . . . . . . . . . . . . . . . . . . . . . . . .

3.3 .3 Remaining issues . . . . . . . . . . . . . . . . . . . . . . . . . . . . . . . . 25

4 A brief account of the history of solar models . . . . . . . . . . . . . . . . . . . . 26

5 Frequency comparisons and the issue of the surface term' . . . . . . . . . . . . . . 28

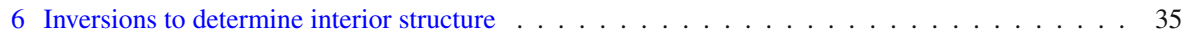

6.1 Asymptotic inversions . . . . . . . . . . . . . . . . . . . 36

6.2 Full inversions . . . . . . . . . . . . . . . . . . . . . . . . . . . . . . . . . . 37

6.2 .1 Inversions kernels . . . . . . . . . . . . . . . . . . . . . 39

6.2 .2 Taking care of the surface term . . . . . . . . . . . . . . . . . . 43

6.3 Inversion techniques . . . . . . . . . . . . . . . . . . . . . . . . . . . . . . . . . . 44

6.3 .1 The regularised least squares technique . . . . . . . . . . . . . . . . . . 45

Implementing RLS . . . . . . . . . . . . . . . . . . . . . . 46

6.3 .2 Optimally localised averages . . . . . . . . . . . . . . . . . . . . . 48

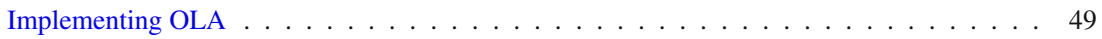

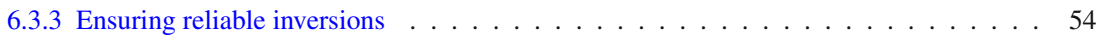

7 Results from structure inversions . . . . . . . . . . . . . . . . . . 56

7.1 Solar structure and the solar neutrino problem . . . . . . . . . . . . . . . 56

7.2 Some properties of the solar interior . . . . . . . . . . . . . . . . 58

7.2.1 The base of the convection zone . . . . . . . . . . . . . . . 58

7.2.2 The convection-zone helium abundance . . . . . . . . . . . . . . . . 61

7.3 Testing input physics . . . . . . . . . . . . . . . . . . . . . 62

7.4 Seismic models . . . . . . . . . . . . . . . . . . . . . . . . . . 66

7.5 The solar abundance issue . . . . . . . . . . . . . . . . . . . . . . . . . . . . . . . . . . . . . . 66

7.6 How well do we really know the structure of the Sun? . . . . . . . . . . . . . . . . . . . . 69

8 Departures from spherical symmetry . . . . . . . . . . . . . . . . . . 70

8.1 Solar rotation . . . . . . . . . . . . . . . . . . . . 71

8.2 Other deviations from spherical symmetry . . . . . . . . . . . . . . . . . . . . . . . . . . . . . . 73

8.2 .1 Magnetic fields . . . . . . . . . . . . . . . . . . . . . . 73

8.2 .2 Acoustic asphericity . . . . . . . . . . . . . . . . . . . 74

9 Solar-cycle related effects . . . . . . . . . . . . . . . . . . . . . . 75

9.1 Were there solar cycle-dependent structural changes in the Sun? . . . . . . . . . . . . 78

9.2 What do the even-order splittings tell us? . . . . . . . . . . . . . . . . . . . . 81

9.3 Changes in solar dynamics . . . . . . . . . . . . . . . . . . . . . . . . . . . . . . . . . . . . . . . 82

9.4 The "peculiar" solar cycle $24 \ldots \ldots \ldots \ldots$. . . . . . . . . . . . . . . . 85

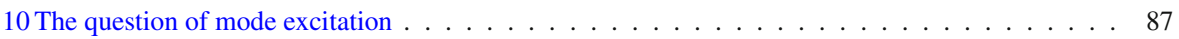

11 Seismology of other stars . . . . . . . . . . . . . . . . . . . . . . . 90

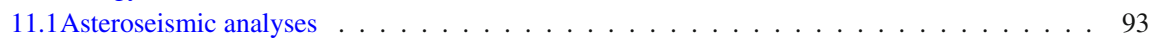

11.1.1Grid based modelling . . . . . . . . . . . . . . . . . . . . . . . . . . . . . . . 95

11.1.2Detailed modelling . . . . . . . . . . . . . . . . . . . . . . . . . . . . . . . . . 96

11.1.3Exploiting acoustic glitches . . . . . . . . . . . . . . . . . . . . . . . . . . . . . . . 98

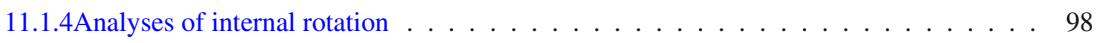

12 Concluding thoughts . . . . . . . . . . . . . . . . . . . . . . . . 99

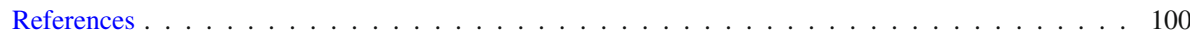




\section{Introduction}

The Sun and other stars oscillate in their normal modes. The analysis and interpretation of the properties of these modes in terms of the underlying structure and dynamics of the stars is referred to as global seismology. Global seismology has given us an unprecedented window into the structure and dynamics of the Sun and stars. Arthur Eddington began his book The Internal Constitution of the Stars lamenting the fact that the deep interior of the Sun and stars is more inaccessible than the depths of space since we so not have an "appliance" that can "... pierce through the outer layers of a star and test the conditions within". Eddington went on to say that perhaps the only way of probing the interiors of the Sun and stars is to use our knowledge of basic physics to determine what the structure of a star should be. While this is still the dominant approach in the field of stellar astrophysics, in global seismology we have the means of piercing the outer layers of a star to probe the structure within.

The type of stellar oscillations that are used in helio- and asteroseismic analyses have very low amplitudes. These oscillations are excited by the convective motions in the outer convection zones of stars. Such oscillations, usually referred to as solar-like oscillations, can for most purposes be described using the theory of linear, adiabatic, oscillations. The behaviour of the modes on the stellar surface is described in terms of spherical harmonics since these functions are a natural description of the normal modes of a sphere. The oscillations are labelled by three numbers, the radial order $n$, the degree $\ell$ and the azimuthal order $m$. The radial order $n$ can be any whole number and is the number of nodes in the radial direction. Positive values of $n$ are used to denote acoustic modes, i.e., the so-called p modes ( $p$ for pressure, since the dominant restoring force for these modes is provided by the pressure gradient). Negative values of $n$ are used to denote modes for which buoyancy provides the main restoring force. These are usually referred to as g modes (g for gravity). Modes with $n=0$ are the so-called fundamental or $\mathrm{f}$ modes. These are essentially surface gravity modes whose frequencies depend predominantly on the wave number and the surface gravity. The degree $\ell$ denotes the number of nodal planes that intersect the surface of a star and $m$ is the number of nodal planes perpendicular to equator.

For a spherically symmetric star, all modes with the same degree $\ell$ and order $n$ have the same frequency. Asphericities such as rotation and magnetic fields lift this degeneracy and cause 'frequency splitting' making the frequencies $m$-dependent. It is usual to express the frequency $v_{n \ell m}$ of a mode in terms of 'splitting coefficients':

$$
\frac{\omega_{n \ell m}}{2 \pi}=v_{n \ell m}=v_{n \ell}+\sum_{j=1}^{j_{\max }} a_{j}(n \ell) \mathcal{P}_{j}^{n \ell}(m),
$$

where, $a_{j}$ are the splitting or ' $a$ ' coefficients and $\mathcal{P}$ are suitable polynomials. For slow rotation the central frequency $v_{n \ell}$ depends only on structure, the odd-order $a$ coefficients depend on rotation, and the even-order $a$ coefficients depend on structural asphericities, magnetic fields, and second order effects of rotation.

The focus of this review is global helioseismology-its theoretical underpinnings as well as what it has taught us about the Sun. The last section is devoted to aster- 
oseismology; the theoretical background of asteroseismology is the same as that of helioseismology, but the diverse nature of different stars makes the field quite distinct. This is, of course, not the first review of helioseismology. To get an idea of the changing nature of the field, the reader is referred to earlier reviews by Thompson (1998b), Christensen-Dalsgaard (2002, 2004) and Gough (2013b), which along with descriptions of the then state-of-art of the field, also review the early history of helioseismology. Since this review is limited to global seismology only, readers are referred to the Living Reviews in Solar Physics contribution of Gizon and Birch (2005) for a review of local helioseismology.

Solar oscillations were first discovered by Leighton et al. (1962) and confirmed by Evans and Michard (1962). Later observations, such that those by Frazier (1968) indicated that the oscillations may not be mere surface phenomena. Subsequently Ulrich (1970) and Leibacher and Stein (1971) proposed that the observations could be interpreted as global oscillation modes and predicted that oscillations would form ridges in a wave-number v/s frequency diagram. The observations of Deubner (1975) indeed showed such ridges. Rhodes et al. (1977) reported similar observations. Neither the observations of Deubner (1975), nor those of Rhodes et al. (1977), could resolve individual modes of solar oscillations. Those had to wait for Claverie et al. (1979) who, using Doppler-velocity observations integrated over the solar disk, were able to resolve the individual modes of oscillations corresponding to the largest horizontal wavelengths, i.e., the truly global modes. They found a series of almost equidistant peaks in the power spectrum, just as was expected from theoretical models.

Helioseismology, the study of the Sun using solar oscillations, as we know it today began when Duvall and Harvey (1983) determined frequencies of a reasonably large number of solar oscillation modes covering a wide range of horizontal wavelengths. Since then, many more sets of solar oscillation frequencies have been published. A large fraction of the early work in the field was based on the frequencies determined by Libbrecht et al. (1990) from observations made at the Big Bear Solar Observatory (BBSO).

Accurate and precise estimates of solar frequencies require long, uninterrupted observations of the Sun, that are possible only with a network of telescopes. The Birmingham Solar Oscillation Network (BiSON; Elsworth et al. 1991; Chaplin et al. 2007) and the International Research on the Interior of the Sun (IRIS; Fossat 1991) were two of the first networks. Both these networks however, did Sun-as-a-star (i.e., one pixel) observations, as a result they could only observe the low-degree modes with $\ell$ of $0-3$. To overcome this limitation, resolved-disc measurements were needed. This resulted in the construction of the Global Oscillation Network Group (GONG: Hill et al. 1996). This ground-based network has been making full-disc observations of the Sun since 1995. There were concurrent developments in space based observations and the Solar and Heliospheric Observatory (SoHO: Domingo et al. 1995) was launched in 1995. Among SoHO's observing programme were three helioseismology related ones, the 'Solar Oscillations Investigation' (SOI) using the Michelson Doppler Imager(MDI: Scherrer et al. 1995), 'Variability of solar Irradiance and Gravity Oscillations' (VIRGO: Lazrek et al. 1997) and 'Global Oscillations at Low Frequencies' (GOLF: Gabriel et al. 1997). Of these, MDI was capable of observing intermediate and high degree modes. BiSON and GONG continue to observe the Sun from the 
ground; MDI stopped acquiring data in April 2011. MDI has been succeeded by the Heliospheric and Magnetic Imager (HMI: Schou et al. 2012; Scherrer et al. 2012) on board the Solar Dynamics Observatory (SDO: Pesnell et al. 2012). In addition to obtaining better data, there have also been improvements in techniques to determine mode frequencies from the data (e.g., Larson and Schou 2008; Korzennik et al. 2013a) which has facilitated detailed helioseismic analyses. Descriptions of some of the early developments in the field can be found in the proceedings of the workshop "Fifty Years of Seismology of the Sun and Stars" (Jain et al. 2013).

Asteroseismology, the seismic study of stars other than the Sun, took longer to develop because of inherent difficulties in ground-based observations. Early attempts were focused on trying to observe pulsations of $\alpha$ Cen. Some early ground-based attempts did not find any convincing evidence for solar-like pulsations (e.g., Brown and Gilliland 1990), though others could place limits (e.g., Pottasch et al. 1992; Edmonds and Cram 1995). It took many more attempts before pulsations of $\alpha$ Cen were observed and the frequencies measured (Bouchy and Carrier 2001; Bedding et al. 2004; Kjeldsen et al. 2005). Other stars were targeted too (e.g., Kjeldsen et al. 2003; Carrier et al. 2005a, b; Bedding et al. 2007; Arentoft et al. 2008). The field did not grow till spacebased missions were available. The story of space-based asteroseismology started with the ill-fated Wide-Field Infrared Explorer (WIRE). The satellite failed because coolants meant to keep the detector cool evaporated, but Buzasi (2000) realised that the star tracker could be used to monitor stellar variability and hence to look for stellar oscillations. This followed the observations of $\alpha \mathrm{UMa}$ and $\alpha \mathrm{Cen} \mathrm{A}$ (Buzasi et al. 2000; Schou and Buzasi 2001; Fletcher et al. 2006). The Canadian mission Microvariability and Oscillations of Stars (MOST: Walker et al. 2003) was the first successfully launched mission dedicated to asteroseismic studies. Although it was not very successful in studying solar type stars, it was immensely successful in studying giants, classical pulsators, and even star spots and exo-planets. The next major step was the ESA/French CoRoT mission (Baglin et al. 2006; Auvergne et al. 2009). CoRot observed many giants and showed giants also show non-radial pulsations (De Ridder et al. 2009); the mission observed some subgiants and main sequence stars as well (see e.g., Deheuvels et al. 2010; Ballot et al. 2011; Deheuvels and the CoRoT Team 2014). While CoRoT and MOST showed that the seismic study of other stars was feasible, the field began to flourish after the launch of the Kepler mission (Koch et al. 2010) and the demonstration that Kepler could indeed observe stellar oscillations (Gilliland et al. 2010) and that the data could be used to derive stellar properties (Chaplin et al. 2010). Asteroseismology is going through a phase in which we are still learning the best ways to analyse and interpret the data, but with two more asteroseismology missions being planned - the Transiting Exoplanet Survey Satellite (TESS; launch 2017), and PLATO (launch 2025) — this field is going to grow even more rapidly. We discuss the basics of asteroseismology in Sect. 11 of this review, however, only a dedicated review can do proper justice to the field.

This review is organised as follows: Since it is not possible to perform helioseismic (or for that matter asteroseismic) analyses without models, we start with the construction of solar and stellar models in Sect. 2. We then proceed to derive the equations of stellar oscillation and describe some the properties of the oscillations in Sect. 3. A brief history of solar models is given in Sect. 4. We show what happens when 
we try to compare solar models with the Sun by comparing frequencies in Sect. 5 . The difficulty in making such comparisons leads us to Sect. 6 where we show how solar oscillation frequencies may be inverted to infer properties of the solar interior. The next three sections are devoted to results. In Sect. 7 we describe what we have learnt about spherically symmetric part of solar structure. Deviations from spherical symmetry - in terms of dynamics, magnetic fields and structural asymmetries-are described in Sect. 8. The solar-cycle related changes in solar frequencies and the deduced changes in the solar interior are discussed in Sect. 9. Most of the results discussed in this review were obtained by analysing the frequencies of solar oscillation. There are other observables though, such as line-width and amplitude, and these carry information on how modes are excited (and damped). The issue of mode-excitation is discussed briefly in Sect. 10. Finally, in Sect. 11, we give a brief introduction to the field of asteroseismology.

\section{Modelling stars}

In order to put results obtained from seismic analyses in a proper context, we first give a short overview of the process of constructing models and of the inputs used to construct them. Traditionally in astronomy, we make inferences by comparing properties of models with data, which in the seismic context means comparing the computed frequencies with the observed ones. Thus, seismic investigations of the Sun and other stars start with the construction models and the calculation of their oscillation frequencies. In this section we briefly cover the field of modelling. We also discuss how solar models are constructed in a manner that is different from the construction of models of other stars. There are many excellent textbooks that describe stellar structure and evolution and hence, we only describe the basic equations and inputs. Readers are referred to books such as Kippenhahn et al. (2012), Huang and Yu (1998), Hansen et al. (2004), Weiss et al. (2004), Maeder (2009), etc. for details.

\subsection{The equations}

The most common assumption involved in making solar and stellar models is that stars are spherically symmetric, i.e., stellar properties are only a function of radius. This is a good approximation for non-rotating and slowly-rotating stars. The other assumption that is usually made is that a star does not change its mass as it evolves; this assumption is valid except for the very early stages of star formation and very evolved stages, such at the tip of the red giant branch. The Sun for instance, loses about $10^{-14}$ of its mass per year. Thus, in its expected main-sequence lifetime of about $10 \mathrm{Gyr}$, the Sun will lose only about $0.01 \%$ of its mass. The radius of a star on the other hand, is expected to change significantly. As a result, mass is used as the independent variable when casting the equations governing stellar structure and evolution.

The first equation is basically the continuity equation in the absence of flows, and is thus a statement of the conservation of mass: 


$$
\frac{\mathrm{d} r}{\mathrm{~d} m}=\frac{1}{4 \pi r^{2} \rho},
$$

where $m$ is the mass enclosed in radius $r$ and $\rho$ the density.

The next equation is a statement of the conservation of momentum in the quasistationary state. In the stellar context, it represents hydrostatic equilibrium:

$$
\frac{\mathrm{d} P}{\mathrm{~d} m}=-\frac{G m}{4 \pi r^{4}}
$$

Conservation of energy comes next. Stars produce energy in the core. At equilibrium, energy $l$ flows through a shell of radius $r$ per unit time as a result of nuclear reactions in the interior. If $\epsilon$ be the energy released per unit mass per second by nuclear reactions, and $\epsilon_{v}$ the energy lost by the star because of neutrinos streaming out without depositing their energy, then,

$$
\frac{\mathrm{d} l}{\mathrm{~d} m}=\epsilon-\epsilon_{\mathrm{v}}
$$

However, this is not enough. Different layers of a star can expand or contract during their evolution; for instance in the sub-giant and red-giant stages the stellar core contracts rapidly while the outer layers expand. Thus, Eq. (4) has to be modified to include the energy used or released as a result of expansion or contraction, and one can show that

$$
\frac{\mathrm{d} l}{\mathrm{~d} m}=\epsilon-\epsilon_{v}-C_{P} \frac{\mathrm{d} T}{\mathrm{~d} t}+\frac{\delta}{\rho} \frac{\mathrm{d} P}{\mathrm{~d} t}
$$

where $C_{P}$ is the specific heat at constant pressure, $t$ is time, and $\delta$ is given by the equation of state and defined as

$$
\delta=-\left(\frac{\partial \ln \rho}{\partial \ln T}\right)_{P, X_{i}},
$$

where $X_{i}$ denotes composition. The last two terms of Eq. (5) above are often lumped together and called $\epsilon_{g}$ ( $g$ for gravity) because they denote the release of gravitational energy.

The next equation determines the temperature at any point. In general terms, and with the help of Eq. (3), this equation can be written quite trivially as

$$
\frac{\mathrm{d} T}{\mathrm{~d} m}=-\frac{G m T}{4 \pi r^{4} P} \nabla
$$

where $\nabla$ is the dimensionless "temperature gradient" $\mathrm{d} \ln T / \mathrm{d} \ln P$. The difficulty lies in determining what $\nabla$ is, and this depends on whether energy is being transported by radiation or convection. We shall come back to the issue of $\nabla$ presently.

The last set of equations deals with chemical composition as a function of position and time. There are three ways that the chemical composition at any point of a star can change: (1) nuclear reactions, (2) the changes in the boundaries of convection zones, and (3) diffusion and gravitational settling (usually simply referred to as diffusion) of helium and heavy elements and other mixing processes as well. 
The change of abundance because of nuclear reactions can be written as

$$
\frac{\partial X_{i}}{\partial t}=\frac{m_{i}}{\rho}\left[\sum_{j} r_{j i}-\sum_{k} r_{i k}\right]
$$

where $m_{i}$ is the mass of the nucleus of each isotope $i, r_{j i}$ is the rate at which isotope $i$ is formed from isotope $j$, and $r_{i k}$ is the rate at which isotope $i$ is lost because it turns into a different isotope $k$. The rates $r_{i k}$ are external inputs to models.

Convection zones are chemically homogeneous — eddies of moving matter carry their composition with them and when they break-up, the material gets mixed with the surrounding. This happens on timescales that are very short compared to the time scale of a star's evolution. If a convection zone exists in the region between two spherical shells of masses $m_{1}$ and $m_{2}$, the average abundance of any species $i$ in the convection zone is:

$$
\bar{X}_{i}=\frac{1}{m_{2}-m_{1}} \int_{m_{1}}^{m_{2}} X_{i} \mathrm{~d} m .
$$

In the presence of convective overshoot, the limits of the integral in Eq. (9) have to be change to denote the edge of the overshooting region. The rate at which $\bar{X}_{i}$ changes will depend on nuclear reactions in the convection zone, as well as the rate at which the mass limits $m_{1}$ and $m_{2}$ change. One can therefore write

$$
\begin{aligned}
\frac{\partial \bar{X}_{i}}{\partial t} & =\frac{\partial}{\partial t}\left(\frac{1}{m_{2}-m_{1}} \int_{m_{1}}^{m_{2}} X_{i} \mathrm{~d} m\right) \\
& =\frac{1}{m_{2}-m_{1}}\left[\int_{m_{1}}^{m_{2}} \frac{\partial X_{i}}{\partial t} \mathrm{~d} m+\frac{\partial m_{2}}{\partial t}\left(X_{i, 2}-\bar{X}_{i}\right)-\frac{\partial m_{1}}{\partial t}\left(X_{i, 1}-\bar{X}_{i}\right)\right],
\end{aligned}
$$

where $X_{i, 1}$ and $X_{i, 2}$ is the mass fraction of element $i$ at $m_{1}$ and $m_{2}$ respectively.

The gravitational settling of helium and heavy elements can be described by the process of diffusion and the change in abundance can be found with the help of the diffusion equation:

$$
\frac{\partial X_{i}}{\partial t}=D \nabla^{2} X_{i},
$$

where $D$ is the diffusion coefficient, and $\nabla^{2}$ is the Laplacian operator. The diffusion coefficient hides the complexity of the process and includes, in addition to gravitational settling, diffusion due to composition and temperature gradients. All three processes are generally simply called 'diffusion'. Other mixing process, such as those induced by rotation, are often included the same way by modifying $D$ (e.g., Richard et al. 1996). $D$ depends on the isotope under consideration, however, it is not uncommon for stellar-evolution codes to treat helium separately, and use a single value for all heavier elements.

Equations (2), (3), (5), and (7) together with the equations relating to change in abundances, form the full set of equations that govern stellar structure and evolution. In most codes, Eqs. (2), (3), (5), and (7) are solved for a given $X_{i}$ at a given time $t$. Time is then advanced, Eqs. (8), (10), and (11) are solved to give new $X_{i}$, and Eqs. (2), 
(3), (5), and (7) are solved again. Thus, we have two independent variables, mass $m$ and time $t$, and we look for solutions in the interval $0 \leq m \leq M$ (stellar structure) and $t \geq t_{0}$ (stellar evolution).

Four boundary conditions are required to solve the stellar structure equations. Two (on radius and luminosity) can be applied quite trivially at the centre. The remaining conditions (on temperature and pressure) need to be applied at the surface. The boundary conditions at the surface are much more complex than the central boundary conditions and are usually determined with the aid of simple stellar-atmosphere models. As we shall see later, atmospheric models plays a large role in determining the frequencies of different modes of oscillation.

The initial conditions needed to start evolving a star depend on where we start the evolution. If the evolution begins at the pre-main sequence phase, i.e., while the star is still collapsing, the initial structure is quite simple. Temperatures are low enough to make the star fully convective and hence chemically homogeneous. If evolution is begun at the Zero Age Main Sequence (ZAMS), which is the point at which hydrogen fusion begins, a ZAMS model must be used.

We return to the question of the temperature gradient $\nabla$ in Eq. (7). If energy is transported by radiation ("the radiative zone"), $\nabla$ is calculated assuming that energy transport can be modelled as a diffusive process, and that yields

$$
\nabla=\nabla_{\mathrm{rad}}=\frac{3}{64 \pi \sigma G} \frac{\kappa l P}{m T^{4}}
$$

where, $\sigma$ is the Stefan-Boltzmann constant and $\kappa$ is the opacity which is an external input to stellar models.

The situation in the convection zones is tricky. Convection is, by its very nature, a three-dimensional phenomenon, yet our models are one dimensional. Even if we construct three-dimensional models, it is currently impossible include convection and evolve the models at the same time. This is because convection takes place over time scales of minutes to hours, while stars evolve in millions to billions of years. As a result, drastic simplifications are used to model convection. Deep inside a star, the temperature gradient is well approximated by the adiabatic temperature gradient $\nabla_{\mathrm{ad}} \equiv(\partial \ln T / \partial \ln P)_{s}$ ( $s$ being the specific entropy), which is determined by the equation of state. This approximation cannot be used in the outer layers where convection is not efficient and some of the energy is also carried by radiation. In these layers one has to use an approximate formalism since there is no "theory" of stellar convection as such. One of the most common formulations used to calculate convective flux in stellar models is the so-called "mixing length theory" (MLT). The mixing length theory was first proposed by Prandtl (1925). His model of convection was analogous to heat transfer by particles; the transporting particles are macroscopic eddies and their mean free path is the "mixing length". This was applied to stars by Biermann (1948), Vitense (1953), and Böhm-Vitense (1958). Different mixing length formalisms have slightly different assumptions about what the mixing length is. The main assumption in the usual mixing length formalism is that convective eddies move an average distance equal to the mixing length $l_{m}$ before giving up their energy and losing their identity. The mixing length is usually defined 
as $l_{m}=\alpha H_{P}$, where $\alpha$, a constant, is the so-called 'mixing length parameter', and $H_{P} \equiv-\mathrm{d} r / \mathrm{d} \ln P$ is the pressure scale height. Details of how $\nabla$ is calculated under these assumption can be found in any stellar structure textbook, such as Kippenhahn et al. (2012). There is no a priori way to determine $\alpha$, and it is one of the free parameters in stellar models. Variants of MLT are also used, and among these are the formulation of Canuto and Mazzitelli (1991) and Arnett et al. (2010).

\subsection{Inputs to stellar models}

The equations of stellar structure look quite simple. Most of the complexity is hidden in the external inputs needed to solve the equations. There are four important inputs that are needed: the equation of state, radiative opacities, nuclear reaction rates and coefficients to derive the rates of diffusion and gravitational settling. These are often referred to as the microphysics of stars.

\subsubsection{The equation of state}

The equation of state specifies the relationship between density, pressure, temperature and composition. The stellar structure equations are a set of five equations in six unknowns, $r, P, l, T, X_{i}$, and $\rho$. None of the equations directly solves for the behaviour of $\rho$ as a function of mass and time. We determine the density using the equation of state.

The ideal gas equation is good enough to make simple models. However, the ideal gas law does not apply to all layers of stars since it does not include the effects of ionisation, radiation pressure, pressure ionisation, degeneracy, etc. Among the early published equations of state valid under stellar condition is that of Eggleton, Faulkner and Flannery (EFF; Eggleton et al. 1973). This equation of state suffered from the fact that it did not include corrections to pressure due to Coulomb interactions, and this led to the development of the so-called "Coulomb Corrected" EFF, or CEFF equation of state (Christensen-Dalsgaard and Däppen 1992; Guenther et al. 1992). However, the CEFF equation of state is not fully thermodynamically consistent.

Modern equations of state are usually given in a tabular form with important thermodynamic quantities such as $\nabla_{\mathrm{ad}}, C_{p}$ listed as a function of $T, P$ (or $\rho$ ) and composition. These include the OPAL equation of state (Rogers et al. 1996; Rogers and Nayfonov 2002) and the so-called MHD (i.e., Mihalas, Hummer and Däppen) equation of state (Däppen et al. 1988; Mihalas et al. 1988; Hummer and Mihalas 1988; Gong et al. 2001). Both OPAL and MHD equations of state suffer from the limitation that unlike the EFF and CEFF equations of state, the heavy-element mixture used to calculate them cannot be changed by the user. This has lead to the development of thermodynamically consistent extensions of the EFF equation of state that allow users to change the equation of state easily. The SIREFF equation of state is an example of this (Guzik and Swenson 1997; Guzik et al. 2005). 


\subsubsection{Opacities}

We need to know the opacity $\kappa$ of the stellar material in order to calculate $\nabla_{\text {rad }}$ [Eq. (12)]. Opacity is a measure of how opaque a material is to photons. Like modern equations of state, opacities are usually available in tabular form as a function of density, temperature and composition. Among the widely used opacity tables are the OPAL (Iglesias and Rogers 1996) and OP (Badnell et al. 2005; Mendoza et al. 2007) tables. The OPAL opacity tables include contributions from 19 heavy elements whose relative abundances (by numbers) with respected to hydrogen are larger than about $10^{-7}$. The OP opacity calculations include 15 elements. Neither table is very good at low temperature where molecules become important. As a results these tables are usually supplemented by specialised low-temperature opacity tables such that those of Kurucz (1991) and Ferguson et al. (2005).

\subsubsection{Nuclear reaction rates}

Nuclear reaction rates are required to compute energy generation, neutrino fluxes and composition changes. The major sources of reaction rates for solar models are the compilations of Adelberger et al. (1998, 2011) and Angulo et al. (1999).

\subsubsection{Diffusion coefficients}

The commonly used prescriptions for calculating diffusion coefficients are those of Thoul et al. (1994) and Proffitt and Michaud (1991).

\subsubsection{Atmospheres}

While not a microphysics input, stellar atmospheric models are equally crucial: stellar models do not stop at $r=R$, but generally extend into an atmosphere, and hence the need for these models. These models are also used to calculate the outer boundary condition. The atmospheric models are often quite simple and provide a $T-\tau$ relation, i.e., a relation between temperature $T$ and optical depth $\tau$. The Eddington $T-\tau$ relation is quite popular. Semi-empirical relations such as the Krishna Swamy $T-\tau$ relation (Krishna Swamy 1966), the Vernazza, Avrett and Loeser (VAL) relation (Vernazza et al. 1981) though applicable to the Sun are also used frequently to model other stars. A relatively recent development in the field is the use of $T-\tau$ relations obtained from simulations of convection in the outer layers of stars.

\subsection{The concept of "standard" solar models}

The mass of a star is the most fundamental quantity needed to model a star. Other input quantities include the initial heavy-element abundance $Z_{0}$ and the initial helium abundance $Y_{0}$. These quantities affect both the structure and the evolution of star through their influence on the equation of state and opacities. Also required is the mixing-length parameter $\alpha$. Once these quantities are known, or chosen, models are 
Table 1 Global parameters of the Sun

\begin{tabular}{lll}
\hline Quantity & Estimate & Reference \\
\hline Mass $\left(M_{\odot}\right)^{\mathrm{a}}$ & $1.98892(1 \pm 0.00013) \times 10^{33} \mathrm{~g}$ & Cohen and Taylor (1987) \\
Radius $\left(R_{\odot}\right)^{\mathrm{b}}$ & $6.9599(1 \pm 0.0001) \times 10^{10} \mathrm{~cm}$ & Allen (1973) \\
Luminosity $\left(L_{\odot}\right)$ & $3.8418(1 \pm 0.004) \times 10^{33} \mathrm{ergs} \mathrm{s}^{-1}$ & Fröhlich and Lean (1998) \\
Age & $4.57(1 \pm 0.0044) \times 10^{9}$ years & Bahcall et al. (1995) \\
\hline
\end{tabular}

${ }^{a}$ Derived from the values of $G$ and $G M_{\odot}$

${ }^{\mathrm{b}}$ See Schou et al. (1997), Antia (1998), Brown and Christensen-Dalsgaard (1998) and Haberreiter et al. (2008) for more recent discussions about the exact value of the solar radius

Table 2 Nominal solar conversion constants as per IAU resolution $\mathrm{B} 3$

See Mamajek et al. (2015) for details

\begin{tabular}{ll}
\hline Parameter & value \\
\hline $1 \mathcal{R}_{\odot}^{\mathrm{N}}$ & $6.957 \times 10^{8} \mathrm{~m}$ \\
$1 \mathcal{S}_{\odot}^{\mathrm{N}}$ & $1361 \mathrm{~W} \mathrm{~m}^{-2}$ \\
$1 \mathcal{L}_{\odot}^{\mathrm{N}}$ & $3.828 \times 10^{26} \mathrm{~W}$ \\
$1 \mathcal{T}_{\mathrm{eff} \odot}^{\mathrm{N}}$ & $5772 \mathrm{~K}$ \\
$1 \mathcal{G} \mathcal{M}_{\odot}^{\mathrm{N}}$ & $1.3271244 \times 10^{20} \mathrm{~m}^{3} \mathrm{~s}^{-2}$ \\
\hline
\end{tabular}

evolved in time, until they reach the observed temperature and luminosity. The initial guess of the mass may not result in a models with the required characteristics, and a different mass needs to be chosen and the process repeated. Once a model that satisfies observational constraints is constructed, the model becomes a proxy for the star; the age of the star is assumed to be the age of the model, and the radius of the star is assumed to be the radius of the model. The most important source of uncertainty in stellar models is our inability to model convection properly. MLT requires a free parameter $\alpha$ that is essentially unconstrained and introduces uncertainties in the radius of a star of a given mass and heavy-element abundance. And even if we were able to constrain $\alpha$, it would not account for all the properties of convective heat transport and thus would therefore, introduce errors in the results.

The Sun is modelled in a somewhat different manner since its global properties are known reasonably well. We have independent estimates of the solar mass, radius, age and luminosity. The commonly used values are listed in Table 1. Solar properties are usually used a references to express the properties of other stars. Small differences in solar parameters adopted by different stellar codes can therefore, lead to differences. To mitigate this problem, the International Astronomical Union adopted a set of nominal values for the global properties of the Sun. These are listed in Table 2. Note that the symbols used to denote the nominal properties are different from the usual solar notation.

To be called a solar model, a $1 M_{\odot}$ model must have a luminosity of $1 L_{\odot}$ and a radius of $1 R_{\odot}$ at the solar age of $4.57 \mathrm{Gyr}$. The way this is done is by recognising that there are two constraints that we need to satisfy at $4.57 \mathrm{Gyr}$, the solar radius and 
luminosity, and that the set of equations has two free parameters, the initial helium abundance $Y_{0}$ and the mixing length parameter $\alpha$. Thus, we basically have a situation of two constraints and two unknowns. However, since the equations are non-linear, we need an iterative method to determine $\alpha$ and $Y_{0}$. The value of $\alpha$ obtained in this manner for the Sun is often called the "solar calibrated" value of $\alpha$ and used to model other stars. In addition to $\alpha$ and $Y_{0}$, and very often initial $Z$ is adjusted to get the observed $Z / X$ in the solar envelope. The solar model constructed in this manner does not have any free parameters, since these are determined to match solar constraints. Such a model is known as a "standard" solar model (SSM).

The concept of standard solar models is very important in solar physics. Standard solar models are those where only standard input physics such as equations of state, opacity, nuclear reaction rates, diffusion coefficients etc., are used. The parameters $\alpha$ and $Y_{0}$ (and sometime $Z_{0}$ ) are adjusted to match the current solar radius and luminosity (and surface $Z / X$ ). No other input is adjusted to get a better agreement with the Sun. By comparing standard solar models constructed with different input physics with the Sun we can put constraints on the input physics. One can use helioseismology to test whether or not the structure of the model agrees with that of the Sun.

There are many physical processes that are not included in SSMs. These are processes that do not have a generally recognised way of modelling and rely on free parameters. These additional free parameters would make any solar model that includes these processes "non standard". Among the missing processes are effects of rotation on structure and of mixing induced by rotation. There are other proposed mechanisms for mixing in the radiative layers of the Sun, such as mixing caused by waves generated at the convection-zone base (e.g., Kumar et al. 1999) that are not included either. These missing processes can affect the structure of a model. For example, Turck-Chièze et al. (2004) found that mixing below the convection-zone base can change both the position of the convection-zone base (gets shallower) and the helium abundance (abundance increases). However, their inclusion in stellar models require us to choose values for the parameters in the formulaæ that describe the processes. Accretion and mass-loss at some stage of solar evolution can also affect the solar models (Castro et al. 2007), but again, there are no standard formulations for modelling these effects.

Standard solar models constructed by different groups are not identical. They depend on the microphysics used, as well as assumption about the heavy element abundance. Numerical schemes also play a role. For a discussion of the sources of uncertainties in solar models, readers are referred to Section 2.4 of Basu and Antia (2008).

\section{The equations governing stellar oscillations}

To a good approximation, solar oscillations and solar-like oscillations in other stars, can be described as linear and adiabatic. In the case of the Sun, modes of oscillation have amplitudes of the order of $10 \mathrm{~cm} \mathrm{~s}^{-1}$ while the sound speed at the surface is more like $10 \mathrm{~km} \mathrm{~s}^{-1}$, putting the amplitudes squarely in the linear regime. The condition of adiabaticity can be justified over most of the Sun-the oscillation frequencies have periods of order $5 \mathrm{~min}$, but the thermal time-scale is much larger and hence, adiabaticity 
applies. This condition however breaks down in the near-surface layers (where thermal time-scales are short) resulting in an error in the frequencies. This error adds to what is later described as the "surface term" (see Sect. 5) but which can be filtered out in many cases. We shall retain the approximations of linearity and adiabaticity in this section since most helioseismic results have been obtained under these assumptions after applying a few corrections.

Details of the derivation of the oscillation equations and a description of their properties has been described well by authors such as Cox (1980), Unno et al. (1989), Christensen-Dalsgaard and Berthomieu (1991), Gough (1993), etc. Here we give a short overview that allows us to derive some of the properties of solar and stellar oscillations that allow us to undertake seismic studies of the Sun and other stars.

The derivation of the equations of stellar oscillations begins with the basic equations of fluid dynamics, i.e., the continuity equation and the momentum equation. We use the Poisson equation to describe the gravitational field. Thus, the basic equations are:

$$
\begin{aligned}
& \frac{\partial \rho}{\partial t}+\nabla \cdot(\rho \mathbf{v})=0 \\
& \rho\left(\frac{\partial v}{\partial t}+\mathbf{v} \cdot \nabla \mathbf{v}\right)=-\nabla P+\rho \nabla \Phi
\end{aligned}
$$

and

$$
\nabla^{2} \Phi=4 \pi G \rho,
$$

where $\mathbf{v}$ is the velocity of the fluid element, $\Phi$ is the gravitational potential, and $G$ the gravitational constant. The heat equation is written in the form

$$
\frac{\mathrm{d} q}{\mathrm{~d} t}=\frac{1}{\rho\left(\Gamma_{3}-1\right)}\left(\frac{\mathrm{d} p}{\mathrm{~d} t}-\frac{\Gamma_{1} P}{\rho} \frac{\mathrm{d} \rho}{\mathrm{d} t}\right),
$$

where

$$
\Gamma_{1}=\left(\frac{\partial \ln P}{\partial \ln \rho}\right)_{\mathrm{ad}}, \quad \text { and, } \quad \Gamma_{3}-1=\left(\frac{\partial \ln T}{\partial \ln \rho}\right)_{\mathrm{ad}} .
$$

In the adiabatic limit Eq. (16) reduces to

$$
\frac{\partial P}{\partial t}+\mathbf{v} \cdot \nabla P=c^{2}\left(\frac{\partial \rho}{\partial t}+\mathbf{v} \cdot \nabla \rho\right)
$$

where $c=\sqrt{\Gamma_{1} P / \rho}$ is the sound speed

Linear oscillation equations are a result of linear perturbations to the fluid equations above. Thus, e.g., we can write the perturbations to density as

$$
\rho(\mathbf{r}, t)=\rho_{0}(\mathbf{r})+\rho_{1}(\mathbf{r}, t)
$$

where the subscript 0 denotes the equilibrium, spherically symmetric, quantity which by definition does not depend on time, and the subscript 1 denotes the perturbation. Perturbations to other quantities can be written in the same way. Note that Eq. (19) 
shows the Eulerian perturbation to density, i.e., perturbations at a fixed point in space denoted by co-ordinates $(r, \theta, \phi)$. In some cases, notably when there are time derivatives, it is easier to use the Lagrangian perturbation, i.e., a perturbation seen by an observer moving with the fluid. The Lagrangian perturbation for density is given by

$$
\delta \rho(r, t)=\rho(\mathbf{r}+\boldsymbol{\xi}(\mathbf{r}, t))-\rho(\mathbf{r})=\rho_{1}(\mathbf{r}, t)+\boldsymbol{\xi}(\mathbf{r}, t) \cdot \nabla \rho,
$$

where $\xi$ is the displacement from the equilibrium position. The perturbations to the other quantities can be written in the same way. The equilibrium state of a star is generally assumed to be static, and thus the velocity $\mathbf{v}$ in the fluid equations appears only after a perturbation has been applied and is nothing but the rate of change of displacement of the fluid, i.e., $\mathbf{v}=\mathrm{d} \xi / \mathrm{d} t$.

Substituting the perturbed quantities in Eqs. (13), (14) and (15), and keeping only linear terms in the perturbation, we get

$$
\begin{aligned}
& \rho_{1}+\nabla \cdot\left(\rho_{0} \xi\right)=0, \\
& \rho \frac{\partial^{2} \xi}{\partial t^{2}}=-\nabla P_{1}+\rho_{0} \nabla \Phi_{1}+\rho_{1} \nabla \Phi_{0}, \\
& \nabla^{2} \Phi_{1}=4 \pi G \rho_{1} .
\end{aligned}
$$

It is easier to consider the Lagrangian perturbation for the heat equation since under the assumptions that at have been made, the time derivative of the various quantities is simply the time derivative of the Lagrangian perturbation of those quantities. Thus, from Eq. (16) we get

$$
\frac{\partial \delta q}{\partial t}=\frac{1}{\rho_{0}\left(\Gamma_{3.0}-1\right)}\left(\frac{\partial \delta P}{\partial t}-\frac{\Gamma_{1,0} P_{0}}{\rho_{0}} \frac{\partial \delta \rho}{\partial t}\right) .
$$

In the adiabatic limit, where energy loss is negligible, $\partial \delta q / \partial t=0$ and

$$
P_{1}+\boldsymbol{\xi} \cdot \nabla P=\frac{\Gamma_{1,0} P_{0}}{\rho_{0}}\left(\rho_{1}+\boldsymbol{\xi} \cdot \nabla \rho\right) .
$$

The term $\Gamma_{1,0} P_{0} / \rho_{0}$ in Eq. (25) is nothing but the squared, unperturbed sound speed $c_{0}^{2}$.

In the subsequent discussion, we drop the subscript ' 0 ' for the equilibrium quantities, and only keep the subscript for the perturbations.

\subsection{The spherically symmetric case}

Since stars are usually spherical, though not necessarily spherically symmetric, it is customary to write the equations in spherical-polar coordinates with the origin define at the centre of the star, with $r$ being the radial distance, $\theta$ the co-latitude, and $\phi$ the longitude. The different quantities can be decomposed into their radial and tangential components. Thus, the displacement $\xi$ can be decomposed as 


$$
\xi=\xi_{r} \hat{a}_{r}+\xi_{t} \hat{a}_{t}
$$

where, $\hat{a}_{r}$ and $\hat{a}_{t}$ are the unit vectors in the radial and tangential directions respectively, $\xi_{r}$ is the radial component of the displacement vector and $\xi_{t}$ the transverse component. An advantage of using spherical polar coordinates is the fact that tangential gradients of the equilibrium quantities do not exist. Thus, e.g., the heat equation [Eq. (25)] becomes

$$
\rho_{1}=\frac{\rho}{\Gamma_{1} P} P_{1}+\rho \xi_{r}\left(\frac{1}{\Gamma_{1} P} \frac{\mathrm{d} P}{\mathrm{~d} r}-\frac{1}{\rho} \frac{\mathrm{d} \rho}{\mathrm{d} r}\right) .
$$

The tangential component of the equation of motion [Eq. (14)] is

$$
\rho \frac{\partial^{2} \xi_{\mathbf{t}}}{\partial t^{2}}=-\nabla_{t} P_{1}+\rho \nabla_{t} \Phi^{\prime}
$$

or (taking the tangential divergence of both sides),

$$
\rho \frac{\partial^{2}}{\partial t^{2}}\left(\nabla_{t} \cdot \boldsymbol{\xi}_{\mathbf{t}}\right)=-\nabla_{t}^{2} P_{1}+\rho \nabla_{t}^{2} \Phi_{1} .
$$

The continuity equation [Eq. (21)] after decomposition can be used to eliminate the term $\nabla_{t} \cdot \xi_{\mathbf{t}}$ from Eq. (29) to obtain

$$
-\frac{\partial^{2}}{\partial t^{2}}\left[\rho^{\prime}+\frac{1}{r^{2}} \frac{\partial}{\partial r}\left(\rho r^{2} \xi_{r}\right)\right]=-\nabla_{t}^{2} P_{1}+\rho \nabla_{t}^{2} \Phi_{1} .
$$

The radial component of the equation of motion gives

$$
\rho \frac{\partial^{2} \xi_{r}}{\partial t^{2}}=-\frac{\partial P_{1}}{\partial r}-\rho_{1} g+\rho \frac{\partial \Phi_{1}}{\partial r},
$$

where we have used the fact that gravity acts in the negative $r$ direction. Finally, the Poisson's equation becomes

$$
\frac{1}{r^{2}} \frac{\partial}{\partial r}\left(r^{2} \frac{\partial \Phi_{1}}{\partial r}\right)+\nabla_{t}^{2} \Phi_{1}=-4 \pi G \rho_{1} .
$$

Note that the there are no mixed radial and tangential derivatives, and the tangential gradients appear only as the tangential component of the Laplacian, and as a result one can show that (e.g., Christensen-Dalsgaard 2003) the tangential part of the perturbed quantities can be written in terms of eigenfunctions of the tangential Laplacian operator. Since we are dealing with spherical objects, the spherical harmonic function are used.

Furthermore, note that time $t$ does not appear explicitly in coefficients of any of the derivatives. This implies that the time dependent part can be separated out from the spatial part, and the time-dependence can be expressed in terms of $\exp (-i \omega t)$ where 
$\omega$ can be real (giving an oscillatory solution in time) or imaginary (a solution that grows or decays). Thus, we may write:

$$
\begin{aligned}
& \xi_{r}(r, \theta, \phi, t) \equiv \xi_{r}(r) Y_{\ell}^{m}(\theta, \phi) \exp (-i \omega t), \\
& P_{1}(r, \theta, \phi, t) \equiv P_{1}(r) Y_{\ell}^{m}(\theta, \phi) \exp (-i \omega t),
\end{aligned}
$$

etc.

Once we have the description of the variables in terms of an oscillating function of time, and spherical harmonic functions in the angular directions, we can substitute those in Eqs. (30), (31) and (32). Furthermore, Eq. (27) can be used to eliminate the quantity $\rho_{1}$ to obtain

$$
\frac{\mathrm{d} \xi_{r}}{\mathrm{~d} r}=-\left(\frac{2}{r}+\frac{1}{\Gamma_{1} P} \frac{\mathrm{d} P}{\mathrm{~d} r}\right) \xi_{r}+\frac{1}{\rho c^{2}}\left(\frac{S_{\ell}^{2}}{\omega^{2}}-1\right) P_{1}-\frac{\ell(\ell+1)}{\omega^{2} r^{2}} \Phi_{1},
$$

where $c^{2}=\Gamma_{1} P / \rho$ is the squared sound speed, and $S_{\ell}^{2}$ is the Lamb frequency defined by

$$
S_{\ell}^{2}=\frac{\ell(\ell+1) c^{2}}{r^{2}}
$$

Equation (31) and the equation of hydrostatic equilibrium give

$$
\frac{\mathrm{d} P_{1}}{\mathrm{~d} r}=\rho\left(\omega^{2}-N^{2}\right) \xi_{r}+\frac{1}{\Gamma_{1} P} \frac{\mathrm{d} P}{\mathrm{~d} r} P_{1}+\rho \frac{\mathrm{d} \Phi_{1}}{\mathrm{~d} r},
$$

where, $N$ is Brunt-Väisälä or buoyancy frequency defined as

$$
N^{2}=g\left(\frac{1}{\Gamma_{1} P} \frac{\mathrm{d} P}{\mathrm{~d} r}-\frac{1}{\rho} \frac{\mathrm{d} \rho}{\mathrm{d} r}\right) .
$$

This is the frequency with which a small element of fluid will oscillate when it is disturbed from its equilibrium position. When $N^{2}<0$ the fluid is unstable to convection and in such regions part of the energy will be transported by convection. Finally, Eq. (32) becomes

$$
\frac{1}{r^{2}} \frac{\mathrm{d}}{\mathrm{d} r}\left(r^{2} \frac{\mathrm{d} \Phi_{1}}{\mathrm{~d} r}\right)=-4 \pi G\left(\frac{P_{1}}{c^{2}}+\frac{\rho \xi_{r}}{g} N^{2}\right)+\frac{\ell(\ell+1)}{r^{2}} \Phi_{1} .
$$

Equations (35), (37) and (39) form a set of fourth-order differential equations and constitute an eigenvalue problem with eigenfunction $\omega$. Solution of the equations yields the radial component, $\xi_{r}$ of the displacement eigenfunction as well as $P_{1}, \Phi_{1}$ as well as $\mathrm{d} \Phi_{1} / \mathrm{d} r$. Each eigenvalue is usually referred to as a "mode" of oscillation.

The transverse component of displacement vector can be written in terms of $P_{1}(r)$ and $\Phi_{1}(r)$ and one can show that

$$
\boldsymbol{\xi}_{\mathbf{t}}(r, \theta, \phi, t)=\xi_{t}(r)\left(\frac{\partial Y_{\ell}^{m}}{\partial \theta} \hat{a_{\theta}}+\frac{1}{\sin \theta} \frac{\partial Y_{\ell}^{m}}{\partial \phi} \hat{a_{\phi}}\right) \exp (-i \omega t),
$$


where $\hat{a_{\theta}}$ and $\hat{a_{\phi}}$ are the unit vectors in the $\theta$ and $\phi$ directions respectively, and

$$
\xi_{t}(r)=\frac{1}{r \omega^{2}}\left(\frac{1}{\rho} P_{1}(r)-\Phi_{1}(r)\right)
$$

Note that there is no $n$ or $m$ dependence in the Eqs. (35)-(41). The different eigenvalues for a given value of $\ell$ are given the label $n$. Conventionally $n$ can be any signed integer and can be positive, zero or negative depending on the type of the mode. In general $|n|$ represents the number of nodes the radial eigenfunction has in the radial direction. As mentioned in the Introduction, values of $n>0$ are used to specify acoustic modes, $n<0$ label gravity models and $n=0$ labels $\mathrm{f}$ modes. Of course, this labelling gets more complicated when mixed modes (see Sect. 11) are present, but this works well for the Sun. It is usual to denote the eigenfunctions with $n$ and $\ell$, thus, for example, the total displacement is denoted as $\xi_{n, \ell}$ and the radial and transverse components as $\xi_{r, n, \ell}$ and $\xi_{t, n, \ell}$ respectively. The lack of the $m$ dependence in the equations has to do with the fact that we are considering a spherically symmetric system, and to define $m$ one needs to break the symmetry. Thus, in a spherically symmetric system, all modes are of a given value of $n$ and $\ell$ are degenerate in $m$. Rotation, magnetic fields and other asphericities lift this degeneracy.

An important property of all modes is their mode inertia defined as

$$
E_{n, \ell}=\int_{V} \rho \boldsymbol{\xi}_{n, \ell} \cdot \boldsymbol{\xi}_{n, \ell} \mathrm{d}^{3} \mathbf{r}=\int_{0}^{R} \rho\left[\xi_{r, n, \ell}^{2}+\ell(\ell+1) \xi_{t, n, \ell}^{2}\right] r^{2} \mathrm{~d} r
$$

(Christensen-Dalsgaard 2003). Modes that penetrate deeper into the star (which as we shall see later are low degree modes) have higher mode inertia than those that do not penetrate as deep (higher degree modes). Additionally, for modes of a given degree, higher frequency modes have larger inertia than lower frequency modes. For a given perturbation, frequencies of low-inertia modes affected by the perturbation change more than those of affected high-inertia modes. Since the normalisation of eigenfunctions can be arbitrary, it is conventional to normalise $E_{n, \ell}$ explicitly as

$$
E_{n, \ell}=\frac{\int_{0}^{R} \rho\left[\left|\xi_{r, n, \ell}\right|^{2}+\ell(\ell+1)\left|\xi_{t, n, \ell}\right|^{2}\right] r^{2} \mathrm{~d} r}{M\left[\left|\xi_{r, n, \ell}(R)\right|^{2}+\ell(\ell+1)\left|\xi_{t, n, \ell}(R)\right|^{2}\right]}
$$

where, $M$ is the total mass and $R$ is the total radius.

\subsection{Boundary conditions}

The discussion of the equations of stellar evolution cannot be completed without discussing the boundary conditions that are need to actually solve the equations. For details of the boundary conditions and how they can be obtained, the reader is referred to Cox (1980) and Unno et al. (1989). We give a brief overview.

A complete solution of the equations requires four boundary conditions. An inspection of the equations shows that they have a singular point at $r=0$, however, it is a 
singularity that allows both regular and singular solutions. Given that we are talking of physical systems, we need to look for solutions that are regular at $r=0$. As is usual, the central boundary conditions are obtained by expanding the solution around $r=0$. This reveals that as $r \rightarrow 0, \xi_{r} \propto r$ for $\ell=0$ modes, and $\xi_{r} \propto r^{\ell-1}$ for others. The quantities $P_{1}$ and $\Phi_{1}$ vary as $r^{\ell}$.

The surface boundary conditions are complicated by the fact that the "surface" of a star is not well defined in terms of density, pressure etc., but is determined by the way the stellar atmosphere is treated. In fact once can show that the calculated frequencies can change substantially depending on where the one assumes the outer boundary of the star is (see e.g., Hekker et al. 2013). The usual assumption that is made is that $\Phi_{1}$ and $\mathrm{d} \Phi_{1} / \mathrm{d} r$ are continuous at the surface. Under the simple assumption that $\rho_{1}$ is zero at the surface, the Poisson equation can be solved to show that for $\Phi_{1}$ to be zero at infinity,

$$
\Phi_{1}=A r^{-1-\ell},
$$

where $A$ is a constant. It follow that

$$
\frac{\mathrm{d} \Phi_{1}}{\mathrm{~d} r}+\frac{\ell+1}{r} \Phi_{1}=0
$$

at the surface.

Another condition can be applied by assuming that the pressure on the deformed surface of the star be zero, in other words that the Lagrangian perturbation of pressure be zero at the surface, i.e.,

$$
\delta P=P_{1}+\xi_{r} \frac{\mathrm{d} P}{\mathrm{~d} r}=0
$$

at the surface. This condition implies that $\nabla \cdot \xi \sim 0$ at the outer boundary. Combining this with Eq. (35) we get $\xi_{r}=P_{1} / g \rho$ at the surface. If $\Phi_{1}$ is neglected, i.e., the so-called Cowling approximation is used, this reduces to

$$
\xi_{r}=\xi_{t} \omega^{2} .
$$

This is worth noting that the equations of stellar oscillation are generally solved after expressing all the physical quantities in terms of dimensionless numbers. The dimensionless frequency $\sigma$ is expressed as

$$
\sigma^{2}=\frac{R^{3}}{G M} \omega^{2}
$$

where, $R$ is the radius of the star and $M$ the mass. Similarly the dimensionless radius $\hat{r}=r / R$, dimensionless pressure is given by $\widehat{P}=\left(R^{4} / G M^{2}\right) P$ and the dimensionless density by $\hat{\rho}=\left(R^{3} / M\right) \rho$. These relations lead us to the dimensionless expressions for the perturbed quantities. It can be shown very easily that $\hat{\xi}_{r}$ the dimensionless displacement eigenfunction, $\widehat{P}_{1}$ the dimensionless pressure perturbation, and $\widehat{\Phi}_{1}$ the dimensionless perturbation to the gravitational potential can be written as

$$
\widehat{\xi}_{r}=\frac{\xi_{r}}{R}, \quad \widehat{P}_{1}=\frac{R^{4}}{G M^{2}} P_{1}, \quad \widehat{\Phi}_{1}=\frac{G M}{R} \Phi_{1} .
$$




\subsection{Properties of stellar oscillations}

Equations (35), (37) and (39) can appear rather opaque when it comes to understanding the properties of stellar oscillations. The way to go about trying to distil the properties is to apply a few simplifying assumptions.

The first such assumption is to assume that the perturbation to the gravitational potential, $\Phi_{1}$, can be ignored, i.e., the Cowling approximation can be applied. It can be shown that this approximation applies to modes of oscillation where $|n|$ or $\ell$ is large.

Applying the Cowling approximation reduces the equations to

$$
\frac{\mathrm{d} \xi_{r}}{\mathrm{~d} r}=-\left(\frac{2}{r}-\frac{1}{\Gamma_{1}} \frac{1}{H_{p}}\right) \xi_{r}+\frac{1}{\rho c^{2}}\left(\frac{S_{\ell}^{2}}{\omega^{2}}-1\right) P_{1},
$$

and

$$
\frac{\mathrm{d} P_{1}}{\mathrm{~d} r}=\rho\left(\omega^{2}-N^{2}\right) \xi_{r}-\frac{1}{\Gamma_{1}} \frac{1}{H_{p}} P_{1}
$$

where

$$
H_{p}=-\frac{\mathrm{d} r}{\mathrm{~d} \ln P}
$$

is the pressure scale height.

Another assumption made is that we are looking far away from the centre (and we shall see soon that this applies to modes of high $\ell$ ). Under this condition the term $2 / r$ in the right-hand side of Eq. (50) can be neglected. The third assumption is that for high $|n|$ oscillations, the eigenfunctions vary much more rapidly than the equilibrium quantities. This assumption applies away from the stellar surface. The implication of this assumption is that terms containing $H_{p}^{-1}$ in Eqs. (50) and (51) can be neglected when compared with the quantities on the left hand sides of the two equations. Thus, Eqs. (50) and (51) reduce to

$$
\frac{\mathrm{d} \xi_{r}}{\mathrm{~d} r}=\frac{1}{\rho c^{2}} P_{1}
$$

and

$$
\frac{\mathrm{d} P_{1}}{\mathrm{~d} r}=\rho\left(\omega^{2}-N^{2}\right) \xi_{r}
$$

These two equations can be combined to form one second-order differential equation

$$
\frac{\mathrm{d}^{2} \xi_{r}}{\mathrm{~d} r^{2}}=\frac{\omega^{2}}{c^{2}}\left(1-\frac{N^{2}}{\omega^{2}}\right)\left(\frac{S_{\ell}^{2}}{\omega^{2}}-1\right) \xi_{r} .
$$

Equation (55) is the simplest possible approximation to equations of non-radial oscillations, but it is enough to illustrate some of the key properties. One can see immediately that the equation does not always have an oscillatory solution. The solution is oscillatory when (1) $\omega^{2}<S_{\ell}^{2}$, and $\omega^{2}<N^{2}$, or when (2) $\omega^{2}>S_{\ell}^{2}$, and $\omega^{2}>N^{2}$. The solution is exponential otherwise. 
Fig. 1 The propagation diagram for a standard solar model. The blue line is the buoyancy frequency, the red lines are the Lamb frequency for different degrees. The green solid horizontal line shows the region where a $200 \mu \mathrm{Hz}$ g mode can propagate. The pink dashed horizontal line shows where a $1000 \mu \mathrm{Hz} \ell=5$ p mode can propagate

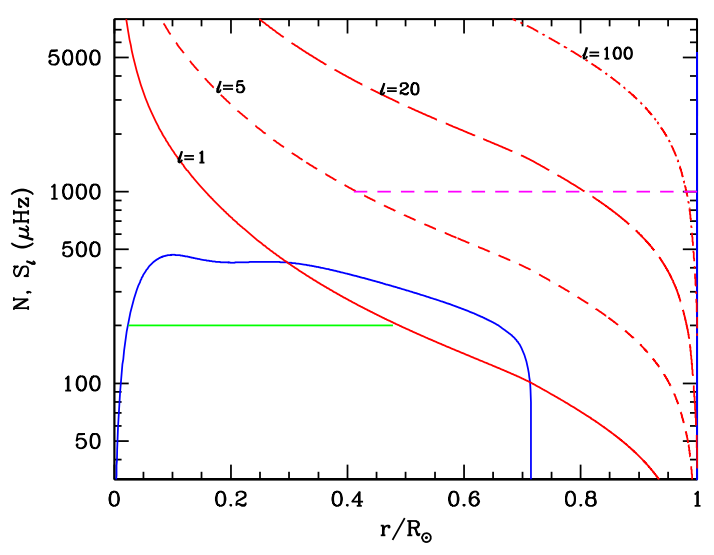

Equation (55) can be written in the form

$$
\frac{\mathrm{d}^{2} \xi_{r}}{\mathrm{~d} r^{2}}=K(r) \xi(r)
$$

where

$$
K(r)=\frac{\omega^{2}}{c^{2}}\left(1-\frac{N^{2}}{\omega^{2}}\right)\left(\frac{S_{\ell}^{2}}{\omega^{2}}-1\right) .
$$

In Fig. 1, we show $N^{2}$ and $S_{\ell}^{2}$ plotted as a function of depth for a model of the presentday Sun. Such a figure is often referred to as a "propagation diagram." The figure shows that for modes for which the first condition is true, i.e., $\omega^{2}<S_{\ell}^{2}$, and $\omega^{2}<N^{2}$, are trapped mainly in the core (since $N^{2}$ is negative in convection zones, and the Sun has an envelope convection zone). These are the $g$ modes and their restoring force is gravity through buoyancy. Modes that satisfy the second condition, i.e., $\omega^{2}>S_{\ell}^{2}$, and $\omega^{2}>N^{2}$, are oscillatory in the outer regions, though low-degree modes can penetrate right to the centre. These are the $p$ modes and their restoring force is predominantly pressure. One can see that $\mathrm{p}$ modes of different degrees penetrate to different depths within the Sun. High degree modes penetrate to shallower depths than low degree modes. For a given degree, modes of higher frequency penetrate deeper inside the star than modes of lower frequencies. Thus, modes of different degrees sample different layers of a star. Note that high- $\ell$ modes are concentrated in the outer layers, justifying to some extent the neglect of the $2 / r$ term in Eq. (50).

\subsubsection{P modes}

As mentioned above, $\mathrm{p}$ modes have frequencies with $\omega^{2}>S_{\ell}^{2}$ and $\omega^{2}>N^{2}$. The modes are trapped between the surface and a lower or inner turning point $r_{t}$ given by $\omega^{2}=S_{\ell}^{2}$, i.e.,

$$
\frac{c_{2}^{2}\left(r_{t}\right)}{r_{t}^{2}}=\frac{\omega^{2}}{\ell(\ell+1)} .
$$

This equation can be used to determine $r_{t}$ for a mode of given $\omega$ and $\ell$. 
For high frequency p modes, i.e., modes with $\omega \gg N^{2}, K(r)$ in Eq. (57) can be approximated as

$$
K(r) \simeq \frac{\omega^{2}-S_{\ell}^{2}(r)}{c^{2}(r)},
$$

showing that the behaviour of high-frequency p modes is determined predominantly by the behaviour of the sound-speed profile, which is not surprising since these are pressure, i.e., sound waves. The dispersion relation for sound waves is $\omega^{2}=c^{2}|k|^{2}$ where $\mathbf{k}$ is the wave-number that can be split into a radial and a horizontal parts, and $k^{2}=k_{r}^{2}+k_{h}^{2}$, where $k_{r}$ is the radial wavenumber, and $k_{h}$ the horizontal one. At the lower turning point, the wave has no radial component and hence, the radial part of the wavenumber, $k_{r}$, vanishes, which leads to

$$
k_{r}^{2}=\frac{\omega^{2}-S_{\ell}^{2}(r)}{c^{2}(r)},
$$

which immediately implies (and which can be derived rigorously) that

$$
k_{t}^{2}=\frac{\ell(\ell+1)}{r^{2}} .
$$

A better analysis of the equations (see Christensen-Dalsgaard 2003) shows that since we are talking of normal modes, there are further conditions on $K(r)$. In particular, the requirement that the modes have a lower turning point $r_{t}$ and an upper turning point at $r_{u}$ requires

$$
\int_{r_{t}}^{r_{u}} K(r)^{1 / 2} \mathrm{~d} r=\left(n-\frac{1}{2}\right) \pi .
$$

We have the approximate expression for $K$ (Eq. 59) but the analysis that lead to it had no notion of an upper turning point. We just assume that the upper turning point is at $r=R$. Thus, the "reflection" at the upper turning point does not necessarily produce a phase-shift of $\pi / 2$, but some unknown shift which we call $\alpha_{p} \pi$. In other words

$$
\int_{r_{t}}^{R} K(r)^{1 / 2} \mathrm{~d} r=\int_{r_{t}}^{R}\left(\omega^{2}-S_{\ell}^{2}\right)^{1 / 2} \mathrm{~d} r=\left(n+\alpha_{p}\right) \pi .
$$

Since $\omega$ does not depend on $r$, Eq. (63) can be rewritten as

$$
\int_{r_{t}}^{R}\left(1-\frac{L^{2}}{\omega^{2}} \frac{c^{2}}{r^{2}}\right)^{1 / 2} \frac{\mathrm{d} r}{c}=\frac{\left(n+\alpha_{p}\right) \pi}{\omega}
$$

where $L=\sqrt{\ell(\ell+1)}$, though a better approximation is that $L=\ell+1 / 2$. The LHS of the equation is a function of $w \equiv \omega / L$, and the equation is usually written as

$$
F(w)=\frac{\left(n+\alpha_{p}\right) \pi}{\omega}
$$


Fig. 2 The Duvall Law for a modern dataset. The frequencies used in this figure are a combination of BiSON and MDI frequencies, specifically we have used modeset BiSON-13 of Basu et al. (2009). Note that all modes fall on a narrow curve for $\alpha_{p}=1.5$. The curve would have been narrower had $\alpha_{p}$ been allowed to be a function of frequency

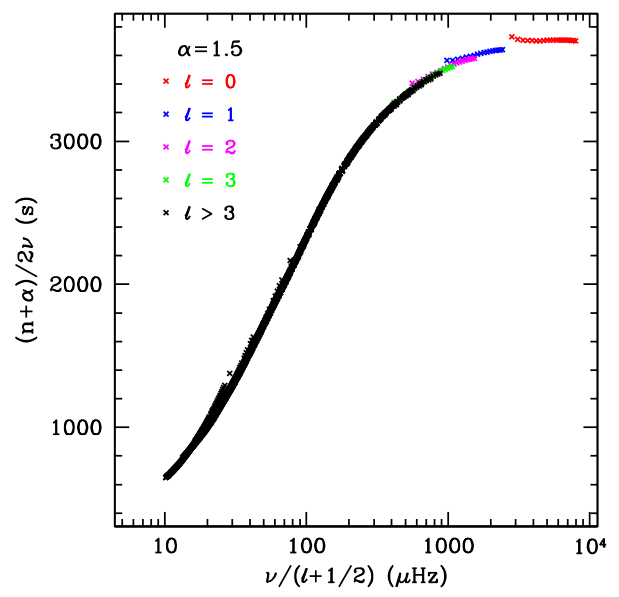

where

$$
F(w)=\int_{r_{t}}^{R}\left(1-\frac{L^{2}}{\omega^{2}} \frac{c^{2}}{r^{2}}\right)^{1 / 2} \frac{\mathrm{d} r}{c} .
$$

Eq. (65) is usually referred to as the 'Duvall Law'. Duvall (1982) plotted $\left(n+\alpha_{p}\right) \pi / \omega$ as a function of $w=\omega / L$ and showed that all observed frequencies collapse into a single function of $w$. As we can see from Eq. (58), $w$ is related to the lower turning point of a mode since

$$
w \equiv \frac{\omega}{\sqrt{\ell(\ell+1)}}=\frac{c^{2}\left(r_{t}\right)}{r_{t}} .
$$

A version of the Duvall-law figure with more modern data is shown in Fig. 2. As we shall see later, the Duvall law can be used to determine the solar sound-speed profile from solar oscillation frequencies.

A mathematically rigorous asymptotic analysis of the equations (see e.g., Tassoul 1980) shows that the frequency of high-order, low-degree p modes can be written as

$$
v_{n, \ell} \simeq\left(n+\frac{\ell}{2}+\alpha_{p}\right) \Delta v
$$

where

$$
\Delta v=\left(2 \int_{0}^{R} \frac{\mathrm{d} r}{c}\right)^{-1}
$$

is twice the sound-travel time of the Sun or star. The time it takes sound to travel from the surface to any particular layer of the star is usually referred to as the "acoustic depth." $\Delta v$ is often referred to as the "large frequency spacing" or "large frequency separation" and is the frequency difference between two modes of the same $\ell$ but consecutive values of $n$. Equation (68) shows us that p modes are equidistant in frequency. It can be shown that the large spacing scales as the square root of density (Ulrich 1986; Christensen-Dalsgaard 1988). The phase shift $\alpha_{p}$ however, is generally frequency dependent and thus the spacing between modes is not strictly a constant. 
A higher-order asymptotic analysis of the equations (Tassoul 1980, 1990) shows that

$$
v_{n, \ell} \simeq\left(n+\frac{\ell}{2}+\frac{1}{4}+\alpha_{p}\right) \Delta v-\left(A L^{2}-\delta\right) \frac{\Delta v^{2}}{v_{n, \ell}},
$$

where, $\delta$ is a constant and

$$
A=\frac{1}{4 \pi^{2} \Delta v}\left[\frac{c(R)}{R}-\int_{0}^{R} \frac{\mathrm{d} c}{\mathrm{~d} r} \frac{\mathrm{d} r}{r} .\right]
$$

When the term with the surface sound speed is neglected we get

$$
v_{n \ell}-v_{n-1, \ell+2} \equiv \delta v_{n, \ell} \simeq-(4 \ell+6) \frac{\Delta v}{4 \pi^{2} v_{n, \ell}} \int_{0}^{R} \frac{\mathrm{d} c}{\mathrm{~d} r} \frac{\mathrm{d} r}{r}
$$

$\delta v_{n \ell}$ is called the small frequency separation. From Eq. (72) we see that $\delta v_{n \ell}$ is sensitive to the gradient of the sound speed in the inner parts of a star. The sound-speed gradient changes with evolution as hydrogen is replaced by heavier helium making $\delta v_{n l}$ is a good diagnostic of the evolutionary stage of a star. For main sequence stars, the average value of $\delta v$ decreases monotonically with the central hydrogen abundance and can be used in various forms to calibrate age if metallicity is known (Christensen-Dalsgaard 1988; Mazumdar and Roxburgh 2003; Mazumdar 2005, etc.).

\subsubsection{G modes}

G modes are low frequency modes with $\omega^{2}<N^{2}$ and $\omega^{2}<S_{\ell}^{2}$. The turning points of these modes are defined by $N=\omega$. Thus, in the case of the Sun we would expect $\mathrm{g}$ modes to be trapped between the base of the convection zone and the core.

For $g$ modes of high order, $\omega^{2} \ll S_{\ell}^{2}$ and thus

$$
K(r) \simeq \frac{1}{\omega^{2}}\left(N^{2}-\omega^{2}\right) \frac{\ell(\ell+1)}{r^{2}} .
$$

In other words, the properties of $\mathrm{g}$ modes are dominated by the buoyancy frequency $N$. The radial wavenumber can be shown to be

$$
k_{r}^{2}=\frac{\ell(\ell+1)}{r}\left(\frac{N^{2}}{\omega^{2}}-1\right) .
$$

An analysis similar to the one for $\mathrm{p}$ modes show that for $\mathrm{g}$ modes frequencies are determined by

$$
\int_{r_{1}}^{r_{2}} L\left(\frac{N^{2}}{\omega^{2}}-1\right)^{1 / 2} \frac{\mathrm{d} r}{r}=\left(n-\frac{1}{2}\right) \pi,
$$

where, $r_{1}$ and $r_{2}$ mark the limits of the radiative zone. Thus

$$
\int_{r_{1}}^{r_{2}}\left(\frac{N^{2}}{\omega^{2}}-1\right)^{1 / 2} \frac{\mathrm{d} r}{r}=\frac{(n-1 / 2) \pi}{L}=G(w),
$$


an expression which is similar to that for p modes [Eq. (65)] but showing that the buoyancy frequency plays the primary role this case.

A complete asymptotic analysis of g modes (see Tassoul 1980) shows that the frequencies of high-order g modes can be approximated as

$$
\omega=\frac{L}{\pi\left(n+\ell / 2+\alpha_{g}\right)} \int_{r_{1}}^{r_{2}} N \frac{\mathrm{d} r}{r},
$$

where $\alpha_{g}$ is a phase that varies slowly with frequency. This shows that while p modes are equally spaced in frequency, $g$ modes are equally spaced in period.

\subsubsection{Remaining issues}

The analysis presented thus far does not state anything explicitly about the upper turning point of the modes. It is assumed that all modes get reflected at the surface. This is not really the case and is the reason for the inclusion of the unknown phase factors $\alpha_{p}$ and $\alpha_{g}$ in Eqs. (63) and (68). The other limitation is that we do not see any $\mathrm{f}$ modes in the analysis.

The way out is to do a slightly different analysis of the equations without neglecting the pressure and density scale heights but assuming that curvature can be neglected. Such an analysis was presented by Deubner and Gough (1984) who followed the analysis of Lamb (1932). They showed that under the Cowling approximation, one could approximate the equations of adiabatic stellar oscillations to

$$
\frac{\mathrm{d}^{2} \Psi}{\mathrm{d} r^{2}}+K^{2}(r) \Psi=0
$$

where $\Psi=\rho^{1 / 2} c^{2} \nabla \cdot \xi$, and the wavenumber $K$ is given by

$$
K^{2}(r)=\frac{\omega-\omega_{c}^{2}}{c^{2}}+\frac{\ell(\ell+1)}{r^{2}}\left(\frac{N^{2}}{\omega^{2}-1}\right),
$$

with

$$
\omega_{c}^{2}=\frac{c^{2}}{4 H_{\rho}^{2}}\left(1-2 \frac{\mathrm{d} H_{\rho}}{\mathrm{d} r}\right),
$$

where $H_{\rho}$ is the density scale height given by $H_{\rho}=-\mathrm{d} r / \mathrm{d} \ln \rho$. The quantity $\omega_{c}$ is known as the "acoustic cutoff" frequency. The radius at which $\omega=\omega_{c}$ defines the upper limit of the cavity for wave propagation and that radius is usually called the upper turning point of a mode. For isothermal atmospheres the acoustic cutoff frequency is simply $\omega_{c}=c g \rho / 2 P$ (see e.g., Balmforth and Gough 1990). Figure 3 shows the acoustic cutoff frequency for a solar model. Note that the upper turning point of low-frequency modes is much deeper than that of high-frequency modes. The effect of the location of the upper turning point of a mode is seen in the corresponding eigenfunctions as well-the amplitudes of the eigenfunctions decrease towards the surface. This results in higher mode-inertia normalised to the surface displacement 


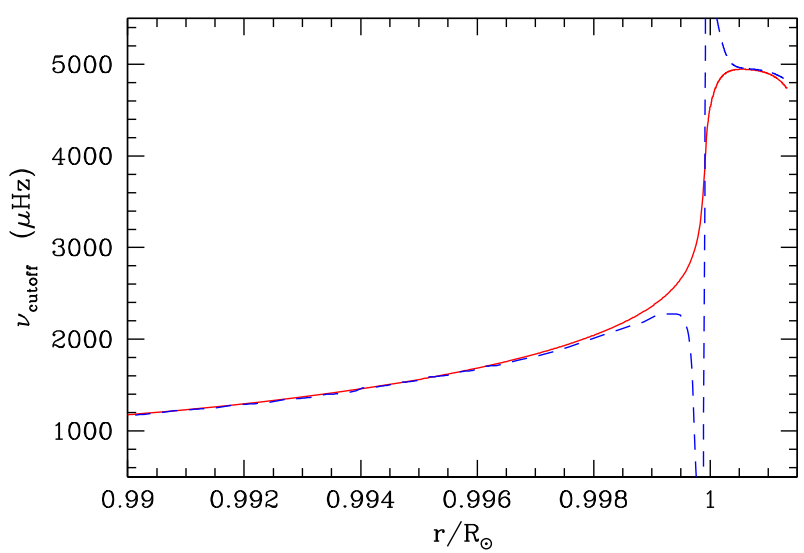

Fig. 3 The acoustic cut-off frequency of a solar model calculated as per Eq. (80) is shown as the blue dashed line. The red curve is the cut-off assuming that the model has an isothermal atmosphere. Note that the lower frequency modes would be reflected deeper inside the Sun than higher frequency modes

(see Eq. 43) for low-frequency modes compared to their high-frequency counterparts at the same value of $\ell$.

Eq. (78) can be solved under the condition $\Psi=0$. These are the f modes. It can be shown that the f-mode dispersion relation is

$$
\omega^{2} \simeq g k
$$

$k$ being the wavenumber. Thus, f-mode frequencies are almost independent of the stratification of the Sun. As a result, f-mode frequencies have not usually been used to determine the structure of the Sun. However, these have been used to draw inferences about the solar radius (e.g., Schou et al. 1997; Antia 1998; Lefebvre et al. 2007)

\section{A brief account of the history of solar models}

The history of solar models dates back to the 1940s. Among the first published solar models is that of Schwarzschild (1946). This model was constructed at a time when it was believed that the CNO cycle was the source of solar energy. By construction, this model did not have a convective envelope, but had a convective core instead. This model does not, of course, fall under the rubric of the standard solar model - that concept was not defined till much later. As the importance of the $p$ - $p$ chain came to be recognised, Schwarzschild et al. (1957) constructed models with the $p$ - $p$ chain as the source of energy; this model included a convective envelope. These models showed how the model properties depended on the heavy-element abundance and how the initial helium abundance could be adjusted to construct a model that had the correct luminosity. The central temperature and density of the models fall in the modern range, however, the adopted heavy element abundance is very different from what is observed now. In the intervening years, several others had constructed solar models assuming radiative envelopes and homogeneous compositions (see e.g., Epstein and Motz 1953; 
Naur 1954; Ogden Abell 1955); of course we now know that none of these models represent the Sun very well.

The 1960s saw a new burst of activity in terms of construction of solar models. The development of new numerical techniques such as the Henyey method for solving the stellar structure equations (Henyey et al. 1959) made calculations easier. An added impetus was provided by the development of methods to detect solar neutrinos (e.g., Davis 1955). This resulted in the construction of models to predict neutrino fluxes from the Sun, e.g., Pochoda and Reeves (1964), Sears (1964) and Bahcall et al. (1963). This was a time when investigations were carried out to examine how changes to input parameters change solar-model predictions (e.g., Demarque and Percy 1964; Ezer and Cameron 1965; Bahcall et al. 1968; Bahcall and Shaviv 1968; Iben 1968; Salpeter 1969; Torres-Peimbert et al. 1969). This was also the period when nuclear reaction rates and radiative opacities were modified steadily.

The 1970s and early 1980s saw the construction of solar models primarily with the aim of determining neutrino fluxes. This is when the term "standard solar model" was first used (see e.g., Bahcall and Sears 1972). It appears that the origin of the term was influenced by particle physicists working on solar neutrinos who, even at that time, had a standard model of particle physics (Pierre Demarque, private communication). The term "non-standard" models also came into play at this time. An example of an early non-standard model, and classified as such by Bahcall and Sears (1972), is that of Ezer and Cameron (1965) constructed with a time-varying gravitational constant $G$. Improvements in inputs to solar models led to many new solar models being constructed. Bahcall et al. (1982), Bahcall and Ulrich (1988) and Turck-Chièze et al. (1988) for instance looked at what happens to standard models when different microphysics inputs are changed. For a history of solar models from the perspective of neutrino physics, readers are referred to Bahcall (2003).

The 1980s was when helioseismic data began to be used to examine what can be said of solar models, and by extension, the Sun. Christensen-Dalsgaard and Gough (1980) compared frequencies of models to observations to show that none of the models examined was an exact match for the Sun. Bahcall and Ulrich (1988) compared the global seismic parameters of many models. During this time investigators also started examining how the p-mode frequencies of models change with model inputs. For instance, Christensen-Dalsgaard (1982) and Guenther et al. (1989) examined how the frequencies of solar models changed with change in opacity. This was also when the first solar models with diffusion of heavy elements were constructed (see e.g., Cox et al. 1989). Ever since it was demonstrated that the inclusion of diffusion increases the match of solar models with the Sun (Christensen-Dalsgaard et al. 1993), diffusion has become a standard ingredient of standard solar models.

Standard solar models have been constructed and updated continuously as different microphysics inputs have become available. Descriptions of many standard models have been published. Helioseismic tests of these models have helped examine the inputs to these models. Among published models are those of Bahcall and Pinsonneault (1992), Christensen-Dalsgaard et al. (1996), Guzik and Swenson (1997), Bahcall et al. (1995, 1998, 2005a, b), Guenther et al. (1996), Guenther and Demarque (1997), Brun et al. (1998), Basu et al. (2000a), Neuforge-Verheecke et al. (2001a, b), Couvidat et al. (2003), Bahcall and Serenelli (2005), etc. 
Many non-standard models have been constructed with a variety of motives. For instance, Ezer and Cameron (1965), as well as Roeder and Demarque (1966), constructed solar models with a time-varying value of the gravitational constant $G$ following the Brans-Dicke theory. More modern solar models with time-varying $G$ were those of Demarque et al. (1994) and Guenther et al. (1995) who were investigating whether solar oscillation frequencies could be used to constrain the time-variation of $G$. Christensen-Dalsgaard et al. (2005) on the other hand, tried to examine whether helioseismic data can constrain the value of $G$ given that $G M_{\odot}$ is known extremely precisely. Another set of non-standard models are ones that include early mass loss in the Sun. The main motivation for these models is to solve the so-called "faint Sun paradox". Models in this category include those of Guzik et al. (1987) and Sackmann and Boothroyd (2003).

A large number of non-standard models were constructed with the sole purpose of reducing the predicted neutrino flux from the models and thereby solving the solar neutrino problem (see Sect. 7.1 for a more detailed discussion of this issue). These include models with extra mixing (Bahcall et al. 1968; Schatzman 1985; Roxburgh 1985; Richard and Vauclair 1997, etc.). And some models were constructed to have low metallicity in the core with accretion of high- $Z$ materials to account for the higher metallicity at the surface (e.g., Christensen-Dalsgaard et al. 1979; Winnick et al. 2002). Models that included effects of rotation were also constructed (Pinsonneault et al. 1989).

More recently, non-standard solar models have been constructed to try and solve the problems solar models face if they are constructed with solar abundances as advocated by Asplund et al. (2005a, 2009). This issue has been reviewed in detail by Basu and Antia (2008) and more recent updates can be found in Basu and Antia (2013), and Basu et al. (2015). We discuss this issue in Sect. 7.5.

There is another class of solar models, the so-called 'seismic models' that have also been constructed. These models are constructed with helioseismically derived constraints in mind. We discuss those in Sect. 7.4.

\section{Frequency comparisons and the issue of the 'surface term'}

Like other fields in astronomy, as helioseismic data became available, researchers started testing how good their solar models were. This was done by comparing the computed frequencies of the models with the observed solar frequencies. Examples of this include Christensen-Dalsgaard and Gough (1980, 1981), Christensen-Dalsgaard et al. (1988a), etc. This practice continued till quite recently (e.g., Cox et al. 1989; Guzik and Cox 1991, 1993; Guenther et al. 1992; Sackmann and Boothroyd 2003, etc.). Such comparisons are of course quite common in the field of classical pulsators where $\mathrm{O}-\mathrm{C}$ (i.e., observed minus computed frequency) diagrams are considered standard. However, in the case of solar models such a comparison has pitfalls, as we discuss below.

In Fig. 4, we show the differences in frequencies of the Sun and the solar model Model S of Christensen-Dalsgaard et al. (1996). We see in Fig. 4a that the predominant frequency difference is a function of frequency, however, there is a clear dependence 

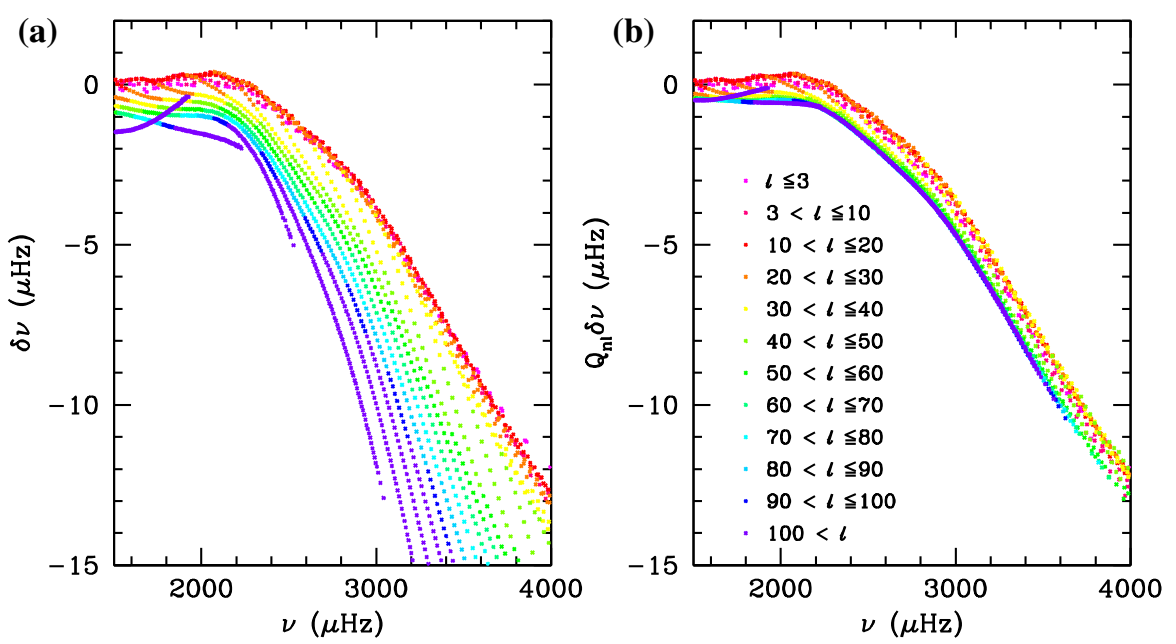

Fig. 4 The differences in frequencies of the Sun (mode set BiSON-13 of Basu et al. 2009) and that of standard solar model known as Model S (Christensen-Dalsgaard et al. 1996). a The raw frequency differences, $\mathbf{b}$ scaled differences, where the scaling factor $Q_{n \ell}$ corrects for the fact that modes with lower inertia change more for a given perturbation compared to modes with higher inertia

on degree. This $\ell$ dependence is mainly due to the fact that higher $\ell$ modes have lower mode inertia and hence, perturbed easily compared to modes of lower- $\ell$ (and hence higher inertia). This effect can be corrected for by scaling the frequency differences with their mode inertia. In practice, to ensure that both raw and scaled difference have the same order of magnitude, the differences are scaled with the quantity

$$
Q_{n \ell}=\frac{E_{n, \ell}(v)}{E_{\ell=0}(\nu)},
$$

where $E_{n, \ell}(\nu)$ is the mode inertia of a mode of degree $\ell$, radial order $n$ and frequency $v$ defined in Eq. (43), and $E_{\ell=0}(v)$ is the mode inertia of a radial mode at the same frequency. The quantity $E_{\ell=0}(v)$ is obtained by interpolation between the mode inertias of radial modes of different orders (and hence different frequencies). The scaled frequency-differences between the Sun and Model S can be seen in Fig. 4b. Note that most of the $\ell$-dependence disappears and one is left with frequency differences that are predominantly a function of frequency. If we were using these difference to determine whether Model S is a good model of the Sun, we could not draw much of a conclusion.

Similar issues are faced when we compare frequency-differences of different models with respect to the Sun. As an example in Fig. 5, we show the scaled frequency difference between the Sun and two models, one that does not include the diffusion and gravitational settling of helium and heavy elements and one that other; the remaining physics inputs are identical and the models were constructed with the same code. One can see that in both cases the predominant frequency difference is a function of frequency. There is a greater $\ell$ dependence in the model without diffusion, and this we know now is a result of the fact that the model without diffusion has a very shallow 

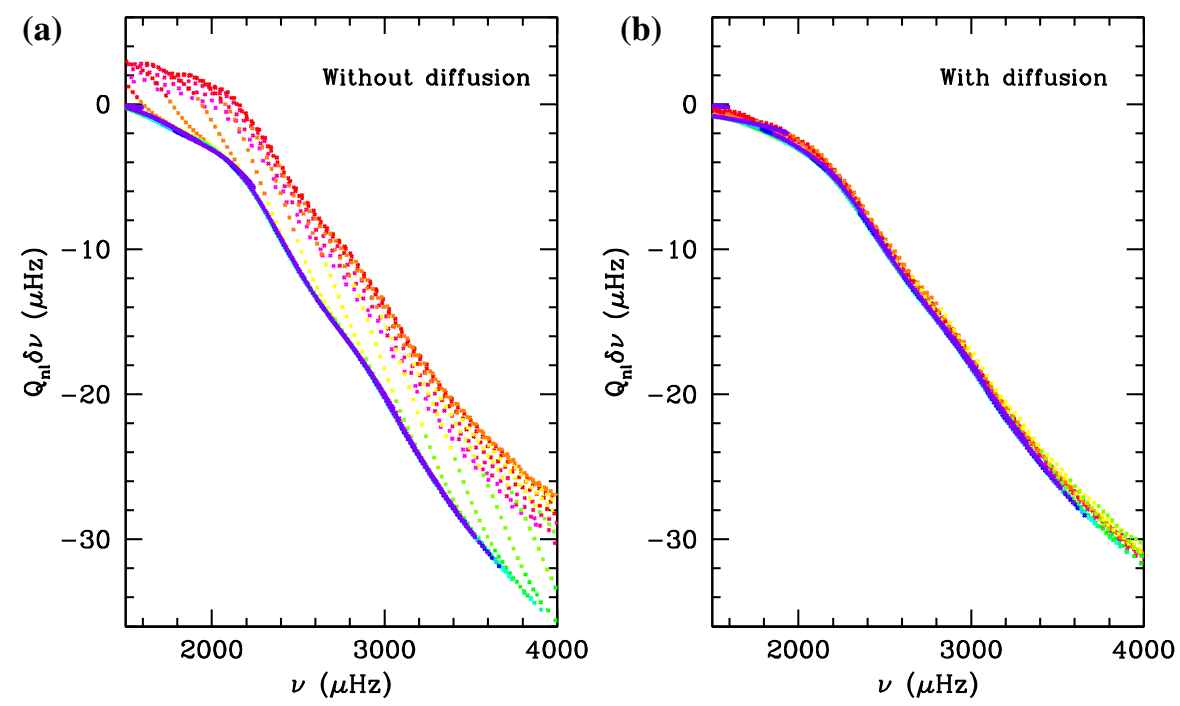

Fig. 5 The scaled frequencies differences between the Sun and two standard models, one without diffusion (a) and one with (b). The BiSON-13 mode set (Basu et al. 2009) is used for the Sun. The two models are models NODIF and STD of Basu et al. (2000a). Colours are the same as in Fig. 4

convection zone compared with the Sun. However, quantitatively, it is not possible to judge which model is better from these frequency differences alone. The $\chi^{2}$ per degree of freedom calculated for the frequency differences are extremely large (of the order of $10^{5}$ ) given the very small uncertainties in solar frequency measurements. Consequently, to try and draw some conclusion about which model is better, we look at the root-mean-square frequency difference instead. The root-mean-square frequency difference for the model with diffusion is $17.10 \mu \mathrm{Hz}$, while that for the model without diffusion is $17.03 \mu \mathrm{Hz}$. Thus, if the purpose of comparing frequencies was to determine which model is better compared with the Sun, we have failed. As we shall see later in Sect. 7.2, the model with diffusion is in reality the better of the two models in terms of match with the Sun.

Similar issues are faced if we are to compare other physics, such as the equation of state. In Fig. 6, we show the frequency difference between the Sun four models, each constructed with identical inputs, except for the equation of state. The EFF model has an RMS frequency difference of 5.5, the CEFF model of 4.8, the MHD model has an RMS difference of 5.3 and the OPAL model (this is Model S) has an RMS difference of 6.0. This we could be tempted to say that the CEFF model is the best and OPAL the worst, and the CEFF equation of state is the one we should use. However, as we shall see later, that is not the case: while CEFF is not too bad, OPAL is actually the best. In fact the spread in the differences of modes of different degrees is the clue to which model is better-generally speaking, the larger the spread, the worse the model. The models shown in Fig. 5 were constructed using a different code and different microphysics and atmospheric inputs than the models shown in Fig. 6, this gives rise to the large difference in the value of the RMS frequency differences for the 

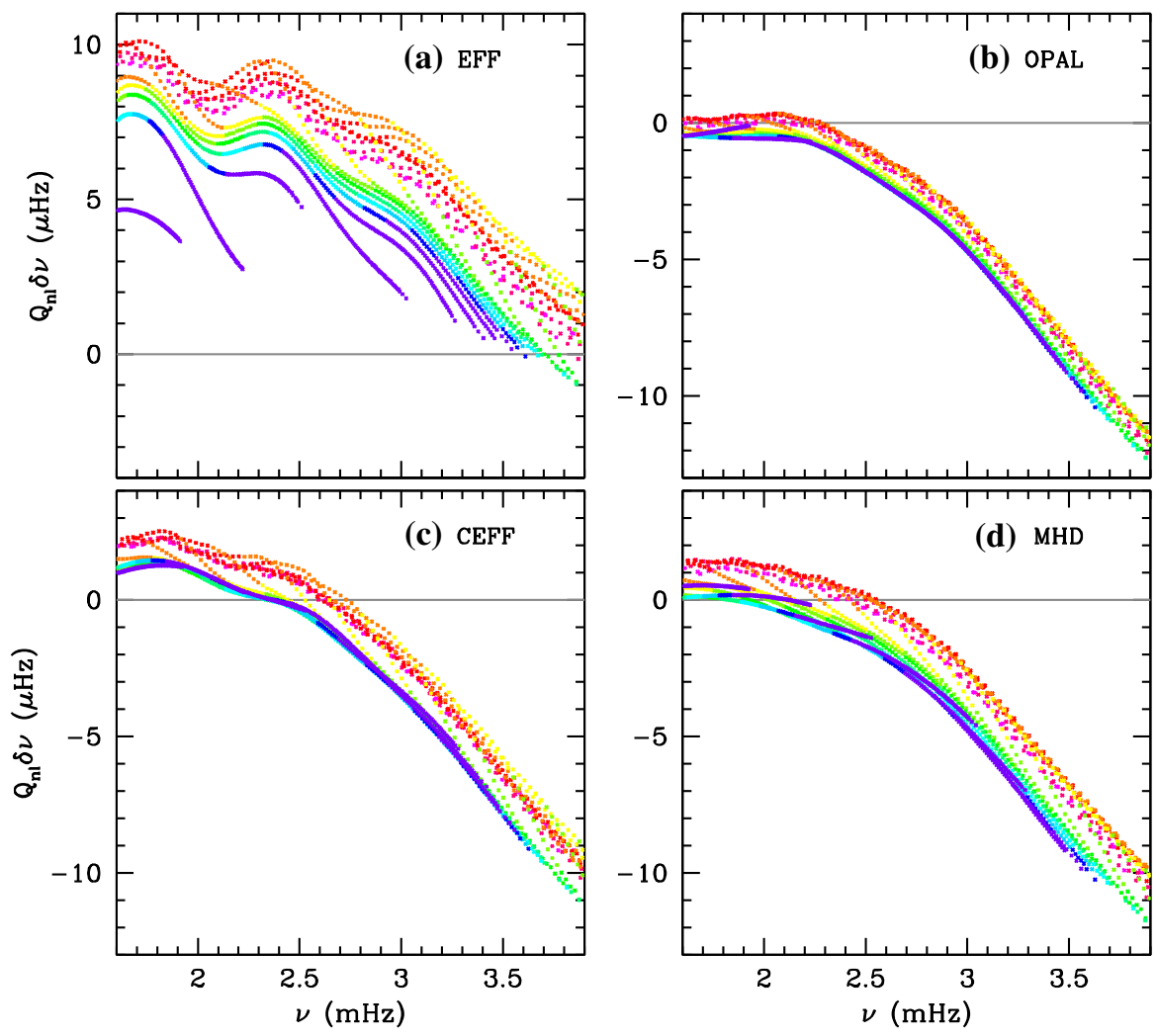

Fig. 6 The scaled frequency differences between the Sun and four models constructed with different equations of state but otherwise identical inputs. The OPAL model is Model S of Christensen-Dalsgaard et al. (1996). Colours are the same as in Fig. 4. (Models courtesy of J. Christensen-Dalsgaard)

different sets of models, again highlighting problems that make interpreting frequency differences difficult.

The reason behind the inability to use frequency comparisons to distinguish which of the two models is twofold. The first is that the frequencies of all the models were calculated assuming that the modes are fully adiabatic, when in reality adiabaticity breaks down closer to the surface. This of course, can be rectified by doing nonadiabatic calculations, as have been done by Christensen-Dalsgaard and Gough (1975), Guzik and Cox (1991, 1992), Guenther (1994), Rosenthal et al. (1995), etc. However, this is not completely straightforward since there is no consensus on how non-adiabatic calculations should be performed. The main uncertainty involves accounting for the influence of convection, and often damping by convection is ignored. The second reason is that there are large uncertainties in modelling the near-surface layers of stars. These uncertainties again include the treatment of convection. Models generally use the mixing length approximation or its variants and as a result, the region of inefficient convection close to the surface is modelled in a rather crude manner. The models do not include the dynamical effect of convection and pressure support due to 

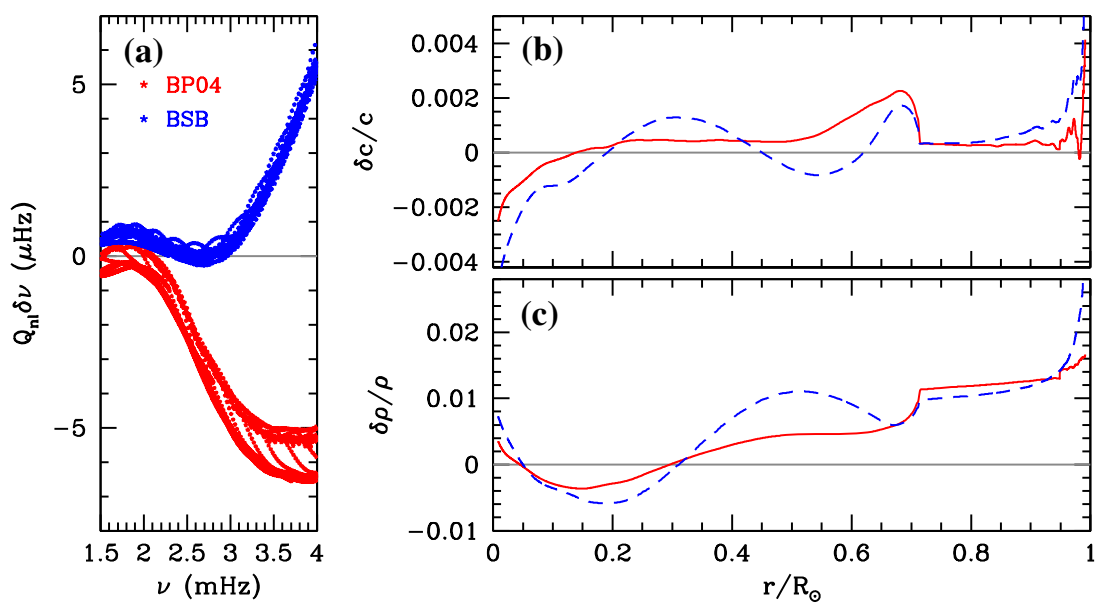

Fig. 7 a The scaled frequency differences between Model S and two other solar models. b The relative sound-speed differences, and $\mathbf{c}$ the relative density differences between the same models. All differences are in the sense (Model S-other model)

turbulence either; this is important again in the near surface layers. The treatment of stellar atmospheres is crude too, one generally uses simple atmospheric models such as the Eddington $T-\tau$ relation or others of a similar nature, and these are often, fully radiative, grey atmospheres and do not include convective overshoot from the interior. Some of the microphysics inputs can be uncertain too, in particular low-temperature opacities that have to include molecular lines as well as lines of elements that cause line-blanketing. Since all these factors are relevant in the near-surface regions, their combined effect is usually called the "surface effect" and the differences in frequencies they introduced is referred to as the "surface term." Even if we could calculate nonadiabatic frequencies properly and alleviate some of the problems, the issues with modelling will still give rise to a surface term.

The surface term also hampers the inter-comparison of models. Figure 7 shows the frequency differences between Model $\mathrm{S}$ and two other standard solar models, model BP04 of Bahcall et al. (2005a) and model BSB (GS98) (which we simply refer to as BSB) of Bahcall et al. (2006). Also shown are the relative sound-speed and density differences between Model S and the two models. As can be seen while the structural differences between Model S and two models are similar, the frequency differences are very different. Most of the frequency differences can be attributed to how the outer layers of the models were constructed.

For low and intermediate degree modes, the surface term depends only on the frequency of the mode once mode-inertia is taken into account. A simple way to visualise this would be to look at modes close to their upper turning points. In that regions, these modes travel almost radially, regardless of their degree. The only difference is brought about by the fact that modes of different frequencies are reflected at different layers in the near surface layers as was shown in Fig. 3. The effect of near-surface uncertainties is the least in low-frequency modes that have upper turning points deeper insider a star, and increases with mode-frequency since increasing mode frequencies push the 
upper turning point to progressively shallower layers were the uncertainties dominate. This is why the scaled frequency differences between Sun and the models increase with frequency. The fact that in Fig. 6 the EFF model shows large difference with respect to the Sun at low frequencies points to serious issues with the model in the deeper layers. However, this is not full picture. Christensen-Dalsgaard and Thompson (1997) investigated the reasons for the small frequency shifts in low-frequency modes that result from near-surface changes and challenged the view that the deeper upper-turning points of the low-frequency modes caused the small difference at low frequencies. They showed that the small shifts could be a result of near-cancellation of different contributions, which are individually much larger than the results shifts. The small shifts can be more easily explained if Lagrangian differences (i.e., differences at fixed mass), rather than Eulerian differences (differences at fixed radius) are considered; they found that the Lagrangian differences are indeed confined closer to the surface and explain the frequency shifts more naturally.

The surface term is not just any function of frequency, it is a smooth function of frequency that can be modelled as a low-degree polynomial. Gough (1990) showed that any localised feature in the sound speed inside a star introduces an oscillatory term in frequencies as a function of radial order $n$ which is proportional to

$$
\sin \left(2 \tau_{m} \omega_{n, \ell}+\phi\right)
$$

where, $\tau_{m}$ is the acoustic depth (i.e., the time it takes for sound to travel from the surface to a given layer) of the localised feature (usually referred to as an "acoustic glitch"), and

$$
\tau_{m}=\int_{r_{m}}^{R} \frac{\mathrm{d} r}{c},
$$

where $r_{m}$ is the radial position of the feature. As can be seen from Eq. (83), the smaller the acoustic depth of the glitch (i.e., the shallower the layer in which the glitch occurs), the larger the 'wavelength' of the frequency modulation. Our inability to model the near-surface layers of the Sun properly results in near-surface acoustic glitches in our models when compared with the Sun. Because these glitches arise at shallow depths, i.e., at small values of $\tau_{m}$, the effect on the frequency does not look like a sinusoidal modulation, but merely a smooth function that can be described as a low-order polynomial.

The smoothness of the surface term can be demonstrated by making models with different inputs that affect the near-surface layers. In Fig. 8, we show the scaled frequency differences between two standard solar models, one constructed with the Eddington $T-\tau$ relation and the other the Krishna Swamy $T-\tau$ relation. Also shown is the relative difference between the sound-speed profiles between the two models. Note that the sound-speed differences are significant only close to the surface, and that the frequency differences are smooth. Similar results are obtained when we have models with different formulations of convection but otherwise identical inputs (Fig. 9).

While the surface term is a nuisance, it is by no means devoid of information. Asymptotically, the influence of the upper layers of a star on p mode frequencies can be described as a phase function which essentially depends on the frequency of the 

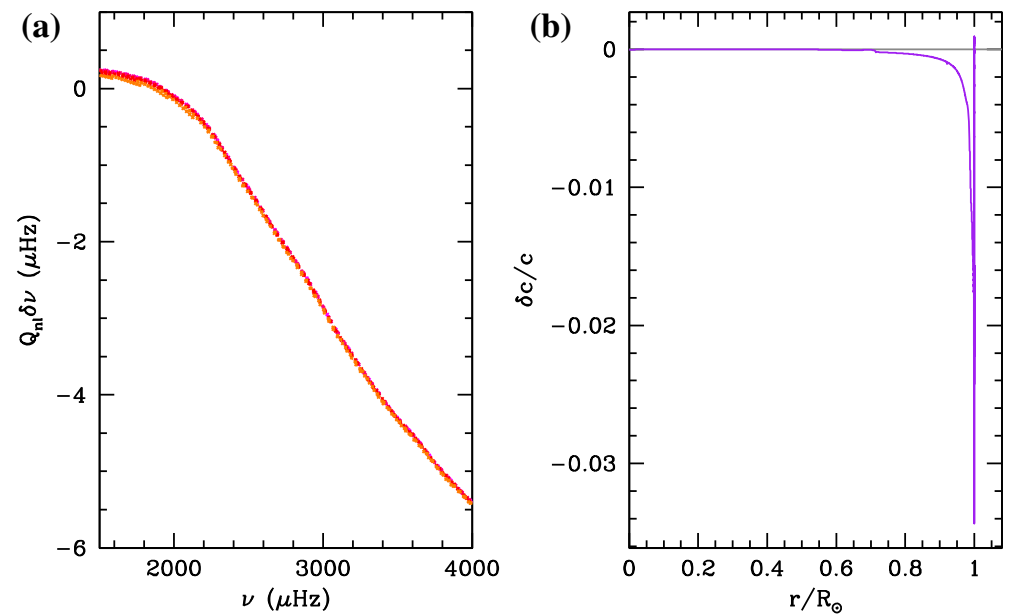

Fig. 8 a The scaled frequencies differences between a solar model constructed with the Eddington $T-\tau$ relation and one with the Krishna Swamy $T-\tau$ relation. Only differences of modes with $\ell<=50$ are shown. b The relative sound-speed difference between the two models. The differences are in the sense (Eddington-Krishna Swamy)
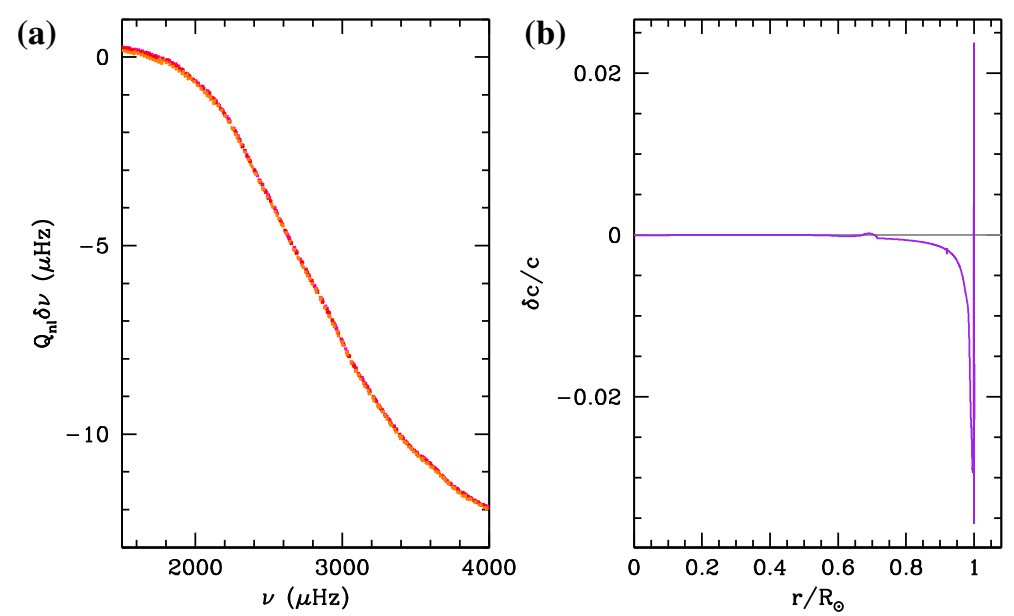

Fig. 9 a The scaled frequencies differences between a solar model constructed with the conventional mixing length treatment and one with the Canuto-Mazzitelli (CM) formulation. Only differences of modes with $\ell<=50$ are shown. b The relative sound-speed difference between the two models. The differences are in the sense (MLT-CM)

modes. Christensen-Dalsgaard and Pérez Hernández (1992), and Pérez Hernández and Christensen-Dalsgaard (1994a, b) did extensive studies of the surface term and derived kernels for the term relating it to near-surface differences in structure. Pérez Hernández and Christensen-Dalsgaard (1994b) even used it to determine the convection zone helium-abundance of the Sun. Antia and Basu (1994a), and Basu and Antia (1995) also used the surface term, though in a different manner, to determine the solar helium 
abundance; they however, found that the effect of the equation of state on the surface term resulted in systematic uncertainties in the estimate of the solar convection-zone helium abundance.

Since one of the major sources of the surface term is the treatment of convection, there have been attempts to change and improve the treatment of convection in solar models. Rosenthal et al. (1995) showed that the non-local mixing length formulation of (Gough 1977a) as applied by Balmforth (1992) reduces the surface term with respect to the Sun. These non-local formulations however, often use more than one free parameter, which is unsatisfactory. The most promising avenue however, seems to be to inclusion of dynamical effects from numerical simulations in models. Demarque et al. (1997) showed that by parameterizing the structure of the super-adiabatic layer seen in simulations and applying that to solar models improves frequencies. Instead of parameterizing the effects of convective dynamics, Rosenthal et al. (1998, 1999) attached their simulation results to models and showed that the surface term improves. This approach was also used by Piau et al. (2014) who, in addition to normal hydrodynamic simulations, also looked at the result of using magnetohydrodynamic simulations. This way of "pasting" the simulation results on a solar model however, does not allow one to construct evolutionary models of the Sun. Li et al. (2002) tried a different approach. They explicitly include the effects of turbulence in stellar structure equations and determined the quantities from simulations. However, this too has the problem that models can only be constructed for the present day Sun. Simulations of convection show that properties of stellar convection change with $\log g$ and $T_{\text {eff }}$ (Ludwig et al. 1999; Robinson et al. 2004; Tanner et al. 2013; Trampedach et al. 2013; Magic et al. 2013, etc.), thus a consistent treatment of realistic convection properties in solar models constructed from the zero-age main sequence is difficult.

The difficulty in drawing conclusions about models and their constituent microphysics by comparing frequencies have led to the development of other techniques, such as inversions, to determine solar structure and physics of the solar interior. Most helioseismic results are a result of inversions or similar techniques. In Sect. 6 we describe the common inversion techniques and some of the major results are described in Sect. 7.

\section{Inversions to determine interior structure}

The dominance of the surface effects in frequency differences between the Sun and solar models led to efforts in the direction of 'inverting' solar oscillation frequencies. The idea is simple: the frequencies of solar oscillations depend on the internal properties of the Sun in a known manner, thus if we had enough frequencies, we could determine solar structure from the frequencies.

Initial inversions for solar sound speed were done using the asymptotic expression given by Duvall Law (Eq. 65). However, limitations of the asymptotic expression and improvements in computational resources led to the development of inversion techniques using the full set of oscillation equations. We discuss both below. Earlier discussions of these techniques can be found in Gough and Thompson (1991), Thompson (1998a) and Christensen-Dalsgaard (2002). 


\subsection{Asymptotic inversions}

The first inversions were based on the Duvall Law. Gough (1984a) had shown that with a little mathematical manipulation, Eq. (66) can be re-written as

$$
w^{3} \frac{\mathrm{d} F}{\mathrm{~d} w}=\int_{w}^{a_{s}}\left(1-\frac{a^{2}}{w^{2}}\right)^{-1 / 2} \frac{\mathrm{d} \ln r}{\mathrm{~d} \ln a} \mathrm{~d} a,
$$

where $a \equiv c(r) / r$, and $a_{s}=a(R)$. This is an equation of the Abel type for which an analytic inverse exists:

$$
r=R \exp \left[\frac{-2}{\pi} \int_{a_{s}}^{a}\left(\frac{1}{w^{2}}-\frac{1}{a^{2}}\right)^{-1 / 2} \frac{\mathrm{d} F}{\mathrm{~d} w} \mathrm{~d} w\right] .
$$

The function $F(w)$ required in this equation can be obtained from observations using the Duvall Law by fitting a function (like a spline) though the points. The surface term can be accounted for by making $\alpha_{p}$ in Eq. (65) a function of frequency, though early inversions did not do so (see Christensen-Dalsgaard et al. 1985). One of the issues in this inversion is that it requires the value of $F(w)$ at the surface. Most observed modes have their lower-turning points well below the surface and hence $F(w)$ needs to be extrapolated. Inversions of frequencies of solar models also show that results near the core are unreliable. Brodsky and Vorontsov $(1987,1988)$ also used the Duvall Law as the starting point, but their way of determining $F(w)$ was different. They assumed that mode frequencies are a continuous function of $n$ and $L$ that can be determined from observations. They also used a frequency-dependent $\alpha_{p}$. Asymptotic inversions were also carried out by Kosovichev (1988b) who showed that the solar sound speed could be successfully determined between radii of 0.4 and $0.7 R_{\odot}$. Shibahashi (1988) and Sekii and Shibahashi (1989) had a different starting point: instead of using the Duvall Law, they used the quantisation condition obtained by Eq. (78). They did however, assume that $\omega^{2} \gg N^{2}$ and neglected the buoyancy frequency.

A breakthrough in the process of asymptotic inversions came when ChristensenDalsgaard et al. (1988c, 1989b) showed that more accurate (and precise) inversion results could be obtained if one linearised the equation for Duvall Law around a known solar model (a 'reference model') and inverted the frequency differences between the model and the Sun to determine the sound-speed difference between the model and the Sun. They perturbed Eq. (64) to show that, when one retained only terms linear in the perturbations, one gets

$$
S(w) \frac{\delta \omega}{\omega}=\int_{r_{t}}^{R}\left(1-\frac{c^{2}}{w^{2} r^{2}}\right)^{-1 / 2} \frac{\delta c}{c} \frac{\mathrm{d} r}{c}+\pi \frac{\delta \alpha}{\omega},
$$

where $S(w)$ is a function of the reference model, and is given by

$$
S(w)=\int_{r_{t}}^{R}\left(1-\frac{c^{2}}{w^{2} r^{2}}\right)^{-1 / 2} \frac{\mathrm{d} r}{c} .
$$


Note that $2 S(w)$ is the sound travel time along a ray between successive deflections at the surface. For properties of Eq. (87), see Christensen-Dalsgaard et al. (1988b). Note that the first term in the RHS of Eq. (87) is a function of $w$, while the second term is a function of $\omega$. The scaled frequency differences $S(w) \delta \omega / \omega$ can therefore be expressed as

$$
S(w) \frac{\delta \omega}{\omega}=H_{1}(w)+H_{2}(\omega) .
$$

Thus, the scaled frequency difference depend on the interior sound-speed difference through $H_{1}(w)$ and on differences at the surface through a function of $H_{2}(\omega)$ The function $H_{1}(w)$ is related to the sound speed by

$$
H_{1}(w)=\int_{\ln r_{t}(w)}^{\ln R}\left(1-\frac{a^{2}}{r^{2}}\right)^{-1 / 2} \frac{\delta c}{c} \frac{1}{a} \mathrm{~d} \ln r .
$$

This equation also has a closed-form inverse and one can show that (ChristensenDalsgaard et al. 1989b)

$$
\frac{\delta c}{c}=-\frac{2 a}{\pi} \frac{\mathrm{d}}{\mathrm{d} \ln r} \int_{a_{s}}^{a}\left(a^{2}-w^{2}\right)^{-1 / 2} H_{1}(w) \mathrm{d} w,
$$

or, alternatively (Basu and Antia 1994)

$$
\frac{\delta c}{c}=-\frac{2 r}{\pi} \frac{\mathrm{d} a}{\mathrm{~d} r} \int_{a_{s}}^{a} \frac{w}{\left(a^{2}-w^{2}\right)^{1 / 2}} \frac{\mathrm{d} H_{1}}{\mathrm{~d} w} \mathrm{~d} w .
$$

$H_{1}(w)$ and $H_{2}(\omega)$ can be obtained fitting one function of $w$ and one of $\omega$ to the frequency differences between the Sun and the reference model. Normally this is done by fitting splines (see, e.g., Christensen-Dalsgaard et al. 1989b; Basu and Antia 1994).

\subsection{Full inversions}

While asymptotic inversions, in particular differential asymptotic inversions, were successful in revealing some of the details of the solar interior, their inherent limitation was that they assumed that solar oscillations could be explained solely through differences in sound speed alone. The role of density perturbations was generally ignored. Although some attempts were made to include higher order terms that did not neglect the density perturbations (Vorontsov and Shibahashi 1991; Roxburgh and Vorontsov 1993), the methods did not become popular. Other limitations arise from the fact that the asymptotic relations generally assume that the eigenfunctions vary more rapidly than the pressure scale height, but this breaks down close to the surface. As a result, these days it is more common to do inversions based on the full set of equations. The increase in computing power also encouraged this change.

The equations of stellar oscillation derived earlier cannot be used to invert stellar frequencies to determine the interior structure. For that, we need to start with the perturbed form of the equation of motion, i.e., Eq. (22), i.e, 


$$
\rho \frac{\partial^{2} \boldsymbol{\xi}}{\partial t^{2}}=-\nabla P_{1}+\rho_{0} \mathbf{g}_{1}+\rho_{1} \mathbf{g} .
$$

As mentioned earlier, the displacement vector can be written as $\xi \exp (-i \omega t)$. Substituting this in the equation, we get

$$
-\omega^{2} \rho \xi=-\nabla P_{1}+\rho \mathbf{g}_{1}+\rho_{1} \mathbf{g},
$$

Substituting for $\rho_{1}$ from the perturbed continuity equation (Eq. 21) and $P_{1}$ from Eq. (25), we get

$$
-\omega^{2} \rho \boldsymbol{\xi}=\nabla\left(c^{2} \rho \nabla \cdot \boldsymbol{\xi}+\nabla P \cdot \boldsymbol{\xi}\right)-\mathbf{g} \nabla \cdot(\rho \boldsymbol{\xi})-G \rho \nabla\left(\int_{V} \frac{\nabla \cdot\left(\rho \boldsymbol{\xi} \mathrm{d}^{3} r\right.}{\left|\mathbf{r}-\mathbf{r}^{\prime}\right|}\right),
$$

where we have expressed the gravitational potential as an integral:

$$
\mathbf{g}_{1}=\nabla \Phi_{1}=\nabla \frac{\int_{V} \rho_{1} d^{3} r}{\left|\mathbf{r}-\mathbf{r}^{\prime}\right|}=-\nabla \int_{V} \frac{\nabla \cdot(\rho \boldsymbol{\xi}) \mathrm{d}^{3} r}{\left|\mathbf{r}-\mathbf{r}^{\prime}\right|}
$$

Equation (95) describes how the mode frequencies $\omega$ depend on the structure of the star and is the starting point for inversions. Note that $\xi \equiv \xi_{n, \ell}$ and $\omega \equiv \omega_{n, \ell}$, and thus there is one such equation for each mode described by eigenfunction $\xi_{n, \ell}$.

While Eq. (95) is the starting point of inversions, it is clear that the equation cannot be used as it stands. We can measure $\omega$ and we want to determine $c^{2}$ and $\rho$ (pressure $P$ is related to $\rho$ through the equation of hydrostatic equilibrium, and $\mathbf{g}$ can be derived from $\rho$ ), we do not have any means of determining the displacement eigenfunction $\boldsymbol{\xi}$ except at the surface. The way out of this stalemate is to recognise that Eq. (95) is an eigenvalue equation of the form

$$
\mathcal{L}\left(\xi_{n, \ell}\right)=-\omega_{n, \ell}^{2} \xi_{n, \ell},
$$

$\mathcal{L}$ being the differential operator in Eq. (95). Chandrasekhar (1964) showed that under specific boundary conditions, namely $\rho=P=0$ at the outer boundary, the eigenvalue problem defined by Eq. (95) is a Hermitian one. This allows us to move ahead.

If $\mathcal{O}$ be a differential Hermitian operator, and $x$ and $y$ be two eigenfunctions of the operator, then

$$
\int x^{*} \mathcal{O}(y) \mathrm{d} V=\int y \mathcal{O}\left(x^{*}\right) \mathrm{d} V,
$$

where * indicates a complex conjugate. This is often written as

$$
\langle x, \mathcal{O}(y)\rangle=\langle\mathcal{O}(x), y\rangle,
$$

where the operation \langle\rangle is usually called an inner product. The relevant inner product in the case of Eq. (95) is

$$
\langle\boldsymbol{\xi}, \boldsymbol{\eta}\rangle=\int_{V} \rho \boldsymbol{\xi}^{*} \cdot \boldsymbol{\eta} \mathrm{d}^{3} \mathbf{r}=4 \pi \int_{0}^{R}\left[\xi_{r}^{*}(r) \eta_{r}(r)+L^{2} \xi_{t}^{*}(r) \eta_{t}(r)\right] r^{2} \rho \mathrm{d} r .
$$


Hermitian operators have the property that their eigenvalues are real and that the variational principle applies. This means that if $a$ be the eigenvalue corresponding to the eigenfunction $x$, then

$$
a=\frac{\langle x, \mathcal{O}(x)\rangle}{\langle x, x\rangle} .
$$

Thus, if the frequency $\omega_{n, \ell}$ be the eigenvalue corresponding to the displacement eigenfunction $\xi_{n, \ell}$, then

$$
-\omega_{n, \ell}^{2}=\frac{\int_{V} \rho \boldsymbol{\xi}_{n, \ell}^{*} \cdot \mathcal{L}\left(\boldsymbol{\xi}_{n, \ell}\right) \mathrm{d}^{3} \mathbf{r}}{\int_{V} \rho \boldsymbol{\xi}_{n, \ell}^{*} \cdot \boldsymbol{\xi}_{n, \ell} \mathrm{d}^{3} \mathbf{r}} .
$$

Note that the denominator is the mode inertia [Eq. (42)].

The Hermitian nature of Eq. (95) allows us to use the variational principle to put the equation in the form that can be inverted. Equation (97) is first linearised around a known model, a reference model. Thus, if $\mathcal{L}$ be the operator for a model, we assume that the operator for the Sun or other star can be expressed as $\mathcal{L}+\delta \mathcal{L}$. The corresponding displacement eigenfunctions are $\xi$ and $\xi+\delta \xi$ respectively and the corresponding frequencies are respectively $\omega$ and $\omega+\delta \omega$. Thus

$$
(\mathcal{L}+\delta \mathcal{L})(\xi+\delta \xi)=-(\omega+\delta \omega)^{2}(\xi+\delta \xi),
$$

which on expansion and retention of only linear terms gives

$$
\begin{aligned}
& \int \xi^{*} \mathcal{L} \xi \mathrm{d} V+\int \xi^{*} \delta \mathcal{L} \xi \mathrm{d} V+\int \xi^{*} \mathcal{L} \delta \xi \mathrm{d} V+\int \xi^{*} \delta \mathcal{L} \delta \xi \mathrm{d} V=-\omega^{2} \int \xi^{*} \xi \mathrm{d} V \\
& -\omega^{2} \int \xi^{*} \delta \xi \mathrm{d} V-\left(\delta \omega^{2}\right) \int \xi^{*} \xi \mathrm{d} V-2 \omega \delta \omega \int \xi^{*} \xi \mathrm{d} V
\end{aligned}
$$

Because $\mathcal{L}$ is a Hermitian operator, all but two terms in Eq. (104) cancel out to give

$$
\int \xi^{*} \delta \mathcal{L} \xi \mathrm{d} V=-2 \omega \delta \omega \int \xi^{*} \xi \mathrm{d} V
$$

or

$$
\frac{\delta \omega}{\omega}=-\frac{\int_{V} \rho \boldsymbol{\xi} \cdot \delta \mathcal{L} \boldsymbol{\xi} \mathrm{d}^{3} \mathbf{r}}{2 \omega^{2} \int_{V} \rho \boldsymbol{\xi} \cdot \boldsymbol{\xi} \mathrm{d}^{3} \mathbf{r}}=-\frac{\int_{V} \rho \boldsymbol{\xi} \cdot \delta \mathcal{L} \boldsymbol{\xi} \mathrm{d}^{3} \mathbf{r}}{2 \omega^{2} E_{n, \ell}} .
$$

One such equation can be written for each mode, and these are the equations that we invert. Since the mode inertia appears in the denominator of Eq. (106), the equation indicates that for a given perturbation, frequencies of modes with lower inertia will change more than those with higher inertia.

\subsubsection{Inversions kernels}

In order to make further progress, we need to determine what $\delta \mathcal{L}$ is. For that we need to return to Eq. (95) and perturb it, keeping only terms linear in the perturbations. Thus, 


$$
\begin{aligned}
\delta \mathcal{L} \xi= & \nabla\left(\delta c^{2} \nabla \cdot \boldsymbol{\xi}+\delta \mathbf{g} \cdot \boldsymbol{\xi}\right)+\nabla\left(\frac{\delta \rho}{\rho}\right) c^{2} \nabla \cdot \boldsymbol{\xi}+\frac{1}{\rho} \nabla \rho \delta c^{2} \nabla \cdot \boldsymbol{\xi}+\delta \mathbf{g} \nabla \cdot \boldsymbol{\xi} \\
& -G \nabla \int_{V} \frac{\nabla \cdot(\delta \rho \boldsymbol{\xi})}{\left|\mathbf{r}-\mathbf{r}^{\prime}\right|} \mathrm{d}^{3} \mathbf{r}^{\prime}
\end{aligned}
$$

where $\delta c^{2}, \delta \mathbf{g}$ and $\delta \rho$ are the differences in sound speed, acceleration due to gravity and density between the reference model and the Sun. The quantity $\delta \mathbf{g}$ can be expressed in terms of $\delta \rho$. Following Antia and Basu (1994b), we substitute Eq. (107) in Eq. (106) and rearrange the terms to get:

$$
\frac{\delta \omega}{\omega}=-\frac{1}{2 \omega^{2} E}\left(I_{1}+I_{2}+I_{3}+I_{4}\right)
$$

where $E$ is the mode inertia and

$$
\begin{aligned}
I_{1} & =-\int_{0}^{R} \rho(\nabla \cdot \xi)^{2} \delta c^{2} r^{2} \mathrm{~d} r \\
I_{2} & =\int_{0}^{R} \xi_{r}[\rho \nabla \cdot \xi+\nabla \cdot(\rho \xi)] \delta g r^{2} \mathrm{~d} r, \quad \text { where } \\
\delta g(r) & =\frac{4 \pi G}{r^{2}} \int_{0}^{r} \delta \rho(s) s^{2} \mathrm{~d} s \\
I_{3} & =\int_{0}^{R} \rho c^{2} \xi_{r} \nabla \cdot \xi \frac{\mathrm{d}}{\mathrm{d} r}\left(\frac{\delta \rho}{\rho}\right) r^{2} \mathrm{~d} r
\end{aligned}
$$

and

$I_{4}=\frac{4 \pi G}{2 \ell+1} \int_{0}^{R} \nabla \cdot(\rho \xi) r^{2} \mathrm{~d} r\left[\frac{1}{r^{\ell+1}} \int_{0}^{r} s^{\ell+2}\left(\rho \nabla \cdot \xi-\rho \frac{d \xi_{r}}{\mathrm{~d} s}-\frac{\ell+2}{s} \rho \xi_{r}\right) \frac{\delta \rho}{\rho} \mathrm{d} s\right]$.

One can rewrite Eq. (109) in terms of $\delta c^{2} / c^{2}$, the relative difference between the squared sound speed of the Sun and the reference model. Similarly Eqs. (110)-(112) can be rewritten in terms of the relative density difference $\delta \rho / \rho$. In some cases this requires us to change the order of the integrals, as has been explained in Gough and Thompson (1991) and Gough (1993). Thus, Eq. (108) for each mode $i \equiv(n, \ell)$ can be written as

$$
\frac{\delta \omega_{i}}{\omega_{i}}=\int K_{c^{2}, \rho}^{i}(r) \frac{\delta c^{2}}{c^{2}}(r) \mathrm{d} r+\int K_{\rho, c^{2}}^{i}(r) \frac{\delta \rho}{\rho}(r) \mathrm{d} r .
$$

The terms $K_{c^{2}, \rho}^{i}(r)$ and $K_{\rho, c^{2}}^{i}(r)$ are known functions of the reference model and represent the change in frequency in response to changes in sound speed and density respectively. These two functions are known as the "kernels" of the inversion.

We show a few sound speed and density kernels in Figs. 10 and 11. Note that modes of higher $\ell$ are restricted closer to the surface, while modes of lower $\ell$ penetrate deeper. This is consistent with our earlier discussion of the lower turning points of different 


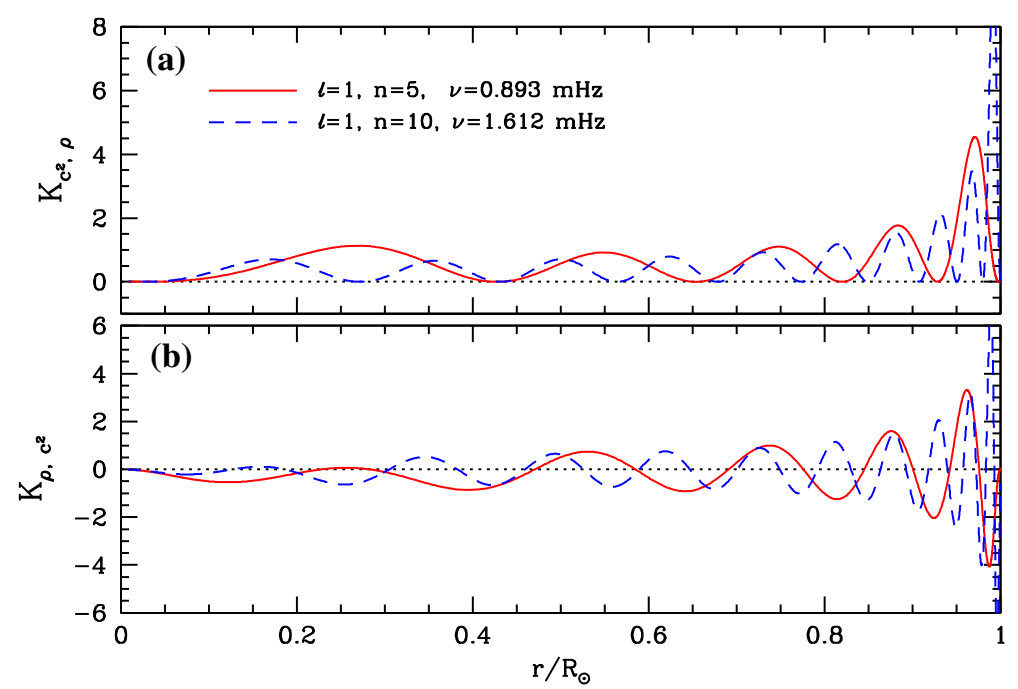

Fig. 10 Sound-speed kernels (a) and density kernels (b) for modes of the same degree but different radial orders (and hence frequencies)

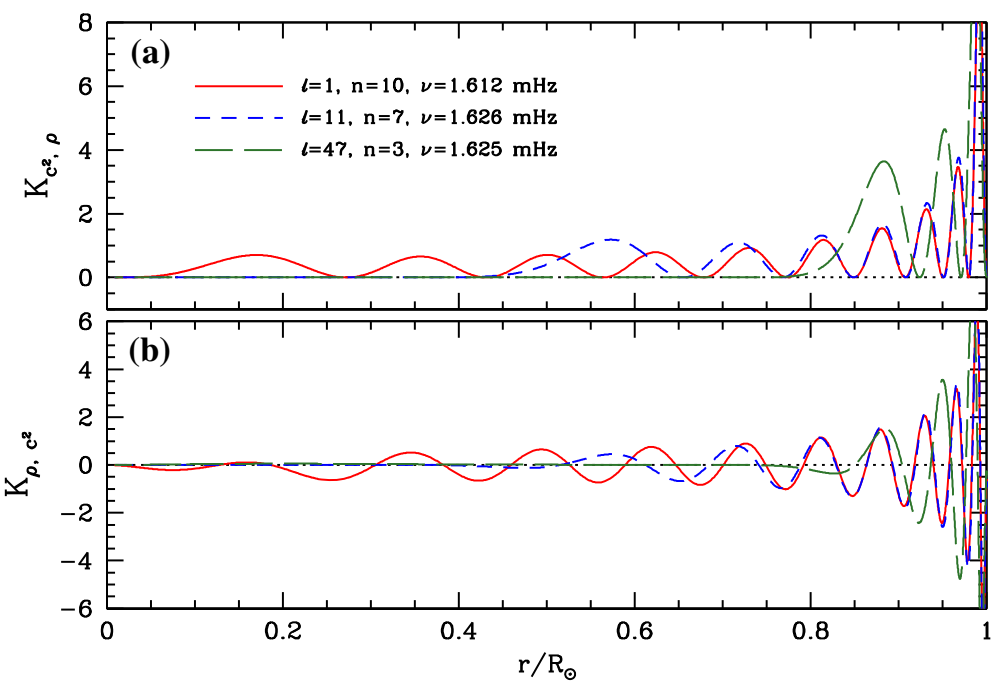

Fig. 11 Sound-speed kernels (a) and density (b) for modes of different degrees but similar frequencies. Note that modes of lower degrees penetrate deeper than modes of higher degrees

modes. Also note that sound-speed kernels are positive at all radii, while density kernels oscillate between positive and negative values. This oscillation reduces the contribution of the second term of the RHS of Eq. (113) and explains why density inversions are more difficult than sound speed inversions. This also explains why we can invert frequencies reasonably when using the asymptotic form of the oscillation equations where it is assumed that the frequency differences between a model and the Sun can be explained by differences in sound speed alone. The small discrepancies 
in the results obtained using asymptotic relation is a result of ignoring the small contribution from density.

The kernels for sound speed and density can be converted to those of other quantities (see Gough 1993). It is relatively straightforward to change the $\left(c^{2}, \rho\right)$ kernels to those for $\left(\Gamma_{1}, \rho\right)$. Since $c^{2}=\Gamma_{1} P / \rho$,

$$
\frac{\delta c^{2}}{c^{2}}=\frac{\delta \Gamma_{1}}{\Gamma_{1}}+\frac{\delta P}{P}-\frac{\delta \rho}{\rho} .
$$

Substituting Eq. (114) in Eq. (113) we get

$$
\frac{\delta \omega_{i}}{\omega_{i}}=\int K_{c^{2}, \rho}^{i}(r) \frac{\delta \Gamma_{1}}{\Gamma_{1}} \mathrm{~d} r+\int K_{c^{2}, \rho}^{i} \frac{\delta P}{P} \mathrm{~d} r+\int\left(K_{\rho, c^{2}}^{i}-K_{c^{2}, \rho}^{i}\right) \frac{\delta \rho}{\rho} \mathrm{d} r .
$$

Thus, $K_{\Gamma_{1}, \rho}=K_{c^{2}, \rho}$. To obtain the $K_{\rho, \Gamma_{1}}$, the equation of hydrostatic equilibrium, $d P / \mathrm{d} r=-g \rho$, has to be used to write $\int K_{c^{2}, \rho}(\delta P / P) \mathrm{d} r$ in terms of $\delta \rho / \rho$ though an integral. Using that and changing the order of integrations, we can rewrite

$$
\int K_{c^{2}, \rho}(\delta P / P) \mathrm{d} r-\int K_{c^{2}, \rho}(r)(\delta \rho / \rho) \mathrm{d} r+\int K_{\rho, c^{2}}(\delta \rho / \rho) \mathrm{d} r=\int B(r)(\delta \rho / \rho) \mathrm{d} r,
$$

where $B(r)$ is the kernel $K_{\rho, \Gamma_{1}}$.

Closed-form kernels are not possible for variable pairs other than $\left(c^{2}, \rho\right)$ and $\left(\Gamma_{1}, \rho\right)$. For instance, going from $\left(c^{2}, \rho\right)$ to $\left(u, \Gamma_{1}\right)(u \equiv P / \rho)$, we find that $K_{\Gamma_{1}, u} \equiv K_{c^{2}, \rho}$ (from Eq. 114), but the second kernel of the pair, $K_{u, \Gamma_{1}}$, does not have a closed form solution. It can be written as

$$
K_{u, \Gamma_{1}}=K_{c^{2}, \rho}-P \frac{\mathrm{d}}{\mathrm{d} r}\left(\frac{\psi}{P}\right),
$$

where, $\psi$ is a solution of the equation

$$
\frac{\mathrm{d}}{\mathrm{d} r}\left(\frac{P}{r^{2} \rho} \frac{d \psi}{\mathrm{d} r}\right)-\frac{\mathrm{d}}{\mathrm{d} r}\left(\frac{G m}{r^{2}} \psi\right)+\frac{4 \pi G \rho}{r^{2}} \psi=-\frac{\mathrm{d}}{\mathrm{d} r}\left(\frac{P}{r^{2}} F\right)
$$

for $F=K_{\rho, c^{2}}$.

It has also been common to use the knowledge of the equation of state of stellar matter to inject some more information into the system and convert $\left(\Gamma_{1}, \rho\right)$ kernels to kernels of $(u, Y), Y$ being the helium abundance. Kernels for $Y$ are non-zero only in the ionisation zone, which makes inversions easier. The adiabatic index $\Gamma_{1}$ can be expressed as

$$
\begin{aligned}
\frac{\delta \Gamma_{1}}{\Gamma_{1}} & =\left(\frac{\partial \ln \Gamma_{1}}{\partial \ln P}\right)_{\rho, Y} \frac{\delta P}{P}+\left(\frac{\partial \ln \Gamma_{1}}{\partial \ln \rho}\right)_{P, Y} \frac{\delta \rho}{\rho}+\left(\frac{\partial \ln \Gamma_{1}}{\partial Y}\right)_{\rho, P} \delta Y \\
& \equiv \Gamma_{1, P} \frac{\delta P}{P}+\Gamma_{1, \rho} \frac{\delta \rho}{\rho}+\Gamma_{1, Y} \delta Y
\end{aligned}
$$


where the partial derivatives can be calculated using the equation of state. Once we know this, it is easy to see that

$$
K_{Y, u} \equiv \Gamma_{1, Y} K_{\Gamma_{1}, \rho}, \quad \text { and } \quad K_{u, Y} \equiv \Gamma_{1, P} K_{\Gamma_{1}, \rho}-P \frac{\mathrm{d}}{\mathrm{d} r}\left(\frac{\psi}{P}\right),
$$

with $\psi$ being the solution of Eq. (118) with $F \equiv\left(\Gamma_{1, P}+\Gamma_{1, \rho}\right) K_{\Gamma_{1}, \rho}+K_{\rho, \Gamma_{1}}$. In a similar way, it is possible to derive kernels for the stability criterion $A^{*}$ given by

$$
A^{*}(r)=\frac{1}{\Gamma_{1}} \frac{\mathrm{d} \ln P}{\mathrm{~d} \ln r}-\frac{\mathrm{d} \ln \rho}{\mathrm{d} \ln r}
$$

(e.g., Kosovichev 1990; Elliott 1996).

\subsubsection{Taking care of the surface term}

Unfortunately, Eq. (113) does not take into account the issue of the surface term that was discussed earlier in Sect. 5. Equation (106) implies that we can invert the frequencies provided we know how to model the Sun properly, and provided that the frequencies can be described by the equations for adiabatic oscillations, which we know from Sect. 5, is not the case at all. For modes that are not of very high degree ( $\mathrm{p}$ modes of $\ell \simeq 200$ and lower), the structure of the wavefront near the surface is almost independent of the degree of the modes. Thus, any additional frequency difference is a function of frequency alone once the effect of mode inertia has been taken into account. This leads us to modify Eq. (113) to represent the difference between the Sun and the reference model as

$$
\frac{\delta \omega_{i}}{\omega_{i}}=\int \mathcal{K}_{c^{2}, \rho}^{i}(r) \frac{\delta c^{2}}{c^{2}}(r) \mathrm{d} r+\int \mathcal{K}_{\rho, c^{2}}^{i}(r) \frac{\delta \rho}{\rho}(r) \mathrm{d} r+\frac{F\left(\omega_{i}\right)_{\text {surf }}}{E_{i}}
$$

where $F\left(\omega_{i}\right)_{\text {surf }}$ is a slowly varying function of frequency that represents the surface term (see e.g., Dziembowski et al. 1990; Antia and Basu 1994b, and references therein).

Using a surface correction of the form shown in Eq. (122) is equivalent to passing the frequency differences and kernels through a high-pass filter and thus filtering out a slowly-varying component from the frequency differences and the kernels (Basu et al. 1996a). Basu et al. (1996a) also showed that such a simple filtering is not sufficient when dealing with modes of a degree higher than about $\ell=200$ since the wavefront at the surface is not radial for these modes. Indeed, when using higher degree models, often an $\ell$-dependent second function is added to Eq. (122). Brodsky and Vorontsov (1993) developed this in the context of asymptotic inversions. This was explored further by Gough and Vorontsov (1995). The asymptotic expansion was adapted to the full-non-asymptotic inversions by Di Mauro et al. (2002) who used a surface term of the form

$$
F(\omega)=F_{1}(\omega)+F_{2}(\omega) w^{2}+F_{3}(\omega) w^{4}
$$


where as before $w \equiv \omega / L$. Antia (1995), looking at inversion results between two models, showed that a slightly different form of the surface term

$$
F(v) \equiv F_{0}(v)+\ell(\ell+1) F_{1}(v)+[\ell(\ell+1)]^{2} F_{2}(v) \ldots,
$$

also improves inversions with high-degree modes.

\subsection{Inversion techniques}

For $N$ observed modes, Eq. (122) represents $N$ equations that need to be "inverted" or solved to determine the sound-speed and density differences. The data $\delta \omega_{i} / \omega_{i}$ or equivalently $\delta v_{i} / \nu_{i}$ are known. The kernels $K_{c^{2}, \rho}^{i}$ and $K_{\rho, c^{2}}^{i}$ are known functions of the reference model. The task of the inversion process is to estimate $\delta c^{2} / c^{2}$ and $\delta \rho / \rho$ after somehow accounting for the surface term $F_{\text {surf }}$.

There is one inherent problem: no matter how many modes we observe, we only have a finite amount of data and hence a finite amount of information. The two unknowns, $\delta c^{2} / c^{2}$ and $\delta \rho / \rho$, being functions have an infinite amount for information. Thus, there is really no way that we can recover the two functions from the data. The best we may hope for is to find localised averages of the two functions at a finite number of points. How localised the averages are depends on the data we have and the technique used for the inversion. During the inversion process we often have to make additional assumptions, such as the sound-speed and density profiles of the Sun inferred from inversions should be positive, in order to get physically valid solutions. We might need to put other constraints too. For instance, when we invert oscillation data to determine the density profile we need to ensure that mass is conserved, i.e.,

$$
\int \frac{\delta \rho}{\rho} \rho(r) r^{2} \mathrm{~d} r=0 .
$$

This is usually done by defining an additional mode with:

$$
\omega=0, \quad K_{c^{2}, \rho}=0, \quad \text { and } \quad K_{\rho, c^{2}}=\rho(r) r^{2}
$$

One of the first prescriptions for numerically inverting solar oscillation frequencies using Eq. (122) was given by Gough (1985). Since then, many groups have inverted available solar frequencies to determine the structure of the Sun (Gough and Kosovichev 1993, 1998; Dziembowski et al. 1990, 1991, 1994; Däppen et al. 1991; Antia and Basu 1994b; Basu et al. 1996b, 1997, 2000b, 2009, etc.)

There are two popular methods of inverting Eq. (122): (1) The Regularised Least Squares (RLS) method, and (2) the method of Optimally Localised Averages (OLA). These are complementary techniques (see Sekii 1997, for a discussion) that have different aims. RLS aims to find the $\delta c^{2} / c^{2}$ and $\delta \rho / \rho$ profiles that give the best fit to the data (i.e., give the smallest residuals) while keeping the errors small; the aim of OLA is not to fit the data at all, but to find linear combinations of the frequency differences in such a way that the corresponding combination of kernels provides a 
localised average of the unknown function, again while keeping the errors small. We discuss the two inversion techniques in some detail below. Other descriptions may be found in Gough and Thompson (1991) and Basu (2014). Properties of inversion techniques were investigated by Christensen-Dalsgaard et al. (1990); although their investigation was specifically for inversions of solar dynamics, the general principles apply to structure inversions too.

\subsubsection{The regularised least squares technique}

RLS inversions start with expressing the three unknown functions in Eq. (122) in terms of well-defined basis functions. Thus,

$$
\begin{aligned}
F(\omega) & =\sum_{i=1}^{m} a_{i} \psi_{i}(\omega), \\
\frac{\delta c^{2}}{c^{2}} & =\sum_{i=1}^{n} b_{i} \phi_{i}(r), \\
\frac{\delta \rho}{\rho} & =\sum_{i=1}^{n} c_{i} \phi_{i}(r),
\end{aligned}
$$

where $\psi_{i}(\omega)$ are suitable basis functions in frequency $\omega$, and $\phi_{i}(r)$ are suitable basis functions in radius $r$. Thus, for $N$ observed modes, Eq. (122) represent $N$ equations of the form:

$$
\begin{aligned}
& b_{1} \int \phi_{1}(r) K_{c^{2}}^{i} \mathrm{~d} r+b_{2} \int \phi_{2}(r) K_{c^{2}}^{i} \mathrm{~d} r+\cdots+b_{n} \int \phi_{n}(r) K_{c^{2}}^{i} \mathrm{~d} r \\
& \quad+c_{1} \int \phi_{1}(r) K_{\rho}^{i} \mathrm{~d} r+c_{2} \int \phi_{2}(r) K_{\rho}^{i} \mathrm{~d} r+\cdots+c_{n} \int \phi_{n}(r) K_{\rho}^{i} \mathrm{~d} r \\
& +a_{1} \psi_{1}\left(\omega_{i}\right) / E_{i}+a_{2} \psi_{2}\left(\omega_{i}\right) / E_{i}+\cdots+a_{m} \psi_{m}\left(\omega_{i}\right) / E_{i}=\delta \omega_{i} / \omega_{i},
\end{aligned}
$$

where, for ease of writing we have denoted $K_{c^{2}, \rho}^{i}$ as $K_{c^{2}}^{i}$ and $K_{\rho, c^{2}}^{i}$ as $K_{\rho}^{i}$. What we need to do is find coefficients $a_{i}, b_{i}$ and $c_{i}$ such that we get the best fit to the data $\delta \omega_{i} / \omega_{i}$. This is done by minimising

$$
\chi^{2}=\sum_{i=1}^{N}\left(\frac{\frac{\delta \omega_{i}}{\omega_{i}}-\Delta \omega_{i}}{\sigma_{i}}\right)^{2}
$$

where $\Delta \omega_{i}$ represents the LHS of Eq. (128). While such a minimisation does give us a solution for the three unknown functions, the solutions often are usually extremely oscillatory in nature (see Fig. 12a). The main reason for this is that the data have errors and the oscillatory solution is a result of these errors propagating into solutions for the unknown function. The phenomenon is also related to the so-called 'Gibbs' Phenomenon' in mathematics and is a result of the fact that we only have a finite amount of data but are trying to recover infinite information in the form of three functions. To 


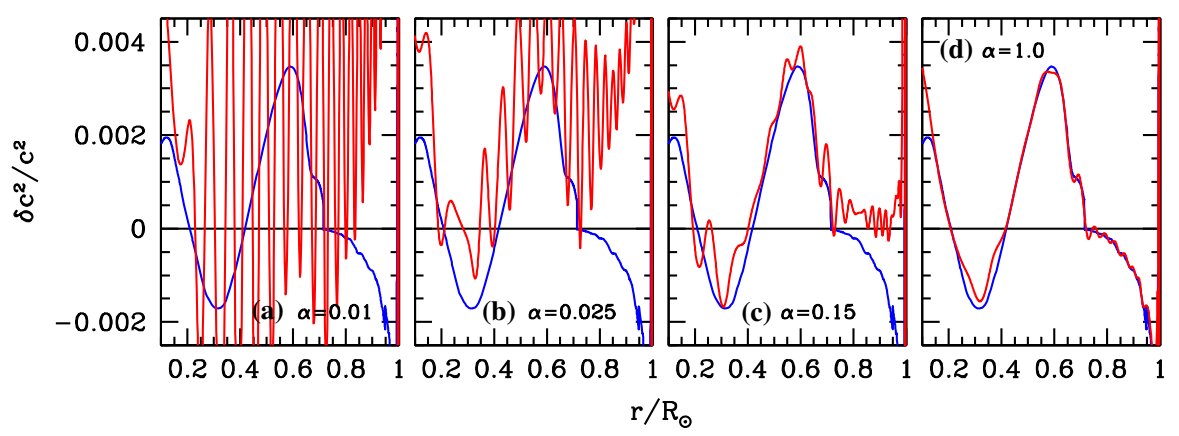

Fig. 12 The relative sound-speed differences between two solar models obtained by inverting their frequency differences using the RLS technique. In each panel the blue line is the true difference between the models and the red line is the inversion result. The different panels show the result obtained using different values of the smoothing parameter $\alpha$. The reference model used is model BP04 of Bahcall et al. (2005a) and the test model, or proxy Sun, is model BSB(GS98) of Bahcall et al. (2006). We only used those modes that have been observed in the Sun, specifically mode-set BiSON-13 of Basu et al. (2009). Random errors, corresponding to the uncertainties in the observed mode-set, were added to the data of the test model

ensure a more physical profile, 'regularisation' or 'smoothing' is applied (Craig and Brown 1986). This is implemented by demanding that we try to minimise the second derivative of the unknown functions while trying to minimise the $\chi^{2}$. Thus, instead of minimising Eq. (129), we minimise

$\chi_{\text {reg }}^{2}=\chi^{2}+\|L\|^{2}=\sum_{i=1}^{N}\left(\frac{\frac{\delta \omega_{i}}{\omega_{i}}-\Delta \omega_{i}}{\sigma_{i}}\right)^{2}+\alpha^{2} \int_{0}^{R}\left[\left(\frac{d^{2}}{\mathrm{~d} r^{2}} \frac{\delta \rho}{\rho}\right)^{2}+\left(\frac{d^{2}}{\mathrm{~d} r^{2}} \frac{\delta c^{2}}{c^{2}}\right)^{2}\right]$

where $\alpha$ is the regularisation or smoothing parameter. We could, if we wanted to, have different smoothing parameters for the two functions. The influence of the regularisation parameter on the solution can be seen in Fig. 12. Smoothing is not usually applied to the surface term $F(\omega)$, instead, the number and type of basis functions are chosen is such that $F(\omega)$ is a slowly varying function of frequency.

\section{Implementing RLS}

There are many ways in which the coefficients $a_{i}, b_{i}$ and $c_{i}$ may be determine. The implementation of Antia and Basu (1994b) and Basu and Thompson (1996) is discussed below.

Equation (128) clearly represents $N$ equations in $k=2 n+m$ unknowns. These equations can thus be represented as:

$$
A \mathbf{x}=\mathbf{d},
$$

where $A$ is an $N \times(2 n+m)$ matrix, $\mathbf{x}$ is a vector of length $(2 n+m)$ consisting of the unknown coefficients $a_{i}, b_{i}$ and $c_{i}$, and $\mathbf{d}$ is the vector with the data, i.e., $\delta \omega / \omega$. To take data errors into account, each row $i$ of each matrix is divided by $\sigma_{i}$, where $\sigma_{i}$ 
is the uncertainty of the $i$ th data point. The elements of vector $\mathbf{x}$ can be determined using singular value decomposition (SVD).

An SVD of matrix $A$ results in the decomposition of $A$ into three matrices, $A=$ $U \Sigma V^{T}$. $U$ and $V$ have the property that $U^{T} U=I, V^{T} V=V V^{T}=I$, and $\Sigma=$ $\operatorname{diag}\left(s_{1}, s_{2}, \ldots, s_{2 n+m}\right), s_{1} \geq s_{2} \geq \ldots s_{2 n+m}$ being the singular values of $A$. Thus, the given set of equations can be reduced to

$$
U \Sigma V^{T} \mathbf{x}=\mathbf{d}
$$

and hence,

$$
\mathbf{x}=V \Sigma^{-1} U^{T} \mathbf{d} .
$$

It can be shown that this gives a least-squares solution to the set of equations (e.g., Golub and Loan 1996). Since the equations have already been normalised by the errors, the standard error on any component $x_{i}$ of vector $\mathbf{x}$ is given by

$$
e_{i}^{2}=\sum_{i=k}^{2 n+m} \frac{v_{i k}^{2}}{s_{k}^{2}}
$$

where $v_{i k}$ are elements of vector $V$.

Smoothing is implemented by replacing the integrals in Eq. (130) by a sum over $M$ uniformly spaced points and adding the corresponding equations to the system of equations to be solved. Thus, the following terms are added:

$$
\frac{\alpha}{\sqrt{M}} q\left(r_{j}\right)\left(\frac{\mathrm{d}^{2}}{\mathrm{~d} r^{2}} \frac{\delta c^{2}}{c^{2}}\right)_{r=r_{j}}=\frac{\alpha}{\sqrt{M}} q\left(r_{j}\right) \sum_{i}^{n} b_{i} \frac{\mathrm{d}^{2}}{\mathrm{~d} r^{2}} \phi_{i}\left(r_{j}\right)=0,
$$

for $i=1 \ldots n$ and $j=1 \ldots M$; and

$$
\frac{\alpha}{\sqrt{M}} q\left(r_{j}\right)\left(\frac{\mathrm{d}^{2}}{\mathrm{~d} r^{2}} \frac{\delta \rho}{\rho}\right)_{r=r_{j}}=\frac{\alpha}{\sqrt{M}} q\left(r_{j}\right) \sum_{i}^{n} c_{i} \frac{\mathrm{d}^{2}}{\mathrm{~d} r^{2}} \phi_{i}\left(r_{j}\right)=0
$$

for $i=n+1 \ldots 2 n$ and $j=M+1 \ldots 2 M$. The function $q(r)$ in the above equation allows us to smooth $\delta c^{2} / c^{2}$ and $\delta \rho / \rho$ preferentially in any given part of the Sun or the star. Thus, there are now $N^{\prime}=N+2 M$ equations in $k=2 n+m$ unknowns which can be solved using SVD.

The 'art' of doing an inversion lies in choosing the correct parameters. There are at least three parameters that need to be specified to do an RLS inversion: (a) the number of basis functions in frequency used to define the surface term, (b) the number of basis functions in radius to describe the two functions, and (c) the regularisation or smoothing parameter $\alpha$. We have the option of increasing the number of parameters by having different numbers of basis functions the two unknown functions (e.g., $c^{2}$ and $\rho$ ) and/or different values of $\alpha$ for the two functions. How these parameters can be determined have been described by Basu and Thompson (1996) and Basu (2014), a brief outline is given here. 
The number of basis functions needed depends on the type of basis functions used. It is most advantageous to have well localised basis functions; this ensure that the solution at one radius is not correlated much with the solution at other radii. Antia and Basu (1994b) and Basu and Thompson (1996) used cubic B-splines (de Boor 2001). Unlike normal cubic splines, these functions are localised. They also have continuous first and second derivatives making it easy to apply smoothing. The positions where B-splines are defined are known as knots and each spline is defined over five knots. $m+2$ knots need to be defined for $m$ basis functions. For inversions of solar frequencies, knots in frequency are usually kept equidistant in frequency. Knots in $r$ are defined so that they are equidistant in the acoustic depth $\tau$. This takes into account the fact that modes spend more time in regions of lower sound speed making those regions easier to resolve.

It is usual to determine the number of frequency knots first. This is done by fixing the number of $r$ knots to a reasonably large value and then examining the residuals left when the number of frequency-knots is changed; the aim is to eliminate structure in the residuals when plotted against frequency. To determine the number of knots in $r$, one can use a combination of the reduced $\chi^{2}$ obtained for reasonable smoothing, as well as the condition number (the ratio of the largest to smallest singular value) of the matrix $A$ in Eq. (131). The two quantities have different behaviours when plotted as a function of the number of knots. There is a sharp decrease in $\chi_{v}^{2}$ as the number of $r$ knots is increased. The number of knots selected should lie after the decrease, where the curve flattens out. The behaviour of the condition number is the opposite, and there is a steep rise beyond a certain number of knots. Experience shows that it is best to select the number of knots from the part of the curve just after the steep jump in the condition number. This position does depend on the smoothing, and hence the selection of the number of knots and the smoothing parameter have to be done in an iterative manner.

The smoothing parameter $\alpha$ is usually determined by inspecting the so-called Lcurve (Hansen 1992) that shows the balance between how smooth the solution is and how large the residuals are by plotting the smoothing constraint $\|L\|^{2}$ (Eq. 130) as a function of the $\chi^{2}$ per degree of freedom. Increasing the smoothing parameter increases the mismatch between the data and the solution, i.e., increases $\chi^{2}$, while decreasing the oscillations in the solution (i.e., lowering $\|L\|^{2}$ ). Decreasing the smoothing parameter reduces $\chi^{2}$, but increases $\|L\|^{2}$. The optimum value of smoothing should lie somewhere in between. The smoothing parameter not only influences how smooth a solution is, it also affects the uncertainties in the solution caused by uncertainties in the data. The higher the smoothing, the lower the uncertainties.

The inversion parameters not only depend on the type of basis function, but also on the number of modes available, their uncertainties, and the distribution of the lower turning points of those modes. Thus one cannot use the same inversion parameters if the mode-set changes.

\subsubsection{Optimally localised averages}

The method of optically localised averages was originally developed for application in geophysics (Backus and Gilbert 1968, 1970). Gough (1985) described how it could be used for solar data. In the following discussion, we will assume that we want to determine the sound-speed differences by inverting Eq. (122). 
OLA inversions involve determining coefficients $c_{i}$ such that the sum

$$
\mathcal{K}\left(r_{0}, r\right)=\sum_{i} c_{i}\left(r_{0}\right) K_{c^{2}, \rho}^{i}(r)
$$

is well localised around $r=r_{0}$. If $\int \mathcal{K}\left(r_{0}, r\right) \mathrm{d} r=1$ then

$$
\left\langle\frac{\delta c^{2}}{c^{2}}\right\rangle\left(r_{0}\right)=\sum_{i} c_{i}\left(r_{0}\right) \frac{\delta \omega_{i}}{\omega_{i}}
$$

is the inversion result at radius $r_{0}$ as long as

$$
\mathcal{C}\left(r_{0}, r\right)=\sum_{i} c_{i}\left(r_{0}\right) K_{\rho, c^{2}}^{i}
$$

is small, and the surface term contribution

$$
\mathcal{F}=\sum_{i} c_{i}\left(r_{0}\right) \frac{F_{\text {surf }}\left(\omega_{i}\right)}{E_{i}}
$$

is small as well. As is clear, the error in the solution will be $e^{2}\left(r_{0}\right)=\sum_{i} c_{i}^{2}\left(r_{0}\right) \sigma_{i}^{2}$, where $\sigma_{i}$ is the error associated with the frequency of mode $i$.

$\mathcal{K}\left(r_{0}, r\right)$ is the "averaging kernel" or "resolution kernel" at $r=r_{0}$. The width of the resolution kernel is a measure of the resolution of the inversions since the kernels represent the region over which the underlying function is averaged. $\mathcal{C}\left(r_{0}, r\right)$ is the "cross-term kernel" and measures the contribution of the second variable (in this case density) on the inversion results. The aim of OLA inversions is to obtain the narrowest possible averaging kernels that the data allow, keeping the uncertainty in the solution to acceptable levels, at the same time minimising the effect of the cross-term kernel on the solution.

There are two widely used implementations of OLA. The first is the original one, which helioseismology community often calls the Multiplicative Optimally Localised Averages, or MOLA. MOLA is computation-intensive and requires a large matrix to be set up and inverted at reach target radius $r_{0}$. A less computationally intensive implementation, the Subtractive Optimally Localised Averages (SOLA) was introduced by Pijpers and Thompson (1992). SOLA requires just one matrix inversion.

\section{Implementing OLA}

The inversion coefficients for MOLA are determined by minimising

$$
\int\left(\sum_{i} c_{i} K_{c^{2}, \rho}^{i}\right)^{2} J\left(r_{0}, r\right) \mathrm{d} r+\beta \int\left(\sum_{i} K_{\rho, c^{2}}^{i}\right)^{2} \mathrm{~d} r+\mu \sum_{i, j} c_{i} c_{j} E_{i j}
$$

where, $J=\left(r-r_{0}\right)^{2}$ (often $J$ is defined as $12\left(r-r_{0}\right)^{2}$ ) and $E_{i j}$ are elements of the error-covariance matrix. If the uncertainties in the mode frequencies are uncorrelated, 
then $E_{i j}=\sigma_{i} \delta_{i j}$. This implementation is called the 'Multiplicative' OLA because the function $J$ multiplies the averaging kernel in the first term of Eq. (141). The parameter $\beta$ ensures that the cross-term kernel is small, and $\mu$ ensures that the error in the solution is small. The constraint of unimodularity of the averaging kernel $\mathcal{K}$ is applied through a Lagrange multiplier. The surface term is expressed as

$$
F_{\text {surf }}(\omega)=\sum_{j=1}^{\Lambda} a_{j} \Psi_{j}(\omega)
$$

$\Psi_{j}(\omega)$ being suitable basis functions and $\Lambda$ is the number of basis functions. Equation (141) is minimised subject to the condition that the contribution of $F_{\text {surf }}(\omega)$ to the solution is small. This is done by adding constraints of the form

$$
\sum_{i} c_{i} \frac{\Psi_{j}\left(\omega_{i}\right)}{E_{i}}=0, \quad j=1, \ldots, \Lambda
$$

SOLA proceeds through minimising

$$
\int\left(\sum_{i} c_{i} K_{c^{2}, \rho}^{i}-\mathcal{T}\right)^{2} \mathrm{~d} r+\beta \int\left(\sum_{i} K_{\rho, c^{2}}^{i}\right)^{2} \mathrm{~d} r+\mu \sum_{i, j} c_{i} c_{j} E_{i j}
$$

where $\mathcal{T}$ is the target averaging kernel. The difference between the averaging and target kernels in the first term of Eq. (144) is the reason why this implementation is called 'Subtractive' OLA. The condition of unimodularity for the averaging kernels and the surface constraints have to be applied as before.

The choice of the target kernel is often dictated by what the purpose of the inversion is. It is usual to use a Gaussian, as was done by Pijpers and Thompson (1992). They used

$$
\mathcal{T}\left(r_{0}, r\right)=A \exp \left(-\left[\frac{r-r_{0}}{\Delta\left(r_{0}\right)}\right]^{2}\right)
$$

where $A$ is a normalisation factor that ensures that $\int \mathcal{T} \mathrm{d} r=1$. However, this form has the disadvantage that for small $r_{0}, \mathcal{T}$ may not be equal to 0 at $r=0$ where the kernels $K_{c^{2}, \rho}$ and $K_{\rho, c^{2}}$ are zero. This leads to a forced mismatch between the target and the resultant averaging kernel. One way out is to force the target kernels to go to zero at $r=0$ using a modified Gaussian such as

$$
\mathcal{T}\left(r_{0}, r\right)=\operatorname{Ar} \exp \left(-\left[\frac{r-r_{0}}{\Delta\left(r_{0}\right)}+\frac{\Delta\left(r_{0}\right)}{2 r_{0}}\right]^{2}\right),
$$

where again, $A$ is the normalisation factor that ensures that the target is unimodular.

The width of the averaging kernel at a given target radius $r_{0}$ depends on the amount of information present for that radius, the widths are usually smaller towards the surface than towards the core. This is a reflection of the fact that (for p modes at least) the 
amplitudes of the kernels are larger towards the surface. Thus the target kernels need to be defined such that they have a variable width. The usual practice is to define the width $\Delta_{f}$ at a fiducial target radius $r_{0}=r_{f}$ and specify the widths at other locations as $\Delta\left(r_{0}\right)=\Delta_{f} c\left(r_{0}\right) / c\left(r_{f}\right)$, where $c$ is the speed of sound. This inverse variation of the width with sound speed reflects the ability of modes to resolve stellar structure (see Thompson 1993).

The equations that result from the minimisation of Eqs. (141) and (144) and applying the constraints can be written as a set of linear equations of the form

$$
A \mathbf{c}=\mathbf{v}
$$

For $M$ observed modes the matrix elements $A_{i j}$ for SOLA inversions are given by

$$
A_{i j}= \begin{cases}\int K_{c^{2}}^{i} K_{c^{2}}^{j} \mathrm{~d} r+\beta \int K_{\rho}^{i} K_{\rho}^{j} \mathrm{~d} r+\mu E_{i j} & (i, j \leq M) \\ \int K_{c^{2}}^{i} \mathrm{~d} r & (i \leq M, j=M+1) \\ \int K_{c^{2}}^{j} \mathrm{~d} r & (j \leq M, i=M+1) \\ 0 & (i=j=M+1) \\ \Psi_{j}\left(\omega_{i}\right) / E_{i} & (i \leq M, M+1<j \leq M+1+\Lambda) \\ \Psi_{i}\left(\omega_{j}\right) / E_{j} & (M+1<i \leq M+1+\Lambda, j \leq M) \\ 0 & \text { (otherwise) }\end{cases}
$$

The vectors $\mathbf{c}$ and $\mathbf{v}$ have the form

$$
\mathbf{c}=\left(\begin{array}{c}
c_{1} \\
c_{2} \\
\cdot \\
\cdot \\
\cdot \\
c_{M} \\
\lambda \\
0 \\
\cdot \\
\cdot \\
0
\end{array}\right), \quad \text { and } \quad \mathbf{v}=\left(\begin{array}{c}
\int K_{c^{2}}^{1} \mathcal{T} \mathrm{d} r \\
\int K_{c^{2}}^{2} \mathcal{T} \mathrm{d} r \\
\cdot \\
\cdot \\
\cdot \\
\int K_{c^{2}}^{M} \mathcal{T} \mathrm{d} r \\
1 \\
0 \\
\cdot \\
\cdot \\
0
\end{array}\right)
$$

For MOLA, the elements of $A$ for $i, j \leq M$ change, and are given by

$$
A_{i j}=\left(1-r_{0}\right)^{2} \int K_{c^{2}}^{i} K_{c^{2}}^{j} \mathrm{~d} r+\beta \int K_{\rho}^{i} K_{\rho}^{j} \mathrm{~d} r+\mu E_{i j} .
$$

And as far as the vector $\mathbf{v}$ is concerned, the $(M+1)$ th element for MOLA is the same as that for SOLA, but all other elements are equal to 0 . The column vector $\mathbf{c}$ is identical 
Fig. 13 A sample of averaging kernels for sound-speed inversions obtained with the SOLA method. The averaging kernels were obtained for inversions of the frequency differences between Model S and the solar dataset BiSON-13 of Basu et al. (2009). The results are for the inversions reported in that paper

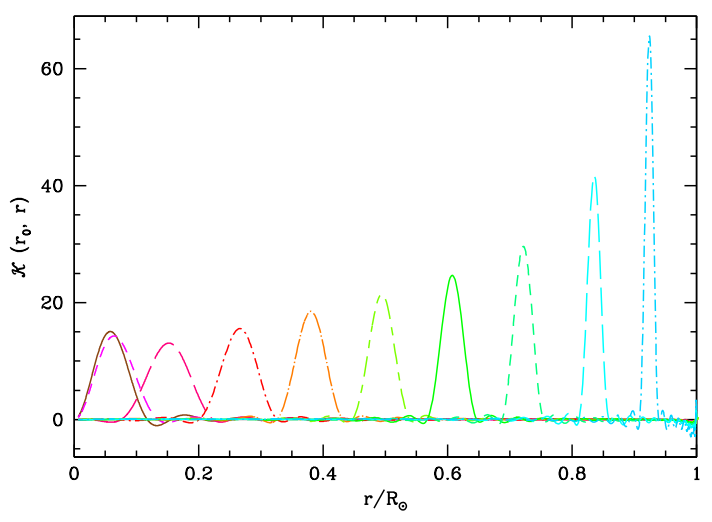

in both cases. Note that in the case of MOLA, $A$ is a function of the target radius $r_{0}$. As a result, the matrix has to be set up and inverted at every point where we need a result. This makes MOLA very computationally expensive.

Choosing inversion parameters for OLA is a bit more difficult than that for RLS. For MOLA inversions we need to determine at least three parameters-the number of surface terms $\Lambda$, the error suppression parameter $\mu$ and the cross-term suppression parameter $\beta$. A fourth parameter, the width of the target averaging kernels $\Delta\left(r_{f}\right)$ at a fiducial radius of $r_{f}$, is needed for SOLA. Rabello-Soares et al. (1999) and Basu (2014) give extensive discussions on how parameters can be selected. In Fig. 13 we show a few sample averaging kernels from the inversions reported in Basu et al. (2009).

As in the case of RLS inversions, the surface term is the first parameter to be determined. It is done by fitting different numbers of basis functions $\Psi$ to the frequency differences to be inverted. The fit is done to the scaled differences. The number of functions is increases till there is no large-scale structure discernible when the residuals are plotted against frequency.

In the case of SOLA, the width of the target averaging kernel is such that the mismatch between the obtained and target averaging kernel is minimised. The mismatch can be defined as:

$$
\chi\left(r_{0}\right)=\int\left[\mathcal{K}\left(r_{0}, r\right)-\mathcal{T}\left(r_{0}, r\right)\right]^{2} \mathrm{~d} r .
$$

The target kernels as defined by either Eqs. (145) or (146) are all positive. However, if the selected target width is too small, the resultant averaging kernels have negative side-bands. Too narrow averaging kernels also result high uncertainties.

In the case of MOLA, the error-parameter $\mu$ and the cross-term parameter $\beta$ determine the averaging kernel. While no mismatch can be defined there, one can try to minimise the negative sidebands by ensuring that the quantity

$$
\chi^{\prime}\left(r_{0}\right)=\int_{0}^{r_{A}} \mathcal{K}^{2}\left(r_{0}, r\right) \mathrm{d} r+\int_{r_{B}}^{1} \mathcal{K}^{2}\left(r_{0}, r\right) \mathrm{d} r
$$

is small. Here, $r_{A}$ and $r_{B}$ are defined in such a way that the averaging kernel $\mathcal{K}$ has its maximum at $\left(r_{A}+r_{B}\right) / 2$ and its full width at half maximum is $\left(r_{B}-r_{A}\right) / 2$. 
In practice, small negative side lobes can be an advantage. Since the solutions at all radii are obtained from the same set of data, the error in the inversion result at one radius is correlated with that at another. The error correlation between solutions at radii $r_{1}$ and $r_{2}$ are given by

$$
E\left(r_{1}, r_{2}\right)=\frac{\sum c_{i}\left(r_{1}\right) c_{i}\left(r_{2}\right) \sigma_{i}^{2}}{\left[\sum c_{i}^{2}\left(r_{1}\right) \sigma_{i}^{2}\right]^{1 / 2}\left[\sum c_{i}^{2}\left(r_{2}\right) \sigma_{i}^{2}\right]^{1 / 2}}
$$

The error correlation has values between \pm 1 . A value of +1 implies complete correlation while value of -1 implies complete anti-correlation. Correlated errors can introduce features into the solution on the scale of the order of the correlation function width (Howe and Thompson 1996). While wide averaging kernels reduce $\chi\left(r_{0}\right)$ and $\chi^{\prime}\left(r_{0}\right)$ and also reduce uncertainties in the results, they can increase error correlations and give rise to spurious features in the inversions.

It should be noted that it is almost impossible to reduce error correlations for density inversions. The conservation of mass condition forces the solution in one part of the star to be sensitive to the solution at other parts of the star. There is an anti-correlation of errors between the core (where density is the highest) and the outer layers (where density is the lowest), and the cross-over occurs around the radius at which $r^{3} \rho$ has the largest value.

For fixed values of the cross-term suppression parameter $\beta$, and the errorsuppression parameter $\mu$, changing the width of the averaging kernel changes the cross-term kernels, and hence the contribution of the second function on the inversion results of the first function. The contribution of the cross-term kernels can be gauged using the quantity

$$
C\left(r_{0}\right)=\sqrt{\int \mathcal{C}^{2}\left(r_{0}, r\right) \mathrm{d} r} .
$$

We need to aim for small $C\left(r_{0}\right)$ in order to get a good inversion. The requirement that the error-correlation be small acts against the other requirements.

There are two other parameters that need to be determined, the error-suppression parameter $\mu$ and the cross term suppression parameter $\beta$. Increasing $\mu$ decreases the error correlation $E\left(r_{1}, r_{0}\right)$ and the uncertainty in the solution $e\left(r_{0}\right)$, but increases $\chi\left(r_{0}\right)$ the mismatch between the target kernels and the averaging kernel. The influence of the cross term as measured by $C\left(r_{0}\right)$ also increases. Increasing $\beta$ increases $E\left(r_{1}, r_{0}\right)$, $e\left(r_{0}\right)$, and $\chi\left(r_{0}\right)$, but decreases $C\left(r_{0}\right)$. Given that the cross term parameter reduces systematic errors while the error-suppression parameter reduces random errors, it is usual to try and reduce $C\left(r_{0}\right)$ even if that results in a somewhat larger uncertainty in the solution.

Given the complexity of the parameter finding process, it is advisable to start the inversion process by first to examining the behaviour of the solutions obtained by inverting frequency differences between two models using the observed modeset. 
Fig. 14 Relative density differences between the Sun and model BP04 of Bahcall et al. (2005a) obtained using both RLS and SOLA techniques. The results marked 'All' were obtained using the modeset GOLF1low (see text) while the results marked 'Weeded' where obtained when the $\ell=0, n=3$ and $\ell=3, n=5$ modes were removed from the set. Note that RLS results remain the same, but SOLA results change drastically. The inversion results are from Basu et al. (2009)

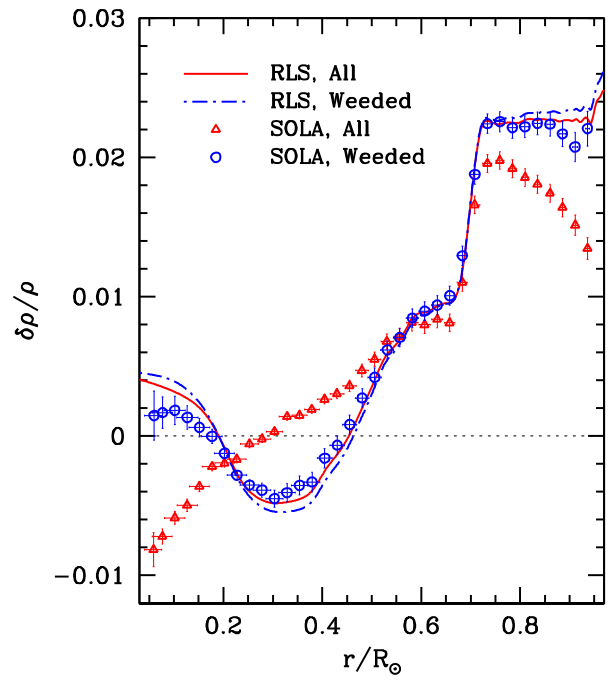

\subsubsection{Ensuring reliable inversions}

Since inversion results do depend on the parameters chosen, the most reliable results are obtained when RLS and OLA results agree since the two methods are complementary (Sekii 1997).

OLA inversions are notoriously prone to systematic errors caused by outliers, while RLS results are relatively insensitive. Unless both types of inversions are done, one often cannot detect the error due to bad data points. An example of this is shown in Fig. 14. The figure shows the inverted density difference between the Sun and model BP04 of Bahcall et al. (2005a). The modeset was a combination of $\ell=0,1,2$, and 3 modes with frequencies less than $1800 \mu \mathrm{Hz}$ obtained by GOLF as listed in Bertello et al. (2000) supplemented with modes from the first year of MDI (Schou et al. 1998b). This was the so-called 'GOLF1low' modeset of Basu et al. (2009). RLS inversions show extremely large residuals for the $\ell=0, n=3$ and $\ell=0, n=5$ modes. Almost identical results were obtained with RLS inversions whether or not these two modes are included in the modeset that is inverted. SOLA inversion results on the other hand changed drastically, especially at very low radii. This discrepancy would not have been noticed had both RLS and SOLA inversions had not been performed, and erroneous results about the solar core would have been drawn.

The inversion results are also only as correct as the kernels used for the inversion. It used to be customary to determine the difference between the density profiles of the Sun and models using kernels of $(\rho, Y)$ that were derived from kernels of $\left(\rho, \Gamma_{1}\right)$ using the expansion given in Eq. (119). Such a transformation can be done if one assumes that the equation of state of the model is the same as that for the Sun. Basu and Christensen-Dalsgaard (1997) realised that this could give rise to systematic errors in the results. In the early days of helioseismology, the systematic errors were smaller than the uncertainties caused by data errors, but as uncertainties in the frequencies have become smaller, these systematic errors have become significant. An example of this 
Fig. 15 The relative difference in the density between the Sun and reference model $\mathrm{S}$

(Christensen-Dalsgaard et al. 1996) obtained by inverting the "Best set" of Basu et al. (1997). Two results are shown, one obtained using the $(\rho, Y)$ kernel combination, the other using the $\left(\rho, \Gamma_{1}\right)$ kernel combination.

Note the striking differences between the two results, especially in the core

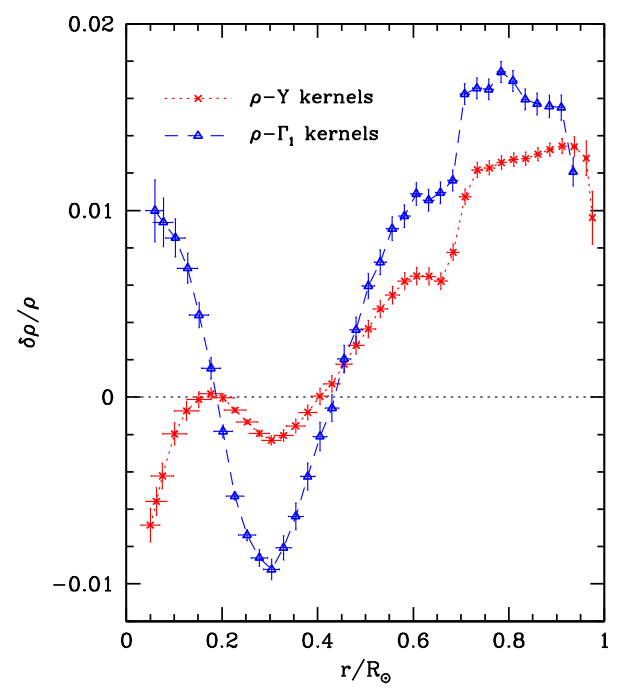

effect is shown in Fig. 15 where we show the frequency difference between the Sun and Model S of Christensen-Dalsgaard et al. (1996). The inversions were performed using both $\left(\rho, \Gamma_{1}\right)$ and $(\rho, Y)$ kernels. Note that the results differ significantly in the core. One result (the one with $\rho, \Gamma_{1}$ ) implies that the solar core is denser than that Model $\mathbf{S}$, the other implies the opposite. Of these the results with $\rho, \Gamma_{1}$ kernels are more reliable given that no assumption about any of the microphysics of the Sun went into its derivation.

$(\rho, Y)$ and $(u, Y)$ kernels had been used extensively in early inversion because $Y$ kernels are non-zero only in the helium ionisation zone. This made it very easy to suppress the cross-term while keeping the error low even with the early, limited data sets. The price paid was the systematic error caused by differences between the equation of state of solar material and that used to construct solar models. Basu and Christensen-Dalsgaard (1997) showed that it is still possible to use $(\rho, Y)$ and $(u, Y)$ kernels without introducing errors by adding another term in Eq. (119) that accounts for the intrinsic differences between the equations of state. Thus

$$
\begin{aligned}
\frac{\delta \Gamma_{1}}{\Gamma_{1}} & =\left(\frac{\delta \Gamma_{1}}{\Gamma_{1}}\right)_{\mathrm{int}}+\left(\frac{\partial \ln \Gamma_{1}}{\partial \ln P}\right)_{\rho, Y} \frac{\delta P}{P}+\left(\frac{\partial \ln \Gamma_{1}}{\partial \ln \rho}\right)_{P, Y} \frac{\delta \rho}{\rho}+\left(\frac{\partial \ln \Gamma_{1}}{\partial Y}\right)_{\rho, P} \delta Y \\
& =\left(\frac{\delta \Gamma_{1}}{\Gamma_{1}}\right)_{\mathrm{int}}+\Gamma_{1, P} \frac{\delta P}{P}+\Gamma_{1, \rho} \frac{\delta \rho}{\rho}+\Gamma_{1, Y} \delta Y
\end{aligned}
$$

where $\left(\delta \Gamma_{1} / \Gamma_{1}\right)$ int is the intrinsic difference between the equations of state. This term is a second cross term, and if its influence is minimised, the systematic errors due to the uncertainties in the equation of state become negligibly small. There is a price to pay though, the uncertainty in the solution becomes as large as those obtained when $\left(\rho, \Gamma_{1}\right)$ kernels are used for density inversions, eliminating any advantage that using $Y$ kernels may have given. 

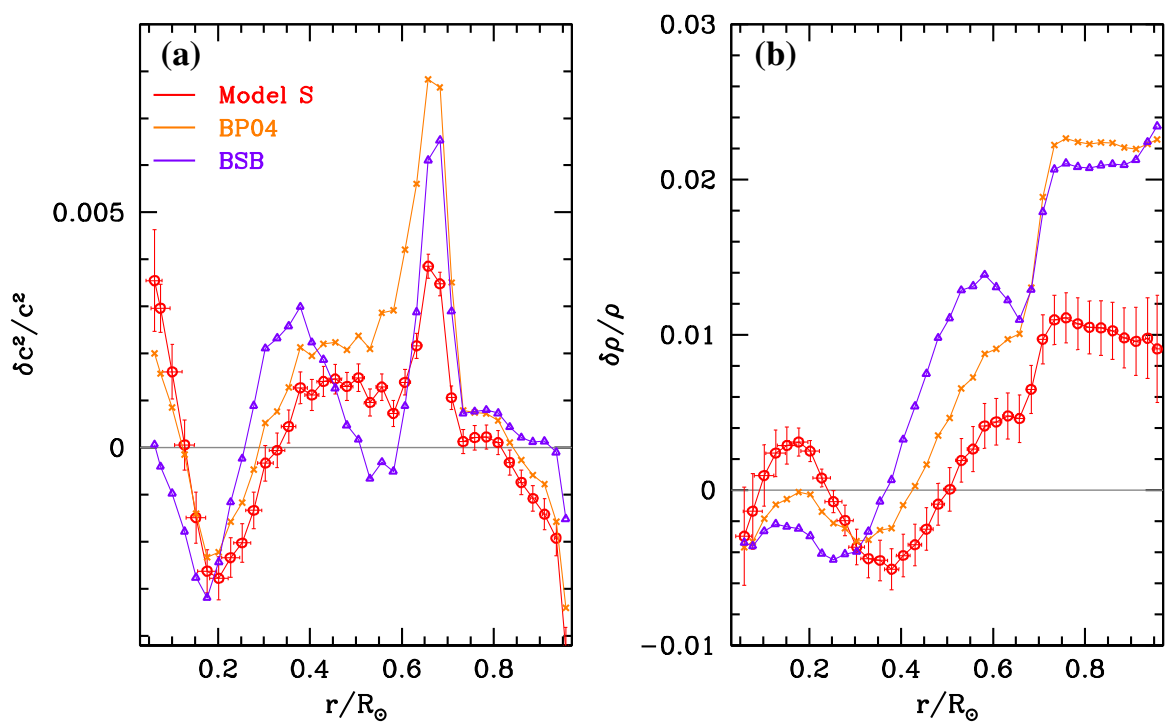

Fig. 16 The relative difference in the squared sound speed and density between the Sun and three solar models used earlier in Fig. 7. The difference are in the sense (Sun-Model). The results are from Basu et al. (2009) obtained using their BiSON-13 data set. In the figure the vertical error-bars show $3 \sigma$ uncertainties in the results caused by errors in the input frequencies. The horizontal error-bars are a measure of the resolution of the inversions. They show the distance between the first and third quartile points of the resolution kernels. Error-bars are only shown for one model for the sake of clarity, they are the same for all models

Although structure inversions rely on a linearisation of the oscillation equations around a reference model, given the quality of current solar models, this does not appear to add any errors. Basu et al. (2000a) examined the influence of the reference model on the estimated sound-speed, density and $\Gamma_{1}$ profile of the Sun. They found that the effect is negligible, much smaller than the uncertainties caused by data errors, when one uses modern solar models.

\section{Results from structure inversions}

\subsection{Solar structure and the solar neutrino problem}

Inversions of solar oscillation frequencies have allowed us to determine the solar sound-speed, density and $\Gamma_{1}$ profiles (see e.g., Christensen-Dalsgaard et al. 1989b; Dziembowski et al. 1990; Däppen et al. 1991; Antia and Basu 1994b; Gough et al. 1996; Basu et al. 1997, 2000a, b, 2009; Turck-Chièze et al. 1997). Inversions using solar p mode data usually reveal the structure of the Sun from $0.06 R_{\odot}$ to $0.96 R_{\odot}$ (e.g., Basu et al. 2009). The lack of reliable high-degree modes makes probing the nearsurface regions more difficult. The inner bound is set by the number of low-degree modes.

The inversion results show that modern standard solar models agree quite well with the Sun, certainly by usual astronomical standards. In Fig. 16, we show the 
relative sound-speed and density differences between the Sun and three standard solar models. Note that the sound-speed difference is fractions of a percent, while the density difference is about $2 \%$ at the maximum. There are however, statistically significant differences in some regions. In the case of sound speed, we find a large difference just below the base of the convection zone. The peak in the sound-speed difference has been attributed to the steep gradient in the helium and heavy-element abundances just below the convection zone of the models. All models shown in the figure include diffusion. Diffusion increases the abundance of helium and metals just below the base of the convection zone, which in turn decreases the sound speed in the models (since $c^{2} \propto 1 / \mu, \mu$ being the mean molecular weight). This localised difference can be reduced if some mixing is included below the base of the convection zone (e.g., Richard et al. 1996; Basu et al. 2000a; Brun et al. 2002). There are indeed some helioseismic investigations that suggest that the increase in $Y$ and $Z$ below the base of the convection zone is not as steep as that predicted by standard solar models (Kosovichev 1996; Antia and Chitre 1998). Inversions for solar rotation (see e.g., Thompson et al. 1996; Schou et al. 1998a, and references therein. More in Sect. 8.) show that there is a strong shear layer at the base of the convection zone that is usually referred to as the "tachocline". This shear layer could easily cause mixing in the radiative-region just below the convection zone, thereby smoothing out abundance gradients (e.g., Zahn 1992). Models that include rotationally-induced mixing do indeed have a smoothed out profile (see e.g., Richard et al. 1996). The dip in the sound-speed difference around $0.2 R_{\odot}$ has not been completely explained yet, though it is often interpreted as being caused by extra mixing in the early life of the Sun that is not present in the models (Gough et al. 1996). Density differences are more difficult to interpret since the conservation of mass condition implies that a large difference in one part of the Sun has to be compensated by an opposite difference elsewhere.

The extremely small difference between the structure of standard solar models and the Sun gave the first hints to the solution of the so-called solar neutrino problem. Solar neutrino measurements started with a Chlorine based detector (Davis 1964, 1994). Other early experiments included the water Cerenkov experiments Kamiokande (Totsuka 1992; Suzuki 1995) and Super Kamiokande (Suzuki 1991) and gallium experiments GALLEX (Anselmann et al. 1995) and SAGE (Abdurashitov et al. 1994). The solar neutrino problem arose in the 1970s when it was found that the observed flux of neutrinos from the Sun using the Chlorine-based detector was only about a third of the neutrino flux predicted by models. The problem was confirmed by later observations using other detectors based on water and gallium. The small difference in structure between solar models and the Sun led many helioseismologists to conclude that the solution to the solar neutrino problem must lie with the standard model of particle physics which postulates mass-less neutrinos. Early claims were based purely on frequency comparisons (Elsworth et al. 1990a) and comparisons of small separations (Elsworth et al. 1995a); later claims were based in inversion results (e.g., Bahcall et al. 1997; Takata and Shibahashi 1998; Watanabe and Shibahashi 2001, etc.). In fact if a non-standard solar model is constructed so that the neutrino constraints from the Chlorine experiment are satisfied, the sound-speed difference between the model and the Sun at the core would be about $10 \%$ (Bahcall et al. 1998), which is much larger than what is seen for standard solar models. The solar neutrino problem had a sec- 
ond component, it was found that the number of neutrinos detected by the Chlorine detector was not consistent with those detected by the water-based detectors, which in turn were inconsistent with the gallium-based measurements. Antia and Chitre (1997) showed that it was not possible to construct a solar model that satisfied all three solar neutrino constrains (i.e., those given by the Chlorine, water and Gallium experiments) simultaneously even if some of the solar constraints were relaxed. Hata et al. (1994), Haxton (1995), Castellani et al. (1997) and Heeger and Robertson (1996) made similar inferences using the observed data alone, without involving solar models. Thus it was evident that the solution to the solar neutrino problem did not lie in deficiencies of modelling the Sun, but in the assumption regarding properties of neutrinos. The particle-physics solution to the solar neutrino problem has since been confirmed by results from the Sudbury Neutrino Observatory (Ahmad et al. 2002), and the solar neutrino problem is one of the few examples of fully solved problems in astrophysics.

\subsection{Some properties of the solar interior}

While inversions have allowed us to determine solar structure in general, the finite resolution of the inversions has often meant that specialised techniques have had to be applied to determine some of the finer details about the solar interior, these include determining the exact position of the base of the solar convection zone $\left(r_{b}\right)$, the amount of overshoot below the solar convection zone as well as the amount of helium in the convection zone $\left(Y_{S}\right)$.

\subsubsection{The base of the convection zone}

The base of the convection zone is defined as the layer where the temperature gradient changes from being adiabatic to radiative. This definition means that $r_{b}$ for standard solar models is defined unambiguously. For solar models with overshoot below the base of the convection zone, this definition results in some ambiguity. When overshoot is modelled as a well-mixed region as well as a region which is adiabatically stratified, $r_{b}$ defines the edge of the overshooting region. In models where overshooting is merely a well mixed region, $r_{b}$ is the true base of the convection zone. The abrupt change in the temperature gradient, from adiabatic to radiative, at $r_{b}$ results in a large change in the sound-speed difference between two models that have different values of $r_{b}$ and this is illustrated in Fig. 17. The change in sound speed can be used to determine the position of the convection-zone base of the Sun.

Ulrich and Rhodes (1977) and Rhodes et al. (1977) compared early helioseismic data with models to show that the Sun has a deeper convection zone than the models of that era, and that the solar $r_{b}$ was between $0.62 R_{\odot}$ and $0.75 R_{\odot}$. Berthomieu et al. (1980) estimated the depth of the solar convection zone to be $200 \mathrm{Mm}$. Precise estimates of solar $r_{b}$ had to wait for better data sets.

Christensen-Dalsgaard et al. (1991) used asymptotic sound-speed inversion results to determine the dimensionless sound-speed gradient $W(r)$ given by 


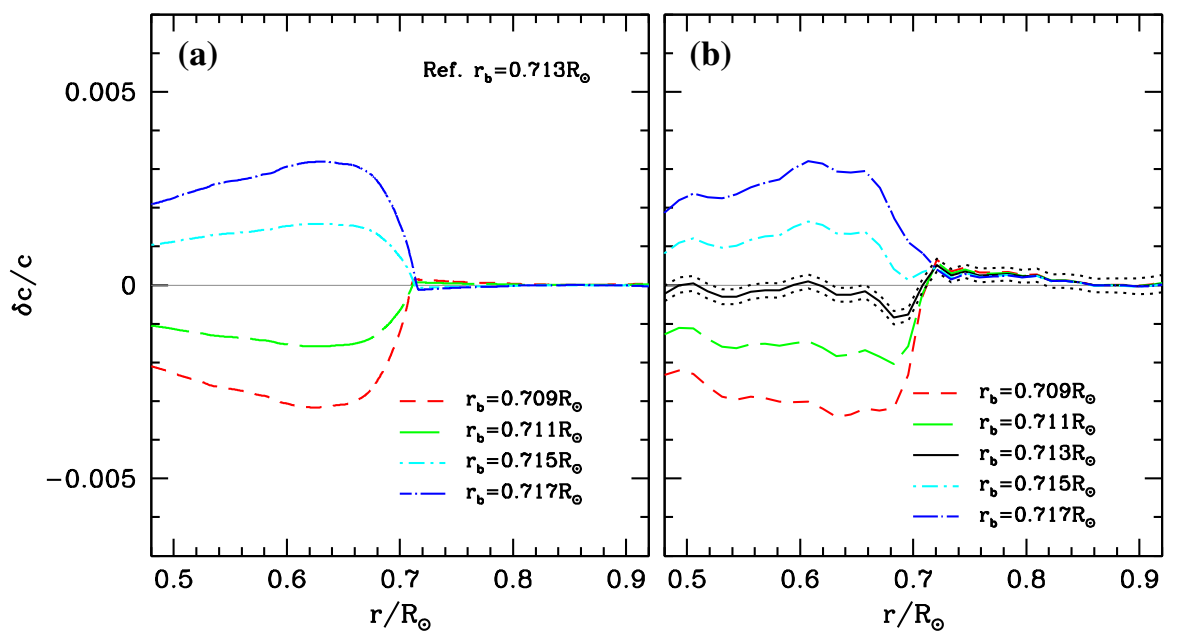

Fig. 17 a The relative sound-speed difference between a solar envelope model with a convection zone base $r_{b}$ at $0.713 R_{\odot}$ and other models with different values of $r_{b}$. All other physics inputs to the models are identical. b The sound-speed difference between the Sun and the different models in a. The solar sound-speed results are from Basu et al. (2009). The dotted lines show the $2 \sigma$ uncertainties on the solar results

$$
W(r) \equiv \frac{r^{2}}{G m} \frac{\mathrm{d} c^{2}}{\mathrm{~d} r} .
$$

$W(r)$ is nearly constant, and equal to -0.67 , in the deeper part of the convection zone. It increases abruptly below the convection-zone base. Christensen-Dalsgaard et al. (1991) used the position of this increase to find that the base of the solar convection zone was located at $r_{b}=0.713 \pm 0.001 R_{\odot}$. Kosovichev and Fedorova (1991) obtained similar results. Basu and Antia (1997) did a detailed study of the systematic errors involved in the process. They used the function $H_{1}(w)$ (Eq. 89) obtained between the Sun and models with different values of $r_{b}$ to determine position of the solar convection-zone base. Their results, despite using much better data, was identical to that of Christensen-Dalsgaard et al. (1991). Basu (1998) studied whether or not errors in measurement of the solar radius would have any effect on $r_{b}$ determinations, she found none, and using data from the GONG and MDI projects she obtained $r_{b}=$ $0.7133 \pm 0.0005 R_{\odot}$. Basu and Antia (2004) examined whether the adopted value of the solar heavy-element abundance has any effect on the estimated value of solar $r_{b}$ and found none. Thus the position of the solar convection zone base is known sufficiently precisely to provide a strong constraint that solar models need to satisfy. The question of whether the base of the solar convection zone has any latitudinal dependence has also been studied by different groups. Gough and Kosovichev (1995) inverted for $\delta u / u$ between the Sun and a spherically symmetric model using BBSO data to find that the equator may be somewhat deeper than that at the poles, but stated that the difference did not exceed $0.02 R_{\odot}$. However, neither Monteiro and Thompson (1998) nor Basu and Antia (2001a) found any such latitudinal variation. 


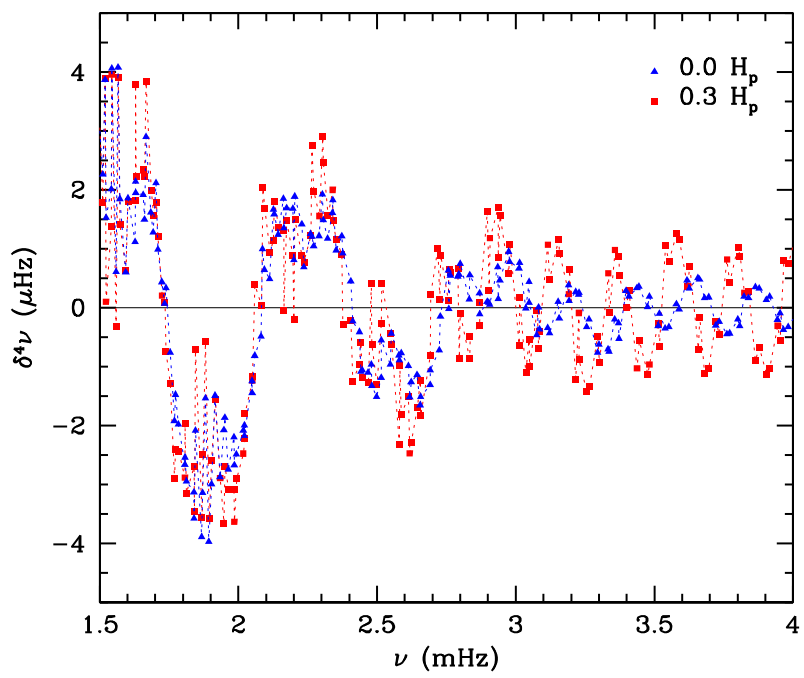

Fig. 18 The signature of the acoustic glitches in two solar models, one without overshoot (blue triangles) and one with an overshoot of $0.3 H_{p}$ (red squares). The signature has been enhanced by taking the fourth differences of the frequencies. Dotted lines joining the points are meant merely to guide the eye, the jaggedness of the lines reflects the $\ell$-dependence of the signature. Note that there are two distinct oscillatory patterns, the larger-wavelength one is the signature of the He II ionisation zone, the smaller wavelength one is from the base of the convection zone. Note that the amplitude of the smaller-wavelength pattern is larger for the model with overshoot

The abrupt change of the temperature gradient at the convection-zone base is an acoustic glitch that leaves its signature on the frequencies [see Sect. 5, Eq. (83)], and this signature can be isolated to study the region. The amplitude of the signal is a function of the size of the glitch. Simple models of convective overshoot model the phenomenon by extending the adiabatic temperature gradient artificially and then changing over to the radiative gradient below the overshoot region. This increases the size of the acoustic glitch and that can be used to determine the extent of overshoot below the solar convection zone, an idea put forward by Gough and Sekii (1993). In Fig. 18, we show this for two solar models. Assuming that this model of overshoot is correct, then overshoot below the solar convection-zone base is very small (e.g., Monteiro et al. 1994; Basu et al. 1994; Basu and Antia 1994; Roxburgh and Vorontsov 1994; Basu 1997), and Basu (1997) put an upper limit of $0.05 H_{p}$ on the amount of overshoot. Of course, such a simple model of overshoot is unlikely to be correct and there have been attempts to modify the change of the temperature gradient to make it more realistic (Christensen-Dalsgaard et al. 1995, however, what is realistic is still a matter of debate (see, e.g., Rempel 2004). Two-dimensional numerical simulations suggest that overshoot could lead to a slightly extended mildly adiabatic temperature gradient beneath the convection zone up to $0.05 H_{p}$ followed by a rapid transition to a strongly sub-adiabatic region (Rogers et al. 2006). There are few 3D simulations that are focused on studying the overshooting region, and there are issues with those (see Canuto 2011, for an account). 


\subsubsection{The convection-zone helium abundance}

Another important property of the Sun is its convection-zone helium abundance $Y_{S}$. Since the Sun is a cool star, $Y_{s}$ cannot be measured spectroscopically. Yet, this is an important parameter that controls solar evolution. The convection-zone helium abundance is expected to be lower than the initial helium abundance $Y_{0}$ of the Sun because of diffusion and gravitational settling, but nevertheless it can constrain models of the current Sun. Helium leaves its signature on solar oscillation frequencies because it changes the mean molecular weight (and hence sound speed), it also contributes an acoustic glitch in the form of the He II ionisation zone (the He I ionisation zone for the Sun overlaps with the $\mathrm{HI}$ ionisation zone and as a result causes large systemic error when determining the helium abundance, and hence it is not used for such studies). It is the second effect that is usually exploited in different ways to determine the solar helium abundance.

The first attempts to determine $Y_{S}$ (Däppen and Gough 1986; Däppen et al. 1988) were handicapped by the lack of high-precision data. More successful attempts were made after the publication of the Libbrecht et al. (1990) frequencies. Däppen et al. (1991) did a full inversion using kernels for $(u, Y)$ to obtain $Y_{s}=0.268 \pm 0.01$; Dziembowski et al. (1991) on the other hand found $Y_{s}=0.234 \pm 0.005$. Kosovichev et al. (1992) found $Y_{s}=0.232 \pm 0.005$; they also investigated the reason for the large variation in the estimated values of $Y_{s}$ and found that a major source of systematic error is the equation of state needed to convert the $\left(c^{2}, \rho\right)$ kernels to the $(u, Y)$ kernels. Kosovichev (1993) used low degree data from the IPHIR instrument (Toutain and Froehlich 1992), and used the $A^{*}-Y$ kernel combination to examine whether lowdegree modes are enough to determine $Y ; A^{*}$ is negligible in the $\mathrm{CZ}$ and hence its contribution to the inversion should be small. He found that $Y_{s}=0.251$ and 0.256 for the two data sets he used. The spread in the values of $Y_{S}$ remained even when better data from GONG and MDI became available. Using reference models constructed with the MHD equation of state Richard et al. (1998) estimated $Y_{S}$ to be $0.248 \pm 0.002$ which using models constructed with the OPAL equation of state Di Mauro et al. (2002) determined it to be $0.2539 \pm 0.0005$.

Performing direct inversions is only one way to determine the solar helium abundance. Vorontsov et al. (1992) by using an asymptotic description of the oscillation equations concluded that $Y_{s}=0.25 \pm 0.01$. Guzik and Cox (1992) did a straightforward frequency comparison to find $Y_{s}=0.24 \pm 0.005$. Antia and Basu (1994a) used a different method altogether-following the idea of Gough (1984b) they used the dimensionless sound speed gradient $W(r)$ (Eq. 156) to determine $Y_{s}$. The height of $W(r)$ in the helium ionisation zone is a function of the helium abundance, unfortunately it also depends on the equation of state (see Fig. 19). They found that $W(r)$ for models constructed with the EFF equation of state did not match observations at all. Using models constructed with MHD equations of state they estimated $Y_{S}$ to be $0.252 \pm 0.003$. Once the OPAL equation of state was made available, Basu and Antia (1995) showed that $Y_{s}$ estimates using calibration method, when either $W(r)$ or $H_{1}(w)$ were calibrated against models of know helium abundance, were less sensitive to equation-of-state effects than full inversions. Using the Libbrecht et al. (1990) data they obtained $Y_{s}=0.246$ using MHD models, and $Y_{s}=0.249$ using OPAL mod- 
Fig. 19 A comparison of the observed dimensionless sound speed gradient of the Sun in the region of the He II ionisation zone (black line) with that of different models constructed with different equations of state and different convection-zone helium abundances. The heavy-element abundance is the same for all models. Note that the height of $W(r)$ depends on the helium abundance for a given equation of state, but for a given helium abundance it depends on the equation of state

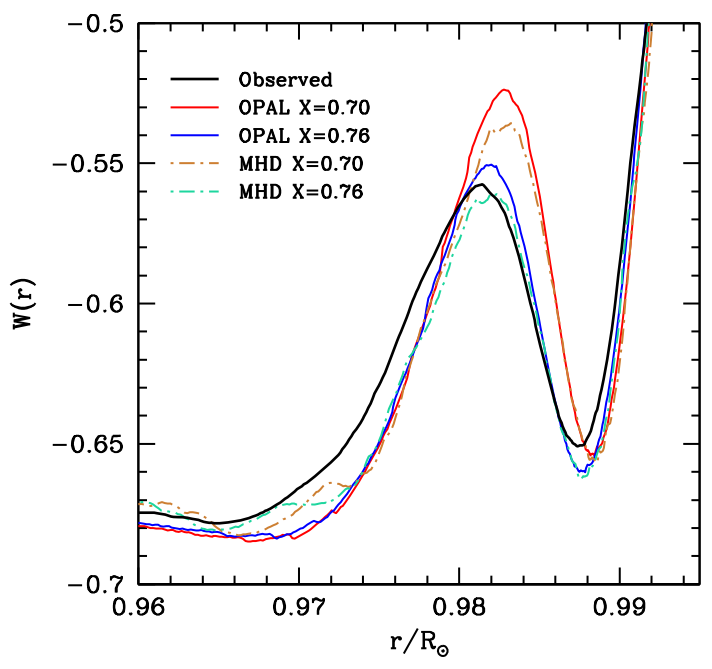

els. Taking into account systematic errors due to the techniques and equation of state effects, they estimated $Y_{s}$ to be $0.249 \pm 0.003$. Basu (1998) used data from the GONG and MDI projects to revise the number to $Y_{s}=0.248$. Basu and Antia (2004) revisited the issue. Instead of looking at $Y_{s}$, they looked at the hydrogen abundance $X_{s}$ and found $X_{s}=0.7389 \pm 0.0034$ using models with $Z / X=0.0171$ in the convection zone.

Most of the effort into determining the solar helium abundance has been directed at determining the present-day helium abundance of the Sun. In terms of stellar evolution though, it is the initial helium abundance that is important. As a result of gravitational settling, we cannot directly estimate what the solar initial helium abundance was. However, using current constraints, it is possible to work backwards to estimate $Y_{0}$ for the Sun. Serenelli and Basu (2010) determined how the initial and present-day helium abundances depend on the parameters used to construct solar models, and then they determined what the limits on the initial helium abundance of the Sun should be given our knowledge of the current structure of the Sun. They found that when only standard solar models are considered, the estimate of the initial helium abundance for the Sun is $Y_{0}=0.278 \pm 0.006$, independent of the solar model or its metallicity. If non-standard models with turbulent mixing below the convection-zone base are used, then $Y_{0}=0.273 \pm 0.006$.

\subsection{Testing input physics}

Helioseismic inversion results can be used to test input physics. In Fig. 5 we showed the frequency-differences between the Sun and two solar models, one that included the diffusion and settling of helium and heavy elements, and one that did not. We had not been able to conclude which of the two models is the better one. The situation changes immediately if we invert the frequency difference to determine the relative sound-speed and density differences between the Sun and the two models. The results of such an inversion are shown in Fig. 20. 

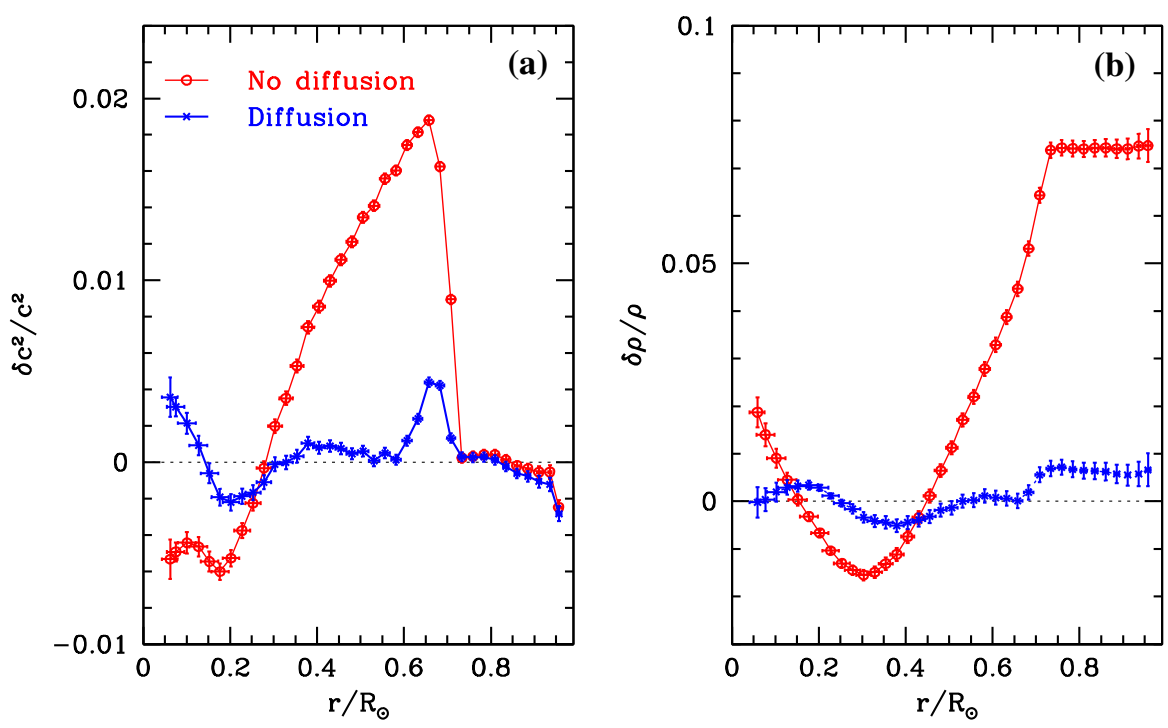

Fig. 20 The relative differences in the squared sound speed (a) and density (b) between the Sun and two solar models obtained by inverting the frequency differences shown in Fig. 5. Note the model without diffusion has much larger differences with respect to the Sun than the model with diffusion

As can be seen from Fig. 20, the model without diffusion is much more discrepant than the one with diffusion. The primary reason for the large discrepancy of the non-diffusion model is that it has a much shallower convection zone than the Sun. Additionally, models without diffusion also have a much higher value of $Y_{S}$ than the Sun. As a result, modern standard solar models include the diffusion and gravitation settling of helium and heavy elements.

In Fig. 6, we showed the frequency differences between models constructed with different equations of state and the Sun and were overwhelmed by the surface term. In Fig. 21, we show the sound-speed difference between the Sun and the different solar models. It is pretty clear that EFF is discrepant, and it appears that OPAL works the best.

Solar models are constructed solving a set of coupled equations and the equation of state affects the structure in multiple ways. As a result, just looking at the soundspeed difference between models constructed with a given equation of state and the Sun does not tell us the full story. A better way is to look at the adiabatic index $\Gamma_{1}$ in the convection zone where we expect adiabatic stratification that depends only on the equations of state. Basu and Christensen-Dalsgaard (1997) had shown that it possible to isolate the effect of the equation of state by inverting Eq. (155). The results of such an inversion are shown in Fig. 22. Note that it is clear that EFF is discrepant. The OPAL equation of state seems to do better in the deeper layers than the MHD equation of state, however, closer to the surface MHD appears to be better. Basu et al. (1999) examined why this is so and concluded that it was a result of how the internal partition function of hydrogen was treated in the two equations of state.

Elliott and Kosovichev (1998) examined the equation of state for conditions present in the solar core. They determined the difference in $\Gamma_{1}$ between models constructed 
Fig. 21 The relative squared sound-speed difference between the Sun and standard solar models constructed with different equations of state. The results were obtained by inverting the frequency differences shown in Fig. 6

Fig. 22 The intrinsic $\Gamma_{1}$ differences between four different equations of state and the Sun. These were obtained by inverting Eq. (155)
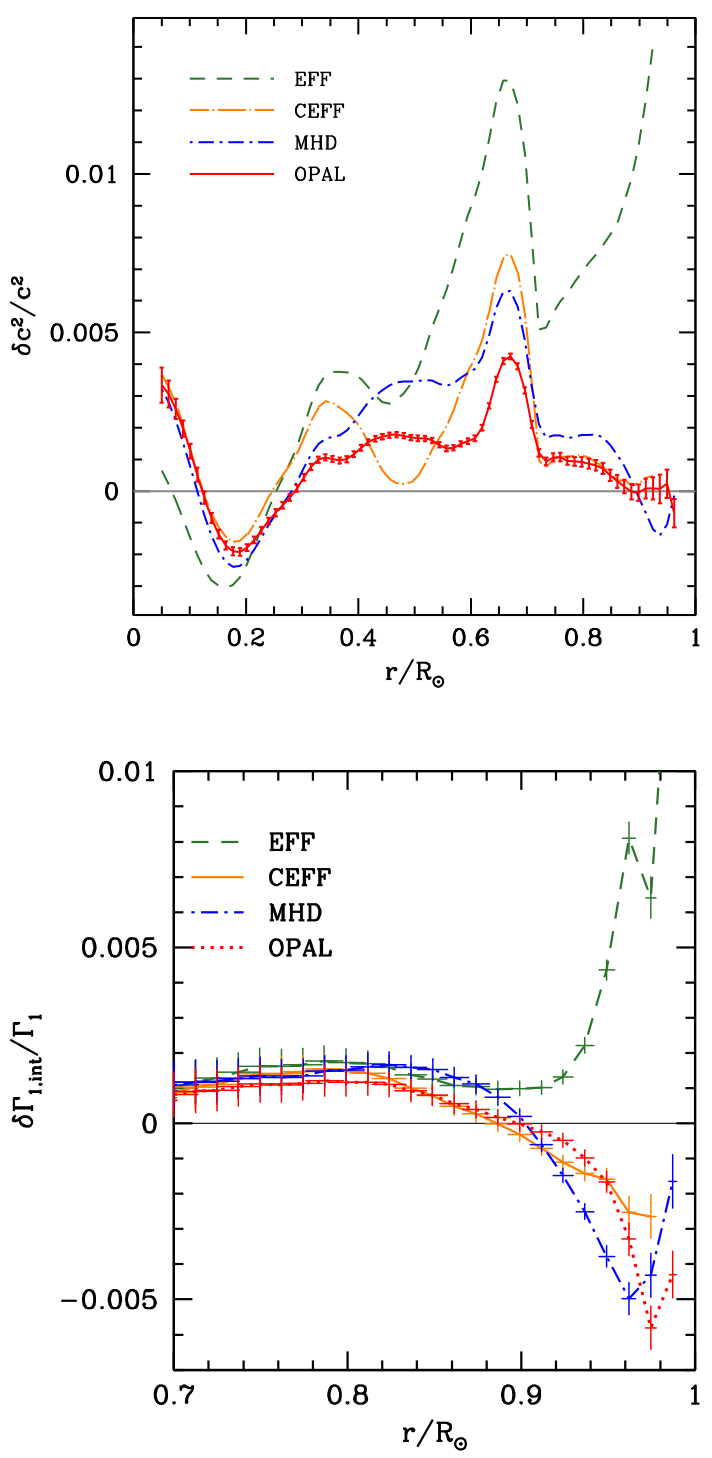

with the MHD and OPAL equations of state and the Sun and found a discrepancy in the core. The cause of the discrepancy was identified as the use of the non-relativistic approximation to describe partially degenerate electrons instead of the relativistic Fermi-Dirac integrals. The deficiency has since been corrected for both OPAL (Rogers and Nayfonov 2002) and MHD (Gong et al. 2001) equations of state.

Somewhat more indirect means have been used to test opacities and nuclear energy generation rates. The stellar oscillation equations do not depend directly on these inputs. What we look for are the effects of changes in these inputs on solar models and how these models look in comparison with the Sun. The earliest tests of opacities 
were simple. Guzik and Cox (1991) compared frequencies of models constructed with the then available opacity tables (those of Cox and Tabor 1976) to suggest that the opacities were too low. Saio (1992) put opacity results on a more quantitative basis by assuming that the sound-speed differences between the models and the Sun were caused by opacity alone. He found that the discrepancy between his models and the Sun can be reduced if the Los Alamos opacities (Weiss et al. 1990) were increased by 20$50 \%$. The publication of the OPAL opacity tables (Rogers and Iglesias 1992) and the OP tables (Badnell et al. 2005) confirmed the results of Saio (1992). Similar methods have been used to look at differences between OPAL opacities and the opacity of the solar material. Tripathy and Christensen-Dalsgaard (1998) using the assumptions of Saio (1992) calculated kernels linking sound-speed changes to opacity changes. Christensen-Dalsgaard et al. (2009) used this method to calculate the opacity changes needed to make low- $Z$ solar models, more specifically models constructed with the Asplund et al. (2005a) metallicities, agree with the Sun and found that changes of up to $30 \%$ may be required. Basu and Antia (1997) looked at density differences rather than sound-speed differences, and found that the density difference between solar envelope models that have the correct convection-zone depth and helium abundance could be used to look at changes in opacity. They concluded that the OPAL opacities were consistent with seismic constraints. It should be noted though that their results were for models with the then accepted solar metallicity of $Z / X=0.0245$. The authors used the same method to later quantity opacity changes needed for models constructed with $Z / X=0.0171$ and found that an increase of about $20 \%$ would be needed to match the density profiles (Basu and Antia 2004).

There have also been attempts to constrain the rate of the $p$ - $p$ reaction. This is the reaction which controls energy generation inside the Sun. Given that the rate of this reaction has not been measured, many helioseismologists turned to seismic data to put constraints on this reaction (see e.g., Antia and Chitre 1998, 1999, 2002; Degl'Innocenti et al. 1998; Schlattl et al. 1999; Turck-chièze et al. 2001). For instance, the seismic constraint on the cross-section of the $p$ - $p$ reaction obtained by Antia and Chitre (2002) (see also Brun et al. 2002) is $S_{11}=(4.07 \pm 0.07) \times 10^{-25} \mathrm{MeV}$ barns. This should be compared to $(4.00 \pm 0.03) \times 10^{-25} \mathrm{MeV}$ barns (Adelberger et al. $1998)$ and $(4.01 \pm 0.04) \times 10^{-25} \mathrm{MeV}$ barns (Adelberger et al. 2011) that has been recommended by nuclear physicists.

Other "secondary" inversion results are those for the abundance profiles inside the Sun. These inversions are done under the assumption that we know the opacity of stellar material perfectly. Kosovichev (1995) determined $\delta X / X$ between the Sun and the model of Christensen-Dalsgaard et al. (1993) and found that the results indicated that the Sun has a smoother gradient of $Z$ below the convection zone than the model. This was confirmed by others. For example, Shibahashi and Takata (1996) assumed $Z / X=0.0277$ and $Y=0.277$ in the solar envelope and varied the $X$ abundance below to determine the $X$ abundance. Antia and Chitre (1998) did a similar analysis. The results showed that the hydrogen abundance below the convection-zone base is smoother than that found in standard solar models. Models constructed with these "seismic" abundance profiles do not have the sharp difference in sound speed at the base of the convection zone that we see in Fig. 16 for standard solar models. 


\subsection{Seismic models}

The fact that the structure of SSMs do not agree completely with that of the Sun have resulted in the construction of so-called "seismic models." These models are constructed so that their structure agrees with that of the Sun. The primary motivation behind early seismic models was better predictions of the solar neutrino fluxes and estimating other properties of the Sun.

There are two types of seismic models. The more common type are seismic models of the present day Sun. These are obtained by solving the stellar structure equations using the helioseismically determined solar sound-speed and density profiles as constraints. The other type consist of models obtained by the usual evolution from the zero-age main sequence, but in these models one or more physics inputs are tuned so that the structure of the final model agrees with that of the Sun. The second type of seismic model is not particularly common; the models of Turck-Chièze et al. (2001) and Couvidat et al. (2003) fall into this category.

One of the earliest examples of the first category of seismic models is that of Kosovichev and Fedorova (1991). Dziembowski et al. (1994, 1995) soon followed with their own models. Antia (1996) constructed a seismic model using an elaborate iterative technique. Seismic models were also constructed by Takata and Shibahashi (1998), Watanabe and Shibahashi (2001) and Shibahashi et al. (1998). Such models are still being constructed as helioseismic data get better; these include the seismic model of Choubey et al. (2001) which was used to estimate properties related to mixing of different neutrino flavours.

\subsection{The solar abundance issue}

In Fig. 16, we showed the sound-speed and density differences between some solar models and the Sun. All the differences were small and we could assume that we are quite good at constructing solar models. All models shown is Fig. 16 however, were constructed assuming that the Sun has a relatively high heavily element abundance. Model $\mathrm{S}$ was constructed using $Z / X=0.0245$ as per the estimates of Grevesse and Noels (1993). The other two models were constructed with $Z / X=0.023$ as per Grevesse and Sauval (1998).

The solar heavy-element abundance is one of the most important inputs to solar models. Since heavy elements increase opacity, the heavy-element abundance affects the structure of solar models. The solar heavy-element abundance is also used as a standard for calibrating the abundances of other stars, as a result, there have been many attempts to determine what the solar metallicity is, and some of the more recent estimates have generated a lot of debate and discussion in the community. In a series of papers Allende Prieto et al. (2001, 2002), and Asplund et al. (2004, 2005b,c) revised the spectroscopic estimates of the solar photospheric composition downwards. The main feature of their analysis was the use of three-dimensional model atmospheres that included the dynamics of the gas and hence obviated the use of micro- and macroturbulence parameters; they also incorporated some non-LTE effects in their analysis. That resulted in lowering the carbon, nitrogen and oxygen abundances in the Sun by 
$35-45 \%$ of those listed in Grevesse and Sauval (1998). The revision of the oxygen abundance leads to a comparable change in the abundances of neon and argon since these abundances are generally measured through the abundances ratio for $\mathrm{Ne} / \mathrm{O}$ and Ar/O. Additionally, Asplund (2000) also determined a somewhat lower value (by about $10 \%$ ) for the photospheric abundance of silicon compared with the value of Grevesse and Sauval (1998). As a result, all the elements for which abundances are obtained from meteoritic measurements have seen their abundances reduced by a similar amount. These measurements have been summarised by Asplund et al. (2005a). The net result of these changes is that $Z$ / $X$ for the Sun reduces to 0.0165 (or $Z \sim 0.0122$ ), about $28 \%$ lower than the previous value of Grevesse and Sauval (1998) and almost $40 \%$ lower than the old value of Anders and Grevesse (1989). These changes in the solar abundance led to very large changes in the structure of solar models. Models constructed with these abundances do not agree well with the Sun. The models have very shallow convection zones and low convection-zone helium abundances.

The mismatch between the low- $Z$ solar models and the Sun led to numerous attempts at modifying the models, and their inputs. There have also been attempts to determine solar abundances from helioseismology. These attempts and their results have been reviewed thoroughly by Basu and Antia (2008). What we give below is an update of the situation. With further improvement in analysis, abundances were updated by Asplund et al. (2009) to $Z / X=0.018$. While this improved the models somewhat, they were by no means as good as those with higher values of $Z / X$ (see e.g., Serenelli et al. 2009). There were other, independent, attempts to determine solar heavy element abundances using three-dimensional model atmospheres, and this led to $Z / X=0.0209$ (Caffau et al. 2010, 2011). In Fig. 23, we show the relative soundspeed and density differences between the Sun and solar models constructed with different heavy-element abundances. It is clear that the low- $Z$ models do not match. Some of the properties of these models are listed in Table 3. Basu and Antia (2013) used solar envelope models and density inversion results to determine the amount how much opacity increase would be needed to make the models in Fig. 23 agree with the Sun; they found changes of $6 \%$ for the model with Caffau et al. $(2010,2011)$ abundances, $16.5 \%$ for the Asplund et al. (2009) model and 26.5\% for the Asplund et al. (2005a) models. A recent review of the issue can be found in Bergemann and Serenelli (2014).

Since the Basu and Antia (2008) review was published there have been more investigations to see whether low- $Z$ models can be brought in agreement with the Sun without too many changes to the inputs. One such attempt was by Zhang (2014) who showed that including the dynamical effects of convection, in particular the kinetic energy flux, could make low- $Z$ models agree with the Sun. While the scope of that investigation was very limited and not applied to SSMs but only to envelope models, and it is not clear if the kinetic energy profile used is realistic, it is certainly an interesting, physically motivated idea. Another, somewhat unconventional, idea was proposed, and ultimately rejected, by Vincent et al. (2013). They examined the impact of particles with axion-like interactions with photons and whether they affect the spectroscopically determined values of the solar heavy-element abundance. They found that these interactions could in principle resolve the problem by inducing a slight increase in the continuum opacity at line-forming heights in the solar atmosphere. 

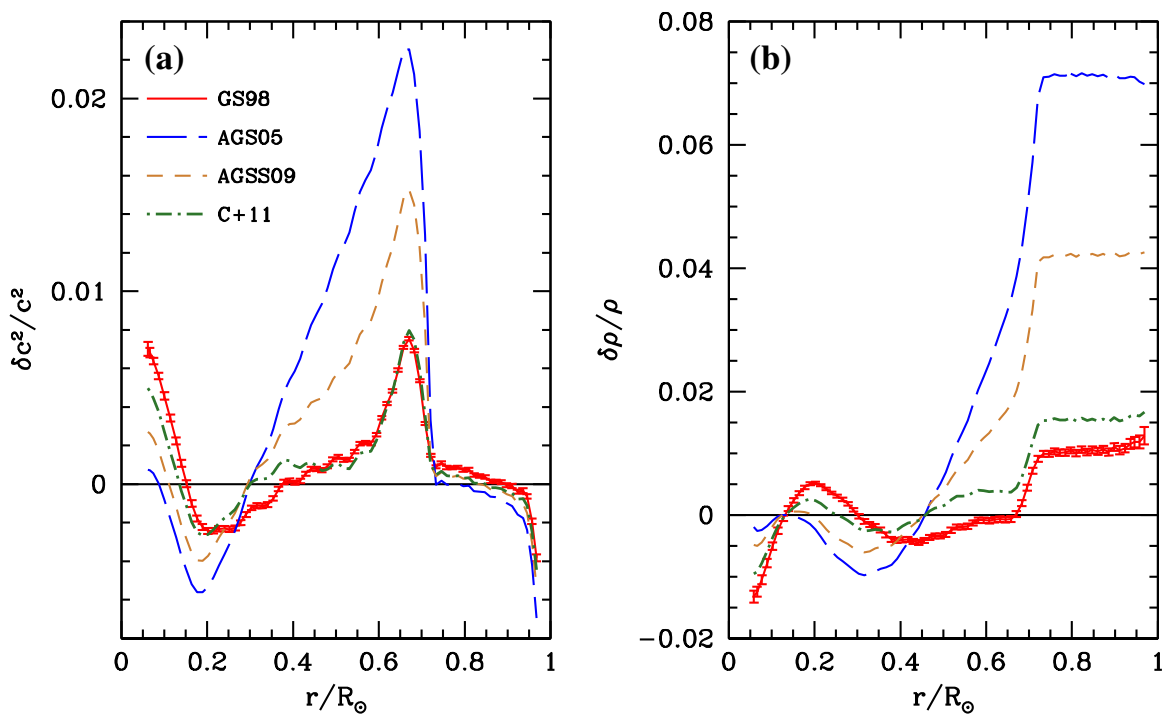

Fig. 23 The relative sound-speed and density differences between the Sun and four standard models constructed with different heavy-element abundances but otherwise identical physics inputs. The model GS98 was constructed with Grevesse and Sauval (1998) abundances, AGS05 with Asplund et al. (2005a) abundances, AGSS09 with Asplund et al. (2009) abundances, and models C+11 was constructed with Caffau et al. $(2010,2011)$ abundances supplemented with abundances from Lodders (2010)

Table 3 Properties of standard solar models shown in Fig. 23

\begin{tabular}{lllll}
\hline Mixture & $Z / X$ & $R_{\mathrm{CZ}}$ & $Y_{\mathrm{CZ}}$ & $Y_{0}$ \\
\hline Helioseismic & - & $0.713 \pm 0.001^{\mathrm{a}}$ & $0.2485 \pm 0.0034^{\mathrm{b}}$ & $0.273 \pm 0.006^{\mathrm{c}}$ \\
GS98 & 0.023 & 0.7139 & 0.2456 & 0.2755 \\
AGS05 & 0.0165 & 0.7259 & 0.2286 & 0.2586 \\
AGSS09 & 0.018 & 0.7205 & 0.2352 & 0.2650 \\
C+11 & 0.0209 & 0.7150 & 0.2415 & 0.2711 \\
\hline
\end{tabular}

${ }^{\mathrm{a}}$ Basu and Antia (1997); ${ }^{\mathrm{b}}$ Basu and Antia (2004); ${ }^{\mathrm{c}}$ Serenelli and Basu (2010)

This in turn would reduce the computed equivalent widths of solar absorption lines for any given elemental abundance, which would result in an underestimation of the abundances. However, the authors found that the coupling necessary to obtain the required changes are ruled out by current experiments.

There have been more efforts to determine the solar abundance independently of spectroscopy. Vorontsov et al. (2013), using solar envelope models and the adiabatic index $\Gamma_{1}$ claim that helioseismic data are consistent with low (and even very low) metallicities in the range $Z=0.008-0.013$. This contradicts the results of Antia and Basu (2006). These low- $Z$ results also contradict what was found by Villante et al. (2014) who did a joint statistical analysis of helioseismic and solar neutrino data to find that the solar abundances of oxygen and iron are consistent at the $\sim 1 \sigma$ level with 

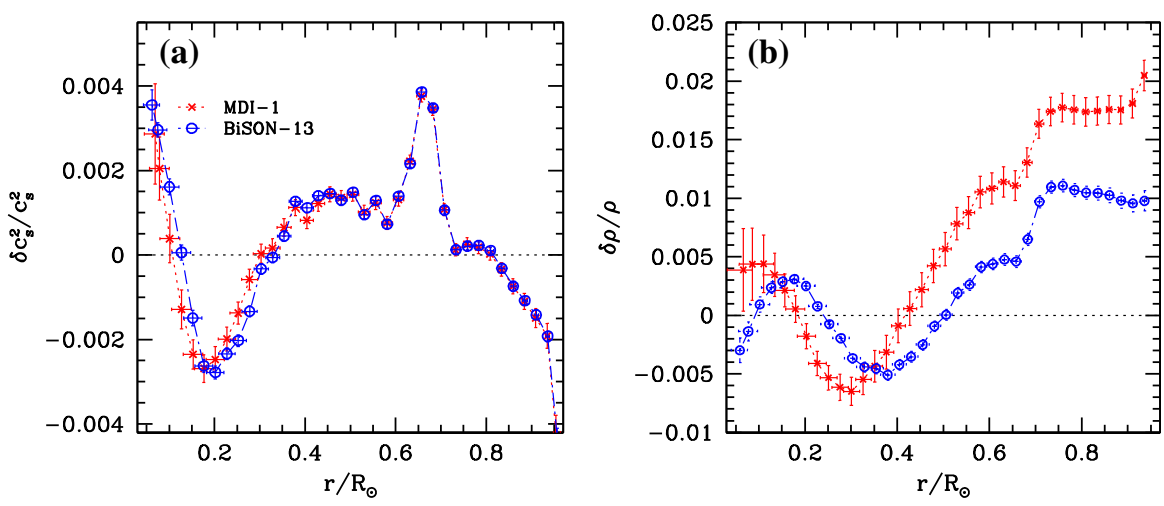

Fig. 24 The relative sound-speed and density differences between the Sun and Model S obtained by inverting two different data sets that only differ in their $\ell=0,1,2$, and 3 modes. The results are from Basu et al. (2009) and the two mode sets, labelled MDI-1 and BiSON-13 are described in that paper. Note that while there is very little change in the sound-speed differences, the density differences change significantly

(high) values of Grevesse and Sauval (1998). Solar $Z$ values as low as 0.008 are also inconsistent with current models of Galactic chemical evolution.

Thus the solar abundance issue is still alive and well! Reliable high-degree mode frequencies will help in resolving the near-surface layers of the stars where the ionisation zones of different elements leave their imprint. Until then we have to look at multiple diagnostics and determine whether we can reach a consensus on this issue.

\subsection{How well do we really know the structure of the Sun?}

Inversions of solar p mode frequencies available today allow us to determine the structure of the Sun between about 0.05 to $0.96 R_{\odot}$ reliably. There are a few (e.g., Di Mauro et al. 2002) that go closer to the surface, but these results are geared towards studying near-surface features. The interior limit is set by the available low degree modes, while the near-surface limit is set by the lack of reliable high-degree modes. For the intervening layers, different data sets give consistent sound-speed inversion results. As a result, we believe that we know the solar sound-speed profile quite well. This is not the case for density-improvements in the mode sets, particularly in the low-degree range, change the density inversion results in a statistically significant manner. This can be seen in Fig. 24. The reason for this is the conservation of mass condition. Since the models are constructed to have the same mass as the Sun, the integrated density difference between the Sun and the models should be zero. As a result when mode sets improve, the density results change at all radii. Improvements of low-degree modes are particularly important as they give better results for the core. Since density is highest in the core, a small change in the inferred densities in that regions results in a large change elsewhere.

Better results for the solar core can be obtained with g modes (see e.g., Kosovichev 1988a). These modes have high amplitudes in the core and hence, sample the core well. However, as mentioned in Sect. 3, g-mode amplitudes decay in convection zones 
and the Sun has a fairly deep one, making the task of detecting g modes very difficult. There is a long history of trying to detect solar g modes and a short report of the rather frustrating quest can be found in Appourchaux and Pallé (2013). For a more technical review see Appourchaux et al. (2010).

García et al. (2007) claim that they detect periodic structures that was consistent with the expected splitting for dipole g modes in periodograms constructed with GOLF data. That work did not list individual frequencies or frequency-splittings. García et al. (2008) showed that there was statistically significant power in the same frequency range in the VIRGO data. There is no consensus yet as to whether or not individual g mode peaks can be detected with the data we have today, and for that matter, whether or not their frequencies can be measured. García (2010) claims tentatively that the individual modes can be seen. This is the only group that has claimed to have measured frequencies of individual $\mathrm{g}$ modes and not merely their signature in modern, highprecision data. If their results are confirmed independently, and the frequencies are measured to a good precision, we should be able to get a better handle on the structure of the solar core and the density of all layers of the Sun.

The other part of the Sun which we have not probed very well yet is close to the solar surface, i.e., the near-surface layers. The reason is the dearth of reliable high-degree modes. Most helioseismic data sets have mode frequencies of up to $\ell=200$ (GONG) or $\ell=250$ (MDI and HMI). These modes do not probe the outer layers very well. Having high-degree modes assists the inversions by better defining the surface term by resolving differences close to the surface (Rabello-Soares et al. 2000). The reason for the lack of modes is technical: above about $\ell=200$, individual modes have large line-widths due to their short lifetimes, and they merge into ridges when combined with the observational spatial leakage. The spatial leakage is the spherical-harmonic transform equivalent of the side lobes seen in Fourier transforms. The leakage occurs because a spherical harmonic decomposition is not orthogonal when using data over the visible solar disc, which is less than half a hemisphere. This results in modes of similar degrees and azimuthal orders leaking into the estimate of a specific degree and azimuthal order. Other reasons of leakage include line-of-sight projection effects, distortion of modes by differential rotation, etc. For low- $\ell$ models the leakages are separated in frequency domain, but the large widths of high-degree modes means that the leakages and the central peak form a ridge. Asymmetries in the leakage could mean that the highest point of the ridge may not be the eigenfrequency of the Sun. Thus determining frequencies of high degree modes is challenging (see, e.g., Rhodes et al. 2003; Reiter et al. 2003; Korzennik et al. 2013b). At lot of effort is being put into this, however, progress has been slow.

\section{Departures from spherical symmetry}

The Sun is spherical in shape- the measured difference between the polar and equatorial radii is only about 1 part in $10^{5}$ as per the analysis by Kuhn et al. (1998) of MDI data. Updated results obtained with data from the PICARD satellite puts the number slightly lower, a difference of $6.1 \mathrm{~km}$, i.e., about 9 parts in $10^{6}$ (Irbah et al. 2014) or perhaps even lower $(5.7 \pm 0.2 \mathrm{~km}$, Meftah et al. 2015). However, although spherical, 
the Sun is not spherically symmetric. The main deviation from spherical symmetry in the solar interior is caused by rotation; on the solar surface, deviations from spherical symmetry are also seen in magnetic fields.

Rotation, magnetic fields and anything else that breaks spherical symmetry causes oscillation frequencies to split and lifts the degeneracy between frequencies of modes that have the same degree $\ell$ and radial order $n$ but that have different values of the azimuthal order $m$ (Ledoux 1949; Ledoux and Walraven 1958; Cowling and Newing 1949). These splittings can be used to determine solar rotation. The frequency splittings $v_{n, \ell, m}-v_{n, \ell, 0}$ can be separated into two components, one that is odd in $m$ and one that is even in $m$. The odd component arises from rotation, and in particular from advection and Coriolis force. The component even in $m$ is caused by centrifugal forces, largescale magnetic fields or any non-spherically symmetric distortion of the star.

As mentioned in the introduction, it is customary to write the frequencies and their splittings as a polynomial expansion in $m$, i.e.,

$$
\frac{v_{n \ell m}}{2 \pi}=v_{n \ell m}=v_{n \ell}+\sum_{j=1}^{j_{\max }} a_{j}(n \ell) \mathcal{P}_{j}^{n \ell}(m),
$$

where $\mathcal{P}_{j}^{n \ell}(m)$ are related to Clebsch-Gordon coefficients. In this representation, the odd-order $a$-coefficients are sensitive to rotation. This is a result of the structure of the kernels that relate the frequency splittings to rotation (for a description of the kernels see Christensen-Dalsgaard 2003). The $a_{1}$ coefficient encodes the rotation rate averaged over all latitudes; $a_{3}$ and higher order coefficients describe differential rotation. The even-order coefficients are sensitive to the second-order effects of rotation (i.e., the centrifugal forces) as well as magnetic fields and other deviations from spherical symmetry. The actual values of the $a$ coefficients at a given epoch depends on the polynomials $\mathcal{P}$ used in the expression given in Eq. (157). The most common practice today is to use either the Clebsch-Gordon coefficients or the Ritzwoller and Lavely (1991) formulation, or polynomials proportional to those. Connections between some of the more common definitions of the coefficients may be found in Pijpers (1997).

In the case of the Sun, the first odd-order coefficient $a_{1}$, is much larger than the first even-order coefficient (see Fig. 25) indicating that rotation is the primary agent for breaking spherical symmetry in the Sun.

\subsection{Solar rotation}

For a detailed review of solar rotation, readers are referred to the Living Reviews in Solar Physics article by Howe (2009); we describe the basic features here.

The motions of sunspots on the solar disk had revealed that the surface of the Sun rotated differentially with the solar equator rotating once every 25 days and the higher latitudes taking a longer time to rotate. This had been confirmed from Doppler shifts of photospheric lines (Snodgrass 1983, 1984; Ulrich et al. 1988, etc.), as well as measurements with other tracers (e.g., Duvall 1980; Snodgrass and Ulrich 1990; Komm et al. 1993). It had been predicted that the rotation would be constant on cylindrical 
Fig. 25 The dominant odd-order splitting component $\left(a_{1}\right.$; blue triangles) and the dominant even-order component ( $a_{2} ;$ red crosses) for the Sun. Data are from a MDI frequency set constructed with a 1-year time series from observations that began on May 1, 1996. Note that the $a_{1}$ component is much larger than the $a_{2}$ component, indicating that rotation is the main agent for breaking spherical symmetry in the Sun

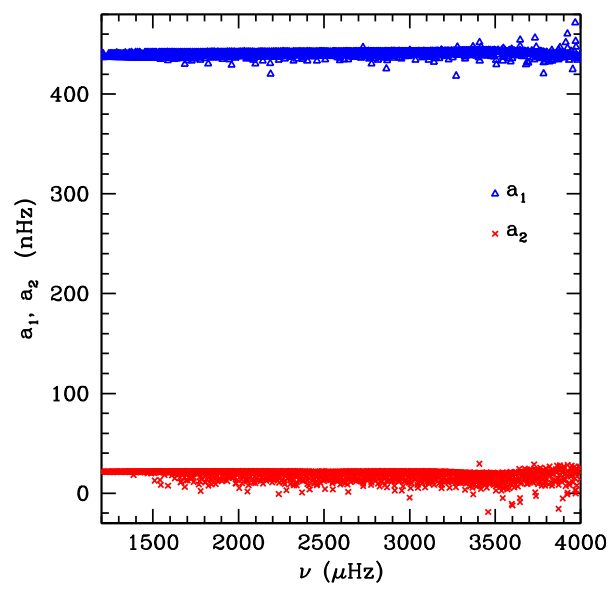

Fig. 26 The mean solar rotation profile obtained with data collected by the GONG project between mid-1995 to mid-2004. The rotation rate in the deep interior has large uncertainties and hence, has not been shown. The inversion results are from Howe et al. (2005). (Image courtesy of Rachel Howe)

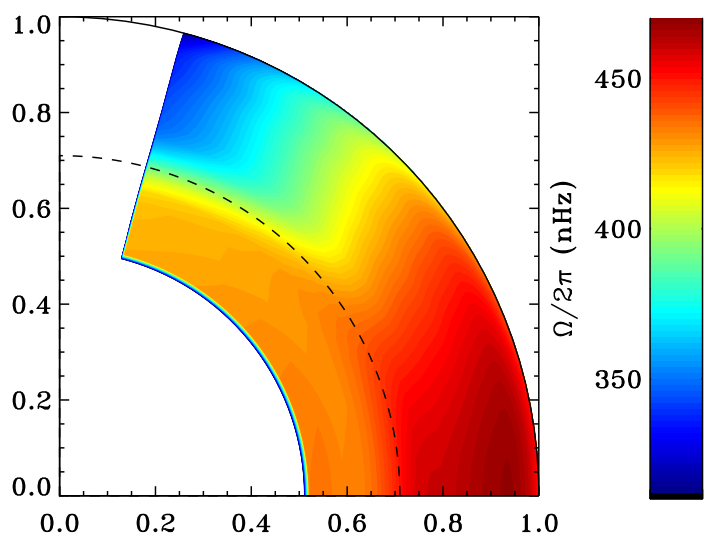

surfaces throughout the convection zone, matching onto the observed surface rotation at the top of the convection zone, i.e., the Sun iso-rotation contours of the Sun will be cylinders. Helioseismology revealed a very different, and more complicated, picture (see e.g., Thompson et al. 1996; Schou et al. 1998a, and references therein). The mean rotation profile of the solar interior is shown in Fig. 26. A few features stand out immediately. The Sun rotates like a solid body below the base of the convection zone, though the rotation in the innermost parts of the Sun is not really known because it is so poorly constrained by the observations of $\mathrm{p}$ modes. Within the convection zone rotation changes with both latitude and radius. For most of the convection zone, rotation is nearly constant on cones, i.e., at fixed latitude the rotation is almost independent of depth, however, there is some hint of rotation on cylinders in the near-equatorial regions of the convective envelope (Gilman and Howe 2003). There are two distinct shear layers, one in the immediate sub-surface layers, and a more pronounced one at the base of the convection zone. The shear layer at the base of the convection zone is now called the "tachocline." For a review of different methods of determining solar rotation, see Beck (2000). 
The data that yield the solar rotation rate have also been used to determine the total angular momentum, $H$, of the Sun, its total rotational kinetic energy, $T$, as well as the gravitational quadrupole moment, $J_{2}$. Pijpers (1998) showed that the error-weighted mean of these quantities obtained with GONG and MDI data are:

$$
\begin{aligned}
H & =(190.0 \pm 1.5) \times 10^{39} \mathrm{~kg} \mathrm{~m}^{2} \mathrm{~s}^{-1}, \\
T & =(253.4 \pm 7.2) \times 10^{33} \mathrm{~kg} \mathrm{~m}^{2} \mathrm{~s}^{-2}, \\
J_{2} & =(2.18 \pm 0.06) \times 10^{-7}
\end{aligned}
$$

These were redetermined by Antia et al. (2000b) and they obtained consistent, though slightly different results.

\subsection{Other deviations from spherical symmetry}

While solar rotation has been studied in great detail, other asphericities in the Sun have not been the subject of many detailed studies. The reason for this is twofold, the first being that the signature of such asphericities in the Sun is small, as is clearly seen in Fig. 25. The second reason is perhaps the more fundamental one: the even- $a$ coefficients have the disadvantage that the signal in the coefficients could be a result of sound-speed asphericities or magnetic fields or both. Zweibel and Gough (1995) showed that even-order frequency splittings cannot be unambiguously attributed to the effect of a magnetic field, and the effect of a magnetic field on frequency splittings can be reproduced by a perturbation of the sound speed. Zweibel and Gough (1995) argued that the ambiguity in the signature of global models arises because the range of latitudes sampled by the modes depends on their azimuthal order $m$. This allows both magnetic and acoustic perturbations to produce the same frequency shifts. However, the spatial configuration of a magnetic field that produces a given splitting is not the same as the spatial configuration of an acoustic perturbation that produces the same splitting. As a result, studies of asphericity using frequency splitting proceed by assuming that the even-order splittings or $a$ coefficients are caused by magnetic fields alone or by aspherical perturbations to structure alone. Most modern investigations however, do correct for the second-order effects of rotation. It should be noted that asphericities in the shape of the Sun can also produce even-order splittings (Gough and Taylor 1984), however, the measured asphericity of the Sun's limb is smaller than what is needed to explain the observed $a$ coefficients. For a review on how magnetic fields can affect mode frequencies and splittings see Thompson (2006).

\subsubsection{Magnetic fields}

One of the first estimates of solar interior magnetic fields obtained with frequency splittings was that of Dziembowski and Goode (1989). They developed a formalism relating the frequencies to the magnetic field and used the data from Libbrecht (1989) to find that there should be an axisymmetric quadrupole toroidal magnetic field of $2 \pm 1 \mathrm{MG}$ centred near the base of the convection zone. They confirmed the results using data from Libbrecht and Woodard (1990) (Dziembowski and Goode 1991). 
Goode and Thompson (1992) tested the hypothesis that the radiative interior of the Sun could be hiding a large-scale magnetic field, which might not be axisymmetric about the observed rotation axis and found that the strength of an axisymmetric relic field must be less than $30 \mathrm{MG}$. Such a field will cause an oblateness of 5-10 $\times 10^{-6}$. Goode and Dziembowski (1993) revisited their older work and using updated data for the splittings found that the significance of the mega-Gauss field at the convectionzone base was lower than what they had suggested in their earlier papers. Their result was revised further by Basu (1997) who used different data (those obtained by the GONG project), and a somewhat different method, to find an upper limit 0.3 MG on the toroidal magnetic field concentrated below the convection-zone base.

Gough and Thompson (1990) presented a perturbation method to calculate the effects of magnetic fields and rotation on the frequency splittings. Modified versions of that formulation have been used extensively to estimate the magnetic fields in the interior of the Sun. Using the precise $a$ coefficients obtained by the GONG and MDI projects, Antia et al. (2000b) used the Gough and Thompson (1990) formulation to do a forward analysis and showed that the even-order $a$ coefficients, when corrected for the second-order effects of rotation, can be explained by a $20 \mathrm{KG}$ field located at a depth of $30,000 \mathrm{~km}$ below the solar surface. They also found an upper limit of $0.3 \mathrm{MG}$ for a toroidal field at the base of the convection zone. Antia (2002) used the data to put an upper limit of $10^{-5}$ on the ratio of magnetic pressure to gas pressure in the solar core. Baldner et al. (2009) did a more detailed analysis; they used multiple data sets and found that the data are best fit by a combination of a poloidal field and a doublepeaked near-surface toroidal field. The toroidal fields are centred at $r_{0}=0.999 R_{\odot}$ and $r_{0}=0.996 R_{\odot}$ and have peaks strengths of $380 \pm 30 \mathrm{G}$ and $1.4 \pm 0.2 \mathrm{KG}$ respectively. The poloidal field is best described by a dipole field with a peak strength of $124 \pm 17 \mathrm{G}$. It should be noted that the idea of a shallow field goes back a long way-in an IAU Symposium in 1986, Gough and Thompson showed how the then-available data have hints of a shallow perturbation (see Gough and Thompson 1998).

\subsubsection{Acoustic asphericity}

As mentioned earlier, even-order splitting coefficients can also be caused by aspherical acoustic perturbations, and hence, there have been a number of investigations aimed at estimating the acoustic asphericity, if any, of the Sun. Early works (Kuhn 1988a, 1989) related the frequency splittings to temperature perturbations, but the data were not good enough to make firm conclusions. Data from GONG and MDI changed the situation somewhat, however, there are still very few studies of asphericity of solar internal structure.

Basu and Antia (2001b) used a combination of the central frequencies and the evenorder $a$ coefficients to make frequency combinations pertaining to different latitudes and used those to determine the position of the base of the convection zone as a function of latitude. They did not find any statistically significant difference in the position with latitude. Antia et al. (2001a) did a detailed analysis of all the sets of splittings available to determine the latitudinally dependent sound-speed profile of the Sun by doing a two-dimensional inversion of the even-order $a$ coefficients. They found that it was possible to determine structural asphericities within the convection 
zone, though the errors became progressively larger the deeper they went. They found sound-speed asphericities of the order of $10^{-4}$. The most notable feature is a peak in the asphericity around $0.92 R_{\odot}$. The other feature is that sound-speed perturbation at the equator is negative, but it is positive at mid-latitudes, a results which can be interpreted as the equator being cooler than the mid-latitude region. The amount of the temperature asphericity is similar to that found by Dziembowski et al. (2000) who inverted the different orders of the coefficients, i.e., $a_{2}, a_{4}$ etc., separately.

Asphericity in the near-surface layers is expected to be larger because the low densities there make the layers susceptible to perturbations. However, the absence of reliable high-degree mode-frequencies makes it difficult to study these regions.

\section{Solar-cycle related effects}

One of the more interesting features of solar oscillations is that the oscillation frequencies change with solar activity. This was noted even in early data (Woodard and Noyes 1985; Woodard 1987; Libbrecht 1989; Pallé et al. 1989; Elsworth et al. 1990b; Libbrecht and Woodard 1990; Anguera Gubau et al. 1992). Modern data have confirmed the early results. The solar-cycle related changes affect the central frequencies and frequency splittings of low degree modes (Salabert et al. 2004; Toutain and Kosovichev 2005; Chaplin et al. 2007) as well as intermediate degree modes (Bachmann and Brown 1993). Although not too many high-degree data sets are available, it is clear that there are changes in the frequencies of high-degree modes too (Rabello-Soares et al. 2006, 2008; Rhodes et al. 2011). The frequency changes appear to be functions of frequency alone when mode inertia is taken into account, and is reminiscent of a "surface term" (see Fig. 27). Frequency splittings also show solar-cycle related shifts.

Woodard et al. (1991) showed that changes in solar frequencies were strongly correlated with the changes in the magnetic field strength. This was subsequently confirmed by Bachmann and Brown (1993), Elsworth et al. (1994) and Régulo et al. (1994). The variation of the frequencies with the changing magnetic activity is now

Fig. 27 The scaled frequency differences between two sets of solar data, one obtained at solar maximum and the other at solar minimum. The frequencies were obtained by MDI, each from a 72-day long time-series. The one corresponding to solar maximum was from observations that began in June 2001 (MDI set 3088), the one for the minimum was from observations that began in May 1996 (MDI set 1216). (Data courtesy of Tim Larson)

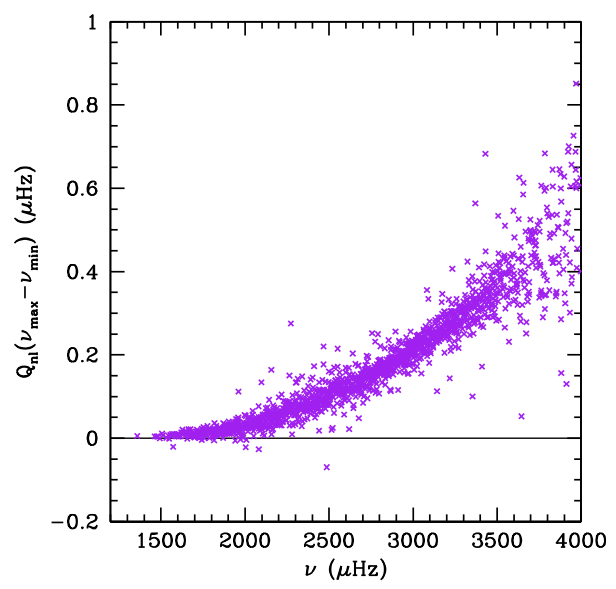


well established and its nature being looked into in detail. Although solar oscillation frequencies increase with increase in solar activity, the rise and fall does not always follow the same path. Jiménez-Reyes et al. (1998) analysed low-degree cycle 22 data to find a "hysteresis" effect. This was also seen by Tripathy et al. (2001) in intermediatedegree data. Like Jiménez-Reyes et al. (1998), they found that some of the solar activity indices also show this effect. However, the interesting feature shown by Tripathy et al. (2001) is that while magnetic indices (like KPMI, the Kitt Peak Magnetic Index and MPSI, the Magnetic Plage Strength Index) show the same type of hysteresis as solar frequencies, some of the radiative indices of solar activity do not. For the 10.7-cm flux as well as the equivalent width of the He $10830 \AA$ line, the ascending and descending branches cross each other.

The relationship between solar frequency shifts and solar activity indices is however, not always simple. Most investigations assume a linear relationship between the different indices and frequency shifts, there are however, significant deviations and anomalies. Tripathy et al. (2007) looked at the correlation between frequency shifts and magnetic indices over different time periods and showed that the correlation varies from their to year. They also demonstrated that there were significant differences between long-term and short-term variations and that the correlation complex. Deviations from a simple trend were also found by Howe et al. (2006a) who studied at the frequency shifts for modes of $\ell=0-2$ observed by BiSON, GONG and MDI, and found that even after a linear solar-activity dependence is removed, there are significant variations can are seen in the different data sets. The authors conclude that the variations are likely to be related to the stochastic excitation of the modes. Chaplin et al. (2007) looked at three solar cycles worth of data from BiSON and compared the behaviour of mean frequency shifts of low- $\ell$ modes with several proxies of global solar activity to determine, among other things, which activity proxy follows the frequency shifts most closely. They assumed a linear correlation and concluded that the $\mathrm{He}$ I equivalent width and the $\mathrm{Mg}$ II core-to-wing data had the best correlation, though the 10.7-cm flux also fared well. This led to an in-depth examination of the shifts of intermediate degree modes by Jain et al. (2009). Using data from GONG and MDI they examined the correlation between the frequency shifts and nine different activity indices for cycle 23. Their updated results are summarised in Fig. 28. They also divided their data into a rising phase (1996 September 22 to 1999 June 26), a high activity phase (1999 June 27 to 2003 January 12) and a declining phase (2003 January 13 to 2007 July 26) and found that the correlation between frequency shifts and solar activity changes significantly from phase to phase except in the case of the 10.7-cm flux. In most cases, the rising and the declining phases were better correlated than the high-activity phase. The different degrees of correlation of the different activity indices with the frequency shifts should, in principle, inform us about changes in the Sun, however, interpreting the correlations has proved to be difficult.

The Sun also exhibits variability of time scales much shorter than the usual 11-year solar cycle. Over the last few decades, it has been suggested that the Sun shows significant variability on a shorter time scale of about 2 years. Benevolenskaya (1995) and Benevolenskaya (1998b) showed this using low-resolution magnetic field observations taken at the Wilcox Solar Observatory. Mursula et al. (2003) reported periods between 1 and 2 years in various heliospheric parameters such as solar wind speed, 

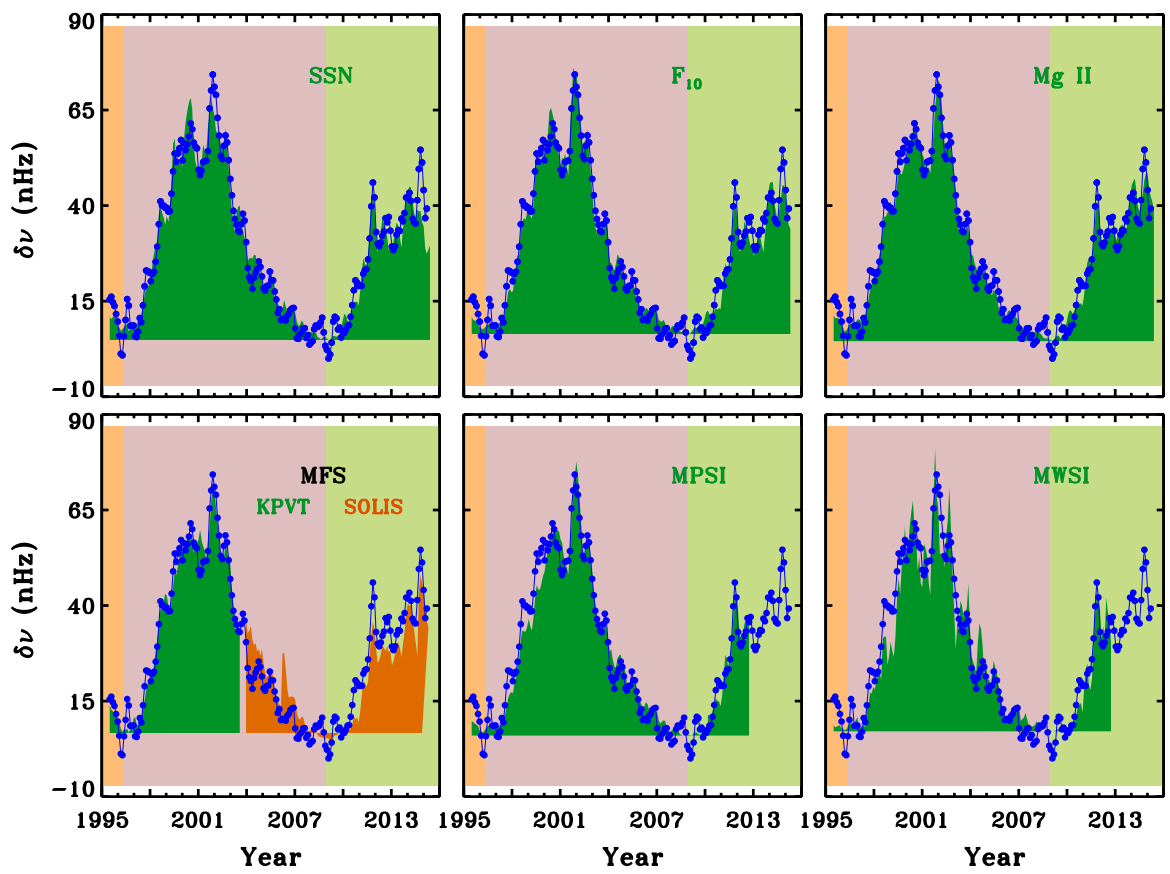

Fig. 28 The time-evolution of the GONG frequency shifts (points) plotted with different activity proxies (filled regions) scaled to fit on the same axis. The frequency shifts were calculated with respect to the temporal mean of the frequencies. The orange, pink and light-green backgrounds denote solar cycles 22 , 23 and 24 respectively. Figure courtesy of Kiran Jain

interplanetary magnetic fields, geomagnetic activity etc. Valdés-Galicia and Velasco (2008) showed that such 1-2 year variabilities are also seen in coronal holes and radio emissions. Readers are referred to the Living Reviews in Solar Physics article of Hathaway (2015) for details of the quasi-biennial period in different observations. Such short-term variations are also seen in solar frequencies. Fletcher et al. (2010) showed that solar frequencies are modulated with a 2 year period. This was seen in data from both BiSON and GOLF (see Fig. 29). Note that the amplitude of the modulation is almost independent of the frequency of the mode. This is quite unlike the main solar-cycle related modulation where high-frequency modes show a larger change of frequencies than low-frequency modes. However, the amplitude of the modulation shows a time-variation: the amplitude is larger at solar maximum than at solar minimum. Fletcher et al. (2010) and Broomhall et al. (2011) speculated that the 2-year signal is that from a second dynamo whose amplitude is modulated by the stronger 11 year cycle. The almost frequency-independent nature of the 2-year signal points to a second dynamo whose seismic effects originate at layers deeper than those of the primary dynamo. The presence of two different types of dynamos operating at different depths has already been proposed to explain the quasi-biennial behaviour observed in other proxies of solar activity (Benevolenskaya 1998a, b). However, not everybody agrees at the second signal is that of a second dynamo; Simoniello et al. (2013) claim 

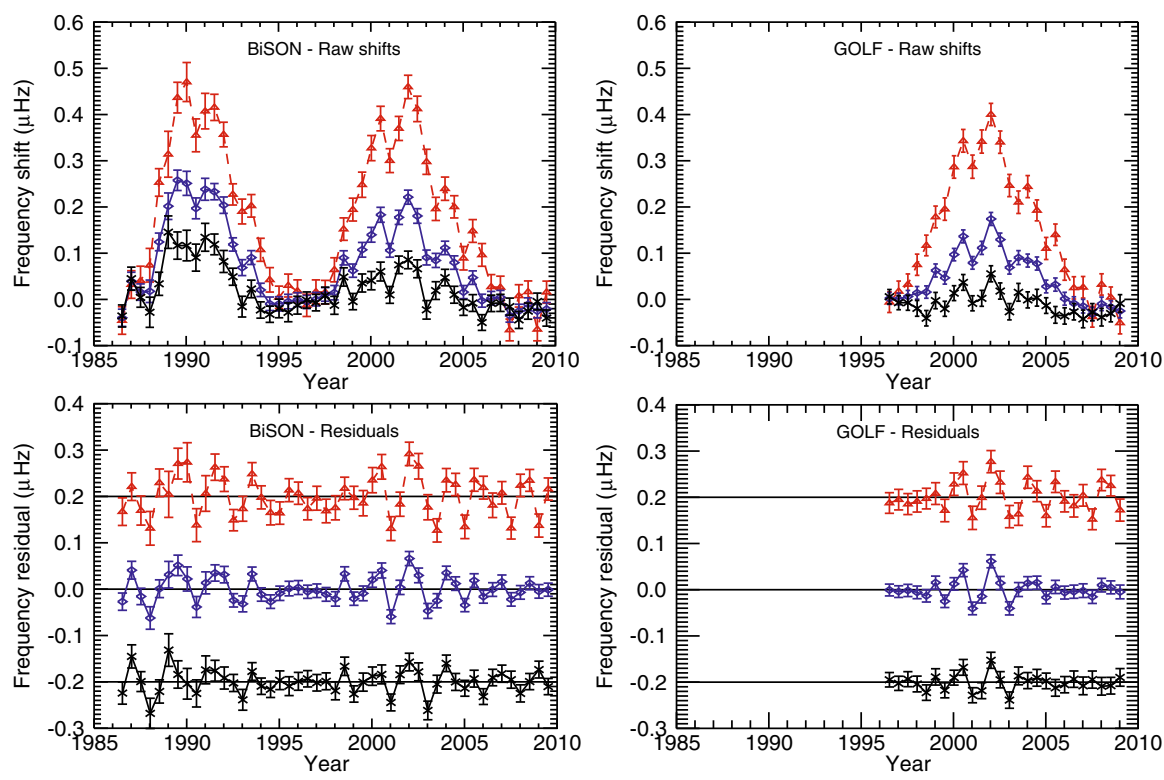

Fig. 29 The upper panels show the shifts in frequencies of low-degree modes from BiSON (left) and GOLF (right) as a function of time. The blue solid line is for all modes with frequencies between 1.88 and $3.71 \mathrm{mHz}$. The black and red lines are subsets: black for 1.88 to $2.77 \mathrm{mHz}$ and red from 2.78 to $3.71 \mathrm{mHz}$. The lower panels show the residuals when the dominant solar-cycle trend is removed. The black and red curves have been displaced by 0.2 and $-0.2 \mathrm{mHz}$ respectively for the sake of clarity. (Image reproduced with permission from Fletcher et al. 2010, copyright by AAS)

that the signal could result from the beating between a dipole and quadrupole magnetic configuration of the solar dynamo.

\subsection{Were there solar cycle-dependent structural changes in the Sun?}

The apparent lack of a degree dependence in the frequency shifts have led to the conclusion that solar cycle related changes in the Sun are confined to a thin layer close to the surface or even above the surface (Libbrecht and Woodard 1990; Goldreich et al. 1991; Evans and Roberts 1992; Nishizawa and Shibahashi 1995, etc.). This picture was confirmed by subsequent studies (e.g., Howe et al. 1999; Basu and Antia 2000; Dziembowski and Goode 2005). Theoretical work too suggested shallow changes (e.g., Goldreich et al. 1991; Balmforth et al. 1996; Li et al. 2003). There have been attempts to determine the location of the changes, and with data available over more than a solar cycle, and it has indeed been possible to detect some of changes.

Goldreich et al. (1991) and Gough (2002) had suggested that there could be changes around the second helium ionisation zone. This does seem to be the case. Basu and Mandel (2004) used the fourth-differences of the frequencies obtained by GONG and MDI, i.e.,

$$
\delta^{4} v_{n, \ell}=v_{n+2, \ell}-4 v_{n+1, \ell}+6 v_{n, \ell}-4 v_{n-1, \ell}+v_{n-2, \ell} .
$$




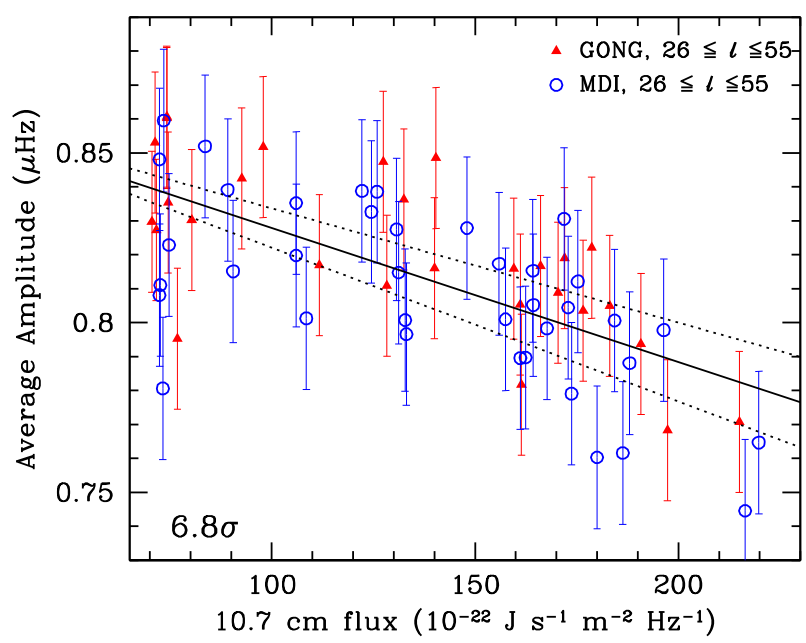

Fig. 30 The average amplitude of the oscillatory signal in the 4th differences of different MDI and GONG data sets plotted as a function of the $10.7-\mathrm{cm}$ flux. The average was calculated in the frequency range $2-3.5 \mathrm{mHz}$. The results were obtained for the degree range $26 \leq \ell \leq 55$. The black continuous line is a least-squares fit to all the data points. The dotted lines show the $1 \sigma$ uncertainty because of errors in the fitted parameters. The data used to plot the figure are from Basu and Mandel (2004)

to enhance the signature of the acoustic glitch caused by the He II ionisation zone and showed that the amplitude of the signal was a function of the $10.7-\mathrm{cm}$ flux, and that the amplitude decreased with increase in activity (see Fig. 30). The changes in the signal from the He II ionisation zone were also seen by Verner et al. (2006) in BiSON data and by Ballot et al. (2006b) in GOLF data. Like Basu and Mandel (2004), Verner et al. (2006) also found that the amplitude of the signal decreased as activity increased. Basu and Mandel (2004) interpreted the change in the ionisation zone as one caused by a change in temperature. Gough (2013a) however, challenged these results. He analysed the potential significance of the results of Basu and Mandel (2004), Verner et al. (2006) and Ballot et al. (2006b) under the assumption that the temporal variation of the amplitude of the He II signal is caused by a dilution by a broadly distributed magnetic field of the ionisation-induced influence on the wave propagation speed. He concluded that if the variation of the He II signal were a direct result of the presence of a temporally varying large-scale magnetic field, then the total solar cycle change of the spatial average of the magnetic field $\left\langle B^{2}\right\rangle$ in the vicinity of the He II ionisation zone is about $(30 \pm 10)^{2} \mathrm{Tesla}^{2}$ which is greater than most estimates. An examination of cycle 24 data should help in this regard.

Direct inversions of changes in the structure of the solar interior probed by the spherically symmetric global modes have yielded inconsistent results about changes inside the Sun. Dziembowski et al. (2000), looking at differences in mode frequencies between 1999.2 and 1996.4 claimed to detect a significant change at a depth of 25$100 \mathrm{Mm}$ which they interpreted as either being due to a magnetic perturbations of $(60 \mathrm{kG})^{2}$ or a relative temperature perturbation of about $1.2 \times 10^{-4}$. However, neither Basu (2002) nor Eff-Darwich et al. (2002) could find any measurable differences 
in the solar interior. Eff-Darwich et al. (2002) were able to put an upper limit of $3 \times 10^{-5}$ for any change in the sound speed at the base of the convection zone. Chou and Serebryanskiy (2005) and Serebryanskiy and Chou (2005) tried used a different technique-they plotted the smoothed scaled frequency differences as a function of the $w$ which is related to the lower turning point (Eq. 67), and showed that there was a discernible time-variation at $\log (w) \approx 2$.7. This corresponds approximately to the position of the convection-zone base. Chou and Serebryanskiy (2005) estimated the change to be caused by a magnetic field variation of $(1.7-1.9) \times 10^{5} \mathrm{G}$ at the convection-zone base assuming equipartition, or a temperature perturbation of about 44-132 K.

Baldner and Basu (2008) tried an altogether different approach. Instead of inverting data sets of different epochs and then comparing the results, they took 54 MDI data sets covering the period from 1996 May 1 to 2007 May 16, and 40 GONG data sets spanning 1995 May 7 to 2007 April 14 and did a principal component analysis (PCA) to separate the frequency differences over solar cycle 23 into a linear combination of different time-dependent components. They found that the dominant component was indeed predominantly a function of frequency, however, it also showed a small dependence on the lower turning point of the modes, implying a time dependent change in the interior. Baldner and Basu (2008) inverted the appropriately scaled first principal component to determine the sound-speed difference between solar minimum and maximum. They found a well-localised change of $\delta c^{2} / c^{2}=(7.23 \pm 2.08) \times 10^{-5}$ at $r=\left(0.712_{-0.0029}^{+0.0097}\right) R_{\odot}$. The change near the convection-zone base can be interpreted as a change in the magnetic field of about $290 \mathrm{kG}$. They also found changes in sound speed correlated with surface activity for $r>0.9 R_{\odot}$. Rabello-Soares (2012) examined the surface variations further by determining the frequencies of modes with $\ell \leq 900$ with MDI data centred around 2008 April (solar minimum) and 2002 May (solar maximum) and using those to resolve the surface structure better. She found a twolayer configuration - the sound speed at solar maximum was smaller than at solar minimum at round $5.5 \mathrm{Mm}$, the minimum-to-maximum difference decreased until $\sim 7 \mathrm{Mm}$. At larger depths, the sound speed at solar maximum was larger than at solar minimum and the difference increases with depth until $\sim 10 \mathrm{Mm}$. She could not detect deeper changes.

One of the interesting features of solar-cycle related frequency changes is the nature of the change in the frequencies of $\mathrm{f}$ modes. As mentioned in Sect. 3.3, the frequencies of $f$ modes are essentially independent of stratification and depend on the local value of gravitational acceleration $g$. Given that we do not expect the mass of the Sun to change as a function of the solar cycle, the f-mode changes led to investigations of changes in the solar "seismic radius". Dziembowski et al. (1998) looked into f-mode frequency changes in the period between 1996 May 1 and 1997 April 25 and arrived at a value of $4 \mathrm{~km}$. However, Goode et al. (1998) looked at the time variation of the f-mode frequencies between 1996 May 1 and 1997 April 25, to finding a relative radius difference of $6 \times 10^{-6}$ or $4 \mathrm{~km}$. They did not find any monotonic trend, instead they found a 1-year cycle in the frequencies, which of course is probably an artefact. Antia et al. (2000a) examined the f-mode frequency changes from 1995 to 1998 to conclude that if the changes were due to a change in radius, the change correspond to a change of $4.7 \mathrm{~km}$. 
The f mode changes are $\ell$ dependent. Antia et al. (2001b) looked into the $\ell$ dependence of the f-mode frequency changes over the period 1995-2000 and concluded that the most likely reason for the change is not a change in radius, but effects of magnetic fields. Sofia et al. (2005) however, argued that the $\ell$-dependent perturbations to f-mode frequencies points to a non-homologous change in the radius. Lefebvre et al. (2007) inverted the f-mode differences to determine the change in solar radius as a function of depth; their results showed a smaller change than was predicted by the Sofia et al. (2005) model but nonetheless showed a clear variation. Surprisingly, the issue has not been investigated using HMI data and for that matter reprocessed MDI data, and perhaps it is time that we do so!

\subsection{What do the even-order splittings tell us?}

The even-order frequency splittings and splitting coefficients change with time; even early helioseismic data showed so. Kuhn (1988a) had first suggested that the evenorder splittings showed a time variation; Libbrecht (1989), Libbrecht and Woodard (1990) and Woodard and Libbrecht (1993) agreed. Kuhn (1988b, 1989) and Libbrecht (1989) studied the temporal variation of the $a_{2}$ and $a_{4}$ coefficients and its relation to changes in the latitudinal dependence of limb temperature measurements, and Kuhn (1989) predicted that the same relationship should extend to higher order coefficients. The inference from the inversions of the BBSO even-order $a$ coefficient data carried out by Libbrecht and Woodard (1990) and Woodard and Libbrecht (1993) was that most of the variation in the even coefficients was localised close to the surface at the active latitudes, with a near-polar variation anti-correlated to the global activity level. Gough et al. (1993) reported latitude-dependent variations in $u$ between 1986 and 1989. Although they did not give a quantitative estimate, their figures suggest a change of a few parts in $10^{-4}$.

The temporal variations of the even- $a$ coefficients have been put on a much firmer basis with data collected by the GONG, MDI and HMI projects. In particular, one can show that the even $a$-coefficients are extremely well correlated with the corresponding Legendre component of the average magnetic field on the Sun (see e.g., Antia et al. 2001a). Howe et al. (2002) used the frequency changes of individual rotationally split components within an $(\ell, n)$ multiplet to carry out one-dimensional inversions for frequency shifts as a function of latitude; they showed that the frequency shifts were closely associated with regions of higher magnetic flux both as a function of time and latitude. There have been other attempts to determine whether the north-south symmetric latitudinal distribution of sound speed changes as a function of time. Antia et al. (2001a) did not find any time-variation at least below a radius of $0.98 R_{\odot}$ which is where the most reliable results are obtained. However, Baldner and Basu (2008) using a principal component analysis found significant differences in the speed between solar maximum and minimum at latitudes below $30^{\circ}$, their inversions were unstable at higher latitudes. They found an increase in the sound speed at the lower latitudes during solar maximum compared with solar minimum.

As mentioned earlier in Sect. 8.2, the even-order splitting coefficients can be interpreted as being either due to structural asphericities of a result of magnetic fields. 


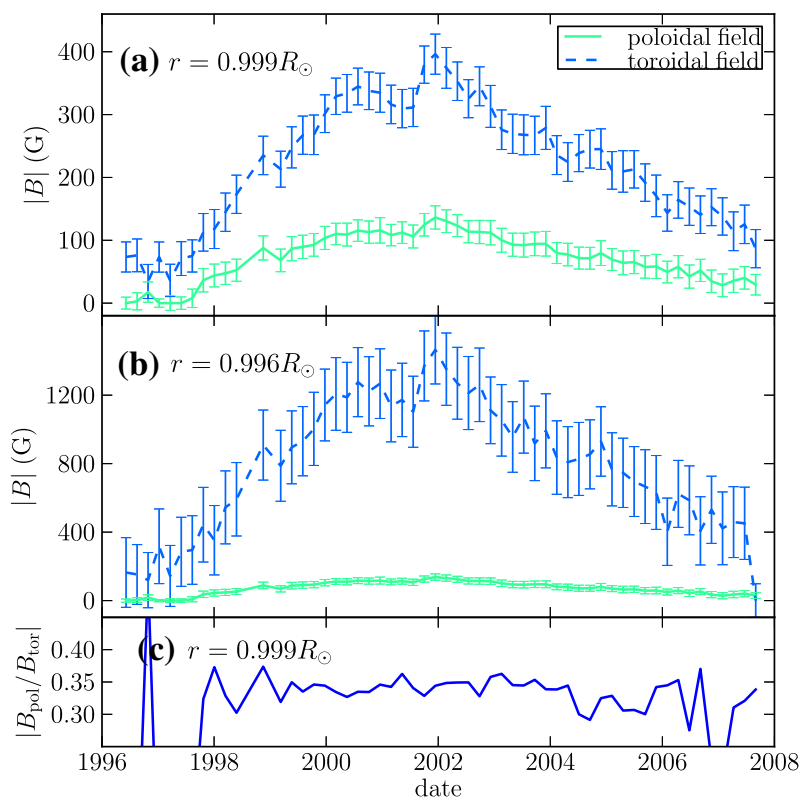

Fig. 31 Strength of the inferred magnetic fields plotted as a function of time for solar cycle 23. a, b The magnetic field strengths at $0.999 R_{\odot}$ and $0.996 R_{\odot}$ respectively. $\mathbf{c}$ The ratio of the poloidal to toroidal field at $0.999 R_{\odot}$, the ratio looks similar at $0.996 R_{\odot}$. Thus while the strength of both poloidal and toroidal fields change with time, their ratio does not evolve much. The results in this figure are from Baldner et al. (2009). (Image courtesy of Charles Baldner)

Baldner et al. (2009) analysed the changes in these coefficients in terms of magnetic fields. They found at that each epoch, they could explain the data as being due to a combination of a poloidal and a near-surface toroidal field, with a changing strength (see Fig. 31). They also found that the strengths of both components tracked the 10.7$\mathrm{cm}$ radio flux, however, there were hints of saturation at high activity (see Fig. 32). They also found that the relation between the magnetic field strengths during the rising phase was different from that of the falling phase.

\subsection{Changes in solar dynamics}

The most visible solar-cycle changes revealed by global helioseismic data are changes in solar dynamics. Readers are again referred to the Living Reviews in Solar Physics article by Howe (2009) for details, we describe a few highlights.

The most visible change in solar dynamics are changes in the "zonal flows" which are alternating bands of prograde and retrograde motion that can be observed when the time-average rotation rate is subtracted from the rotation rate at any one epoch (e.g., Kosovichev and Schou 1997; Schou 1999; Antia and Basu 2000, 2001, 2010, 2013; Howe et al. 2000, 2009, 2011, 2013a, b), i.e.,

$$
\delta \Omega(r, \theta, t)=\Omega(r, \theta, t)-\langle\Omega(r, \theta, t)\rangle,
$$




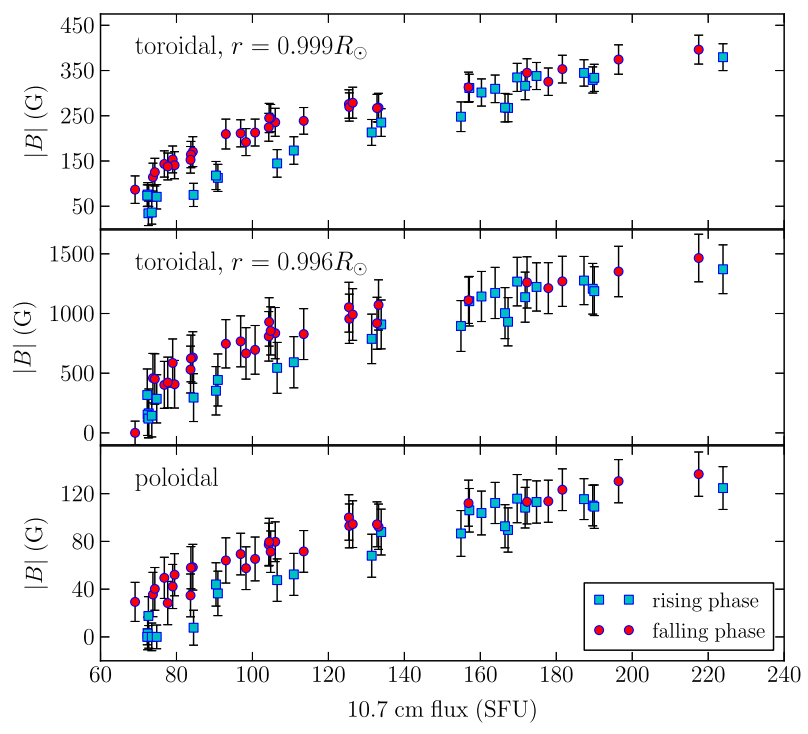

Fig. 32 The strength of the solar interior magnetic field during cycle 23 plotted as a function of the 10.7-cm flux. The top two panels show the toroidal field at $0.999 R_{\odot}$ and $0.996 R_{\odot}$. The lowest panel is the poloidal field. The cyan squares are for the rising phase of the cycle while the magenta circles are for the falling phase. Results are from Baldner et al. (2009). (Image courtesy of Charles Baldner)

where $\Omega$ is the rotation rate, $r$, the radius, $\theta$ the latitude and $t$ time. $\langle\Omega(r, \theta, t)\rangle$ is the time average of the rotation rate. From the zonal flow rate one can define the zonal flow velocity as

$$
\delta v_{\phi}=\delta \Omega r \cos \theta .
$$

The zonal flows are thought to be the same as the "torsional oscillations" seen at the solar surface (Howard and Labonte 1980; Ulrich et al. 1988; Snodgrass 1992). At low latitudes, just like the torsional oscillations, the zonal-flow bands appear to migrate towards the equator with time. At high latitudes these move polewards with time (Antia and Basu 2001; Ulrich 2001). As more and more data have been collected, it has become clear that the zonal flows penetrate through most of the solar convection zone (Vorontsov et al. 2002; Basu and Antia 2003; Howe et al. 2006b; Antia et al. 2008). At low latitudes, zonal flows seem to rise from deeper in the convection zone towards the surface as a function of time. We show the changing nature of solar zonal flows in Figs. 33 and 34.

While the most visible changes in solar dynamics are in the zonal flows, and which are highly latitudinally dependent, there are also changes in the component of the rotation that is independent of latitude. We can see this by inverting just the $a_{1}$ coefficient. In Fig. 35, we show the changes in the latitude-independent part of the solar rotation in the outer layers of the Sun as determined using MDI data. The results were obtained by subtracting the time-averaged $a_{1}$ inversion result from the $a_{1}$ result at any given epoch. Note that there appears to be a solar-cycle related change, with the fastest rotation at the cycle 23 solar maximum and the slowest at the two minima. Rotation during cycle 24 also appears to be somewhat slower than that during cycle 23, at least 
Fig. 33 The zonal flow velocity at $0.98 R_{\odot}$ as determined using GONG data plotted as a function of latitude and time. The top panel was obtained by subtracting the average of the cycle 23 rotation velocities, while the lower panel is that obtained by subtracting the cycle 24 average rotation between 2009 and 2012. (Figure from Antia and Basu 2013)
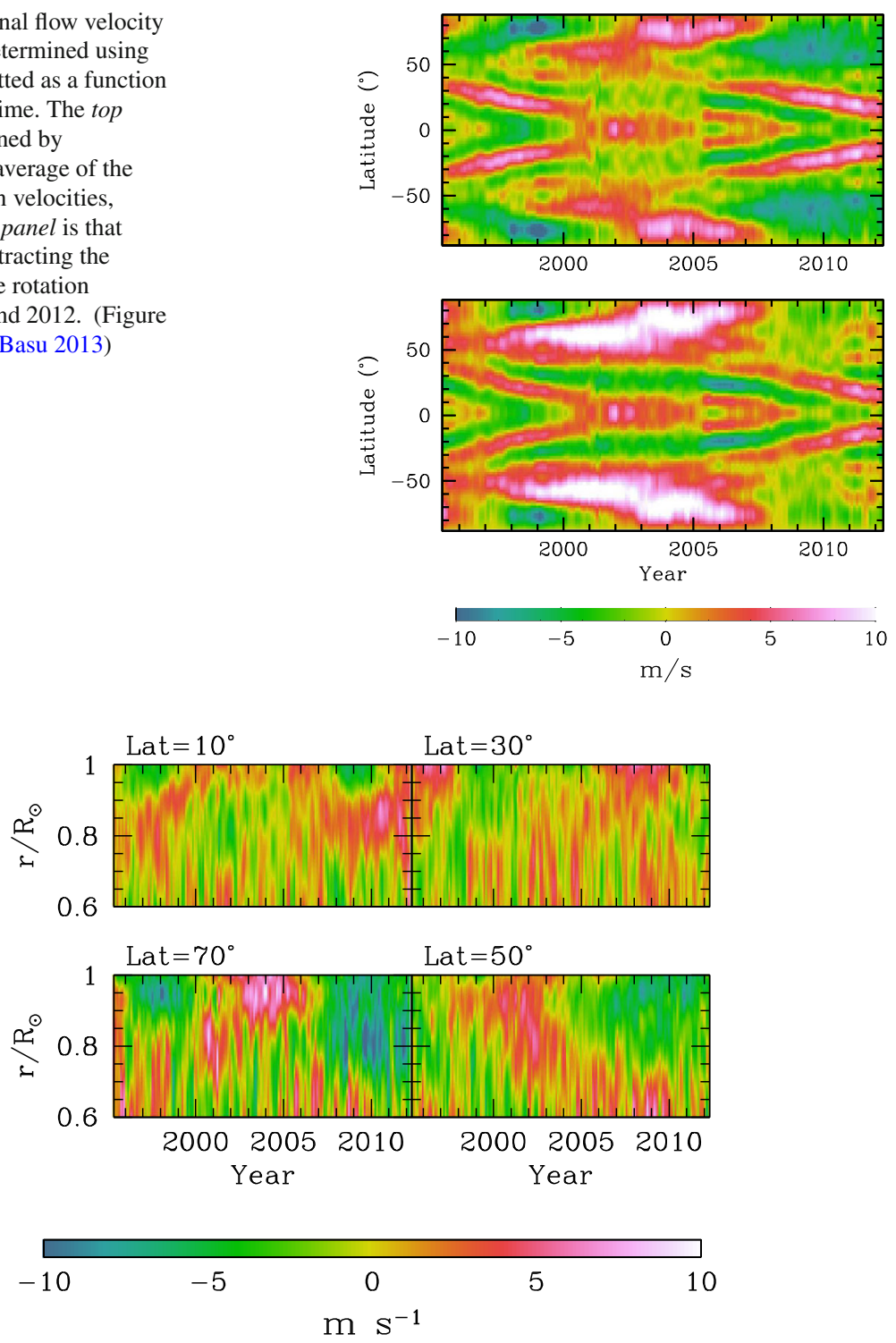

Fig. 34 The zonal flow velocity plotted as a function of radius and time for a few latitudes. Results were obtained using data obtained by the GONG project. (Figure from Antia and Basu 2013)

in the outer layers. The results for deeper layers are too noisy to draw any conclusion. There is significant uncertainty as to whether the dynamics of the solar radiative zone shows any time dependence (Eff-Darwich and Korzennik 2013).

While the dynamics within the convection zone clearly changes with time, the tachocline does not show much change. Antia and Basu (2011) did a detailed analysis of the then available GONG and MDI data and found that of the various properties of 
Fig. 35 The change in the latitudinally independent component of the solar rotation rate plotted as a function of time. (Figure from Antia and Basu 2013)

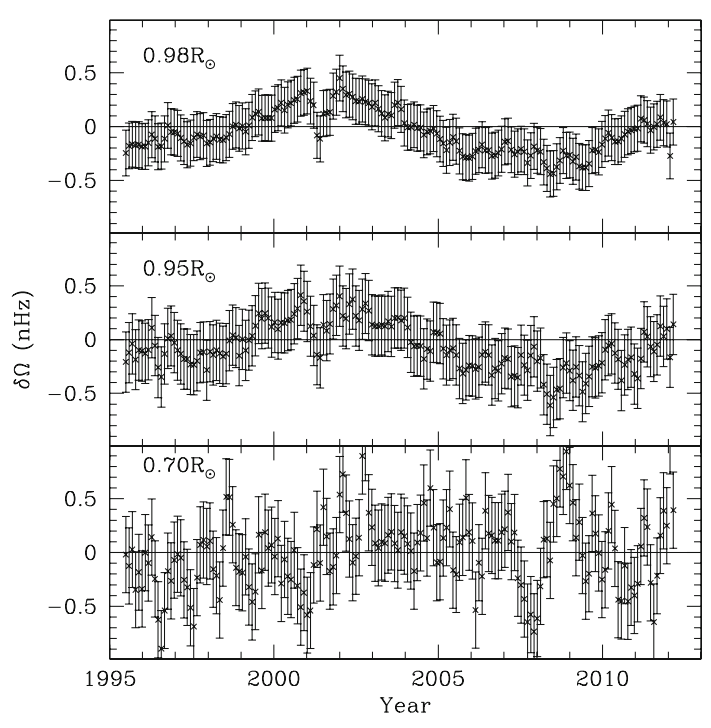

the tachocline, the jump in the rotation rate across the tachocline is the only parameter that shows a significant change with solar activity, but even that change is small.

\subsection{The "peculiar" solar cycle 24}

Solar cycle 24 is showing all signs of being a very weak cycle. The minimum that preceded it was extremely long and very quiet and unusual in its depth-it had more sunspot free days and any recorded in the space age, and the 10.7-cm flux was the lowest ever recorded. The polar fields observed by the Wilcox solar Observatory and other instruments were also very weak (e.g., Lo et al. 2010; Gopalswamy et al. 2012). The structure of the solar corona was very different during the minimum-instead of having a simple, equatorial configuration as is normally seen during solar minima, the corona was bright at higher latitudes too. Another noteworthy feature is that the fast solar winds were confined to higher latitudes (Manoharan 2012). In fact the minimum was unusual enough to have multiple workshops dedicated to it and many of the measured peculiarities are discussed in the workshop proceedings edited by Cranmer et al. (2010).

Of course one could ask if the minimum was really peculiar, and it is possible that the lack of extensive data for earlier solar cycles is fooling us. After all, the minimum between cycles 23 and 24 was the not the only one with many sunspot free days, there were sunspot free minima in the early part of the twentieth century (the minima of 1900 and 1910). Going even further back in time, we can find a lot of other minima that were as deep at the cycle 24 minimum, at least as far as sunspot numbers are concerned (see, e.g., Sheeley 2010; Basu 2013). As mentioned earlier, a peculiarity of the minimum was the configuration of the solar corona. However, as Judge et al. (2010) have pointed out, there are eclipse records that show that the solar corona during the solar minimum 


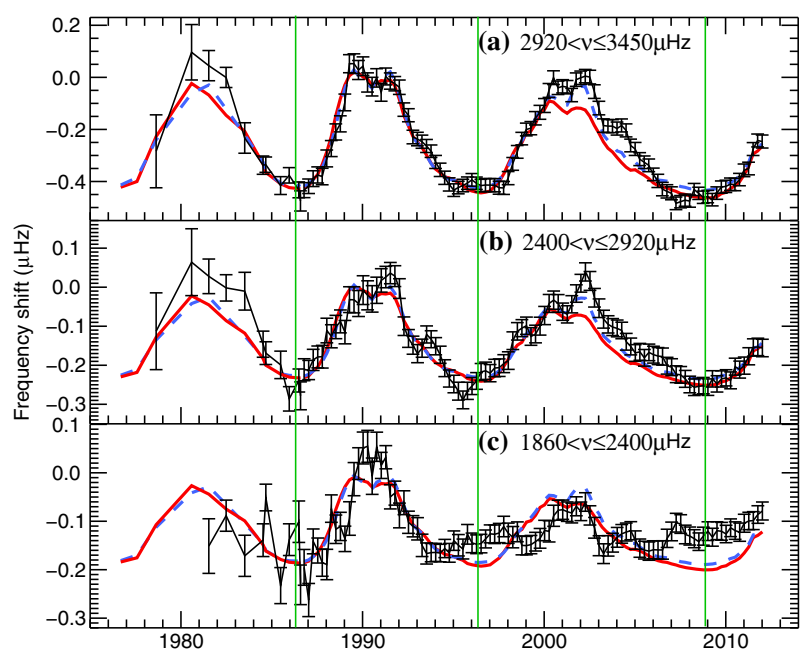

Fig. 36 Average frequency shifts of solar oscillations observed by BiSON as a function of time for three different frequency ranges. The plotted shifts were calculated with respect to the averaged frequencies during the cycle 22 maximum, specifically over the period 1988 October to 1992 April. The black points in each panel show the average shift in frequency. The blue dashes show the 10.7-cm flux and the red dotdashed line is the International Sunspot Number. The green vertical lines mark the approximate positions of solar minima. (Image courtesy of Anne-Marie Broomhall)

circa 1901 was very similar to that of 2009 as seen in eclipse photographs. Thus the seeming peculiarity of the solar minimum is simply a result of not having enough data; after all the Sun is 4.57 Gyr old, the sunspot data go back to only about 400 years and solar magnetic field data to date back to only 40 years.

While whether or not cycle 24 itself is "peculiar" is a matter of definition, it is certainly different from the cycles seen over the last 60 or so years for which we have had more data than just the sunspot number. Unlike earlier candidates for possibly peculiar cycles, we have detailed helioseismic data for cycle 24 which allow us to compare the internal structure and dynamics of cycle 24 with that of cycle 23 , and the indications are that the Sun, particularly solar dynamics, has a different behaviour in cycle 24 compared to cycle 23 . As mentioned earlier, the latitudinally independent part of solar rotation appears to be slower in cycle 24 compared to the cycle 23. Details of the solar zonal flows are different too, as can be seen from Figs. 33 and 34 and Antia and Basu (2013) had found that while the zonal flows at all latitudes in cycle 23 can be fitted with three harmonics of an 11.7 year period, one cannot simultaneously fit the mid-latitudes of cycle 24 .

There were early signs that the Sun was heading towards an unusual phase, unfortunately, we missed a chance to predict the peculiarity and only saw the signs after the onset of cycle 24 (Basu et al. 2012a). BiSON has been collecting data for over 30 years and its observations cover cycles 22 and 23 in their entirely and all of cycle 24 to date. They also cover a few epochs of cycle 21. Basu et al. (2012a) found that the frequency-dependence of the solar-cycle related shifts were different in cycle 23 compared with cycle 21 . This can be seen in Fig. 36 where we plot the frequency shifts of 
Fig. 37 Frequency shifts in the mid-frequency range (upper panel) and low-frequency range (lower panel) plotted as a function of the frequency shifts in the high-frequency range for cycles 22,23 , and 24 . The frequency shifts have been smoothed to remove the signature of the 2-year cycle. The frequency ranges are identical to those in Fig. 36. (Image courtesy of Anne-Marie Broomhall)

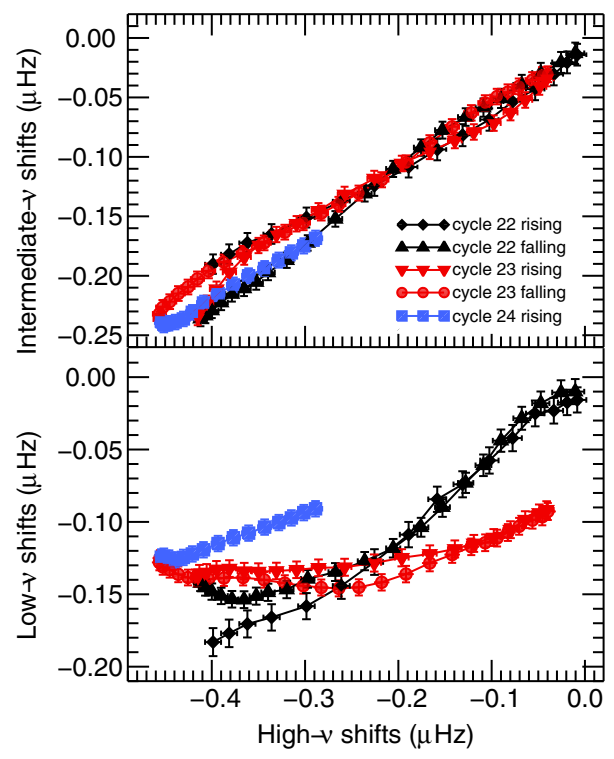

low-degree solar modes for three different frequency bands. The shifts are calculated with respect to the frequencies at the maximum of cycle 22. Note that the high- and intermediate-frequency bands show a different pattern of the shifts compared with that of the low-frequency band. The difference is particularly notable for cycle 23. Also note that the frequency shifts in the high- and intermediate-frequency bands stop following the sunspot number (in red) and the 10.7-cm flux (in blue dashes) sometime just before the maximum of cycle 23. This figure thus shows that cycle 23 itself was quite different from cycle 22, and perhaps the "peculiarity" of cycle 24 had its seeds in the descending phase of the previous cycle.

Another way to show the differences between the cycles is to plot the shifts in the different frequency ranges against one another, as has been done in Fig. 37. Note that the shifts of the intermediate frequency range appears to follow that of the high frequency range for all cycles shown. The situation is different for the low-frequency modes. Cycle 23 is very different, and although cycle 24 the low-frequency shifts in cycle 24 have the same slope with respect to the cycle 22 shifts, they are following a different path altogether. It is therefore quite possible that as far as the signatures from the solar interior are concerned, each cycle is distinct in it own way and that the assumption that we can have a "typical" solar cycle is simply a reflection of the lack of data.

\section{The question of mode excitation}

All the results discussed in the earlier sections were obtained by analysing and interpreting data on the frequencies of solar oscillation. However, the frequencies are not the only observable - the height of the individual modes as well as the width of the 
peaks in the power spectra can be measured as well. Using the information contained within these observations requires us to understand how modes are excited and damped. The two possibilities for exciting the modes are (1) self excitation and (2) stochastic excitation by turbulent convection of intrinsically damped oscillations.

In the solar case when self-excitation due to over-stability ${ }^{1}$ was considered, it was argued that the limiting amplitude could be determined by non-linear processes such as mode coupling (e.g., Vandakurov 1979; Dziembowski 1982). Ando and Osaki (1975) performed one of the first calculations to determine whether or not solar modes are unstable, and found them to be so. They found that the driving of oscillations is mainly due to the $\kappa$ mechanism (Cox 1967) of the hydrogen ionisation zone with damping being provided by radiative loses in the optically thin layers. However, their results ignored the coupling of convection to pulsations. Similar results were obtained by Goldreich and Keeley (1977b) who found that if damping by turbulent viscosity was neglected, all modes with periods greater than about 6 min were unstable, and that the $\kappa$ mechanism was responsible for driving the modes. Modes with shorter periods were stabilised by radiative damping. However, when they included a model (albeit a crude one) of the interaction between convection and oscillations, they found that the turbulent dissipation of pulsational energy made all the modes stable. There have been many investigations to determine whether or not solar modes are stable (e.g., Gough 1980; Christensen-Dalsgaard and Frandsen 1983; Antia et al. 1982, 1988), the results have been contradictory and have depended on details of how energy dissipation was treated. Goldreich and Keeley (1977b) had concluded that the lack of a reliable timedependent theory of convection the process of determining the stability of solar modes an uncertain one.

The consensus today is that solar oscillation modes are stochastically excited by turbulent convection. Goldreich and Keeley (1977a) argued that the small line-widths and low amplitudes of low-order p modes makes it hard to imagine non-linear processes that lead to the saturation of unstable modes at the observed low amplitudes. If the modes are accepted as being stable, then the source of excitation becomes the question. Guided by general principles of thermodynamics that indicate that sources of excitation are intimately related to the sources of damping, and since turbulent viscosity appears to be one of the main damping sources of p modes, the question is how they might act as sources of excitation. Goldreich and Keeley (1977c) presented one of the first formulations of for the stochastic excitation of solar $\mathrm{p}$ modes. Although they were unable to reproduce the observed $\mathrm{p}$ mode velocity amplitudes at that time, the idea behind the work has remained the basis for other calculations. Under this assumption, the oscillations can be described as a damped oscillator whose amplitude $A$ satisfies the equation

$$
\frac{\mathrm{d}^{2} A}{\mathrm{~d} t^{2}}+2 \eta \frac{\mathrm{d} A}{\mathrm{~d} t}+\omega_{0}^{2} A=f(t),
$$

\footnotetext{
1 'Stable' modes are not self-excited. When it comes to excitation, terms 'unstable' and 'overstable' are often used interchangeably, but they have a subtle difference. Unstable modes are those whose amplitudes grow exponentially with time. Overstable modes are a special class of unstable modes and correspond to harmonic oscillations, i.e., they are periodic, and the amplitudes of these oscillations increase with time.
} 
where $\eta$ is the damping constant, and $f(t)$ is a random forcing function that is independent of $A$. The complexity of the model lies in determining the damping term $\eta$ and the forcing function $f$. These depend on entropy fluctuations and fluctuations of the Reynolds stress produced by convection in stellar envelopes.

A tremendous amount of work has been done since the Goldreich-Keeley papers with regards to mode excitation in the Sun. Gough (1980) showed that turbulentexcitation models predict the right order of magnitude for the p-mode amplitudes and also explains the excitation of many modes simultaneously. Kumar et al. (1988) calculated how the mode energy should be distributed, Goldreich and Kumar (1988) derived expressions for the emissivity and absorptivity of acoustic radiation by low Mach number turbulent fluids, and Goldreich et al. (1994) investigated the rates at which energy is supplied to modes as a function of their frequencies and degree. The stochastic excitation model has also been applied to solar-like oscillations in other stars Sun-like stars (Christensen-Dalsgaard and Frandsen 1983; Balmforth 1992; Houdek et al. 1999; Dupret et al. 2009; Samadi and Goupil 2001; Belkacem and Samadi 2013, etc.)

Observations do guide us somewhat. For instance, even within the rubric of stochastic mode excitation there was a question as to whether the convective flux perturbations that are induced by oscillations play a role. Christensen-Dalsgaard et al. (1989a) used the then available data on line-widths and showed that calculations that took some account of the perturbation of convective fluxes fared much better when compared with observations than calculations that did not. The data Christensen-Dalsgaard et al. (1989a) used for their tests were crude by today's standards, but they were able to demonstrate the diagnostic potential of p-mode line widths. Elsworth et al. (1995b) and Chaplin et al. (1995) looked into the distribution energy in the modes, and found the energy distribution followed a negative exponential form. These results agree with the prediction of Kumar et al. (1988). However, both Elsworth et al. (1995b) and Chaplin et al. (1995) found some high-energy events that did not follow the trend. Chaplin et al. (1997) did an analysis of mode linewidths using BiSON data and found that the linewidths predicted by theory (in particular Goldreich and Murray 1994; Balmforth 1992) were in line with the BiSON results.

Another question that could be asked about the excitation mechanism is exactly where the excitation sources lie. Goode et al. (1992) analysed high- $\ell$ data to show that the likely source of excitation are 'exploding granules' about $200 \mathrm{~m}$ below the base of the photosphere. Kumar and Lu (1991) looked above the acoustic cut-off frequency and place the sources somewhat $\operatorname{lower}$, at $\log (P)=5.7$, deeper than what would be suggested by the mixing length approximation. The Goode et al. results were based on the mixing length approximation that postulates that upflows and downflows are symmetric, simulations of convection have shown that this is not the case at all, and in fact that the downflows are fast and cold and are what are observed as intergranular lanes, and that upflows are slow and hot. Observations now suggest that the sources of excitation are not exploding granules, instead they are located in the dark intergranular lanes (Rimmele et al. 1995) and are related to the collapse of these lanes (Goode et al. 1999).

One of the biggest obstacles in calculated mode-excitation related properties of the Sun and Sun-like stars is the lack of a good description of convection. This makes using 
the information in mode amplitudes and mode widths difficult to use. The conventional mixing length approximation is clearly wrong when compared with simulations, even variations such as that of Canuto and Mazzitelli (1991) do not describe the dynamics of convection. Non-local formalisms, even those that include the anisotropy of flows, have been proposed (e.g., Gough 1977a,b), but all such formalisms have at least one, and sometimes more, free parameters and thus lack true predictive power. As a result, it is becoming increasingly common to use simulations of stellar convection to perform such calculations. Stein and Nordlund (2001) first derived expressions for the excitation of radial modes. Other works, such as those of Samadi et al. (2003a, b, 2007), Stein et al. (2004), etc., have followed. Stein et al. (2004) for instance have shown that they can reproduce the excitation rates of modes of $\ell=0-3$. They have also applied their method to a few other stars. Given the difficulty in modelling convection analytically, we expect that the study of mode excitation in simulations of convection to become the usual way will study mode-excitation in solar-like oscillators.

\section{Seismology of other stars}

Pulsating stars, such as Cepheids and RR Lyræ stars have been known for a long time. These stars lie on the instability strip and their pulsations are excited by a "heat engine" such as the $\kappa$-mechanism (see e.g., Cox 1967). We shall not discuss these stars here. This section is devoted to solar-like oscillations in stars, i.e., oscillations in stars that, like in the case of the Sun, are excited stochastically by a star's convective envelope. For recent reviews of this subject, readers are referred to Chaplin and Miglio (2013), Christensen-Dalsgaard (2016), etc.

There are three major differences between the study of the Sun and other stars using solar-like oscillations: The first difference is related to how stars are observed. Resolved-disc observations of stars other than the Sun are not possible. This means the data sets for other stars are restricted to modes of low degree only. We had seen earlier the change in any scalar quantity can be written in terms of spherical harmonics and a oscillatory function, as in Eqs. (33) and (34). We can express the intensity of a mode at the observing surface in the same manner. Since we are looking at a physical quantity, we use only the real part, thus,

$$
I(\theta, \phi, t) \propto \Re\left\{Y_{\ell}^{m}(\theta, \phi) \exp \left[-i\left(\omega t+\delta_{0}\right)\right]\right\}
$$

where, $I$ is the intensity and $\delta_{0}$ is a phase. In other words

$$
I(\theta, \phi, t) \propto P_{\ell}^{m}(\cos \theta) \cos \left(m \phi-\omega t+\delta_{0}\right) .
$$

We need to integrate $I(\theta, \phi, t)$ over the visible disc in order to obtain the result of whole-disc observations. Neglecting the effects of limb darkening, the integrated intensity is

$$
I(t)=\frac{1}{A} \int I(\theta, \phi, t) \mathrm{d} A,
$$


where $A$ denotes the area of the visible surface. If we have a spherically symmetric system, we are free to choose our coordinate system. For ease of calculation we assume that the polar axis is pointed towards the observer which makes the integral 0 for all $m$ except $m=0$, and for $m=0$

$$
I(t)=\mathcal{S}_{\ell} I_{0} \cos \left(\omega t-\delta_{0}\right)
$$

where, $S_{\ell}$ is the spatial response function given by

$$
\begin{aligned}
\mathcal{S}_{\ell} & =\frac{1}{\pi} \int_{0}^{2 \pi} \mathrm{d} \phi \int_{0}^{\pi / 2} \sqrt{2 \ell+1} P_{\ell}(\cos \theta) \cos \theta \sin \theta \mathrm{d} \theta \\
& =2 \sqrt{2 \ell+1} \int_{0}^{\pi / 2} P_{\ell}(\cos \theta) \cos \theta \sin \theta \mathrm{d} \theta
\end{aligned}
$$

On evaluating the integral for different values of $\ell$ one finds that $\mathcal{S}_{\ell}$ which controls the amplitude of the signal, becomes small quite rapidly as $\ell$ is increased, and thus only very low $\ell$ modes can be observed (see Christensen-Dalsgaard and Gough 1982). The second difference between helioseismic and asteroseismic analyses is that unlike the Sun, we generally do not have independent estimates of the mass, radius, luminosity and age of the stars, and all these quantities have to be determined from the seismic data. The third difference is that stars come in many different masses and radii and are at different states of evolution, and as a result, the frequency spectrum of the stars can often be much more complicated than that of the Sun.

As had been mentioned earlier in Sect. 3.3, the propagation of modes of different frequencies in star is controlled by the Lamb and Brunt-Väisälä frequencies. For a given mode, the profiles of these two frequencies determine what type of a mode it is and where it can propagate. The propagation diagram for stars in different stages of evolution can be very different from each other because of the how the Brunt-Väisälä frequency evolves with time. In Fig. 38, we show the propagation diagram for a $1 M_{\odot}$ model at three stages of evolution - the main sequence, the subgiant, and the red giant. Note how the buoyancy frequency gets larger and larger towards the core as the star evolves.

In un-evolved stars like the Sun, $\mathrm{p}$ - and g-modes occupy distinct oscillation cavities and occur in distinct frequency regimes. The frequency regimes are not so distinct in evolved stars where the two cavities are in close proximity (see Fig. 38) and hence, the cavities can couple. This results in modes that have p-mode like character in the outer layers and g-mode like character in the interior with a small evanescent region in between. These are the so-called 'mixed modes'. The widely observed pure p modes have their highest amplitudes close to the surface, and hence carry less information about the core. Mixed modes, on the other hand, have high amplitudes in the core (just like $g$ modes), but also have high amplitudes at the surface (making them observable). This dual nature of mixed modes make them ideal for studying stellar cores. Note that since there are cannot be $\ell=0 \mathrm{~g}$ modes, there can be no $\ell=0$ mixed modes. Mixed modes are seen in subgiants. Most subgiant mixed modes are $\ell=1$ modes, though some $\ell=2$ mixed modes are also seen The pulsation spectrum of red-giants on the 
Fig. 38 Propagation diagram for a $1 M_{\odot}$ stellar model in the main-sequence (a), subgiant (b) and redgiant (c) stages. The blue line is the Brunt-Väisälä frequency, while the red lines show the Lamb frequency for $\ell=1$ (solid line), $\ell=5$ (small dash), $\ell=20$ (long dash) and $\ell=100$ (dot-dashed line). The horizontal cyan line shows the propagation cavity for an $\ell=1$, $1 \mathrm{mHz}$ mode and the horizontal dotted magenta line shows the cavity for an $\ell=1,0.5 \mathrm{mHz}$ mode. Note that for the star in a, the two modes can only exists as $\mathrm{p}$ modes with a cavity in the outer part of the star. In $\mathbf{b}$ and $\mathbf{c}$ each mode has two cavities where they can propagate. The region in between is where the modes are evanescent. Note that in $\mathbf{b}$ and $\mathbf{c}$ the evanescent zones for the modes are small and hence the interior and exterior cavities can couple giving rise to a mixed modes

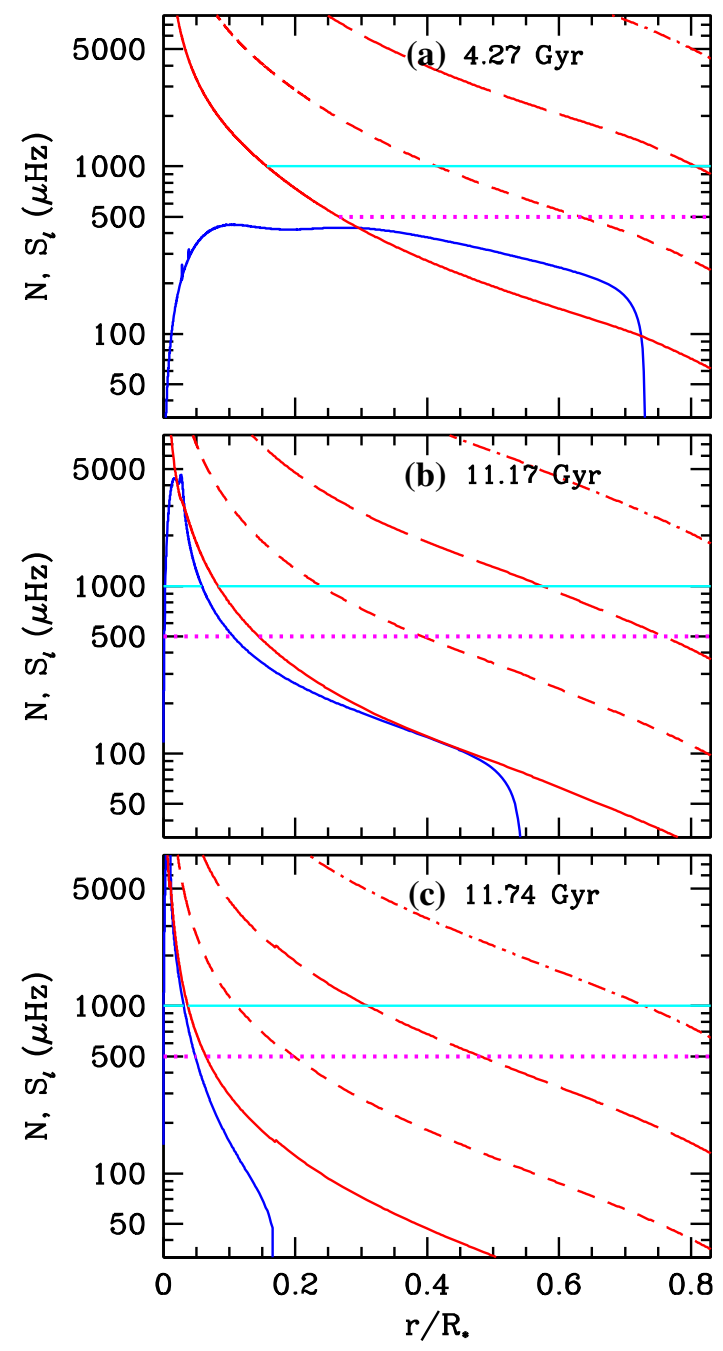

other hand are extremely complicated. Almost all non-radial modes have g-mode like character in the core and p-mode like character in the envelope, however, only a few of all the possible modes are excited. The excited modes are those with the lowest mode inertia (Dupret et al. 2009).

The frequency of an observed mode is not sufficient to tell us whether or not it is a mixed mode. Its frequency needs to be examined in the context of the frequencies of its neighbouring modes, and in particular, the frequency separation between neighbouring modes. For this we make use of the fact that $\mathrm{p}$ modes are almost equally spaced in frequency, and that $\ell=0$ and 2 modes have similar frequencies [Eq. (68)], as do $\ell=1$ and 3 modes. The usual practice is to divide the observed power spectrum (or frequencies) of a star into frequency slices of width $\Delta v$ and stack the slices on top of each other. The result is usually called an 'échelle' diagram (Grec et al. 1983). If 

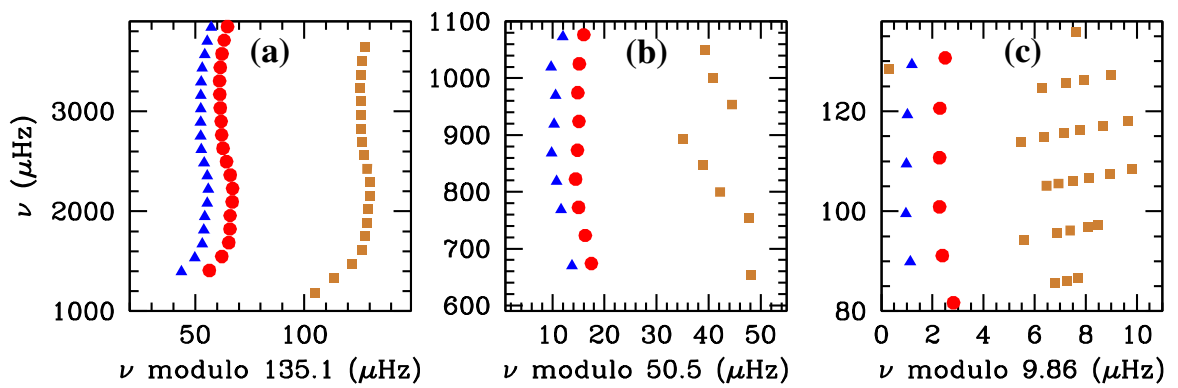

Fig. 39 The frequencies of three stars shown in the form of an 'Échelle' diagram. Échelle diagrams are plotted by dividing the observed power spectrum (or frequencies) of a star into frequency slices of width $\Delta v$ and stacking the slices on top of each other. Red circles are $\ell=0$ modes, brown squares are $\ell=1$ and blue triangles are $\ell=2$ modes. a The Sun, $\mathbf{b}$ a subgiant and $\mathbf{c}$ a red giant. Note that for the Sun, a main sequence star, all the modes of a given degree line up. In the case of the subgiant, the $\ell=1$ modes show a break. This break is an "avoided crossing" and is a signature of the presence of mixed modes. Note the large number of $\ell=1$ modes in the red giant. These are all mixed modes and the frequencies of these modes depend on details of the buoyancy frequency profile

the $\Delta v$ used is correct, the $\ell=0$ modes line up, with the $\ell=2$ modes close by, the same happens to $\ell=1$ and 3 modes. If an avoided crossing is present, it shows up as a deviation in the vertical structure of the modes of that degree in the échelle diagram. We show the échelle diagram of three stars in Fig. 39, note that for the Sun (Panel a) all modes line up and for the subgiant (panel b) the $\ell=1$ modes show a break, and the giant (panel c) shows many $\ell=1$ mixed modes.

\subsection{Asteroseismic analyses}

How asteroseismic data are analysed depends on the type of data available. For stars with good signal-to-noise characteristics and long time series (just how long depends on the star in question), individual peaks in the oscillation spectra can be resolved. We can determine a set of frequencies from each star. For such stars, the usual way of analysis to construct stellar models, calculate their frequencies and see which model fits the data. This is described further in Sect. 11.1.2.

Estimates of frequencies of different modes are, however, available only for a handful of stars. For most stars it is easier to determine two average asteroseismic properties, the large frequency separation $\Delta v$ (Eq. 68) and the frequency of maximum power $v_{\max }$. As mentioned earlier, $\Delta v$ scales as the square-root of density (Ulrich 1986; Christensen-Dalsgaard 1988), thus

$$
\frac{\Delta v}{\Delta v_{\odot}} \simeq \sqrt{\frac{M / M_{\odot}}{\left(R / R_{\odot}\right)^{3}}}
$$

Many methods have been developed to determine $\Delta v$ even when the modes are not resolved (see e.g., Chaplin et al. 2008; Stello et al. 2009; Hekker et al. 2011). 


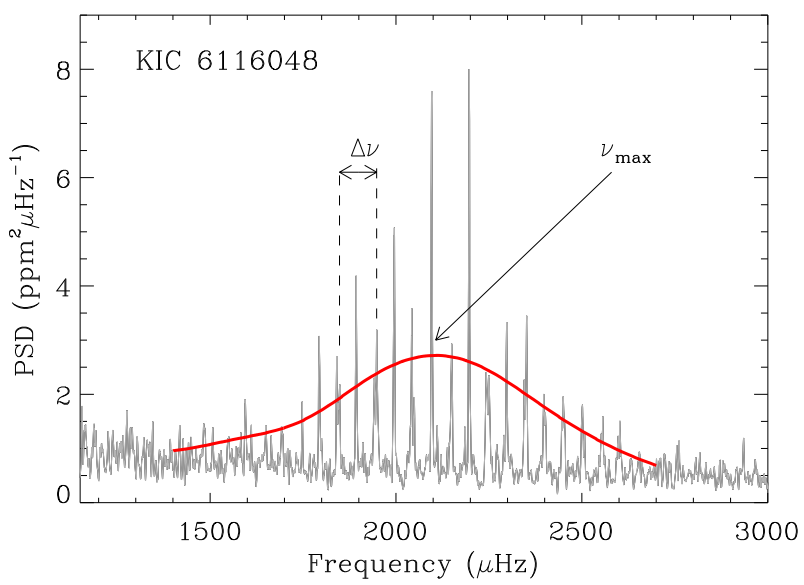

Fig. 40 The oscillation power spectrum of the star KIC 6116048 computed with Kepler data obtained over a period of 1 month. The red line is the envelope of power obtained by heavily smoothing the power spectrum. The envelope has been multiplied by a factor of 5 to make it more visible. (Image courtesy of William J. Chaplin)

The mode amplitude (and power) are modulated by an envelope that is roughly Gaussian in shape (red curve in Fig. 40) in stars (including the Sun) for which the solar-like oscillations have been observed. The maximum of this envelope is $v_{\max }$. The frequency at which power is the maximum is $v_{\max } \cdot v_{\max }$ is related to the acoustic cut-off frequency of a star (e.g., Kjeldsen and Bedding 1995; Bedding and Kjeldsen 2003) and scales as

$$
\frac{v_{\max }}{v_{\max , \odot}} \simeq \frac{M / M_{\odot}}{\left(R / R_{\odot}\right)^{2} \sqrt{\left(T_{\mathrm{eff}} / T_{\mathrm{eff}, \odot}\right)}} .
$$

Again, $v_{\max }$ can be measure in low $\mathrm{S} / \mathrm{N}$ spectra. $v_{\max }$ is an intriguing observable, and unlike the case of $\Delta v$, we do not as yet have a theory explaining the quantity. There are some studies in this regard (e.g., see Belkacem et al. 2011), but the issue has not been resolved. $v_{\max }$ carries diagnostic information on the excitation and damping of stellar modes, and hence must depend on the physical conditions in the near-surface layers where the modes are excited. Close to the surface, the behaviour of the waves is influenced by the acoustic cut-off frequency (Eq. 80). Brown et al. (1991) argued that $v_{\max } \propto v_{\mathrm{ac}}\left(\nu_{\mathrm{ac}} \equiv \omega_{\mathrm{ac}} / 2 \pi\right)$ because both frequencies are determined by conditions in the near surface layers. Kjeldsen and Bedding (1995) turned this into a relation linking $v_{\max }$ to near-surface properties by noting that Eq. (80) can be approximated as $v_{\mathrm{ac}} \propto g T_{\mathrm{eff}}^{1 / 2}$ and the relation in Eq. (171) follows from this.

There have been numerous tests of the $v_{\max }$ scaling relation. Bruntt et al. (2010) and Bedding (2014) compared asteroseismic and independently determined properties (e.g., from binaries) of a selection of bright solar-type stars and red giants. All the stars showed solar-like oscillations from ground-based or CoRoT observations. The estimated properties were found to agree at the level of precision of the uncertainties, i.e., to $10 \%$ or better. The comparisons were extended to stars observed by Kepler. Silva Aguirre et al. (2012) used asteroseismic data on 22 of the brightest Kepler targets 
with detected solar-like oscillations and found agreement between stellar radii inferred from the scaling relations and those inferred from using Hipparcos parallaxes at the level of a few percent. Huber et al. (2012) combined interferometric observations of some of the brightest Kepler and CoRoT targets with Hipparcos parallaxes and also found excellent agreement, at the $5 \%$ level, with the stellar radii inferred using the scaling relation. There have been other, more indirect, tests of the relation. For instance, Silva Aguirre et al. (2015) determined the properties of 33 Kepler objects of interest (i.e., Kepler targets suspected to have an exoplanetary system) obtained from using combinations of individual frequencies of those stars, as well as from using the $\Delta v$ and $v_{\max }$ only (but through grid-based analyses with $\Delta v$ calculated from frequencies) and the agreement is very good, except in the case of three outliers. For the rest, the median fractional difference and standard deviation between the two estimates are $0.5 \pm 1.2 \%$ for density, $0.7 \pm 0.8 \%$ for radius and $1.8 \pm 2.1 \%$ for mass. More recently Coelho et al. (2015) tested the $v_{\max }$ scaling relation for dwarfs and subgiants and ruled out departures from the $g T_{\text {eff }}^{-1 / 2}$ scaling at the level of $\simeq 1.5 \%$ over the full $1560 \mathrm{~K}$ range in $T_{\text {eff }}$ that was tested. There is some uncertainty over the absolute calibration of the scaling, but again of the same order.

\subsubsection{Grid based modelling}

Grid based modelling is more correctly a grid based search to determine stellar parameters. When $\Delta v, v_{\max }$ and $T_{\text {eff }}$ are known, Eqs. (170) and (171) represents two equations in two unknowns ( $M$ and $R$ ), and can be solved to obtain $M, R$ (and hence surface gravity $g$ and mean density $\rho$ ) in a completely model-independent manner. However, the uncertainties in the results are often large. This is because, although the theory of stellar structure and evolution tell us otherwise, the equations assume that all values of $T_{\text {eff }}$ are possible for a star of a given $M$ and $R$. This freedom can often result in unphysical results. The way out is to use an approach that uses the relation between $M, R$ and $T_{\text {eff }}$ implicitly using grids of stellar models. For given values of $\Delta v, v_{\max }$ and $T_{\text {eff }}, M$ and $R$ (as well as any parameter, such as age) is obtained by searching within a grid of models. Knowing a star's metallicity helps in this.

Many grid based pipelines have been developed and used e.g., the Yale-Birmingham (YB) pipeline (Basu et al. 2012b, 2010; Gai et al. 2011), RadEx10 of Creevey et al. (2012), SEEK of Quirion et al. (2010), RADIUS of Stello et al. (2009), etc. Most of these pipelines work by finding the maximum likelihood of the set of input parameter data calculated with respect to the grid of models. For example, the YB pipeline works in the following manner: For a given observational (central) input parameter set, the first key step in the method is to generate 10000 input parameter sets by adding different random realisations of Gaussian noise to the actual (central) observational input parameter set. For each realisation, we find all models in a grid within $3 \sigma$ of a minimum uncertainty and use them to define the likelihood function as

$$
\mathcal{L}=\prod_{i=1}^{n}\left[\frac{1}{\sqrt{2 \pi} \sigma_{i}} \times \exp \left(-\chi^{2} / 2\right)\right],
$$


where

$$
\chi^{2}=\sum_{i=1}^{n}\left(\frac{q_{i}^{\mathrm{obs}}-q_{i}^{\mathrm{model}}}{\sigma^{i}}\right)^{2},
$$

where $q$ are the observations $\Delta v, v_{\max }, T_{\text {eff }}$ and $[\mathrm{Fe} / \mathrm{H}]$. The estimated property, such as radius or mass, is then the likelihood-weighted average of the properties. The 10,001 values of any given parameter, such as radius, estimated from the central value and the 10,000 realisations forms the probability distribution function that parameter. In the YB pipeline we adopt the median of the distribution as the estimated value of the parameter, and we use $1 \sigma$ limits from the median as a measure of the uncertainties. At the price of making the results slightly model dependent, the method gives us results that are physical. Characteristics and systematic biases involved with grid-based analyses have been investigated in detail by Gai et al. (2011), Basu et al. (2010), Bazot et al. (2012), and Gruberbauer et al. (2012). The systematics related to determining $\log g$ have been investigated by Creevey et al. (2013).

Grid-based modelling has been used to determine the global properties (mass, radius, density) of all the short-cadence targets observed by Kepler (Chaplin et al. 2014). This method has also being used to determine the global properties of many of the red giants observed by Kepler (Pinsonneault et al. 2014).

\subsubsection{Detailed modelling}

There are some stars for which frequencies of individual modes are available. In such cases one resorts to modelling the star, calculating the frequencies of models and comparing the frequencies of the models with that of star. The star is modelled to have the observed $T_{\text {eff }}$ and metallicity. This is, in a sense, reminiscent of what used to be done in the early days of helioseismology. The biggest difference is that unlike the Sun, we do not know the mass, radius, age and luminosity of these stars independently.

One of the biggest issues in such an analysis is the surface term. In Fig. 41, we show the échelle diagrams of the Sun and 16 Cyg A and their models. The familiar surface term is seen as the frequency offset between the two stars and their models at higher frequencies. As had been described earlier, the vexing issue of the surface term led to the development of inversion techniques. In the case of the Sun, if we wished we could easily use a subset of the modes to define the surface term and use the rest to do the analysis, though of course it is more usual to fit the surface term simultaneously. We do not have this luxury in the case of asteroseismic analysis. Thus ad hoc means are usually used to correct for the surface term, usually by scaling the solar surface term.

The most commonly use correction for the surface term is that of Kjeldsen et al. (2008). This correction is in the form of a power law derived using solar models and their frequency differences with respect to the solar frequencies. The scaling for the correction is written in the form

$$
v_{\mathrm{obs}}(n)-v_{\mathrm{ref}}(n)=a\left(\frac{v_{\mathrm{obs}}}{v_{0}}\right)^{b},
$$

where, $v_{\mathrm{obs}}$ are the observed frequencies, $v_{\text {ref }}$ are the frequencies of the model. To apply the correction one needs to specify the exponent $b$ and the reference frequency 

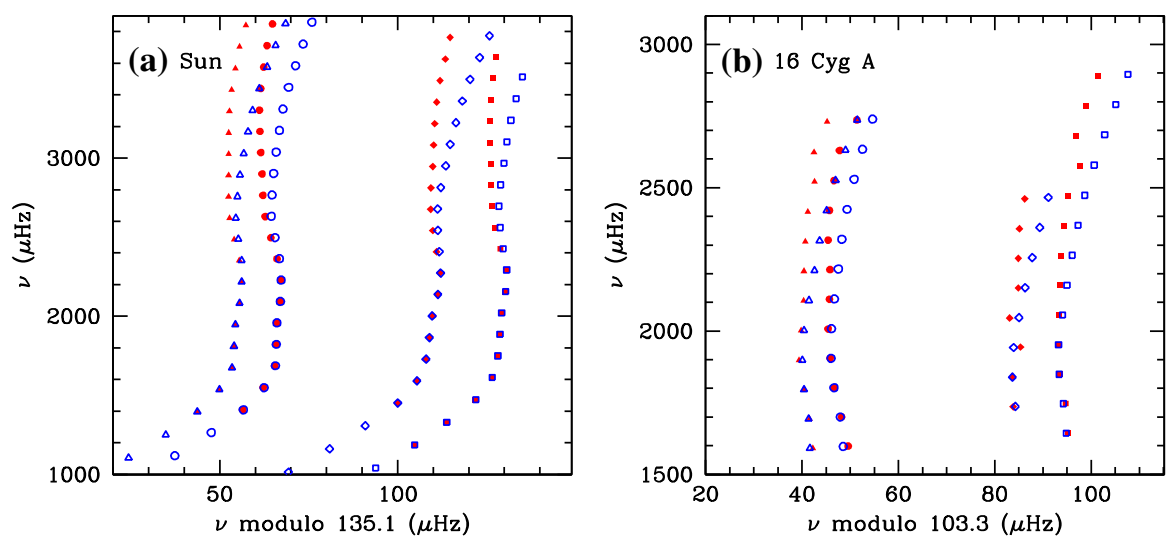

Fig. 41 a The échelle diagram for low-degree solar modes (red) and Model S (blue). b The échelle diagram for $16 \mathrm{Cyg} \mathrm{A}(\mathrm{red})$ and a model of that star (blue). Note that in both panels, the échelle diagram of the model deviates from that of the star at high frequencies. This is nothing but the surface term

$v_{0}$. The exponent is determined using the solar surface term. The quantity $a$, though formally the frequency difference at $v_{\mathrm{obs}}=v_{0}$ is often determined in an average manner. Variants of Eq. (174) are also used, e.g., Metcalfe et al. (2012) fix $v_{0}$ to be $v_{\max }$, reducing one degree of freedom. Other ways that have been used widely depend on explicitly scaling the solar surface term; Silva Aguirre et al. (2015) describe some of these methods. While the corrections are widely used, it is unlikely that the solar scaling can be used for all stars that show solar-like oscillations.

More recently, Ball and Gizon (2014), based on the work of Gough (1990), suggested two surface term corrections:

$$
\frac{c}{E}\left(\frac{v}{v_{\mathrm{ac}}}\right)^{3}
$$

and

$$
\frac{1}{E}\left[c_{3}\left(\frac{v}{v_{\mathrm{ac}}}\right)+c_{-1}\left(\frac{v}{v_{\mathrm{ac}}}\right)^{-1}\right],
$$

where, $E$ is the more inertia, and $c, c_{3}$ and $c_{-1}$ are constants obtained by fitting the frequency differences. These forms are promising and tests show that Eq. (176) works very well over a large portion of the HR diagram (Schmitt and Basu 2015) and modellers are beginning to adopt this form.

Despite the ambiguity caused by the surface term, detailed modelling gives more precise estimates of the properties of a star. Of course grid modelling is also affected by the surface term, but to a lesser degree since the term gets reduced in the process of calculating the average value of $\Delta v$. Such detailed modelling has been reported by Metcalfe et al. (2010, 2012, 2014), Deheuvels and Michel (2011), Mathur et al. (2012), Silva Aguirre et al. (2013), Silva Aguirre et al. (2015), etc. Because of the uncertainty caused by the surface term, investigators are looking into using combinations of frequencies of different modes that should be relatively insensitive to the 
surface term (e.g., Roxburgh and Vorontsov 2003, 2013; Otí Floranes et al. 2005; Roxburgh 2014).

\subsubsection{Exploiting acoustic glitches}

Asteroseismic analyses are not restricted to trying to fit the oscillation frequencies. As in the case of the Sun, investigators are using signatures of acoustic glitches to determine the positions of the helium ionisation zone and the convection-zone base.

Mazumdar et al. $(2012,2014)$ determined the acoustic depths of the He II ionisation zone of several stars. They were also successful in measuring the acoustic depths of the convection-zone bases of some of the stars. More work needs to be done to translate the acoustic depth to a physical radius that could help modellers.

Basu et al. (2004) had proposed that the amplitude of the He II acoustic glitch could be used to determine the helium abundance of stars. Verma et al. (2014) applied this idea to 16 Cyg A and 16 Cyg B using frequencies obtained from Kepler observations. These two stars are solar analogues - only slightly higher masses than the Sun and they are slightly older. The estimate of their current helium abundance is entirely consistent with the solar helium abundance with the helium abundance of $16 \mathrm{Cyg} \mathrm{A}$ in the range $0.231-0.251$ and that of 16 Cyg B in the range 0.218 and 0.266 .

\subsubsection{Analyses of internal rotation}

It is difficult to determine the rotation profile of a star using asteroseismic data. The problems lie with the data themselves $-\ell=0$ modes are not affected by rotation at all, while $\ell=1$ modes split into at most three components and $\ell=2$ modes into at most five. Whether all the splittings are visible also depend on the angle of inclination between the star and us (see e.g., Gizon and Solanki 2003). Thus data from which rotation can be determined are very limited. The visibility of the components as a function of inclination has been used successfully to study the spin-orbit misalignment in exoplanetary systems (Huber et al. 2013; Chaplin et al. 2013).

One of the first detailed investigations into the internal rotation profile of a star other than the Sun was that by Deheuvels et al. (2012). They compared the splittings of the mixed modes with those of the p modes of the star KIC 7341231 and found clear evidence of a radius-dependent rotation rate. They showed that the core of this star, which is just off the subgiant into the low end of the red-giant branch, rotates at least five times faster than the surface. The ratio between the core and surface rotation rates places constraints on models of angular momentum transfer. Deheuvels et al. (2014) looked at a sample of subgiants and found that the contrast between core and surface rotation increases as the star evolves; their results suggest that while the cores spin up, the envelope spins down. Data for at least two stars suggest a sharp discontinuity in rotation between the core and the envelope located at the hydrogen burning shell. The implications of these results are still being studied.

Beck et al. (2012) and Mosser et al. (2012) have concentrated on determining and interpreting the rotational splittings of red giants. Mode splittings in red giants are dominated by the rotation rate of the core. The data suggest that the core rotates faster 
as it ascends the giant branch and it appears that there is a slow-down in red-clump stars compared with stars on the ascending part of the giant branch.

The most Sun-like stars that have been studied are the two components of the wide binary system 16 Cyg. Davies et al. (2015) found that the data only allow the estimation of an average rotation rate. $16 \mathrm{Cyg} \mathrm{A}$ was found to have a rotation period of $23.8_{-1.8}^{+1.5}$ days, while the rotation rate of $16 \mathrm{Cyg} \mathrm{B}$ is $23.2_{-3.2}^{+11.5}$ days.

The study of other Sun-like stars have not yet yielded any notable results. There have however, been several investigations into how one could study the internal rotation of these stars. Studies include that of Lund et al. (2014) who studied the impact of different differential rotation profiles on the splittings of p-mode oscillation frequencies, and Schunker et al. (2016a) who examined the sensitivity of asteroseismic rotation inversions to uncertainties in the data. Schunker et al. (2016b) also examined whether one could find evidence of radial differential rotation in an average-sense by inverting data for an ensemble of stars. This has not yet been applied to real data yet.

Gizon and Solanki (2004) had shown how one might be able to determine differential rotation of a star, i.e., variation of rotation with latitude, using seismic data, however results have been elusive. The data are not sensitive enough. This is not completely surprising according to Ballot et al. (2006a), but disappointing nevertheless.

A summary of recent developments in this field can be found in Aerts (2015).

\section{Concluding thoughts}

Helioseismology has revolutionised our knowledge of the Sun. It has allowed us to know the structure and dynamics of the solar interior very well. However, what is clearly missing is better knowledge of the near-surface layers of the Sun. Accurate and precise frequencies of high-degree will resolve this issue. Additionally, a concerted push to observe low-frequency low-degree $\mathrm{p}$ modes and g modes will help clarify some of the issues that remain about the structure and dynamics of the solar core.

Our database of helioseismic observations does not go very far back in time. There have been continuous observations of low-degree modes for the last 30 years, and of intermediate degree modes for only 20 years. These observations have given us tantalising glimpses of how the Sun changes over a solar cycle, and on how one cycle can be very different from another. A 20-year database is definitely not long enough to learn what the interior of the Sun behaves like in a "typical" cycle, assuming of course, that a typical cycle can be defined. We thus need to continue these observations as long as we can.

Asteroseismology is in an ascending phase. These are exciting times with newer and better data being available each day. However, in the rush to exploit these data, we seem to have forgotten that we need really good analysis techniques to make optimum use of the data - a case in point is the treatment of the surface term. Now that the data stream from Kepler has slowed down, and TESS and PLATO are still a few years off, this is perhaps the time to figure out newer and better techniques of analysing these data. 
Acknowledgements I would like to thank the two anonymous referees who have helped improve this review. I would also like to thank Charles Baldner, Anne-Marie Broomhall, William Chaplin, Rachel Howe and Kiran Jain for preparing some of the figures that appear in this review, and thanks are due to Tim Larson for early access to reprocessed MDI data.

Open Access This article is distributed under the terms of the Creative Commons Attribution 4.0 International License (http://creativecommons.org/licenses/by/4.0/), which permits unrestricted use, distribution, and reproduction in any medium, provided you give appropriate credit to the original author(s) and the source, provide a link to the Creative Commons license, and indicate if changes were made.

\section{References}

Abdurashitov JN, Faizov EL, Gavrin VN, Gusev AO, Kalikhov AV, Knodel TV, Knyshenko II, Kornoukhov VN, Mirmov IN, Pshukov AM, Shalagin AM, Shikhin AA, Timofeyev PV, Veretenkin EP, Vermul VM, Zatsepin GT, Bowles TJ, Nico JS, Teasdale WA, Wark DL, Wilkerson JF, Cleveland BT, Daily T, Davis R, Lande K, Lee CK, Wildenhain PW, Elliott SR, Cherry ML, Kouzes RT (1994) Results from SAGE (the Russian-American gallium solar neutrino experiment). Phys Lett B 328:234-248. doi:10. 1016/0370-2693(94)90454-5

Adelberger EG, Austin SM, Bahcall JN, Balantekin AB, Bogaert G, Brown LS, Buchmann L, Cecil FE, Champagne AE, de Braeckeleer L, Duba CA, Elliott SR, Freedman SJ, Gai M, Goldring G, Gould CR, Gruzinov A, Haxton WC, Heeger KM, Henley E, Johnson CW, Kamionkowski M, Kavanagh RW, Koonin SE, Kubodera K, Langanke K, Motobayashi T, Pandharipande V, Parker P, Robertson RG, Rolfs C, Sawyer RF, Shaviv N, Shoppa TD, Snover KA, Swanson E, Tribble RE, Turck-Chièze S, Wilkerson JF (1998) Solar fusion cross sections. Rev Mod Phys 70:1265-1291. doi:10.1103/RevModPhys.70. 1265. arXiv:astro-ph/9805121

Adelberger EG, García A, Robertson RGH, Snover KA, Balantekin AB, Heeger K, Ramsey-Musolf MJ, Bemmerer D, Junghans A, Bertulani CA, Chen JW, Costantini H, Prati P, Couder M, Uberseder E, Wiescher M, Cyburt R, Davids B, Freedman SJ, Gai M, Gazit D, Gialanella L, Imbriani G, Greife U, Hass M, Haxton WC, Itahashi T, Kubodera K, Langanke K, Leitner D, Leitner M, Vetter P, Winslow L, Marcucci LE, Motobayashi T, Mukhamedzhanov A, Tribble RE, Nollett KM, Nunes FM, Park TS, Parker PD, Schiavilla R, Simpson EC, Spitaleri C, Strieder F, Trautvetter HP, Suemmerer K, Typel S (2011) Solar fusion cross sections. II. The $p p$ chain and CNO cycles. Rev Mod Phys 83:195-246. doi:10.1103/RevModPhys.83.195. arXiv:1004.2318

Aerts C (2015) The age and interior rotation of stars from asteroseismology. Astron Nachr 336:477. doi:10. 1002/asna.201512177. arXiv:1503.06690

Ahmad QR, Allen RC, Andersen TC, Anglin JD, Barton JC, Beier EW, Bercovitch M, Bigu J, Biller SD, Black RA, Blevis I, Boardman RJ, Boger J, Bonvin E, Boulay MG, Bowler MG, Bowles TJ, Brice SJ, Browne MC, Bullard TV, Bühler G, Cameron J, Chan YD, Chen HH, Chen M, Chen X, Cleveland BT, Clifford ET, Cowan JH, Cowen DF, Cox GA, Dai X, Dalnoki-Veress F, Davidson WF, Doe PJ, Doucas G, Dragowsky MR, Duba CA, Duncan FA, Dunford M, Dunmore JA, Earle ED, Elliott SR, Evans HC, Ewan GT, Farine J, Fergani H, Ferraris AP, Ford RJ, Formaggio JA, Fowler MM, Frame K, Frank ED, Frati W, Gagnon N, Germani JV, Gil S, Graham K, Grant DR, Hahn RL, Hallin AL, Hallman ED, Hamer AS, Hamian AA, Handler WB, Haq RU, Hargrove CK, Harvey PJ, Hazama R, Heeger KM, Heintzelman WJ, Heise J, Helmer RL, Hepburn JD, Heron H, Hewett J, Hime A, Howe M, Hykawy JG, Isaac MC, Jagam P, Jelley NA, Jillings C, Jonkmans G, Kazkaz K, Keener PT, Klein JR, Knox AB, Komar RJ, Kouzes R, Kutter T, Kyba CC, Law J, Lawson IT, Lay M, Lee HW, Lesko KT, Leslie JR, Levine I, Locke W, Luoma S, Lyon J, Majerus S, Mak HB, Maneira J, Manor J, Marino AD, McCauley N, McDonald AB, McDonald DS, McFarlane K, McGregor G, Meijer Drees R, Mifflin C, Miller GG, Milton G, Moffat BA, Moorhead M, Nally CW, Neubauer MS, Newcomer FM, Ng HS, Noble AJ, Norman EB, Novikov VM, O’Neill M, Okada CE, Ollerhead RW, Omori M, Orrell JL, Oser SM, Poon AW, Radcliffe TJ, Roberge A, Robertson BC, Robertson RG, Rosendahl SS, Rowley JK, Rusu VL, Saettler E, Schaffer KK, Schwendener MH, Schülke A, Seifert H, Shatkay M, Simpson JJ, Sims CJ, Sinclair D, Skensved P, Smith AR, Smith MW, Spreitzer T, Starinsky N, Steiger TD, Stokstad RG, Stonehill LC, Storey RS, Sur B, Tafirout R, Tagg N, Tanner NW, Taplin RK, Thorman M, Thornewell PM, Trent PT, Tserkovnyak YI, van Berg R, van de Water RG, Virtue CJ, Waltham CE, Wang JX, Wark DL, West N, Wilhelmy JB, Wilkerson JF, Wilson JR, Wittich P, Wouters JM, 
Yeh M (2002) Direct evidence for neutrino flavor transformation from neutral-current interactions in the sudbury neutrino observatory. Phys Rev Lett 89:011301. doi:10.1103/PhysRevLett.89.011301. arXiv:nucl-ex/0204008

Allen CW (1973) Astrophysical quantities, 3rd edn. Athlone Press, London

Allende Prieto C, Lambert DL, Asplund M (2001) The forbidden abundance of oxygen in the Sun. Astrophys J Lett 556:L63-L66. doi:10.1086/322874. arXiv:astro-ph/0106360

Allende Prieto C, Lambert DL, Asplund M (2002) A reappraisal of the solar photospheric C/O ratio. Astrophys J Lett 573:L137-L140. doi:10.1086/342095. arXiv:astro-ph/0206089

Anders E, Grevesse N (1989) Abundances of the elements: meteoritic and solar. Geochim Cosmochim Acta 53:197-214. doi:10.1016/0016-7037(89)90286-X

Ando H, Osaki Y (1975) Nonadiabatic nonradial oscillations: an application to the five-minute oscillation of the sun. Publ Astron Soc Japan 27:581-603

Anguera Gubau M, Pallé PL, Pérez Hernández F, Régulo C, Roca Cortés T (1992) The low $l$ solar p-mode spectrum at maximum and minimum solar activity. Astron Astrophys 255:363-372

Angulo C, Arnould M, Rayet M, Descouvemont P, Baye D, Leclercq-Willain C, Coc A, Barhoumi S, Aguer P, Rolfs C, Kunz R, Hammer JW, Mayer A, Paradellis T, Kossionides S, Chronidou C, Spyrou K, Degl'Innocenti S, Fiorentini G, Ricci B, Zavatarelli S, Providencia C, Wolters H, Soares J, Grama C, Rahighi J, Shotter A, Lamehi Rachti M (1999) A compilation of charged-particle induced thermonuclear reaction rates. Nucl Phys A 656:3-183. doi:10.1016/S0375-9474(99)00030-5

Anselmann P, Hampel W, Heusser G, Kiko J, Kirsten T, Laubenstein M, Pernicka E, Pezzoni S, Rönn U, Sann M, Schlosser C, Wink R, Wójcik M, von Ammon R, Ebert KH, Fritsch T, Heidt D, Henrich E, Stieglitz L, Weirich F, Balata M, Lalla H, Bellotti E, Cattadori C, Cremonesi O, Ferrari N, Fiorini E, Zanotti L, Altmann M, von Feilitzsch F, Mößbauer R, Schanda U, Berthomieu G, Schatzman E, Carmi I, Dostrovsky I, Bacci C, Belli P, Bernabei R, D’Angelo S, Paoluzi L, Bevilacqua A, Charbit S, Cribier M, Gosset L, Rich J, Spiro M, Stolarczyk T, Tao C, Vignaud D, Boger J, Hahn RL, Hartmann FX, Rowley JK, Stoenner RW, Weneser J (1995) GALLEX solar neutrino observations: complete results for GALLEX II. Phys Lett B 357:237-247. doi:10.1016/0370-2693(95)00897-T

Antia HM (1995) Effects of surface layers on helioseismic inversion. Mon Not R Astron Soc 274:499-503. doi:10.1093/mnras/274.2.499

Antia HM (1996) Nonasymptotic helioseismic inversion: iterated seismic solar model. Astron Astrophys 307:609-623

Antia HM (1998) Estimate of solar radius from f-mode frequencies. Astron Astrophys 330:336-340. arXiv:astro-ph/9707226

Antia HM (2002) Subsurface magnetic fields from helioseismology. In: Sawaya-Lacoste H (ed) SOLMAG 2002, ESA Special Publication, vol 505. ESA, Nordwijk, pp 71-78. arXiv:astro-ph/0208339

Antia HM, Basu S (1994a) Measuring the helium abundance in the solar envelope: the role of the equation of state. Astrophys J 426:801-811. doi:10.1086/174117

Antia HM, Basu S (1994b) Nonasymptotic helioseismic inversion for solar structure. Astron Astrophys Suppl Ser 107:421-444

Antia HM, Basu S (2000) Temporal variations of the rotation rate in the solar interior. Astrophys J 541:442448. doi:10.1086/309421. arXiv:astro-ph/0004335

Antia HM, Basu S (2001) Temporal variations of the solar rotation rate at high latitudes. Astrophys J Lett 559:L67-L70. doi:10.1086/323701. arXiv:astro-ph/0108226

Antia HM, Basu S (2006) Determining solar abundances using helioseismology. Astrophys J 644:12921298. doi:10.1086/503707. arXiv:astro-ph/0603001

Antia HM, Basu S (2010) Solar rotation rate during the cycle 24 minimum in activity. Astrophys J 720:494502. doi:10.1088/0004-637X/720/1/494. arXiv:1008.1787

Antia HM, Basu S (2011) Revisiting the solar tachocline: average properties and temporal variations. Astrophys J Lett 735:L45. doi:10.1088/2041-8205/735/2/L45. arXiv:1107.1004

Antia HM, Basu S (2013) Contrasting the solar rotation rate of cycles 23 and 24. J Phys Conf Ser 440:012018. doi:10.1088/1742-6596/440/1/012018

Antia HM, Chitre SM (1997) Helioseismic models and solar neutrino fluxes. Mon Not R Astron Soc 289:L1-L4. doi:10.1093/mnras/289.2.L1

Antia HM, Chitre SM (1998) Determination of temperature and chemical composition profiles in the solar interior from seismic models. Astron Astrophys 339:239-251. arXiv:astro-ph/9710159

Antia HM, Chitre SM (1999) Limits on the proton-proton reaction cross-section from helioseismology. Astron Astrophys 347:1000-1004. arXiv:astro-ph/9906007 
Antia HM, Chitre SM (2002) Helioseismic limit on heavy element abundance. Astron Astrophys 393:L95L98. doi:10.1051/0004-6361:20021253. arXiv:astro-ph/0209134

Antia HM, Chitre SM, Narasimha D (1982) Overstability of acoustic modes and the solar five-minute oscillations. Solar Phys 77:303-327. doi:10.1007/BF00156114

Antia HM, Chitre SM, Gough DO (1988) On the excitation of solar 5-minute oscillations. In: ChristensenDalsgaard J, Frandsen S (eds) Advances in helio- and asteroseismology, IAU Symposium, vol 123. Aarhus University Press, Aarhus, p 371

Antia HM, Basu S, Pintar J, Pohl B (2000a) Solar cycle variation in solar f-mode frequencies and radius. Solar Phys 192:459-468. doi:10.1023/A:1005204800696. arXiv:astro-ph/0001293

Antia HM, Chitre SM, Thompson MJ (2000b) The Sun's acoustic asphericity and magnetic fields in the solar convection zone. Astron Astrophys 360:335-344. arXiv:astro-ph/0005587

Antia HM, Basu S, Hill F, Howe R, Komm RW, Schou J (2001) Solar-cycle variation of the sound-speed asphericity from GONG and MDI data 1995-2000. Mon Not R Astron Soc 327:1029-1040. doi:10. 1046/j.1365-8711.2001.04819.x. arXiv:astro-ph/0109326

Antia HM, Basu S, Pintar J, Schou J (2001b) How correlated are f-mode frequencies with solar activity? In: Wilson A, Pallé PL (eds) SOHO 10/GONG 2000 workshop: helio- and asteroseismology at the dawn of the millennium, ESA Special Publication, vol 464. ESA, Noordwijk, pp 27-32

Antia HM, Basu S, Chitre SM (2008) Solar rotation rate and its gradients during cycle 23. Astrophys J 681:680-692. doi:10.1086/588523. arXiv:0803.3619

Appourchaux T, Pallé PL (2013) The history of the g-mode quest. In: Jain K, Tripathy SC, Hill F, Leibacher JW, Pevtsov AA (eds) Fifty years of seismology of the sun and stars, ASP Conference Proceedings, vol 478. Astronomical Society of the Pacific, San Francisco, p 125

Appourchaux T, Belkacem K, Broomhall AM, Chaplin WJ, Gough DO, Houdek G, Provost J, Baudin F, Boumier P, Elsworth Y, García RA, Andersen BN, Finsterle W, Fröhlich C, Gabriel A, Grec G, Jiménez A, Kosovichev A, Sekii T, Toutain T, Turck-Chièze S (2010) The quest for the solar g modes. Astron Astrophys Rev 18:197-277. doi:10.1007/s00159-009-0027-z. arXiv:0910.0848

Arentoft T, Kjeldsen H, Bedding TR, Bazot M, Christensen-Dalsgaard J, Dall TH, Karoff C, Carrier F, Eggenberger P, Sosnowska D, Wittenmyer RA, Endl M, Metcalfe TS, Hekker S, Reffert S, Butler RP, Bruntt H, Kiss LL, O'Toole SJ, Kambe E, Ando H, Izumiura H, Sato B, Hartmann M, Hatzes A, Bouchy F, Mosser B, Appourchaux T, Barban C, Berthomieu G, Garcia RA, Michel E, Provost J, Turck-Chièze S, Martić M, Lebrun JC, Schmitt J, Bertaux JL, Bonanno A, Benatti S, Claudi RU, Cosentino R, Leccia S, Frandsen S, Brogaard K, Glowienka L, Grundahl F, Stempels E (2008) A multisite campaign to measure solar-like oscillations in procyon. I. Observations, data reduction, and slow variations. Astrophys J 687:1180-1190. doi:10.1086/592040. arXiv:0807.3794

Arnett D, Meakin C, Young PA (2010) Convection theory and sub-photospheric stratification. Astrophys J 710:1619-1626. doi:10.1088/0004-637X/710/2/1619. arXiv:0910.0821

Asplund M (2000) Line formation in solar granulation. III. The photospheric Si and meteoritic Fe abundances. Astron Astrophys 359:755-758. arXiv:astro-ph/0005322

Asplund M, Grevesse N, Sauval AJ, Allende Prieto C, Kiselman D (2004) Line formation in solar granulation. IV. [O I], and $\mathrm{OH}$ lines and the photospheric O abundance. Astron Astrophys 417:751-768. doi:10.1051/0004-6361:20034328. arXiv:astro-ph/0312290

Asplund M, Grevesse N, Sauval AJ (2005a) The solar chemical composition. In: Barnes TG III, Bash FN (eds) Cosmic abundances as records of stellar evolution and nucleosynthesis, ASP Conference Series, vol 336. Astronomical Society of the Pacific, San Francisco, p 25

Asplund M, Grevesse N, Sauval AJ, Allende Prieto C, Blomme R (2005b) Line formation in solar granulation. VI. [C I], $\mathrm{CH}$ and $\mathrm{C}_{2}$ lines and the photospheric $\mathrm{C}$ abundance. Astron Astrophys 431:693-705. doi:10.1051/0004-6361:20041951. arXiv:astro-ph/0410681

Asplund M, Grevesse N, Sauval AJ, Allende Prieto C, Kiselman D (2005c) Line formation in solar granulation. IV. [O I], and $\mathrm{OH}$ lines and the photospheric O abundance. Astron Astrophys 435:339-340. doi:10.1051/0004-6361:20034328e

Asplund M, Grevesse N, Sauval AJ, Scott P (2009) The chemical composition of the Sun. Annu Rev Astron Astrophys 47:481-522. doi:10.1146/annurev.astro.46.060407.145222. arXiv:0909.0948

Auvergne M, Bodin P, Boisnard L, Buey JT, Chaintreuil S, Epstein G, Jouret M, Lam-Trong T, Levacher P, Magnan A, Perez R, Plasson P, Plesseria J, Peter G, Steller M, Tiphène D, Baglin A, Agogué P, Appourchaux T, Barbet D, Beaufort T, Bellenger R, Berlin R, Bernardi P, Blouin D, Boumier P, Bonneau F, Briet R, Butler B, Cautain R, Chiavassa F, Costes V, Cuvilho J, Cunha-Parro V, de Oliveira FF, Decaudin M, Defise JM, Djalal S, Docclo A, Drummond R, Dupuis O, Exil G, Fauré C, Gaboriaud 
A, Gamet P, Gavalda P, Grolleau E, Gueguen L, Guivarc'h V, Guterman P, Hasiba J, Huntzinger G, Hustaix H, Imbert C, Jeanville G, Johlander B, Jorda L, Journoud P, Karioty F, Kerjean L, Lafond L, Lapeyrere V, Landiech P, Larqué T, Laudet P, Le Merrer J, Leporati L, Leruyet B, Levieuge B, Llebaria A, Martin L, Mazy E, Mesnager JM, Michel JP, Moalic JP, Monjoin W, Naudet D, Neukirchner S, Nguyen-Kim K, Ollivier M, Orcesi JL, Ottacher H, Oulali A, Parisot J, Perruchot S, Piacentino A, Pinheiro da Silva L, Platzer J, Pontet B, Pradines A, Quentin C, Rohbeck U, Rolland G, Rollenhagen F, Romagnan R, Russ N, Samadi R, Schmidt R, Schwartz N, Sebbag I, Smit H, Sunter W, Tello M, Toulouse P, Ulmer B, Vandermarcq O, Vergnault E, Wallner R, Waultier G, Zanatta P (2009) The CoRoT satellite in flight: description and performance. Astron Astrophys 506:411-424. doi:10.1051/ 0004-6361/200810860. arXiv:0901.2206

Bachmann KT, Brown TM (1993) P-mode frequency variation in relation to global solar activity. Astrophys J Lett 411:L45-L48. doi:10.1086/186908

Backus G, Gilbert F (1970) Uniqueness in the inversion of inaccurate gross earth data. Philos Trans R Soc Lond Ser A 266:123-192. doi:10.1098/rsta.1970.0005

Backus GE, Gilbert JF (1968) The resolving power of gross earth data. Geophys J 16:169-205. doi:10. 1111/j.1365-246X.1968.tb00216.x

Badnell NR, Bautista MA, Butler K, Delahaye F, Mendoza C, Palmeri P, Zeippen CJ, Seaton MJ (2005) Updated opacities from the Opacity Project. Mon Not R Astron Soc 360:458-464. doi:10.1111/j. 1365-2966.2005.08991.x. arXiv:astro-ph/0410744

Baglin A, Auvergne M, Barge P, Deleuil M, Catala C, Michel E, Weiss W, Team C (2006) Scientific objectives for a minisat: CoRoT. In: Fridlund M, Baglin A, Lochard J, Conroy L (eds) The CoRoT mission pre-launch status: stellar seismology and planet finding, ESA Special Publication, vol SP1306. ESA, Nordwijk, p 33

Bahcall JN (2003) Solar models: an historical overview. In: Shrock R (ed) Neutrinos and implications for physics beyond the standard model. World Scientific, Singapore, p 15. doi:10.1142/ 9789812704207_0004

Bahcall JN, Pinsonneault MH (1992) Standard solar models, with and without helium diffusion, and the solar neutrino problem. Rev Mod Phys 64:885-926. doi:10.1103/RevModPhys.64.885

Bahcall JN, Sears RL (1972) Solar neutrinos. Annu Rev Astron Astrophys 10:25. doi:10.1146/annurev.aa. 10.090172 .000325

Bahcall JN, Serenelli AM (2005) How do uncertainties in the surface chemical composition of the Sun affect the predicted solar neutrino fluxes? Astrophys J 626:530-542. doi:10.1086/429883. arXiv:astro-ph/0412096

Bahcall JN, Shaviv G (1968) Solar models and neutrino fluxes. Astrophys J 153:113. doi:10.1086/149641

Bahcall JN, Ulrich RK (1988) Solar models, neutrino experiments, and helioseismology. Rev Mod Phys 60:297-372. doi:10.1103/RevModPhys.60.297

Bahcall JN, Fowler WA, Iben I Jr, Sears RL (1963) Solar neutrino flux. Astrophys J 137:344-346. doi:10. $1086 / 147513$

Bahcall JN, Bahcall NA, Ulrich RK (1968) Mixing in the Sun and neutrino fluxes. Astrophys Lett Commun 2:91. doi:10.1086/149641

Bahcall JN, Huebner WF, Lubow SH, Parker PD, Ulrich RK (1982) Standard solar models and the uncertainties in predicted capture rates of solar neutrinos. Rev Mod Phys 54:767-799. doi:10.1103/ RevModPhys.54.767

Bahcall JN, Pinsonneault MH, Wasserburg GJ (1995) Solar models with helium and heavy-element diffusion. Rev Mod Phys 67:781-808. doi:10.1103/RevModPhys.67.781. arXiv:hep-ph/9505425

Bahcall JN, Pinsonneault MH, Basu S, Christensen-Dalsgaard J (1997) Are standard solar models reliable? Phys Rev Lett 78:171-174. doi:10.1103/PhysRevLett.78.171. arXiv:astro-ph/9610250

Bahcall JN, Basu S, Pinsonneault MH (1998) How uncertain are solar neutrino predictions? Phys Lett B 433:1-8. doi:10.1016/S0370-2693(98)00657-1. arXiv:astro-ph/9805135

Bahcall JN, Basu S, Pinsonneault M, Serenelli AM (2005a) Helioseismological implications of recent solar abundance determinations. Astrophys J 618:1049-1056. doi:10.1086/426070. arXiv:astro-ph/0407060

Bahcall JN, Serenelli AM, Basu S (2005b) New solar opacities, abundances, helioseismology, and neutrino fluxes. Astrophys J Lett 621:L85-L88. doi:10.1086/428929. arXiv:astro-ph/0412440

Bahcall JN, Serenelli AM, Basu S (2006) 10,000 standard solar models: a Monte Carlo simulation. Astrophys J Suppl Ser 165:400-431. doi:10.1086/504043. arXiv:astro-ph/0511337 
Baldner CS, Basu S (2008) Solar cycle related changes at the base of the convection zone. Astrophys J 686:1349-1361. doi:10.1086/591514. arXiv:0807.0442

Baldner CS, Antia HM, Basu S, Larson TP (2009) Solar magnetic field signatures in helioseismic splitting coefficients. Astrophys J 705:1704-1713. doi:10.1088/0004-637X/705/2/1704. arXiv:0910.1597

Ball WH, Gizon L (2014) A new correction of stellar oscillation frequencies for near-surface effects. Astron Astrophys 568:A123. doi:10.1051/0004-6361/201424325. arXiv:1408.0986

Ballot J, García RA, Lambert P (2006) Rotation speed and stellar axis inclination from p modes: how CoRoT would see other suns. Mon Not R Astron Soc 369:1281-1286. doi:10.1111/j.1365-2966.2006.10375. X. arXiv:astro-ph/0603671

Ballot J, Jiménez-Reyes SJ, García RA (2006) How GOLF sees the second helium ionization layer across the solar cycle. In: Thompson M (ed) SOHO 18/Gong 2006/HELAS I: beyond the spherical Sun, ESA Special Publication, vol SP-624. ESA, Noordwijk

Ballot J, Gizon L, Samadi R, Vauclair G, Benomar O, Bruntt H, Mosser B, Stahn T, Verner GA, Campante TL, García RA, Mathur S, Salabert D, Gaulme P, Régulo C, Roxburgh IW, Appourchaux T, Baudin F, Catala C, Chaplin WJ, Deheuvels S, Michel E, Bazot M, Creevey O, Dolez N, Elsworth Y, Sato KH, Vauclair S, Auvergne M, Baglin A (2011) Accurate p-mode measurements of the G0V metal-rich CoRoT target HD 52265. Astron Astrophys 530:A97. doi:10.1051/0004-6361/201116547. arXiv:1105.3551

Balmforth NJ (1992) Solar pulsational stability: part three-acoustical excitation by turbulent convection. Mon Not R Astron Soc 255:639. doi:10.1093/mnras/255.4.639

Balmforth NJ, Gough DO (1990) Effluent stellar pulsation. Astrophys J 362:256-266. doi:10.1086/169262

Balmforth NJ, Gough DO, Merryfield WJ (1996) Structural changes to the Sun through the solar cycle. Mon Not R Astron Soc 278:437-448. doi:10.1093/mnras/278.2.437

Basu S (1997) Seismology of the base of the solar convection zone. Mon Not R Astron Soc 288:572-584

Basu S (1998) Effects of errors in the solar radius on helioseismic inferences. Mon Not R Astron Soc 298:719-728. doi:10.1046/j.1365-8711.1998.01690.x. arXiv:astro-ph/9712133

Basu S (2002) What does helioseismology tell us about solar cycle related structural changes in the Sun? In: Wilson A (ed) From solar min to max: half a solar cycle with SOHO, ESA Special Publication, vol SP-508. ESA, Noordwijk, pp 7-14. arXiv:astro-ph/0205049

Basu S (2013) The peculiar solar cycle 24-where do we stand? J Phys Conf Ser 440:012001. doi:10.1088/ $1742-6596 / 440 / 1 / 012001$

Basu S (2014) Studying stars through frequency inversions. In: Pallé P, Esteban C (eds) Asteroseismology, vol 22. Cambridge University Press, Cambridge; Canary Islands Winter School of Astrophysics, New York, pp 87-122

Basu S, Antia HM (1994) Seismology of the solar convection zone. J Astrophys Astron 15:143-156. doi:10. 1007/BF03012174

Basu S, Antia HM (1995) Helium abundance in the solar envelope. Mon Not R Astron Soc 276:1402-1408

Basu S, Antia HM (1997) Seismic measurement of the depth of the solar convection zone. Mon Not R Astron Soc 287:189-198

Basu S, Antia HM (2000) Possible solar cycle variations in the convection zone. Solar Phys 192:449-458. doi:10.1023/A:1005232901528. arXiv:astro-ph/0001444

Basu S, Antia HM (2001) A study of possible temporal and latitudinal variations in the properties of the solar tachocline. Mon Not R Astron Soc 324:498-508. doi:10.1046/j.1365-8711.2001.04364.x. arXiv:astro-ph/0101314

Basu S, Antia HM (2001b) A study of temporal variations of the tachocline. In: Wilson A, Pallé PL (eds) Helio- and asteroseismology at the dawn of the millennium, ESA Special Publication, vol 464. ESA, Nordwijk, pp 297-300

Basu S, Antia HM (2003) Changes in solar dynamics from 1995 to 2002. Astrophys J 585:553-565. doi:10. 1086/346020. arXiv:astro-ph/0211548

Basu S, Antia HM (2004) Constraining solar abundances using helioseismology. Astrophys J Lett 606:L85L88. doi:10.1086/421110. arXiv:astro-ph/0403485

Basu S, Antia HM (2008) Helioseismology and solar abundances. Phys Rep 457:217-283. doi:10.1016/j. physrep.2007.12.002. arXiv:0711.4590

Basu S, Antia HM (2013) Revisiting the issue of solar abundances. J Phys Conf Ser 440:012017. doi:10. $1088 / 1742-6596 / 440 / 1 / 012017$

Basu S, Christensen-Dalsgaard J (1997) Equation of state and helioseismic inversions. Astron Astrophys 322:L5-L8. arXiv:astro-ph/9702162 
Basu S, Mandel A (2004) Does solar structure vary with solar magnetic activity? Astrophys J Lett 617:L155L158. doi:10.1086/427435. arXiv:astro-ph/0411427

Basu S, Thompson MJ (1996) On constructing seismic models of the Sun. Astron Astrophys 305:631

Basu S, Antia HM, Narasimha D (1994) Helioseismic measurement of the extent of overshoot below the solar convection zone. Mon Not R Astron Soc 267:209

Basu S, Christensen-Dalsgaard J, Pérez Hernández F, Thompson MJ (1996a) Filtering out near-surface uncertainties from helioseismic inversions. Mon Not R Astron Soc 280:651

Basu S, Christensen-Dalsgaard J, Schou J, Thompson MJ, Tomczyk S (1996b) The Sun's hydrostatic structure from LOWL data. Astrophys J 460:1064. doi:10.1086/177032

Basu S, Christensen-Dalsgaard J, Chaplin WJ, Elsworth Y, Isaak GR, New R, Schou J, Thompson MJ, Tomczyk S (1997) Solar internal sound speed as inferred from combined BiSON and LOWL oscillation frequencies. Mon Not R Astron Soc 292:243. doi:10.1093/mnras/292.2.243. arXiv:astro-ph/9702105

Basu S, Däppen W, Nayfonov A (1999) Helioseismic analysis of the hydrogen partition function in the solar interior. Astrophys J 518:985-993. doi:10.1086/307312. arXiv:astro-ph/9810132

Basu S, Pinsonneault MH, Bahcall JN (2000a) How much do helioseismological inferences depend on the assumed reference model? Astrophys J 529:1084-1100. doi:10.1086/308302. arXiv:astro-ph/9909247

Basu S, Turck-Chièze S, Berthomieu G, Brun AS, Corbard T, Gonczi G, Christensen-Dalsgaard J, Provost J, Thiery S, Gabriel AH, Boumier P (2000b) Structure of the solar core: effect of asymmetry of peak profiles. Astrophys J 535:1078-1084. doi:10.1086/308875. arXiv:astro-ph/0001208

Basu S, Mazumdar A, Antia HM, Demarque P (2004) Asteroseismic determination of helium abundance in stellar envelopes. Mon Not R Astron Soc 350:277-286. doi:10.1111/j.1365-2966.2004.07644.x. arXiv:astro-ph/0402360

Basu S, Chaplin WJ, Elsworth Y, New R, Serenelli AM (2009) Fresh insights on the structure of the solar core. Astrophys J 699:1403-1417. doi:10.1088/0004-637X/699/2/1403. arXiv:0905.0651

Basu S, Chaplin WJ, Elsworth Y (2010) Determination of stellar radii from asteroseismic data. Astrophys J 710:1596-1609. doi:10.1088/0004-637X/710/2/1596. arXiv:0909.0506

Basu S, Broomhall AM, Chaplin WJ, Elsworth Y (2012a) Thinning of the Sun's magnetic layer: the peculiar solar minimum could have been predicted. Astrophys J 758:43. doi:10.1088/0004-637X/758/1/43. arXiv: 1208.5493

Basu S, Verner GA, Chaplin WJ, Elsworth Y (2012b) Effect of uncertainties in stellar model parameters on estimated masses and radii of single stars. Astrophys J 746:76. doi:10.1088/0004-637X/746/1/76. arXiv:1111.6976

Basu S, Grevesse N, Mathis S, Turck-Chièze S (2015) Understanding the internal chemical composition and physical processes of the solar interior. Space Sci Rev 196:49-77. doi:10.1007/s11214-014-0035-9

Bazot M, Bourguignon S, Christensen-Dalsgaard J (2012) A Bayesian approach to the modelling of $\alpha$ Cen A. Mon Not R Astron Soc 427:1847-1866. doi:10.1111/j.1365-2966.2012.21818.x. arXiv:1209.0222

Beck JG (2000) A comparison of differential rotation measurements. Solar Phys 191:47-70. doi:10.1023/ A: 1005226402796

Beck PG, De Ridder J, Aerts C, Kallinger T, Hekker S, García RA, Mosser B, Davies GR (2012) Constraining the core-rotation rate in red-giant stars from Kepler space photometry. Astron Nachr 333:967. doi:10. 1002/asna.201211787. arXiv:1211.0153

Bedding TR (2014) Solar-like oscillations: an observational perspective. In: Pallé PL, Esteban C (eds) Asteroseismology. Cambridge University Press, Cambridge, p 60

Bedding TR, Kjeldsen H (2003) Solar-like oscillations. Pub Astron Soc Aust 20:203-212. doi:10.1071/ AS03025. arXiv:astro-ph/0305425

Bedding TR, Kjeldsen H, Butler RP, McCarthy C, Marcy GW, O’Toole SJ, Tinney CG, Wright JT (2004) Oscillation frequencies and mode lifetimes in $\alpha$ centauri A. Astrophys J 614:380-385. doi:10.1086/ 423484. arXiv:astro-ph/0406471

Bedding TR, Kjeldsen H, Arentoft T, Bouchy F, Brandbyge J, Brewer BJ, Butler RP, Christensen-Dalsgaard J, Dall T, Frandsen S, Karoff C, Kiss LL, Monteiro MJPFG, Pijpers FP, Teixeira TC, Tinney CG, Baldry IK, Carrier F, O'Toole SJ (2007) Solar-like oscillations in the G2 subgiant $\beta$ hydri from dual-site observations. Astrophys J 663:1315-1324. doi:10.1086/518593. arXiv:astro-ph/0703747

Belkacem K, Samadi R (2013) Connections between stellar oscillations and turbulent convection. In: Goupil M, Belkacem K, Neiner C, Lignières F, Green JJ (eds) Studying stellar rotation and convection: theoretical background and seismic diagnostics, Lecture Notes in Physics, vol 865. Springer, Berlin, p 179. doi:10.1007/978-3-642-33380-4_9 
Belkacem K, Goupil MJ, Dupret MA, Samadi R, Baudin F, Noels A, Mosser B (2011) The underlying physical meaning of the $v_{\max }-v_{c}$ relation. Astron Astrophys 530:A142. doi:10.1051/0004-6361/ 201116490. arXiv:1104.0630

Benevolenskaya EE (1995) Double magnetic cycle of solar activity. Solar Phys 161:1-8. doi:10.1007/ BF00732080

Benevolenskaya EE (1998a) A model of the double magnetic cycle of the Sun. Astrophys J Lett 509:L49L52. doi:10.1086/311755. arXiv:astro-ph/9810329

Benevolenskaya EE (1998b) Longitudinal structure of the double magnetic cycle. Solar Phys 181:479-489. doi:10.1023/A:1005077029797

Bergemann M, Serenelli A (2014) Solar abundance problem. In: Niemczura E, Smalley B, Pych W (eds) Determination of atmospheric parameters of B-, A-, F- and G-type stars, GeoPlanet: Earth and Planetary Sciences. Springer, Cham. doi:10.1007/978-3-319-06956-2_21. arXiv:1403.3097

Bertello L, Varadi F, Ulrich RK, Henney CJ, Kosovichev AG, García RA, Turck-Chièze S (2000) Identification of solar acoustic modes of low angular degree and low radial order. Astrophys $\mathbf{J}$ Lett 537:L143-L146. doi:10.1086/312775

Berthomieu G, Provost J, Rocca A, Cooper AJ, Gough DO, Osaki Y (1980) Sensitivity of five minute eigenfrequencies to the structure of the sun. In: Hill HA, Dziembowski WA (eds) Nonradial and nonlinear stellar pulsation, Lecture Notes in Physics, vol 125. Springer, Berlin, pp 307-312. doi:10. 1007/3-540-09994-8_29

Biermann L (1948) Konvektion in rotierenden Sternen. Z Astrophys 25:135

Böhm-Vitense E (1958) Über die Wasserstoffkonvektionszone in Sternen verschiedener Effektivtemperaturen und Leuchtkräfte. Z Astrophys 46:108

Bouchy F, Carrier F (2001) P-mode observations on $\alpha$ Cen A. Astron Astrophys 374:L5-L8. doi:10.1051/ 0004-6361:20010792. arXiv:astro-ph/0107099

Brodsky M, Vorontsov SV (1993) Asymptotic theory of intermediate- and high-degree solar acoustic oscillations. Astrophys J 409:455-464. doi:10.1086/172678

Brodsky MA, Vorontsov SV (1987) An asymptotic technique for solving the inverse problem of helioseismology. Sov Astron Lett 13:179

Brodsky MA, Vorontsov SV (1988) On the technique of the inversion of helioseismological data. In: Christensen-Dalsgaard J, Frandsen S (eds) Advances in helio- and asteroseismology, IAU Symposium, vol 123. Reidel, Dordrecht, p 137

Broomhall AM, Fletcher ST, Salabert D, Basu S, Chaplin WJ, Elsworth Y, García RA, Jiménez A, New R (2011) Are short-term variations in solar oscillation frequencies the signature of a second solar dynamo? J Phys Conf Ser 271:012025. doi:10.1088/1742-6596/271/1/012025. arXiv:1012.4933

Brown TM, Christensen-Dalsgaard J (1998) Accurate determination of the solar photospheric radius. Astrophys J Lett 500:L195. doi:10.1086/311416. arXiv:astro-ph/9803131

Brown TM, Gilliland RL (1990) A search for solar-like oscillations in Alpha Centauri A. Astrophys J 350:839-845. doi:10.1086/168435

Brown TM, Gilliland RL, Noyes RW, Ramsey LW (1991) Detection of possible p-mode oscillations on Procyon. Astrophys J 368:599-609. doi:10.1086/169725

Brun AS, Turck-Chièze S, Morel P (1998) Standard Solar models in the light of new helioseismic constraints. I. The solar core. Astrophys J 506:913-925. doi:10.1086/306271. arXiv:astro-ph/9806272

Brun AS, Antia HM, Chitre SM, Zahn JP (2002) Seismic tests for solar models with tachocline mixing. Astron Astrophys 391:725-739. doi:10.1051/0004-6361:20020837. arXiv:astro-ph/0206180

Bruntt H, Bedding TR, Quirion PO, Lo Curto G, Carrier F, Smalley B, Dall TH, Arentoft T, Bazot M, Butler RP (2010) Accurate fundamental parameters for 23 bright solar-type stars. Mon Not R Astron Soc 405:1907-1923. doi:10.1111/j.1365-2966.2010.16575.x. arXiv:1002.4268

Buzasi D (2000) Platforms of opportunity: asteroseismology by Piggyback. In: Pallavicini R, Micela G, Sciortino S (eds) Stellar clusters and associations: convection, rotation, and dynamos, ASP Conference Series, vol 198. Astronomical Society of the Pacific, San Francisco, p 557

Buzasi D, Catanzarite J, Laher R, Conrow T, Shupe D, Gautier TN III, Kreidl T, Everett D (2000) The detection of multimodal oscillations on $\alpha$ ursae majoris. Astrophys J Lett 532:L133-L136. doi:10. 1086/312573. arXiv:astro-ph/0002512

Caffau E, Ludwig HG, Bonifacio P, Faraggiana R, Steffen M, Freytag B, Kamp I, Ayres TR (2010) The solar photospheric abundance of carbon. Analysis of atomic carbon lines with the CO5BOLD solar model. Astron Astrophys 514:A92. doi:10.1051/0004-6361/200912227. arXiv:1002.2628 
Caffau E, Ludwig HG, Steffen M, Freytag B, Bonifacio P (2011) Solar chemical abundances determined with a CO5BOLD 3D model atmosphere. Solar Phys 268:255-269. doi:10.1007/s11207-010-9541-4. arXiv: 1003.1190

Canuto VM (2011) Stellar mixing. V. Overshooting. Astron Astrophys 528:A80. doi:10.1051/0004-6361/ 201014450

Canuto VM, Mazzitelli I (1991) Stellar turbulent convection: a new model and applications. Astrophys J 370:295-311. doi:10.1086/169815

Carrier F, Eggenberger P, Bouchy F (2005a) New seismological results on the G0 IV $\eta$ Bootis. Astron Astrophys 434:1085-1095. doi:10.1051/0004-6361:20042140. arXiv:astro-ph/0501262

Carrier F, Eggenberger P, D'Alessandro A, Weber L (2005b) Solar-like oscillations in the F9 V $\beta$ Virginis. New Astron 10:315-323. doi:10.1016/j.newast.2004.11.003. arXiv:astro-ph/0502014

Castellani V, Degl'Innocenti S, Fiorentini G, Lissia M, Ricci B (1997) Solar neutrinos: beyond standard solar models. Phys Rep 281:309-398. doi:10.1016/S0370-1573(96)00032-4. arXiv:astro-ph/9606180

Castro M, Vauclair S, Richard O (2007) Low abundances of heavy elements in the solar outer layers: comparisons of solar models with helioseismic inversions. Astron Astrophys 463:755-758. doi:10. 1051/0004-6361:20066327. arXiv:astro-ph/0611619

Chandrasekhar S (1964) A general variational principle governing the radial and the non-radial oscillations of gaseous masses. Astrophys J 139:664. doi:10.1086/147792

Chaplin WJ, Miglio A (2013) Asteroseismology of solar-type and red-giant stars. Annu Rev Astron Astrophys 51:353-392. doi:10.1146/annurev-astro-082812-140938. arXiv:1303.1957

Chaplin WJ, Elsworth Y, Howe R, Isaak GR, McLeod CP, Miller BA, New R (1995) An investigation of the correlation between unusually large amplitude P-modes and solar activity. In: Hoeksema JT, Domingo V, Fleck B, Battrick B (eds) Helioseismology, ESA Special Publication, vol SP-376. ESA, Noordwijk, p 335

Chaplin WJ, Elsworth Y, Howe R, Isaak GR, McLeod CP, Miller BA, New R (1997) The observation and simulation of stochastically excited solar P modes. Mon Not R Astron Soc 287:51-56. doi:10.1093/ mnras/287.1.51

Chaplin WJ, Elsworth Y, Miller BA, Verner GA, New R (2007) Solar p-mode frequencies over three solar cycles. Astrophys J 659:1749-1760. doi:10.1086/512543

Chaplin WJ, Appourchaux T, Arentoft T, Ballot J, Christensen-Dalsgaard J, Creevey OL, Elsworth Y, Fletcher ST, García RA, Houdek G, Jiménez-Reyes SJ, Kjeldsen H, New R, Régulo C, Salabert D, Sekii T, Sousa SG, Toutain T, the rest of asteroFLAG Group (2008) AsteroFLAG: First results from hare-and-hounds Exercise \#1. Astron Nachr 329:549. doi:10.1002/asna.200710995. arXiv:0803.4143

Chaplin WJ, Appourchaux T, Elsworth Y, García RA, Houdek G, Karoff C, Metcalfe TS, Molenda-Zakowicz J, Monteiro MJPFG, Thompson MJ, Brown TM, Christensen-Dalsgaard J, Gilliland RL, Kjeldsen H, Borucki WJ, Koch D, Jenkins JM, Ballot J, Basu S, Bazot M, Bedding TR, Benomar O, Bonanno A, Brandão IM, Bruntt H, Campante TL, Creevey OL, Di Mauro MP, Doğan G, Dreizler S, Eggenberger P, Esch L, Fletcher ST, Frandsen S, Gai N, Gaulme P, Handberg R, Hekker S, Howe R, Huber D, Korzennik SG, Lebrun JC, Leccia S, Martic M, Mathur S, Mosser B, New R, Quirion PO, Régulo C, Roxburgh IW, Salabert D, Schou J, Sousa SG, Stello D, Verner GA, Arentoft T, Barban C, Belkacem K, Benatti S, Biazzo K, Boumier P, Bradley PA, Broomhall AM, Buzasi DL, Claudi RU, Cunha MS, D’Antona F, Deheuvels S, Derekas A, García Hernández A, Giampapa MS, Goupil MJ, Gruberbauer M, Guzik JA, Hale SJ, Ireland MJ, Kiss LL, Kitiashvili IN, Kolenberg K, Korhonen H, Kosovichev AG, Kupka F, Lebreton Y, Leroy B, Ludwig HG, Mathis S, Michel E, Miglio A, Montalbán J, Moya A, Noels A, Noyes RW, Pallé PL, Piau L, Preston HL, Roca Cortés T, Roth M, Sato KH, Schmitt J, Serenelli AM, Silva Aguirre V, Stevens IR, Suárez JC, Suran MD, Trampedach R, Turck-Chièze S, Uytterhoeven K, Ventura R, Wilson PA (2010) The Asteroseismic potential of Kepler: first results for solar-type stars. Astrophys J Lett 713:L169-L175. doi:10.1088/2041-8205/713/2/L169. arXiv:1001.0506

Chaplin WJ, Sanchis-Ojeda R, Campante TL, Handberg R, Stello D, Winn JN, Basu S, ChristensenDalsgaard J, Davies GR, Metcalfe TS, Buchhave LA, Fischer DA, Bedding TR, Cochran WD, Elsworth Y, Gilliland RL, Hekker S, Huber D, Isaacson H, Karoff C, Kawaler SD, Kjeldsen H, Latham DW, Lund MN, Lundkvist M, Marcy GW, Miglio A, Barclay T, Lissauer JJ (2013) Asteroseismic determination of obliquities of the exoplanet systems Kepler-50 and Kepler-65. Astrophys J 766:101. doi:10. 1088/0004-637X/766/2/101. arXiv:1302.3728

Chaplin WJ, Basu S, Huber D, Serenelli A, Casagrande L, Silva Aguirre V, Ball WH, Creevey OL, Gizon L, Handberg R, Karoff C, Lutz R, Marques JP, Miglio A, Stello D, Suran MD, Pricopi D, Metcalfe TS, Monteiro MJPFG, Molenda-Zakowicz J, Appourchaux T, Christensen-Dalsgaard J, Elsworth Y, 
García RA, Houdek G, Kjeldsen H, Bonanno A, Campante TL, Corsaro E, Gaulme P, Hekker S, Mathur S, Mosser B, Régulo C, Salabert D (2014) Asteroseismic fundamental properties of solar-type stars observed by the NASA Kepler mission. Astrophys J Suppl Ser 210:1. doi:10.1088/0067-0049/ 210/1/1. arXiv:1310.4001

Chou DY, Serebryanskiy A (2005) In search of the solar cycle variations of p-mode frequencies generated by perturbations in the solar interior. Astrophys J 624:420-427. doi:10.1086/428925. arXiv:astro-ph/0405175

Choubey S, Goswami S, Kar K, Antia HM, Chitre SM (2001) Global oscillation analysis of solar neutrino data with helioseismically constrained fluxes. Phys Rev D 64:113001. doi:10.1103/PhysRevD.64. 113001. arXiv:hep-ph/0106168

Christensen-Dalsgaard J (1982) On solar models and their periods of oscillation. Mon Not R Astron Soc 199:735-761. doi:10.1093/mnras/199.3.735

Christensen-Dalsgaard J (1988) A Hertzsprung-Russell diagram for stellar oscillations. In: ChristensenDalsgaard J, Frandsen S (eds) Advances in helio- and asteroseismology, IAU Symposium, vol 123. Reidel, Dordrecht, p 295

Christensen-Dalsgaard J (2002) Helioseismology. Rev Mod Phys 74:1073-1129. doi:10.1103/ RevModPhys.74.1073. arXiv:astro-ph/0207403

Christensen-Dalsgaard J (2003) Lecture Notes on Stellar Oscillations, 5th edn. University of Aarhus, Aarhus. http://astro.phys.au.dk/ jcd/oscilnotes/

Christensen-Dalsgaard J (2004) An overview of helio- and asteroseismology. In: Danesy D (ed) Helio- and asteroseismology: towards a golden future, ESA Special Publication, vol 559. ESA, Noordwijk, p 1

Christensen-Dalsgaard J (2016) Asteroseismology with solar-like oscillations. ArXiv e-prints arXiv: 1602.06838

Christensen-Dalsgaard J, Berthomieu G (1991) Theory of solar oscillations. In: Cox AN, Livingston WC, Matthews MS (eds) Solar interior and atmosphere, Space Science Series. University of Arizona Press, Tucson, pp 401-478

Christensen-Dalsgaard J, Däppen W (1992) Solar oscillations and the equation of state. Astron Astrophys Rev 4:267-361. doi:10.1007/BF00872687

Christensen-Dalsgaard J, Frandsen S (1983) Radiative transfer and solar oscillations. Solar Phys 82:165204. doi:10.1007/BF00145558

Christensen-Dalsgaard J, Gough DO (1975) Nonadiabatic nonradial oscillations of a solar model. Mém Soc R Sci Liège 8:309-316

Christensen-Dalsgaard J, Gough DO (1980) Is the sun helium-deficient. Nature 288:544-547. doi:10.1038/ $288544 \mathrm{a} 0$

Christensen-Dalsgaard J, Gough DO (1981) Comparison of observed solar whole-disk oscillation frequencies with the predictions of a sequence of solar models. Astron Astrophys 104:173-176

Christensen-Dalsgaard J, Gough DO (1982) On the interpretation of five-minute oscillations in solar spectrum line shifts. Mon Not R Astron Soc 198:141-171. doi:10.1093/mnras/198.1.141

Christensen-Dalsgaard J, Pérez Hernández F (1992) The phase function for stellar acoustic oscillations-I. Theory. Mon Not R Astron Soc 257:62-88. doi:10.1093/mnras/257.1.62

Christensen-Dalsgaard J, Thompson MJ (1997) On solar p-mode frequency shifts caused by near-surface model changes. Mon Not R Astron Soc 284:527-540. doi:10.1093/mnras/284.3.527

Christensen-Dalsgaard J, Gough DO, Morgan JG (1979) Dirty solar models. Astron Astrophys 73:121-128

Christensen-Dalsgaard J, Duvall TL Jr, Gough DO, Harvey JW, Rhodes EJ Jr (1985) Speed of sound in the solar interior. Nature 315:378-382. doi:10.1038/315378a0

Christensen-Dalsgaard J, Däppen W, Lebreton Y (1988a) Solar oscillation frequencies and the equation of state. Nature 336:634-638. doi:10.1038/336634a0

Christensen-Dalsgaard J, Gough DO, Pérez Hernández F (1988b) Stellar disharmony. Mon Not R Astron Soc 235:875-880. doi:10.1093/mnras/235.3.875

Christensen-Dalsgaard J, Gough DO, Thompson MJ (1988c) Determination of the solar internal sound speed by means of a differential asymptotic inversion. In: Rolfe EJ (ed) Seismology of the Sun and Sun-like stars, ESA Special Publication, vol 286. ESA, Noordwijk, pp 493-497

Christensen-Dalsgaard J, Gough DO, Libbrecht KG (1989a) Seismology of solar oscillation line widths. Astrophys J Lett 341:L103-L106. doi:10.1086/185468

Christensen-Dalsgaard J, Thompson MJ, Gough DO (1989b) Differential asymptotic sound-speed inversions. Mon Not R Astron Soc 238:481-502. doi:10.1093/mnras/238.2.481 
Christensen-Dalsgaard J, Schou J, Thompson MJ (1990) A comparison of methods for inverting helioseismic data. Mon Not R Astron Soc 242:353-369. doi:10.1093/mnras/242.3.353

Christensen-Dalsgaard J, Gough DO, Thompson MJ (1991) The depth of the solar convection zone. Astrophys J 378:413-437. doi:10.1086/170441

Christensen-Dalsgaard J, Proffitt CR, Thompson MJ (1993) Effects of diffusion on solar models and their oscillation frequencies. Astrophys J Lett 403:L75-L78. doi:10.1086/186725

Christensen-Dalsgaard J, Monteiro MJPFG, Thompson MJ (1995) Helioseismic estimation of convective overshoot in the Sun. Mon Not R Astron Soc 276:283-292

Christensen-Dalsgaard J, Däppen W, Ajukov SV, Anderson ER, Antia HM, Basu S, Baturin VA, Berthomieu G, Chaboyer B, Chitre SM, Cox AN, Demarque P, Donatowicz J, Dziembowski WA, Gabriel M, Gough DO, Guenther DB, Guzik JA, Harvey JW, Hill F, Houdek G, Iglesias CA, Kosovichev AG, Leibacher JW, Morel P, Proffitt CR, Provost J, Reiter J, Rhodes EJ Jr, Rogers FJ, Roxburgh IW, Thompson MJ, Ulrich RK (1996) The current state of solar modeling. Science 272:1286-1292. doi:10.1126/science. 272.5266.1286

Christensen-Dalsgaard J, Di Mauro MP, Schlattl H, Weiss A (2005) On helioseismic tests of basic physics. Mon Not R Astron Soc 356:587-595. doi:10.1111/j.1365-2966.2004.08477.x

Christensen-Dalsgaard J, di Mauro MP, Houdek G, Pijpers F (2009) On the opacity change required to compensate for the revised solar composition. Astron Astrophys 494:205-208. doi:10.1051/0004-6361: 200810170. arXiv:0811.1001

Claverie A, Isaak GR, McLeod CP, van der Raay HB, Cortes TR (1979) Solar structure from global studies of the 5-minute oscillation. Nature 282:591-594. doi:10.1038/282591a0

Coelho HR, Chaplin WJ, Basu S, Serenelli A, Miglio A, Reese DR (2015) A test of the asteroseismic $v_{\max }$ scaling relation for solar-like oscillations in main-sequence and sub-giant stars. Mon Not R Astron Soc 451:3011-3020. doi:10.1093/mnras/stv1175. arXiv:1505.06087

Cohen ER, Taylor BN (1987) The 1986 adjustment of the fundamental physical constants. Rev Mod Phys 59:1121-1148. doi:10.1103/RevModPhys.59.1121

Couvidat S, Turck-Chièze S, Kosovichev AG (2003) Solar seismic models and the neutrino predictions. Astrophys J 599:1434-1448. doi:10.1086/379604. arXiv:astro-ph/0203107

Cowling TG, Newing RA (1949) The oscillations of a rotating star. Astrophys J 109:149. doi:10.1086/ 145114

Cox AN, Tabor JE (1976) Radiative opacity tables for 40 stellar mixtures. Astrophys J Suppl Ser 31:271-312. doi:10.1086/190383

Cox AN, Guzik JA, Kidman RB (1989) Oscillations of solar models with internal element diffusion. Astrophys J 342:1187-1206. doi:10.1086/167675

Cox JP (1967) The linear theory: initiation of pulsational instability in stars. In: Thomas RN (ed) Aerodynamic phenomena in stellar atmospheres, IAU Symposium, vol 28. Academic Press, London, p 3

Cox JP (1980) Theory of stellar pulsations. Princeton University Press, Princeton

Craig IJD, Brown JC (1986) Inverse problems in astronomy: a guide to inversion strategies for remotely sensed data. Adam Hilger, Bristol

Cranmer SR, Hoeksema JT, Kohl JL (eds) (2010) SOHO-23: understanding a peculiar solar minimum, ASP Conference Series, vol 428. Astronomical Society of the Pacific, San Francisco

Creevey OL, Doğan G, Frasca A, Thygesen AO, Basu S, Bhattacharya J, Biazzo K, Brandão IM, Bruntt H, Mazumdar A, Niemczura E, Shrotriya T, Sousa SG, Stello D, Subramaniam A, Campante TL, Handberg R, Mathur S, Bedding TR, García RA, Régulo C, Salabert D, Molenda-Zakowicz J, Quirion PO, White TR, Bonanno A, Chaplin WJ, Christensen-Dalsgaard J, Christiansen JL, Elsworth Y, Fanelli MN, Karoff C, Kinemuchi K, Kjeldsen H, Gai N, Monteiro MJPFG, Suárez JC (2012) Fundamental properties of five Kepler stars using global asteroseismic quantities and ground-based observations. Astron Astrophys 537:A111. doi:10.1051/0004-6361/201117037. arXiv:1111.4615

Creevey OL, Thévenin F, Basu S, Chaplin WJ, Bigot L, Elsworth Y, Huber D, Monteiro MJPFG, Serenelli A (2013) A large sample of calibration stars for Gaia: $\log g$ from Kepler and CoRoT fields. Mon Not R Astron Soc 431:2419-2432. doi:10.1093/mnras/stt336. arXiv:1302.7158

Däppen W, Gough DO (1986) Progress report on helium abundance determination. In: Gough DO (ed) Seismology of the Sun and the distant stars, NATO ASI Series C, vol 169. Reidel, Dordrecht, pp $275-280$ 
Däppen W, Gough DO, Thompson MJ (1988) Further progress on the helium abundance determination. In: Rolfe EJ (ed) Seismology of the Sun and sun-like stars, ESA Special Publication, vol 286. ESA, Noordwijk, pp 505-510

Däppen W, Mihalas D, Hummer DG, Mihalas BW (1988) The equation of state for stellar envelopes. III. Thermodynamic quantities. Astrophys J 332:261-270. doi:10.1086/166650

Däppen W, Gough DO, Kosovichev AG, Thompson MJ (1991) A new inversion for the hydrostatic stratification of the Sun. In: Gough D, Toomre J (eds) Challenges to theories of the structure of moderate-mass stars, Lecture Notes in Physics, vol 388. Springer, Berlin, p 111. doi:10.1007/3-540-54420-8_57

Davies GR, Chaplin WJ, Farr WM, García RA, Lund MN, Mathis S, Metcalfe TS, Appourchaux T, Basu S, Benomar O, Campante TL, Ceillier T, Elsworth Y, Handberg R, Salabert D, Stello D (2015) Asteroseismic inference on rotation, gyrochronology and planetary system dynamics of 16 Cygni. Mon Not R Astron Soc 446:2959-2966. doi:10.1093/mnras/stu2331. arXiv:1411.1359

Davis $\mathrm{R}$ (1955) Attempt to detect the antineutrinos from a nuclear reactor by the $\mathrm{Cl}^{37}\left(\bar{v}, e^{-}\right) \mathrm{A}^{37}$ reaction. Phys Rev 97:766-769. doi:10.1103/PhysRev.97.766

Davis R (1964) Solar neutrinos. II. Experimental. Phys Rev Lett 12:303-305. doi:10.1103/PhysRevLett. 12.303

Davis R (1994) A review of the homestake solar neutrino experiment. Prog Part Nucl Phys 32:13-32. doi:10. 1016/0146-6410(94)90004-3

de Boor C (2001) A practical guide to splines, applied mathematical sciences, vol 27, rev edn. Springer, New York

De Ridder J, Barban C, Baudin F, Carrier F, Hatzes AP, Hekker S, Kallinger T, Weiss WW, Baglin A, Auvergne M, Samadi R, Barge P, Deleuil M (2009) Non-radial oscillation modes with long lifetimes in giant stars. Nature 459:398-400. doi:10.1038/nature08022

Degl'Innocenti S, Fiorentini G, Ricci B (1998) Helioseismology and $\mathrm{p}+\mathrm{p} \rightarrow \mathrm{d}+\mathrm{e}^{+}+v_{e}$ in the sun. Phys Lett B 416:365-368. doi:10.1016/S0370-2693(97)01197-0. arXiv:astro-ph/9707133

Deheuvels S, the CoRoT Team (2014) Some highlights of the latest CoRoT results on stellar physics. In: Guzik JA, Chaplin WJ, Handler G, Pigulski A (eds) Precision asteroseismology, IAU Symposium, vol 301. Cambridge University Press, Cambridge, pp 47-54. doi:10.1017/S1743921313014063

Deheuvels S, Michel E (2011) Constraints on the structure of the core of subgiants via mixed modes: the case of HD 49385. Astron Astrophys 535:A91. doi:10.1051/0004-6361/201117232. arXiv:1109.1191

Deheuvels S, Bruntt H, Michel E, Barban C, Verner G, Régulo C, Mosser B, Mathur S, Gaulme P, Garcia RA, Boumier P, Appourchaux T, Samadi R, Catala C, Baudin F, Baglin A, Auvergne M, Roxburgh IW, Pérez Hernández F (2010) Seismic and spectroscopic characterization of the solar-like pulsating CoRoT target HD 49385. Astron Astrophys 515:A87. doi:10.1051/0004-6361/200913490. arXiv:1003.4368

Deheuvels S, García RA, Chaplin WJ, Basu S, Antia HM, Appourchaux T, Benomar O, Davies GR, Elsworth Y, Gizon L, Goupil MJ, Reese DR, Régulo C, Schou J, Stahn T, Casagrande L, Christensen-Dalsgaard J, Fischer D, Hekker S, Kjeldsen H, Mathur S, Mosser B, Pinsonneault M, Valenti J, Christiansen JL, Kinemuchi K, Mullally F (2012) Seismic evidence for a rapidly rotating core in a lower-giant-branch star observed with Kepler. Astrophys J 756:19. doi:10.1088/0004-637X/756/1/19. arXiv:1206.3312

Deheuvels S, Doğan G, Goupil MJ, Appourchaux T, Benomar O, Bruntt H, Campante TL, Casagrande L, Ceillier T, Davies GR, De Cat P, Fu JN, García RA, Lobel A, Mosser B, Reese DR, Régulo C, Schou J, Stahn T, Thygesen AO, Yang XH, Chaplin WJ, Christensen-Dalsgaard J, Eggenberger P, Gizon L, Mathis S, Molenda-Zakowicz J, Pinsonneault M (2014) Seismic constraints on the radial dependence of the internal rotation profiles of six Kepler subgiants and young red giants. Astron Astrophys 564:A27. doi:10.1051/0004-6361/201322779. arXiv:1401.3096

Demarque P, Krauss LM, Guenther DB, Nydam D (1994) The Sun as a probe of varying G. Astrophys J 437:870-878. doi:10.1086/175048

Demarque P, Guenther DB, Kim YC (1997) The run of superadiabaticity in stellar convection zones. I. The Sun. Astrophys J 474:790. doi:10.1086/303477

Demarque PR, Percy JR (1964) A series of solar models. Astrophys J 140:541. doi:10.1086/147947

Deubner FL (1975) Observations of low wavenumber nonradial eigenmodes of the Sun. Astron Astrophys 44:371-375

Deubner FL, Gough D (1984) Helioseismology: oscillations as a daignostic of the solar interior. Annu Rev Astron Astrophys 22:593-619. doi:10.1146/annurev.aa.22.090184.003113

Di Mauro MP, Christensen-Dalsgaard J, Rabello-Soares MC, Basu S (2002) Inferences on the solar envelope with high-degree modes. Astron Astrophys 384:666-677. doi:10.1051/0004-6361:20020020 
Domingo V, Fleck B, Poland AI (1995) The SOHO mission: an overview. Solar Phys 162:1-37. doi:10. 1007/BF00733425

Dupret MA, Belkacem K, Samadi R, Montalban J, Moreira O, Miglio A, Godart M, Ventura P, Ludwig HG, Grigahcène A, Goupil MJ, Noels A, Caffau E (2009) Theoretical amplitudes and lifetimes of non-radial solar-like oscillations in red giants. Astron Astrophys 506:57-67. doi:10.1051/0004-6361/ 200911713. arXiv:0906.3951

Duvall TL Jr (1980) The equatorial rotation rate of the supergranulation cells. Solar Phys 66:213-221. doi:10.1007/BF00150578

Duvall TL Jr (1982) A dispersion law for solar oscillations. Nature 300:242. doi:10.1038/300242a0

Duvall TL Jr, Harvey JW (1983) Observations of solar oscillations of low and intermediate degree. Nature 302:24-27. doi:10.1038/302024a0

Dziembowski W (1982) Nonlinear mode coupling in oscillating stars. I. Second order theory of the coherent mode coupling. Acta Astron 32:147-171

Dziembowski WA, Goode PR (1989) The toroidal magnetic field inside the sun. Astrophys J 347:540-550. doi:10.1086/168144

Dziembowski WA, Goode PR (1991) Seismology for the fine structure in the sun's oscillations varying with its activity cycle. Astrophys J 376:782-786. doi:10.1086/170326

Dziembowski WA, Goode PR (2005) Sources of oscillation frequency increase with rising solar activity. Astrophys J 625:548-555. doi:10.1086/429712. arXiv:astro-ph/0503266

Dziembowski WA, Pamyatnykh AA, Sienkiewicz R (1990) Solar model from helioseismology and the neutrino flux problem. Mon Not R Astron Soc 244:542-550

Dziembowski WA, Pamiatnykh AA, Sienkiewicz R (1991) Helium content in the solar convective envelope from helioseismology. Mon Not R Astron Soc 249:602-605. doi:10.1093/mnras/249.4.602

Dziembowski WA, Goode PR, Pamyatnykh AA, Sienkiewicz R (1994) A seismic model of the Sun's interior. Astrophys J 432:417-426. doi:10.1086/174580

Dziembowski WA, Goode PR, Pamyatnykh AA, Sienkiewicz R (1995) Updated seismic solar model. Astrophys J 445:509-510. doi:10.1086/175715

Dziembowski WA, Goode PR, di Mauro MP, Kosovichev AG, Schou J (1998) Solar cycle onset seen in SOHO Michelson doppler imager seismic data. Astrophys J 509:456-460. doi:10.1086/306498

Dziembowski WA, Goode PR, Kosovichev AG, Schou J (2000) Signatures of the rise of cycle 23. Astrophys J 537:1026-1038. doi:10.1086/309056

Edmonds PD, Cram LE (1995) A search for global acoustic oscillations on $\alpha^{1}$ Cen and $\beta$ Hyi. Mon Not R Astron Soc 276:1295-1302

Eff-Darwich A, Korzennik SG (2013) The dynamics of the solar radiative zone. Solar Phys 287:43-56. doi:10.1007/s11207-012-0048-z. arXiv:1205.3143

Eff-Darwich A, Korzennik SG, Jiménez-Reyes SJ, Pérez Hernández F (2002) An upper limit on the temporal variations of the solar interior stratification. Astrophys J 580:574-578. doi:10.1086/343063. arXiv:astro-ph/0207402

Eggleton PP, Faulkner J, Flannery BP (1973) An approximate equation of state for stellar material. Astron Astrophys 23:325

Elliott JR (1996) Equation of state in the solar convection zone and the implications of helioseismology. Mon Not R Astron Soc 280:1244-1256. doi:10.1093/mnras/280.4.1244

Elliott JR, Kosovichev AG (1998) The adiabatic exponent in the solar core. Astrophys J Lett 500:L199. doi:10.1086/311417

Elsworth Y, Howe R, Isaak GR, McLeod CP, New R (1990a) Evidence from solar seismology against non-standard solar-core models. Nature 347:536-539. doi:10.1038/347536a0

Elsworth Y, Howe R, Isaak GR, McLeod CP, New R (1990b) Variation of low-order acoustic solar oscillations over the solar cycle. Nature 345:322-324. doi:10.1038/345322a0

Elsworth Y, Howe R, Isaak GR, McLeod CP, New R (1991) Low- $\ell$ p-mode solar eigenfrequency measurements from the Birmingham Network. Mon Not R Astron Soc 251:7P-9P. doi:10.1093/mnras/251.1. $7 \mathrm{P}$

Elsworth Y, Howe R, Isaak GR, McLeod CP, Miller BA, New R, Speake CC, Wheeler SJ (1994) Solar p-mode frequencies and their dependence on solar activity recent results from the BiSON network. Astrophys J 434:801-806. doi:10.1086/174783

Elsworth Y, Howe R, Isaak GR, McLeod CP, Miller BA, Wheeler SJ, New R (1995a) Fine Spacing of $\ell=0, \ell=2$ acoustic eigenmodes and the solar neutrino problem: particle physics and cosmological implications. In: Ulrich RK, Rhodes EJ Jr, Däppen W (eds) GONG '94: Helio- and astro-seismology 
from the earth and space, ASP Conference Series, vol 76. Astronomical Society of the Pacific, San Francisco, p 51

Elsworth Y, Howe R, Isaak GR, McLeod CP, Miller BA, Wheeler SJ, New R (1995b) Statistical distribution of solar p-mode amplitudes. In: Ulrich RK, Rhodes EJ Jr, Däppen W (eds) GONG '94: Helio- and astro-seismology from the earth and space, ASP Conference Series, vol 76. Astronomical Society of the Pacific, San Francisco, p 318

Epstein I, Motz L (1953) On the Sun's interior. I. Astrophys J 117:311. doi:10.1086/145696

Evans DJ, Roberts B (1992) Interpretation of solar-cycle variability in high-degree p-mode frequencies. Nature 355:230-232. doi:10.1038/355230a0

Evans JW, Michard R (1962) Observational study of macroscopic inhomogeneities in the solar atmosphere. III. Vertical oscillatory motions in the solar photosphere. Astrophys J 136:493. doi:10.1086/147403

Ezer D, Cameron AGW (1965) Solar evolution with varying G. Astron J 70:675. doi:10.1086/109618

Ferguson JW, Alexander DR, Allard F, Barman T, Bodnarik JG, Hauschildt PH, Heffner-Wong A, Tamanai A (2005) Low-temperature opacities. Astrophys J 623:585-596. doi:10.1086/428642. arXiv:astro-ph/0502045

Fletcher ST, Chaplin WJ, Elsworth Y, Schou J, Buzasi D (2006) Frequency, splitting, linewidth and amplitude estimates of low $\ell$ p modes of $\alpha$ Cen A: analysis of Wide-Field Infrared Explorer photometry. Mon Not R Astron Soc 371:935-944. doi:10.1111/j.1365-2966.2006.10727.x. arXiv:astro-ph/0607172

Fletcher ST, Broomhall AM, Salabert D, Basu S, Chaplin WJ, Elsworth Y, Garcia RA, New R (2010) A seismic signature of a second dynamo? Astrophys J Lett 718:L19-L22. doi:10.1088/2041-8205/718/ 1/L19. arXiv:1006.4305

Fossat E (1991) The IRIS network for full disk helioseismology: present status of the programme. Solar Phys 133:1-12. doi:10.1007/BF00149818

Frazier EN (1968) A spatio-temporal analysis of velocity fields in the solar photosphere. Z Astrophys 68:345

Fröhlich C, Lean J (1998) The Sun's total irradiance: cycles, trends and related climate change uncertainties since 1976. Geophys Rev Lett 25:4377-4380. doi:10.1029/1998GL900157

Gabriel AH, Charra J, Grec G, Robillot JM, Roca Cortés T, Turck-Chièze S, Ulrich R, Basu S, Baudin F, Bertello L, Boumier P, Charra M, Christensen-Dalsgaard J, Decaudin M, Dzitko H, Foglizzo T, Fossat E, García RA, Herreros JM, Lazrek M, Pallé PL, Pétrou N, Renaud C, Régulo C (1997) Performance and early results from the GOLF instrument flown on the SOHO mission. Solar Phys 175:207-226. doi:10.1023/A:1004911408285

Gai N, Basu S, Chaplin WJ, Elsworth Y (2011) An in-depth study of grid-based asteroseismic analysis. Astrophys J 730:63. doi:10.1088/0004-637X/730/2/63. arXiv:1009.3018

García RA (2010) Detecting individual gravity modes in the Sun: chimera or reality? Highlights Astron 15:345-346. doi:10.1017/S1743921310009695. arXiv:0911.5716

García RA, Turck-Chièze S, Jiménez-Reyes SJ, Ballot J, Pallé PL, Eff-Darwich A, Mathur S, Provost J (2007) Tracking solar gravity modes: the dynamics of the solar core. Science 316:1591. doi:10.1126/ science. 1140598

García RA, Jiménez A, Mathur S, Ballot J, Eff-Darwich A, Jiménez-Reyes SJ, Pallé PL, Provost J, TurckChièze S (2008) Update on g-mode research. Astron Nachr 329:476. doi:10.1002/asna.200710980. arXiv:0802.4296

Gilliland RL, Brown TM, Christensen-Dalsgaard J, Kjeldsen H, Aerts C, Appourchaux T, Basu S, Bedding TR, Chaplin WJ, Cunha MS, De Cat P, De Ridder J, Guzik JA, Handler G, Kawaler S, Kiss L, Kolenberg K, Kurtz DW, Metcalfe TS, Monteiro MJPFG, Szabó R, Arentoft T, Balona L, Debosscher J, Elsworth YP, Quirion PO, Stello D, Suárez JC, Borucki WJ, Jenkins JM, Koch D, Kondo Y, Latham DW, Rowe JF, Steffen JH (2010) Kepler asteroseismology program: introduction and first results. Publ Astron Soc Pac 122:131-143. doi:10.1086/650399. arXiv:1001.0139

Gilman PA, Howe R (2003) Meridional motion and the slope of isorotation contours. In: Sawaya-Lacoste H (ed) Local and global helioseismology: the present and future, ESA Special Publication, vol SP-517. ESA, Noordwijk, pp 283-285

Gizon L, Birch AC (2005) Local helioseismology. Living Rev Solar Phys 2:1rsp-2005-6. doi:10.12942/ lrsp-2005-6. http://www.livingreviews.org/lrsp-2005-6

Gizon L, Solanki SK (2003) Determining the inclination of the rotation axis of a Sun-like star. Astrophys J 589:1009-1019. doi:10.1086/374715

Gizon L, Solanki SK (2004) Measuring stellar differential rotation with asteroseismology. Solar Phys 220:169-184. doi:10.1023/B:SOLA.0000031378.29215.0c

Goldreich P, Keeley DA (1977a) Solar oscillations. Comments Astrophys 7:35-41 
Goldreich P, Keeley DA (1977b) Solar seismology. I. The stability of the solar p-modes. Astrophys J 211:934-942. doi:10.1086/155005

Goldreich P, Keeley DA (1977c) Solar seismology. II. The stochastic excitation of the solar p-modes by turbulent convection. Astrophys J 212:243-251. doi:10.1086/155043

Goldreich P, Kumar P (1988) The interaction of acoustic radiation with turbulence. Astrophys J 326:462478. doi:10.1086/166108

Goldreich P, Murray N (1994) The effects of scattering on solar oscillations. Astrophys J 424:480-490. doi:10.1086/173905

Goldreich P, Murray N, Willette G, Kumar P (1991) Implications of solar p-mode frequency shifts. Astrophys J 370:752-762. doi:10.1086/169858

Goldreich P, Murray N, Kumar P (1994) Excitation of solar p-modes. Astrophys J 424:466-479. doi:10. 1086/173904

Golub GH, Van Loan CF (1996) Matrix computations, 3rd edn. Johns Hopkins University Press, Baltimore

Gong Z, Däppen W, Zejda L (2001) MHD equation of state with relativistic electrons. Astrophys J 546:11781182. doi: $10.1086 / 318307$

Goode P, Strous L, Rimmele T, Stein R, Nordlund Å (1999) The excitation of solar oscillations: observations and simulations. In: Rimmele TR, Balasubramaniam KS, Radick RR (eds) High resolution solar physics: theory, observations, and techniques, ASP Conference Series, vol 183. Astronomical Society of the Pacific, San Francisco, p 456

Goode PR, Dziembowski WA (1993) Seismic limits on the Sun's internal toroidal field. In: Brown TM (ed) GONG 1992: Seismic investigation of the Sun and stars, ASP Conference Series, vol 42. Astronomical Society of the Pacific, San Franciso, p 229

Goode PR, Thompson MJ (1992) The effect of an inclined magnetic field on solar oscillation frequencies. Astrophys J 395:307-315. doi:10.1086/171653

Goode PR, Gough D, Kosovichev AG (1992) Localized excitation of solar oscillations. Astrophys J 387:707711. doi:10.1086/171118

Goode PR, Dziembowski WA, DiMauro MP, Kosovichev AG, Schou J (1998) Solar asymmetries from SOHO/MDI splitting data. In: Korzennik S, Wilson A (eds) Structure and dynamics of the interior of the Sun and Sun-like stars, ESA Special Publication, vol SP-418. ESA, Noordwijk, p 887

Gopalswamy N, Yashiro S, Mäkelä P, Michalek G, Shibasaki K, Hathaway DH (2012) Behavior of solar cycles 23 and 24 revealed by microwave observations. Astrophys J Lett 750:L42. doi:10.1088/ 2041-8205/750/2/L42. arXiv:1204.2816

Gough DO (1977a) Mixing-length theory for pulsating stars. Astrophys J 214:196-213. doi:10.1086/155244

Gough DO (1977b) Mixing-length theory for pulsating stars. Astrophys J 214:196-213. doi:10.1086/155244

Gough DO (1980) Some theoretical remarks on solar oscillations. In: Hill HA, Dziembowski WA (eds) Nonradial and nonlinear stellar pulsation, New York, Lecture Notes in Physics, vol 125. Springer, Berlin, pp 273-299. doi:10.1007/3-540-09994-8_27

Gough DO (1984) On the rotation of the Sun. Philos Trans R Soc Lond Ser A 313:27-38. doi:10.1098/rsta. 1984.0080

Gough DO (1984b) Towards a solar model. Mem Soc Astron It 55:13

Gough DO (1985) Inverting helioseismic data. Solar Phys 100:65-99. doi:10.1007/BF00158422

Gough DO (1990) Comments on helioseismic inference. In: Osaki Y, Shibahashi H (eds) Progress of seismology of the Sun and stars, Lecture Notes in Physics, vol 367. Springer, Berlin, p 283. doi:10. 1007/3-540-53091-6

Gough DO (1993) Linear adiabatic stellar pulsation. In: Zahn JP, Zinn-Justin J (eds) Astrophysical fluid dynamics (Dynamique des fluides astrophysiques). Elsevier, Amsterdam, pp 399-560

Gough DO (2002) How is solar activity influencing the structure of the Sun. In: Wilson A (ed) From solar min to max: half a solar cycle with SOHO, ESA Special Publication, vol 508. ESA, Noordwijk, pp 577-592

Gough DO (2013a) Commentary on a putative magnetic field variation in the solar convection zone. Mon Not R Astron Soc 435:3148-3158. doi:10.1093/mnras/stt1513

Gough DO (2013b) What have we learned from helioseismology, what have we really learned, and what do we aspire to learn? Solar Phys 287:9-41. doi:10.1007/s11207-012-0099-1. arXiv:1210.0820

Gough DO, Kosovichev AG (1988) An attempt to understand the Stanford p-mode data. In: Rolfe EJ (ed) Seismology of the Sun and Sun-like stars, ESA Special Publication, vol 286. ESA, Noordwijk, pp 195-201 
Gough DO, Kosovichev AG (1993) The influence of low-degree P-mode frequencies on the determination of the structure of the solar interior. Mon Not R Astron Soc 264:522. doi:10.1093/mnras/264.2.522

Gough DO, Kosovichev AG (1995) An attempt to measure latitudinal variation of the depth of the convection zone. In: Hoeksema JT, Domingo V, Fleck B, Battrick B (eds) Helioseismology, ESA Special Publication, vol SP-376. ESA, Noordwijk, pp 47-48

Gough DO, Sekii T (1993) on the detection of convective overshoot. In: Brown TM (ed) GONG 1992: Seismic investigation of the Sun and stars, ASP Conference Series, vol 42. Astronomical Society of the Pacific, San Franciso, p 177

Gough DO, Taylor PP (1984) Influence of rotation and magnetic fields on stellar oscillation eigenfrequencies. Mem Soc Astron It 55:215-226

Gough DO, Thompson MJ (1988) On the implications of the symmetric component of the frequency splitting. In: Christensen-Dalsgaard J, Frandsen S (eds) Advances in helio- and asteroseismology, IAU Symposium, vol 123. Reidel, Dordrecht, p 175

Gough DO, Thompson MJ (1990) The effect of rotation and a buried magnetic field on stellar oscillations. Mon Not R Astron Soc 242:25-55. doi:10.1093/mnras/242.1.25

Gough DO, Thompson MJ (1991) The inversion problem. In: Cox AN, Livingston WC, Matthews MS (eds) Solar interior and atmosphere. University of Arizona Press, Tucson, pp 519-561

Gough DO, Vorontsov SV (1995) Seismology of the solar envelope: measuring the acoustic phase shift generated in the outer layers. Mon Not R Astron Soc 273:573-582

Gough DO, Kosovichev AG, Sekii T, Libbrecht KG, Woodard MF (1993) Seismic evidence of modulation of the structure and angular velocity of the Sun associated with the solar cycle. In: Weiss WW, Baglin A (eds) Inside the stars: IAU Colloquium 137, ASP Conference Series, vol 40. Astronomical Society of the Pacific, San Francisco, p 93

Gough DO, Kosovichev AG, Toomre J, Anderson E, Antia HM, Basu S, Chaboyer B, Chitre SM, Christensen-Dalsgaard J, Dziembowski WA, Eff-Darwich A, Elliott JR, Giles PM, Goode PR, Guzik JA, Harvey JW, Hill F, Leibacher JW, Monteiro MJPFG, Richard O, Sekii T, Shibahashi H, Takata M, Thompson MJ, Vauclair S, Vorontsov SV (1996) The seismic structure of the Sun. Science 272:12961300. doi:10.1126/science.272.5266.1296

Grec G, Fossat E, Pomerantz MA (1983) Full-disk observations of solar oscillations from the geographic South Pole: latest results. Solar Phys 82:55-66. doi:10.1007/BF00145545

Grevesse N, Noels A (1993) Cosmic abundances of the elements. In: Prantzos N, Vangioni-Flam E, Casse M (eds) Origin and evolution of the elements. Cambridge University Press, Cambridge, pp 15-25

Grevesse N, Sauval AJ (1998) Standard solar composition. Space Sci Rev 85:161-174. doi:10.1023/A: 1005161325181

Gruberbauer M, Guenther DB, Kallinger T (2012) Toward a new kind of asteroseismic grid fitting. Astrophys J 749:109. doi:10.1088/0004-637X/749/2/109. arXiv:1202.2330

Guenther DB (1994) Nonadiabatic nonradial p-mode frequencies of the standard solar model, with and without helium diffusion. Astrophys J 422:400-411. doi:10.1086/173735

Guenther DB, Demarque P (1997) Seismic tests of the Sun's interior structure, composition, and age, and implications for solar neutrinos. Astrophys J 484:937. doi:10.1086/304367

Guenther DB, Jaffe A, Demarque P (1989) The standard solar model: composition, opacities, and seismology. Astrophys J 345:1022-1033. doi:10.1086/167972

Guenther DB, Demarque P, Kim YC, Pinsonneault MH (1992) Standard solar model. Astrophys J 387:372393. doi:10.1086/171090

Guenther DB, Sills K, Demarque P, Krauss LM (1995) Sensitivity of solar g-modes to varying G cosmologies. Astrophys J 445:148-151. doi:10.1086/175680

Guenther DB, Kim YC, Demarque P (1996) Seismology of the standard solar model: tests of diffusion and the OPAL and MHD equations of state. Astrophys J 463:382. doi:10.1086/177252

Guzik JA, Cox AN (1991) Effects of opacity and equation of state on solar structure and oscillations. Astrophys J 381:333-340. doi:10.1086/170655

Guzik JA, Cox AN (1992) On the sensitivity of high-degree p-mode frequencies to the solar convection zone helium abundance. Astrophys J 386:729-733. doi:10.1086/171054

Guzik JA, Cox AN (1993) Using solar p-modes to determine the convection zone depth and constrain diffusion-produced composition gradients. Astrophys J 411:394-401. doi:10.1086/172840

Guzik JA, Swenson FJ (1997) Seismological comparisons of solar models with element diffusion using the MHD, OPAL, and SIREFF equations of state. Astrophys J 491:967. doi:10.1086/304988 
Guzik JA, Willson LA, Brunish WM (1987) A comparison between mass-losing and standard solar models. Astrophys J 319:957-965. doi:10.1086/165512

Guzik JA, Watson LS, Cox AN (2005) Can enhanced diffusion improve helioseismic agreement for solar models with revised abundances? Astrophys J 627:1049-1056. doi:10.1086/430438. arXiv:astro-ph/0502364

Haberreiter M, Schmutz W, Kosovichev AG (2008) Solving the discrepancy between the seismic and photospheric solar radius. Astrophys J Lett 675:L53. doi:10.1086/529492

Hansen CJ, Kawaler SD, Trimble V (2004) Stellar interiors: physical principles, structure, and evolution, 2nd edn. Astronomy and Astrophysics Library, Springer, New York. doi:10.1007/978-1-4419-9110-2

Hansen PC (1992) Numerical tools for analysis and solution of Fredholm integral equations of the first kind. Inverse Prob 8:849-872. doi:10.1088/0266-5611/8/6/005

Hata N, Bludman S, Langacker P (1994) Astrophysical solutions are incompatible with the solar neutrino data. Phys Rev D 49:3622-3625. doi:10.1103/PhysRevD.49.3622. arXiv:hep-ph/9306212

Hathaway DH (2015) The solar cycle. Living Rev Solar Phys 12:1rsp-2015-4. doi:10.1007/1rsp-2015-4. http://www.livingreviews.org/lrsp-2015-4. arXiv:1502.07020

Haxton WC (1995) The solar neutrino problem. Annu Rev Astron Astrophys 33:459-504. doi:10.1146/ annurev.aa.33.090195.002331. arXiv:hep-ph/9503430

Heeger KM, Robertson RGH (1996) Probability of a solution to the solar neutrino problem within the minimal standard model. Phys Rev Lett 77:3720-3723. doi:10.1103/PhysRevLett.77.3720. arXiv:nucl-th/9610030

Hekker S, Elsworth Y, De Ridder J, Mosser B, García RA, Kallinger T, Mathur S, Huber D, Buzasi DL, Preston HL, Hale SJ, Ballot J, Chaplin WJ, Régulo C, Bedding TR, Stello D, Borucki WJ, Koch DG, Jenkins J, Allen C, Gilliland RL, Kjeldsen H, Christensen-Dalsgaard J (2011) Solar-like oscillations in red giants observed with Kepler: comparison of global oscillation parameters from different methods. Astron Astrophys 525:A131. doi:10.1051/0004-6361/201015185. arXiv:1008.2959

Hekker S, Elsworth Y, Basu S, Mazumdar A, Silva Aguirre V, Chaplin WJ (2013) Tests of the asymptotic large frequency separation of acoustic oscillations in solar-type and red-giant stars. Mon Not R Astron Soc 434:1668-1673. doi:10.1093/mnras/stt1238. arXiv:1306.4323

Henyey LG, Wilets L, Böhm KH, Lelevier R, Levee RD (1959) A method for automatic computation of stellar evolution. Astrophys J 129:628. doi:10.1086/146661

Hill F, Stark PB, Stebbins RT, Anderson ER, Antia HM, Brown TM, Duvall TL Jr, Haber DA, Harvey JW, Hathaway DH, Howe R, Hubbard RP, Jones HP, Kennedy JR, Korzennik SG, Kosovichev AG, Leibacher JW, Libbrecht KG, Pintar JA, Rhodes EJ Jr, Schou J, Thompson MJ, Tomczyk S, Toner CG, Toussaint R, Williams WE (1996) The solar acoustic spectrum and eigenmode parameters. Science 272:1292-1295. doi:10.1126/science.272.5266.1292

Houdek G, Balmforth NJ, Christensen-Dalsgaard J, Gough DO (1999) Amplitudes of stochastically excited oscillations in main-sequence stars. Astron Astrophys 351:582-596 arXiv:astro-ph/9909107

Howard R, Labonte BJ (1980) The sun is observed to be a torsional oscillator with a period of 11 years. Astrophys J Lett 239:L33-L36. doi:10.1086/183286

Howe R (2009) Solar interior rotation and its variation. Living Rev Solar Phys 6:1rsp-2009-1. doi:10.12942/ lrsp-2009-1. http://www.livingreviews.org/lrsp-2009-1. arXiv:0902.2406

Howe R, Thompson MJ (1996) On the use of the error correlation function in helioseismic inversions. Mon Not R Astron Soc 281:1385. doi:10.1093/mnras/281.4.1385

Howe R, Komm R, Hill F (1999) Solar cycle changes in GONG p-mode frequencies, 1995-1998. Astrophys J 524:1084-1095. doi:10.1086/307851

Howe R, Christensen-Dalsgaard J, Hill F, Komm RW, Larsen RM, Schou J, Thompson MJ, Toomre J (2000) Deeply penetrating banded zonal flows in the solar convection zone. Astrophys J Lett 533:L163-L166. doi:10.1086/312623. arXiv:astro-ph/0003121

Howe R, Komm RW, Hill F (2002) Localizing the solar cycle frequency shifts in global p-modes. Astrophys J 580:1172-1187. doi:10.1086/343892

Howe R, Christensen-Dalsgaard J, Hill F (2005) Solar convection-zone dynamics, 1995-2004. Astrophys J 634:1405-1415. doi:10.1086/497107

Howe R, Chaplin WJ, Elsworth Y, Hill F, Komm RW, Isaak GR, New R (2006) Anomalous variations in low-degree helioseismic mode frequencies. Mon Not R Astron Soc 369:933-938. doi:10.1111/j. 1365-2966.2006.10346.x 
Howe R, Rempel M, Christensen-Dalsgaard J, Hill F, Komm R, Larsen RM, Schou J, Thompson MJ (2006b) Solar convection zone dynamics: how sensitive are inversions to subtle dynamo features? Astrophys J 649:1155-1168. doi:10.1086/506931

Howe R, Christensen-Dalsgaard J, Hill F, Komm R, Schou J, Thompson MJ (2009) A note on the torsional oscillation at solar minimum. Astrophys J Lett 701:L87-L90. doi:10.1088/0004-637X/701/2/L87. arXiv:0907.2965

Howe R, Hill F, Komm R, Christensen-Dalsgaard J, Larson TP, Schou J, Thompson MJ, Ulrich R (2011) The torsional oscillation and the new solar cycle. J Phys Conf Ser 271:012074. doi:10.1088/1742-6596/ 271/1/012074

Howe R, Christensen-Dalsgaard J, Hill F, Komm R, Larson TP, Rempel M, Schou J, Thompson MJ (2013a) The high-latitude branch of the solar torsional oscillation in the rising phase of cycle 24 . Astrophys J Lett 767:L20. doi:10.1088/2041-8205/767/1/L20

Howe R, Christensen-Dalsgaard J, Hill F, Komm R, Larson TP, Schou J, Thompson MJ (2013b) The torsional oscillation and the timing of the solar cycle: is it maximum yet? In: Jain K, Tripathy SC, Hill F, Leibacher JW, Pevtsov AA (eds) Fifty years of seismology of the Sun and stars, ASP Conference Proceedings, vol 478. Astronomical Society of the Pacific, San Francisco, p 303

Huang RQ, Yu KN (1998) Stellar astrophysics. Springer, New York

Huber D, Ireland MJ, Bedding TR, Howell SB, Maestro V, Mérand A, Tuthill PG, White TR, Farrington CD, Goldfinger PJ, McAlister HA, Schaefer GH, Sturmann J, Sturmann L, ten Brummelaar TA, Turner NH (2012) Validation of the exoplanet Kepler-21b using PAVO/CHARA long-baseline interferometry. Mon Not R Astron Soc 423:L16-L20. doi:10.1111/j.1745-3933.2012.01242.x. arXiv:1202.5307

Huber D, Carter JA, Barbieri M, Miglio A, Deck KM, Fabrycky DC, Montet BT, Buchhave LA, Chaplin WJ, Hekker S, Montalbán J, Sanchis-Ojeda R, Basu S, Bedding TR, Campante TL, Christensen-Dalsgaard J, Elsworth YP, Stello D, Arentoft T, Ford EB, Gilliland RL, Handberg R, Howard AW, Isaacson H, Johnson JA, Karoff C, Kawaler SD, Kjeldsen H, Latham DW, Lund MN, Lundkvist M, Marcy GW, Metcalfe TS, Silva Aguirre V, Winn JN (2013) Stellar spin-orbit misalignment in a multiplanet system. Science 342:331-334. doi:10.1126/science.1242066. arXiv:1310.4503

Hummer DG, Mihalas D (1988) The equation of state for stellar envelopes. I. An occupation probability formalism for the truncation of internal partition functions. Astrophys J 331:794-814. doi:10.1086/ 166600

Iben I (1968) Solar neutrinos and the solar helium abundance. Phys Rev Lett 21:1208-1212. doi:10.1103/ PhysRevLett.21.1208

Iglesias CA, Rogers FJ (1996) Updated opal opacities. Astrophys J 464:943. doi:10.1086/177381

Irbah A, Meftah M, Hauchecorne A, Djafer D, Corbard T, Bocquier M, Momar Cisse E (2014) New space value of the solar oblateness obtained with PICARD. Astrophys J 785:89. doi:10.1088/0004-637X/ $785 / 2 / 89$

Jain K, Tripathy SC, Hill F (2009) Solar activity phases and intermediate-degree mode frequencies. Astrophys J 695:1567-1576. doi:10.1088/0004-637X/695/2/1567. arXiv:0902.1557

Jain K, Tripathy SC, Hill F, Leibacher JW, Pevtsov AA (eds) (2013) Fifty years of seismology of the sun and stars, ASP Conference Proceedings, vol 478. Astronomical Society of the Pacific, San Francisco

Jiménez-Reyes SJ, Régulo C, Pallé PL, Roca Cortés T (1998) Solar activity cycle frequency shifts of low-degree p-modes. Astron Astrophys 329:1119-1124

Judge PG, Burkepile J, de Toma G, Druckmüller M (2010) Historical eclipses and the recent solar minimum corona. In: Cranmer SR, Hoeksema JT, Kohl JL (eds) SOHO-23: understanding a peculiar solar minimum, ASP Conference Series, vol 428. Astronomical Society of the Pacific, San Francisco, p 171. arXiv: 1001.5278

Kippenhahn R, Weigert A, Weiss A (2012) Stellar structure and evolution. Astronomy and Astrophysics Library, Springer, Berlin. doi:10.1007/978-3-642-30304-3

Kjeldsen H, Bedding TR (1995) Amplitudes of stellar oscillations: the implications for asteroseismology. Astron Astrophys 293:87-106 arXiv:astro-ph/9403015

Kjeldsen H, Bedding TR, Baldry IK, Bruntt H, Butler RP, Fischer DA, Frandsen S, Gates EL, Grundahl F, Lang K, Marcy GW, Misch A, Vogt SS (2003) Confirmation of solar-like oscillations in $\eta$ bootis. Astron J 126:1483-1488. doi:10.1086/377315. arXiv:astro-ph/0305551

Kjeldsen H, Bedding TR, Butler RP, Christensen-Dalsgaard J, Kiss LL, McCarthy C, Marcy GW, Tinney CG, Wright JT (2005) Solar-like oscillations in $\alpha$ centauri B. Astrophys J 635:1281-1290. doi:10. 1086/497530. arXiv:astro-ph/0508609 
Kjeldsen H, Bedding TR, Christensen-Dalsgaard J (2008) Correcting stellar oscillation frequencies for near-surface effects. Astrophys J Lett 683:L175-L178. doi:10.1086/591667. arXiv:0807.1769

Koch DG, Borucki WJ, Basri G, Batalha NM, Brown TM, Caldwell D, Christensen-Dalsgaard J, Cochran WD, DeVore E, Dunham EW, Gautier TN III, Geary JC, Gilliland RL, Gould A, Jenkins J, Kondo Y, Latham DW, Lissauer JJ, Marcy G, Monet D, Sasselov D, Boss A, Brownlee D, Caldwell J, Dupree AK, Howell SB, Kjeldsen H, Meibom S, Morrison D, Owen T, Reitsema H, Tarter J, Bryson ST, Dotson JL, Gazis P, Haas MR, Kolodziejczak J, Rowe JF, Van Cleve JE, Allen C, Chandrasekaran H, Clarke BD, Li J, Quintana EV, Tenenbaum P, Twicken JD, Wu H (2010) Kepler mission design, realized photometric performance, and early science. Astrophys J Lett 713:L79. doi:10.1088/2041-8205/713/ 2/L79. arXiv: 1001.0268

Komm RW, Howard RF, Harvey JW (1993) Torsional oscillation patterns in photospheric magnetic features. Solar Phys 143:19-39. doi:10.1007/BF00619094

Korzennik SG, Rabello-Soares MC, Schou J, Larson TP (2013a) Accurate characterization of high-degree modes using MDI observations. Astrophys J 772:87. doi:10.1088/0004-637X/772/2/87

Korzennik SG, Rabello-Soares MC, Schou J, Larson TP (2013b) Accurate characterization of high-degree modes using MDI observations. Astrophys J 772:87. doi:10.1088/0004-637X/772/2/87

Kosovichev AG (1988a) An attempt to determine the structure of the solar core from observed G-mode frequencies. In: Christensen-Dalsgaard J, Frandsen S (eds) Advances in helio- and asteroseismology, IAU Symposium, vol 123. Reidel, Dordrecht, p 141

Kosovichev AG (1988b) Determination of the solar sound speed by an asymptotic inversion technique. In: Rolfe EJ (ed) Seismology of the Sun and Sun-like stars, ESA Special Publication, vol SP-286. ESA, Noordwijk, pp 533-537

Kosovichev AG (1990) Structure of the solar core inferred from inversion of frequencies of low-degree p-modes. In: Osaki Y, Shibahashi H (eds) Progress of seismology of the Sun and stars, Lecture Notes in Physics, vol 367. Springer, Berlin, p 319. doi:10.1007/3-540-53091-6_94

Kosovichev AG (1993) Seismic measurements of the helium abundance and the depth of stellar convection zones. Mon Not R Astron Soc 265:1053. doi:10.1093/mnras/265.4.1053

Kosovichev AG (1995) Determination of interior structure by inversion. In: Ulrich RK, Rhodes EJ Jr, Däppen W (eds) GONG '94: Helio- and astro-seismology from the Earth and space, ASP Conference Series, vol 76. Astronomical Society of the Pacific, San Francisco, p 89

Kosovichev AG (1996) Helioseismic measurements of elemental abundances in the sun's interior. Bull Astron Soc India 24:355

Kosovichev AG, Fedorova AV (1991) Construction of a seismic model of the Sun. Soviet Astron 35:507

Kosovichev AG, Schou J (1997) Detection of zonal shear flows beneath the Sun's surface from f-mode frequency splitting. Astrophys J Lett 482:L207-L210. doi:10.1086/310708

Kosovichev AG, Christensen-Dalsgaard J, Däppen W, Dziembowski WA, Gough DO, Thompson MJ (1992) Sources of uncertainty in direct seismological measurements of the solar helium abundance. Mon Not R Astron Soc 259:536-558. doi:10.1093/mnras/259.3.536

Krishna Swamy KS (1966) Profiles of strong lines in K-Dwarfs. Astrophys J 145:174. doi:10.1086/148752

Kuhn JR (1988a) Helioseismological splitting measurements and the nonspherical solar temperature structure. Astrophys J Lett 331:L131-L134. doi:10.1086/185251

Kuhn JR (1988b) Radial and temporal structure of the internal solar asphericity. In: Rolfe EJ (ed) Seismology of the Sun and Sun-like stars, ESA Special Publication, vol 286. ESA, Noordwijk, pp 87-90

Kuhn JR (1989) Calculating the internal solar asphericity from frequency splitting measurements. Solar Phys 123:1-5. doi:10.1007/BF00150007

Kuhn JR, Bush RI, Scherrer P, Scheick X (1998) The sun's shape and brightness. Nature 392:155. doi:10. $1038 / 32361$

Kumar P, Lu E (1991) The location of the source of high-frequency solar acoustic oscillations. Astrophys J Lett 375:L35-L39. doi:10.1086/186082

Kumar P, Franklin J, Goldreich P (1988) Distribution functions for the time-averaged energies of stochastically excited solar p-modes. Astrophys J 328:879-887. doi:10.1086/166345

Kumar P, Talon S, Zahn JP (1999) Angular momentum redistribution by waves in the Sun. Astrophys J 520:859-870. doi:10.1086/307464. arXiv:astro-ph/9902309

Kurucz RL (1991) New opacity calculations. In: Crivellari L, Hubeny I, Hummer DG (eds) Stellar atmospheres: beyond classical models, NATO ASI Series C, vol 341. Kluwer, Dordrecht, pp 441448. doi:10.1007/978-94-011-3554-2_42

Lamb H (1932) Hydrodynamics. Dover, New York 
Larson TP, Schou J (2008) Improvements in global mode analysis. J Phys Conf Ser 118:012083. doi:10. 1088/1742-6596/118/1/012083

Lazrek M, Baudin F, Bertello L, Boumier P, Charra J, Fierry-Fraillon D, Fossat E, Gabriel AH, García RA, Gelly B, Gouiffes C, Grec G, Pallé PL, Pérez Hernández F, Régulo C, Renaud C, Robillot JM, Roca Cortés T, Turck-Chièze S, Ulrich RK (1997) First results on p modes from GOLF experiment. Solar Phys 175:227-246. doi:10.1023/A:1004942929956

Ledoux P (1949) Contributions à l'Etude de la Structure Interne des Etoiles et de leur Stabilité. Memoires of the Societe Royale des Sciences de Liege 9:3-294

Ledoux P, Walraven T (1958) Variable stars. Handbuch der Physik 51:353-604. doi:10.1007/ 978-3-642-45908-5_6

Lefebvre S, Kosovichev AG, Rozelot JP (2007) Helioseismic test of nonhomologous solar radius changes with the 11 year activity cycle. Astrophys J Lett 658:L135-L138. doi:10.1086/515394. arXiv:astro-ph/0702497

Leibacher JW, Stein RF (1971) A new description of the solar five-minute oscillation. Astrophys Lett Comm 7:191-192

Leighton RB, Noyes RW, Simon GW (1962) Velocity fields in the solar atmosphere. I. Preliminary report. Astrophys J 135:474. doi:10.1086/147285

Li LH, Robinson FJ, Demarque P, Sofia S, Guenther DB (2002) Inclusion of turbulence in solar modeling. Astrophys J 567:1192-1201. doi:10.1086/338352. arXiv:astro-ph/0109078

Li LH, Basu S, Sofia S, Robinson FJ, Demarque P, Guenther DB (2003) Global parameter and helioseismic tests of solar variability models. Astrophys J 591:1267-1284. doi:10.1086/375484

Libbrecht KG (1989) Solar p-mode frequency splittings. Astrophys J 336:1092-1097. doi:10.1086/167079

Libbrecht KG, Woodard MF (1990) Solar-cycle effects on solar oscillation frequencies. Nature 345:779_ 782. doi: $10.1038 / 345779 \mathrm{a} 0$

Libbrecht KG, Woodard MF, Kaufman JM (1990) Frequencies of solar oscillations. Astrophys J Suppl Ser 74:1129-1149. doi:10.1086/191528

Lo L, Hoeksema JT, Scherrer PH (2010) Three cycles of the solar toroidal magnetic field and this peculiar minimum. In: Cranmer SR, Hoeksema JT, Kohl JL (eds) SOHO-23: understanding a peculiar solar minimum, ASP Conference Series, vol 428. Astronomical Society of the Pacific, San Francisco, p 109. arXiv:1003.3981

Lodders K (2010) Solar system abundances of the elements. In: Goswami A, Reddy BE (eds) Principles and perspectives in cosmochemistry. Springer, Berlin, p 379. doi:10.1007/978-3-642-10352-0_8. arXiv: 1010.2746

Ludwig HG, Freytag B, Steffen M (1999) A calibration of the mixing-length for solar-type stars based on hydrodynamical simulations. I. Methodical aspects and results for solar metallicity. Astron Astrophys 346:111-124. arXiv:astro-ph/9811179

Lund MN, Miesch MS, Christensen-Dalsgaard J (2014) Differential rotation in main-sequence solar-like stars: qualitative inference from asteroseismic data. Astrophys J 790:121. doi:10.1088/0004-637X/ 790/2/121. arXiv:1406.7873

Maeder A (2009) Physics, Formation and Evolution of Rotating Stars. Astronomy and Astrophysics Library, Springer, Berlin. doi:10.1007/978-3-540-76949-1

Magic Z, Collet R, Asplund M, Trampedach R, Hayek W, Chiavassa A, Stein RF, Nordlund Å (2013) The stagger-grid: a grid of 3D stellar atmosphere models. I. Methods and general properties. Astron Astrophys 557:A26. doi:10.1051/0004-6361/201321274. arXiv:1302.2621

Mamajek EE, Prsa A, Torres G, Harmanec P, Asplund M, Bennett PD, Capitaine N, Christensen-Dalsgaard J, Depagne E, Folkner WM, Haberreiter M, Hekker S, Hilton JL, Kostov V, Kurtz DW, Laskar J, Mason BD, Milone EF, Montgomery MM, Richards MT, Schou J, Stewart SG (2015) IAU 2015 resolution B3 on recommended nominal conversion constants for selected solar and planetary properties. ArXiv e-prints https://www.iau.org/administration/resolutions/general_assemblies/. arXiv:1510.07674

Manoharan PK (2012) Three-dimensional evolution of solar wind during solar cycles 22-24. Astrophys J 751:128. doi:10.1088/0004-637X/751/2/128. arXiv:1203.6715

Mathur S, Metcalfe TS, Woitaszek M, Bruntt H, Verner GA, Christensen-Dalsgaard J, Creevey OL, Doğan G, Basu S, Karoff C, Stello D, Appourchaux T, Campante TL, Chaplin WJ, García RA, Bedding TR, Benomar O, Bonanno A, Deheuvels S, Elsworth Y, Gaulme P, Guzik JA, Handberg R, Hekker S, Herzberg W, Monteiro MJPFG, Piau L, Quirion PO, Régulo C, Roth M, Salabert D, Serenelli A, Thompson MJ, Trampedach R, White TR, Ballot J, Brandão IM, Molenda-Zakowicz J, Kjeldsen 
H, Twicken JD, Uddin K, Wohler B (2012) A uniform asteroseismic analysis of 22 solar-type stars observed by Kepler. Astrophys J 749:152. doi:10.1088/0004-637X/749/2/152. arXiv:1202.2844

Mazumdar A (2005) Asteroseismic diagrams for solar-type stars. Astron Astrophys 441:1079-1086. doi:10. 1051/0004-6361:20042348. arXiv:astro-ph/0507077

Mazumdar A, Roxburgh I (2003) The asteroseismic diagram for $\ell=0,1$ p-modes. In: Thompson MJ, Cunha MS, Monteiro MJPFG (eds) Asteroseismology across the HR diagram. Kluwer, Dordrecht, pp 477-480

Mazumdar A, Monteiro MJPFG, Ballot J, Antia HM, Basu S, Houdek G, Mathur S, Cunha MS, Silva Aguirre V, García RA, Salabert D, Verner GA, Christensen-Dalsgaard J, Metcalfe TS, Chaplin WJ (2012) Acoustic glitches in solar-type stars from Kepler. Astron Nachr 333:1040-1043. doi:10.1002/ asna.201211825

Mazumdar A, Monteiro MJPFG, Ballot J, Antia HM, Basu S, Houdek G, Mathur S, Cunha MS, Silva Aguirre V, García RA, Salabert D, Verner GA, Christensen-Dalsgaard J, Metcalfe TS, Sanderfer DT, Seader SE, Smith JC, Chaplin WJ (2014) Measurement of acoustic glitches in solar-type stars from oscillation frequencies observed by Kepler. Astrophys J 782:18. doi:10.1088/0004-637X/782/1/18. arXiv:1312.4907

Meftah M, Irbah A, Hauchecorne A, Corbard T, Turck-Chièze S, Hochedez JF, Boumier P, Chevalier A, Dewitte S, Mekaoui S, Salabert D (2015) On the determination and constancy of the solar oblateness. Solar Phys 290:673-687. doi:10.1007/s11207-015-0655-6

Mendoza C, Seaton MJ, Buerger P, Bellorín A, Meléndez M, González J, Rodríguez LS, Delahaye F, Palacios E, Pradhan AK, Zeippen CJ (2007) OPserver: interactive online computations of opacities and radiative accelerations. Mon Not R Astron Soc 378:1031-1035. doi:10.1111/j.1365-2966.2007. 11837.x. arXiv:0704.1583

Metcalfe TS, Monteiro MJPFG, Thompson MJ, Molenda-Zakowicz J, Appourchaux T, Chaplin WJ, Doğan G, Eggenberger P, Bedding TR, Bruntt H, Creevey OL, Quirion PO, Stello D, Bonanno A, Silva Aguirre V, Basu S, Esch L, Gai N, Di Mauro MP, Kosovichev AG, Kitiashvili IN, Suárez JC, Moya A, Piau L, García RA, Marques JP, Frasca A, Biazzo K, Sousa SG, Dreizler S, Bazot M, Karoff C, Frandsen S, Wilson PA, Brown TM, Christensen-Dalsgaard J, Gilliland RL, Kjeldsen H, Campante TL, Fletcher ST, Handberg R, Régulo C, Salabert D, Schou J, Verner GA, Ballot J, Broomhall AM, Elsworth Y, Hekker S, Huber D, Mathur S, New R, Roxburgh IW, Sato KH, White TR, Borucki WJ, Koch DG, Jenkins JM (2010) A precise asteroseismic age and radius for the evolved sun-like star KIC 11026764. Astrophys J 723:1583-1598. doi:10.1088/0004-637X/723/2/1583. arXiv:1010.4329

Metcalfe TS, Chaplin WJ, Appourchaux T, García RA, Basu S, Brandão I, Creevey OL, Deheuvels S, Doğan G, Eggenberger P, Karoff C, Miglio A, Stello D, Yıldız M, Çelik Z, Antia HM, Benomar O, Howe R, Régulo C, Salabert D, Stahn T, Bedding TR, Davies GR, Elsworth Y, Gizon L, Hekker S, Mathur S, Mosser B, Bryson ST, Still MD, Christensen-Dalsgaard J, Gilliland RL, Kawaler SD, Kjeldsen H, Ibrahim KA, Klaus TC, Li J (2012) Asteroseismology of the solar analogs 16 Cyg A and B from Kepler observations. Astrophys J Lett 748:L10. doi:10.1088/2041-8205/748/1/L10. arXiv:1201.5966

Metcalfe TS, Creevey OL, Dogan G, Mathur S, Xu H, Bedding TR, Chaplin WJ, Christensen-Dalsgaard J, Karoff C, Trampedach R, Benomar O, Brown BP, Buzasi DL, Campante TL, Celik Z, Cunha MS, Davies GR, Deheuvels S, Derekas A, Di Mauro MP, Garcia RA, Guzik JA, Howe R, MacGregor KB, Mazumdar A, Montalban J, Monteiro MJPFG, Salabert D, Serenelli A, Stello D, Steslicki M, Suran MD, Yildiz M, Aksoy C, Elsworth Y, Gruberbauer M, Guenther DB, Lebreton Y, Molaverdikhani K, Pricopi D, Simoniello R, White TR (2014) Properties of 42 solar-type Kepler targets from the asteroseismic modeling portal. Astrophys J Suppl Ser 214:27. doi:10.1088/0067-0049/214/2/27. arXiv: 1402.3614

Mihalas D, Däppen W, Hummer DG (1988) The equation of state for stellar envelopes. II. Algorithm and selected results. Astrophys J 331:815-825. doi:10.1086/166601

Monteiro MJPFG, Thompson MJ (1998) Looking for variations with latitude of the base of the solar convection zone. In: Korzennik S, Wilson A (eds) Structure and dynamics of the interior of the Sun and Sun-like stars, ESA Special Publication, vol SP-418. ESA, Noordwijk, p 819

Monteiro MJPFG, Christensen-Dalsgaard J, Thompson MJ (1994) Seismic study of overshoot at the base of the solar convective envelope. Astron Astrophys 283:247-262

Mosser B, Goupil MJ, Belkacem K, Marques JP, Beck PG, Bloemen S, De Ridder J, Barban C, Deheuvels S, Elsworth Y, Hekker S, Kallinger T, Ouazzani RM, Pinsonneault M, Samadi R, Stello D, García RA, Klaus TC, Li J, Mathur S, Morris RL (2012) Spin down of the core rotation in red giants. Astron Astrophys 548:A10. doi:10.1051/0004-6361/201220106. arXiv:1209.3336 
Mursula K, Zieger B, Vilppola JH (2003) Mid-term quasi-periodicities in geomagnetic activity during the last 15 solar cycles: Connection to solar dynamo strength To the memory of Karolen I. Paularena (1957-2001). Solar Phys 212:201-207. doi:10.1023/A:1022980029618

Naur P (1954) Two models for the interior of the Sun. Astrophys J 119:365. doi:10.1086/145834

Neuforge-Verheecke C, Goriely S, Guzik JA, Swenson FJ, Bradley PA (2001a) Seismological tests of solar models calculated with the NACRE reaction rates and the Grevesse and Sauval 1998 mixture. Astrophys J 550:493-502. doi:10.1086/319708

Neuforge-Verheecke C, Guzik JA, Keady JJ, Magee NH, Bradley PA, Noels A (2001b) Helioseismic tests of the new Los Alamos LEDCOP opacities. Astrophys J 561:450-454. doi:10.1086/323229

Nishizawa Y, Shibahashi H (1995) Implication of long-term frequency variation. In: Ulrich RK, Rhodes EJ Jr, Däppen W (eds) GONG '94: Helio- and astro-seismology from the Earth and space, ASP Conference Series, vol 76. Astronomical Society of the Pacific, San Francisco, p 280

Ogden Abell G (1955) A model for the internal structure of the Sun. Astrophys J 121:430. doi:10.1086/ 146004

Otí Floranes H, Christensen-Dalsgaard J, Thompson MJ (2005) The use of frequency-separation ratios for asteroseismology. Mon Not R Astron Soc 356:671-679. doi:10.1111/j.1365-2966.2004.08487.x

Pallé PL, Régulo C, Roca Cortés T (1989) Solar cycle induced variations of the low L solar acoustic spectrum. Astron Astrophys 224:253-258

Pérez Hernández F, Christensen-Dalsgaard J (1994a) The phase function for stellar acoustic oscillations-II. Effects of filtering. Mon Not R Astron Soc 267:111

Pérez Hernández F, Christensen-Dalsgaard J (1994b) The phase function for stellar acoustic oscillations-III. The solar case. Mon Not R Astron Soc 269:475

Pesnell WD, Thompson BJ, Chamberlin PC (2012) The solar dynamics observatory (SDO). Solar Phys 275:3-15. doi:10.1007/s11207-011-9841-3

Piau L, Collet R, Stein RF, Trampedach R, Morel P, Turck-Chièze S (2014) Models of solar surface dynamics: impact on eigenfrequencies and radius. Mon Not R Astron Soc 437:164-175. doi:10.1093/ mnras/stt1866. arXiv:1309.7179

Pijpers FP (1997) Solar rotation inversions and the relationship between a-coefficients and mode splittings. Astron Astrophys 326:1235-1240. arXiv:astro-ph/9705234

Pijpers FP (1998) Helioseismic determination of the solar gravitational quadrupole moment. Mon Not R Astron Soc 297:L76-L80. doi:10.1046/j.1365-8711.1998.01801.x. arXiv:astro-ph/9804258

Pijpers FP, Thompson MJ (1992) Faster formulations of the optimally localized averages method for helioseismic inversions. Astron Astrophys 262:L33-L36

Pinsonneault MH, Kawaler SD, Sofia S, Demarque P (1989) Evolutionary models of the rotating sun. Astrophys J 338:424-452. doi:10.1086/167210

Pinsonneault MH, Elsworth Y, Epstein C, Hekker S, Mészáros S, Chaplin WJ, Johnson JA, García RA, Holtzman J, Mathur S, García Pérez A, Silva Aguirre V, Girardi L, Basu S, Shetrone M, Stello D, Allende Prieto C, An D, Beck P, Beers TC, Bizyaev D, Bloemen S, Bovy J, Cunha K, De Ridder J, Frinchaboy PM, García-Hernández DA, Gilliland R, Harding P, Hearty FR, Huber D, Ivans I, Kallinger T, Majewski SR, Metcalfe TS, Miglio A, Mosser B, Muna D, Nidever DL, Schneider DP, Serenelli A, Smith VV, Tayar J, Zamora O, Zasowski G (2014) The APOKASC catalog: an asteroseismic and spectroscopic joint survey of targets in the Kepler fields. Astrophys J Suppl Ser 215:19. doi:10.1088/ 0067-0049/215/2/19. arXiv:1410.2503

Pochoda P, Reeves H (1964) A revised solar model with a solar neutrino spectrum. Planetary Sp Sci 12:119126. doi:10.1016/0032-0633(64)90116-3

Pottasch EM, Butcher HR, van Hoesel FHJ (1992) Solar-like oscillations on $\alpha$ Centauri A. Astron Astrophys 264:138-146

Prandtl L (1925) Bericht über Untersuchungen zur ausgebildeten Turbulenz. Z Angew Math Mech 5:136139

Proffitt CR, Michaud G (1991) Gravitational settling in solar models. Astrophys J 380:238-250. doi:10. $1086 / 170580$

Quirion PO, Christensen-Dalsgaard J, Arentoft T (2010) Automatic determination of stellar parameters via asteroseismology of stochastically oscillating stars: comparison with direct measurements. Astrophys J 725:2176-2189. doi:10.1088/0004-637X/725/2/2176. arXiv:1009.5131

Rabello-Soares MC (2012) Solar-cycle variation of sound speed near the solar surface. Astrophys J 745:184. doi:10.1088/0004-637X/745/2/184. arXiv:1110.5596 
Rabello-Soares MC, Basu S, Christensen-Dalsgaard J (1999) On the choice of parameters in solarstructure inversion. Mon Not R Astron Soc 309:35-47. doi:10.1046/j.1365-8711.1999.02785.x. arXiv:astro-ph/9905107

Rabello-Soares MC, Basu S, Christensen-Dalsgaard J, Di Mauro MP (2000) The potential of solar highdegree modes for structure inversion. Solar Phys 193:345-356. doi:10.1023/A:1005228518674

Rabello-Soares MC, Korzennik SG, Schou J (2006) High-degree mode frequencies: changes with solar cycle. In: SOHO 18/Gong 2006/HELAS I: beyond the spherical Sun, ESA Special Publication, vol SP-624. ESA, Noordwijk

Rabello-Soares MC, Korzennik SG, Schou J (2008) Variations of the solar acoustic high-degree mode frequencies over solar cycle 23. Adv Space Res 41:861-867. doi:10.1016/j.asr.2007.03.014. arXiv:0712.3608

Régulo C, Jimenez A, Pallé PL, Pérez Hernández F, Roca Cortés T (1994) Variation of the frequencies of very low $l$ p-modes. Astrophys J 434:384-388. doi:10.1086/174737

Reiter J, Kosovichev AG, Rhodes EJ Jr, Schou J (2003) Accurate measurements of SOI/MDI high-degree frequencies and frequency splittings. In: Sawaya-Lacoste H (ed) Local and global helioseismology: the present and future, ESA Special Publication, vol SP-517. ESA, Noordwijk, pp 369-372

Rempel M (2004) Overshoot at the base of the solar convection zone: a semianalytical approach. Astrophys J 607:1046-1064. doi:10.1086/383605

Rhodes EJ, Reiter J, Schou J, Larson T, Scherrer P, Brooks J, McFaddin P, Miller B, Rodriguez J, Yoo J (2011) Temporal changes in the frequencies of the solar p-mode oscillations during solar cycle 23. In: Prasad Choudhary D, Strassmeier KG (eds) The physics of Sun and star spots, IAU Symposium, vol 273. Cambridge University Press, Cambridge, pp 389-393. doi:10.1017/S1743921311015614

Rhodes EJ Jr, Ulrich RK, Simon GW (1977) Observations of nonradial p-mode oscillations on the sun. Astrophys J 218:901-919. doi:10.1086/155745

Rhodes EJ Jr, Reiter J, Schou J (2003) High-degree p-modes and the Sun's evolving surface. In: SawayaLacoste H (ed) Local and global helioseismology: the present and future, ESA Special Publication, vol SP-517. ESA, Noordwijk, pp 173-182

Richard O, Vauclair S (1997) Local mixing near the solar core, neutrino fluxes and helioseismology. Astron Astrophys 322:671-673. arXiv:astro-ph/9609133

Richard O, Vauclair S, Charbonnel C, Dziembowski WA (1996) New solar models including helioseismological constraints and light-element depletion. Astron Astrophys 312:1000-1011. arXiv:astro-ph/9601136

Richard O, Dziembowski WA, Sienkiewicz R, Goode PR (1998) On the accuracy of helioseismic determination of solar helium abundance. Astron Astrophys 338:756-760

Rimmele TR, Goode PR, Harold E, Stebbins RT (1995) Dark lanes in granulation and the excitation of solar oscillations. Astrophys J Lett 444:L119-L122. doi:10.1086/187874

Ritzwoller MH, Lavely EM (1991) A unified approach to the helioseismic forward and inverse problems of differential rotation. Astrophys J 369:557-566. doi:10.1086/169785

Robinson FJ, Demarque P, Li LH, Sofia S, Kim YC, Chan KL, Guenther DB (2004) Three-dimensional simulations of the upper radiation-convection transition layer in subgiant stars. Mon Not R Astron Soc 347:1208-1216. doi:10.1111/j.1365-2966.2004.07296.x

Roeder RC, Demarque PR (1966) Solar evolution and Brans-Dicke cosmology. Astrophys J 144:1016. doi:10.1086/148700

Rogers FJ, Iglesias CA (1992) Radiative atomic Rosseland mean opacity tables. Astrophys J Suppl Ser 79:507-568. doi:10.1086/191659

Rogers FJ, Nayfonov A (2002) Updated and expanded OPAL equation-of-state tables: implications for helioseismology. Astrophys J 576:1064-1074. doi:10.1086/341894

Rogers FJ, Swenson FJ, Iglesias CA (1996) OPAL equation-of-state tables for astrophysical applications. Astrophys J 456:902. doi:10.1086/176705

Rogers TM, Glatzmaier GA, Jones CA (2006) Numerical simulations of penetration and overshoot in the Sun. Astrophys J 653:765-773. doi:10.1086/508482. arXiv:astro-ph/0601668

Rosenthal CS, Christensen-Dalsgaard J, Houdek G, Monteiro MJPFG, Nordlund Å, Trampedach R (1995) Seismology of the solar surface regions. In: Hoeksema JT, Domingo V, Fleck B, Battrick B (eds) Helioseismology, ESA Special Publication, vol SP-376. ESA, Noordwijk, pp 459-464

Rosenthal CS, Christensen-Dalsgaard J, Kosovichev AG, Nordlund Å, Reiter J, Rhodes EJ Jr, Schou J, Stein RF, Trampedach R (1998) Tests of convective frequency effects with SOI/MDI high-degree data. In: 
Korzennik S (ed) Structure and dynamics of the interior of the Sun and Sun-like stars, ESA Special Publication, vol 418. ESA, Noordwijk, p 521. arXiv:astro-ph/9807066

Rosenthal CS, Christensen-Dalsgaard J, Nordlund Å, Stein RF, Trampedach R (1999) Convective contributions to the frequencies of solar oscillations. Astron Astrophys 351:689-700. arXiv:astro-ph/9803206

Roxburgh IW (1985) Instabilities, mixing and solar neutrinos. In: Cherry ML, Lande K, Fowler WA (eds) Solar neutrinos and neutrino astronomy, AIP Conference Series, vol 126. American Institute of Physics, New York, pp 88-92. doi:10.1063/1.35166

Roxburgh IW (2014) Surface layer independent model fitting by phase matching: theory and application to HD49933 an HD177153 (aka Perky). Astron Astrophys 574:A45. doi:10.1051/0004-6361/ 201425289. arXiv:1406.6491

Roxburgh IW, Vorontsov SV (1993) Asymptotic theory of low-degree stellar acoustic oscillations. In: Weiss WW, Baglin A (eds) Inside the stars: IAU Colloquium 137, ASP Conference Series, vol 40. Astronomical Society of the Pacific, San Francisco, p 535

Roxburgh IW, Vorontsov SV (1994) Seismology of the solar envelope: the base of the convective zone as seen in the phase shift of acoustic waves. Mon Not R Astron Soc 268:880. doi:10.1093/mnras/268.4. 880

Roxburgh IW, Vorontsov SV (2003) The ratio of small to large separations of acoustic oscillations as a diagnostic of the interior of solar-like stars. Astron Astrophys 411:215-220. doi:10.1051/0004-6361: 20031318

Roxburgh IW, Vorontsov SV (2013) On the use of the ratio of small to large separations in asteroseismic model fitting. Astron Astrophys 560:A2. doi:10.1051/0004-6361/201321333. arXiv:1307.5069

Sackmann IJ, Boothroyd AI (2003) Our Sun. V. A bright young Sun consistent with helioseismology and warm temperatures on ancient Earth and Mars. Astrophys J 583:1024-1039. doi:10.1086/345408. arXiv:astro-ph/0210128

Saio H (1992) Opacity in the solar radiative interior inferred from 5-min oscillations. Mon Not R Astron Soc 258:491-496. doi:10.1093/mnras/258.3.491

Salabert D, Fossat E, Gelly B, Kholikov S, Grec G, Lazrek M, Schmider FX (2004) Solar p modes in 10 years of the IRIS network. Astron Astrophys 413:1135-1142. doi:10.1051/0004-6361:20031541

Salpeter EE (1969) Equations of state and stellar evolution. In: Kumar SS (ed) Low-luminosity stars. Gordon and Breach, New York, p 205

Samadi R, Goupil MJ (2001) Excitation of stellar p-modes by turbulent convection. I. Theoretical formulation. Astron Astrophys 370:136-146. doi:10.1051/0004-6361:20010212. arXiv:astro-ph/0101109

Samadi R, Nordlund Å, Stein RF, Goupil MJ, Roxburgh I (2003a) Numerical 3D constraints on convective eddy time-correlations: consequences for stochastic excitation of solar p modes. Astron Astrophys 404:1129-1137. doi:10.1051/0004-6361:20030504. arXiv:astro-ph/0304457

Samadi R, Nordlund Å, Stein RF, Goupil MJ, Roxburgh I (2003b) Numerical constraints on the model of stochastic excitation of solar-type oscillations. Astron Astrophys 403:303-312. doi:10.1051/0004-6361: 20030356. arXiv:astro-ph/0303198

Samadi R, Georgobiani D, Trampedach R, Goupil MJ, Stein RF, Nordlund Å (2007) Excitation of solarlike oscillations across the HR diagram. Astron Astrophys 463:297-308. doi:10.1051/0004-6361: 20041953. arXiv:astro-ph/0611762

Schatzman E (1985) Mixing versus turbulent diffusion mixing and the solar neutrino problem. In: Cherry ML, Lande K, Fowler WA (eds) Solar neutrinos and neutrino astronomy, AIP Conference Series, vol 126. American Institute of Physics, New York, pp 69-74. doi:10.1063/1.35164

Scherrer PH, Bogart RS, Bush RI, Hoeksema JT, Milford P, Schou J, Pope T, Rosenberg W, Springer L, Tarbell T, Title A, Wolfson J, Zayer I (1995) Status of the solar oscillations investigation: Michelson Doppler Imager. In: Ulrich RK, Rhodes EJ Jr, Däppen W (eds) GONG '94: Helio- and astro-seismology from the Earth and Space, ASP Conference Series, vol 76. Astronomical Society of the Pacific, San Francisco, p 402

Scherrer PH, Schou J, Bush RI, Kosovichev AG, Bogart RS, Hoeksema JT, Liu Y, Duvall TL, Zhao J, Title AM, Schrijver CJ, Tarbell TD, Tomczyk S (2012) The helioseismic and magnetic imager (HMI) investigation for the solar dynamics observatory (SDO). Solar Phys 275:207-227. doi:10. 1007/s11207-011-9834-2

Schlattl H, Bonanno A, Paternò L (1999) Signatures of the efficiency of solar nuclear reactions in the neutrino experiments. Phys Rev D 60:113002. doi:10.1103/PhysRevD.60.113002. arXiv:astro-ph/9902354

Schmitt JR, Basu S (2015) Modeling the asteroseismic surface term across the HR diagram. Astrophys J 808:123. doi:10.1088/0004-637X/808/2/123. arXiv:1506.06678 
Schou J (1999) Migration of zonal flows detected using Michelson Doppler imager F-mode frequency splittings. Astrophys J Lett 523:L181-L184. doi:10.1086/312279

Schou J, Buzasi DL (2001) Observations of p-modes in $\alpha$ Cen. In: Wilson A, Pallé PL (eds) SOHO 10/GONG 2000 Workshop: Helio- and asteroseismology at the dawn of the millennium, ESA Special Publication, vol SP-464. ESA, Noordwijk, pp 391-394

Schou J, Kosovichev AG, Goode PR, Dziembowski WA (1997) Determination of the Sun's seismic radius from the SOHO Michelson Doppler imager. Astrophys J Lett 489:L197. doi:10.1086/316782

Schou J, Antia HM, Basu S, Bogart RS, Bush RI, Chitre SM, Christensen-Dalsgaard J, di Mauro MP, Dziembowski WA, Eff-Darwich A, Gough DO, Haber DA, Hoeksema JT, Howe R, Korzennik SG, Kosovichev AG, Larsen RM, Pijpers FP, Scherrer PH, Sekii T, Tarbell TD, Title AM, Thompson MJ, Toomre J (1998a) Helioseismic studies of differential rotation in the solar envelope by the solar oscillations investigation using the Michelson Doppler imager. Astrophys J 505:390-417. doi:10. $1086 / 306146$

Schou J, Christensen-Dalsgaard J, Howe R, Larsen RM, Thompson MJ, Toomre J (1998b) Slow poles and shearing flows from heliospheric observations with MDI and GONG spanning a year. In: Korzennik $\mathrm{S}$ (ed) Structure and dynamics of the interior of the Sun and Sun-like stars, ESA Special Publication, vol 418. ESA, Noordwijk, p 845

Schou J, Scherrer PH, Bush RI, Wachter R, Couvidat S, Rabello-Soares MC, Bogart RS, Hoeksema JT, Liu Y, Duvall TL, Akin DJ, Allard BA, Miles JW, Rairden R, Shine RA, Tarbell TD, Title AM, Wolfson CJ, Elmore DF, Norton AA, Tomczyk S (2012) Design and ground calibration of the helioseismic and magnetic imager (HMI) instrument on the solar dynamics observatory (SDO). Solar Phys 275:229259. doi:10.1007/s11207-011-9842-2

Schunker H, Schou J, Ball WH (2016a) Asteroseismic inversions for radial differential rotation of Sun-like stars: sensitivity to uncertainties. Astron Astrophys 586:A24. doi:10.1051/0004-6361/201525937. arXiv: 1511.08365

Schunker H, Schou J, Ball WH, Nielsen MB, Gizon L (2016b) Asteroseismic inversions for radial differential rotation of Sun-like stars: ensemble fits. Astron Astrophys 586:A79. doi:10.1051/0004-6361/ 201527485. arXiv:1512.07169

Schwarzschild M (1946) On the helium content of the Sun. Astrophys J 104:203. doi:10.1086/144846

Schwarzschild M, Howard R, Härm R (1957) Inhomogeneous stellar models. V. A solar model with convective envelope and inhomogeneous interior. Astrophys J 125:233. doi:10.1086/146297

Sears RL (1964) Helium content and neutrino fluxes in solar models. Astrophys J 140:477. doi:10.1086/ 147942

Sekii T (1997) Internal solar rotation. In: Provost J, Schmider FX (eds) Sounding solar and stellar interiors, IAU Symposium, vol 181. Kluwer, Dordrecht, pp 189-202

Sekii T, Shibahashi H (1989) An asymptotic inversion method of inferring the sound velocity distribution in the sun from the spectrum of p-mode oscillations. Publ Astron Soc Japan 41:311-331

Serebryanskiy A, Chou DY (2005) Comparison of solar cycle variations of solar p-mode frequencies from GONG and MDI. Astrophys J 633:1187-1190. doi:10.1086/491467

Serenelli AM, Basu S (2010) Determining the initial helium abundance of the Sun. Astrophys J 719:865872. doi:10.1088/0004-637X/719/1/865. arXiv:1006.0244

Serenelli AM, Basu S, Ferguson JW, Asplund M (2009) New solar composition: the problem with solar models revisited. Astrophys J Lett 705:L123-L127. doi:10.1088/0004-637X/705/2/L123. arXiv:0909.2668

Sheeley NR Jr (2010) What's so peculiar about the Cycle 23/24 solar minimum? In: Cranmer SR, Hoeksema JT, Kohl JL (eds) SOHO-23: understanding a peculiar solar minimum, ASP Conference Series, vol 428. Astronomical Society of the Pacific, San Francisco, p 3. arXiv:1005.3834

Shibahashi H (1988) Inverse problem: acoustic potential vs acoustic length. In: Christensen-Dalsgaard J, Frandsen S (eds) Advances in helio- and asteroseismology, IAU Symposium, vol 123. Reidel, Dordrecht, p 133

Shibahashi H, Takata M (1996) A seismic solar model deduced from the sound-speed distribution and an estimate of the neutrino fluxes. Publ Astron Soc Japan 48:377-387. doi:10.1093/pasj/48.2.377

Shibahashi H, Hiremath KM, Takata M (1998) Seismic model of the solar convective envelope. In: Korzennik $\mathrm{S}$ (ed) Structure and dynamics of the interior of the Sun and Sun-like stars, ESA Special Publication, vol 418. ESA, Noordwijk, p 537

Silva Aguirre V, Casagrande L, Basu S, Campante TL, Chaplin WJ, Huber D, Miglio A, Serenelli AM, Ballot J, Bedding TR, Christensen-Dalsgaard J, Creevey OL, Elsworth Y, García RA, Gilliland RL, Hekker S, 
Kjeldsen H, Mathur S, Metcalfe TS, Monteiro MJPFG, Mosser B, Pinsonneault MH, Stello D, Weiss A, Tenenbaum P, Twicken JD, Uddin K (2012) Verifying asteroseismically determined parameters of Kepler stars using hipparcos parallaxes: self-consistent stellar properties and distances. Astrophys J 757:99. doi:10.1088/0004-637X/757/1/99. arXiv:1208.6294

Silva Aguirre V, Basu S, Brandão IM, Christensen-Dalsgaard J, Deheuvels S, Doğan G, Metcalfe TS, Serenelli AM, Ballot J, Chaplin WJ, Cunha MS, Weiss A, Appourchaux T, Casagrande L, Cassisi S, Creevey OL, García RA, Lebreton Y, Noels A, Sousa SG, Stello D, White TR, Kawaler SD, Kjeldsen $\mathrm{H}$ (2013) Stellar ages and convective cores in field main-sequence stars: first asteroseismic application to two Kepler targets. Astrophys J 769:141. doi:10.1088/0004-637X/769/2/141. arXiv:1304.2772

Silva Aguirre V, Davies GR, Basu S, Christensen-Dalsgaard J, Creevey O, Metcalfe TS, Bedding TR, Casagrande L, Handberg R, Lund MN, Nissen PE, Chaplin WJ, Huber D, Serenelli AM, Stello D, Van Eylen V, Campante TL, Elsworth Y, Gilliland RL, Hekker S, Karoff C, Kawaler SD, Kjeldsen H, Lundkvist MS (2015) Ages and fundamental properties of Kepler exoplanet host stars from asteroseismology. Mon Not R Astron Soc 452:2127-2148. doi:10.1093/mnras/stv1388. arXiv:1504.07992

Simoniello R, Jain K, Tripathy SC, Turck-Chièze S, Baldner C, Finsterle W, Hill F, Roth M (2013) The quasi-biennial periodicity as a window on the solar magnetic dynamo configuration. Astrophys $\mathrm{J}$ 765:100. doi:10.1088/0004-637X/765/2/100. arXiv:1210.6796

Snodgrass HB (1983) Magnetic rotation of the solar photosphere. Astrophys J 270:288-299. doi:10.1086/ 161121

Snodgrass HB (1984) Separation of large-scale photospheric Doppler patterns. Solar Phys 94:13-31. doi:10. 1007/BF00154804

Snodgrass HB (1992) Synoptic observations of large scale velocity patterns on the Sun. In: Harvey KL (ed) The solar cycle, ASP Conference Series, vol 27. Astronomical Society of the Pacific, San Francisco, p 205

Snodgrass HB, Ulrich RK (1990) Rotation of Doppler features in the solar photosphere. Astrophys J 351:309-316. doi:10.1086/168467

Sofia S, Basu S, Demarque P, Li L, Thuillier G (2005) The nonhomologous nature of solar diameter variations. Astrophys J Lett 632:L147-L150. doi:10.1086/498068. arXiv:astro-ph/0509816

Stein R, Georgobiani D, Trampedach R, Ludwig HG, Nordlund A (2004) Excitation of radial P-modes in the Sun and stars. Solar Phys 220:229-242. doi:10.1023/B:SOLA.0000031458.35121.33

Stein RF, Nordlund Å (2001) Solar oscillations and convection. II. Excitation of radial oscillations. Astrophys J 546:585-603. doi:10.1086/318218. arXiv:astro-ph/0008048

Stello D, Chaplin WJ, Bruntt H, Creevey OL, García-Hernández A, Monteiro MJPFG, Moya A, Quirion PO, Sousa SG, Suárez JC, Appourchaux T, Arentoft T, Ballot J, Bedding TR, Christensen-Dalsgaard J, Elsworth Y, Fletcher ST, García RA, Houdek G, Jiménez-Reyes SJ, Kjeldsen H, New R, Régulo C, Salabert D, Toutain T (2009) Radius determination of solar-type stars using asteroseismology: what to expect from the Kepler mission. Astrophys J 700:1589-1602. doi:10.1088/0004-637X/700/2/1589. arXiv:0906.0766

Suzuki Y (1991) Kamiokande II results on solar neutrinos and the superkamiokande experiment. In: Fackler O, Fontaine G, Trân Thanh Vân J (eds) Massive neutrinos: tests of fundamental symmetries, Editions Frontières, Gif-sur-Yvette, p 429

Suzuki Y (1995) Kamiokande solar neutrino results. Nucl Phys B (Proc Suppl) 38:54-59. doi:10.1016/ 0920-5632(94)00733-C

Takata M, Shibahashi H (1998) Solar models based on helioseismology and the solar neutrino problem. Astrophys J 504:1035. doi:10.1086/306094

Tanner JD, Basu S, Demarque P (2013) Variation of stellar envelope convection and overshoot with metallicity. Astrophys J 767:78. doi:10.1088/0004-637X/767/1/78. arXiv:1302.5707

Tassoul M (1980) Asymptotic approximations for stellar nonradial pulsations. Astrophys J Suppl Ser 43:469-490. doi:10.1086/190678

Tassoul M (1990) Second-order asymptotic approximations for stellar nonradial acoustic modes. Astrophys J 358:313-327. doi:10.1086/168988

Thompson MJ (1993) Seismic investigation of the Sun's internal structure and rotation. In: Brown TM (ed) GONG 1992: Seismic investigation of the Sun and stars, ASP Conference Series, vol 42. Astronomical Society of the Pacific, San Francisco, p 141

Thompson MJ (1998a) Inversion methods. In: Deubner FL, Christensen-Dalsgaard J, Kurtz D (eds) New eyes to see inside the Sun and stars, IAU Symposium, vol 185. Kluwer, Dordrecht, p 125 
Thompson MJ (1998b) What we have learned from helioseismology. Astrophys Space Sci 261:23-34. doi:10.1023/A:1002068719915

Thompson MJ (2006) Magnetohelioseismology. Philos Trans R Soc Lond Ser A 364:297-311. doi:10.1098/ rsta.2005.1700

Thompson MJ, Toomre J, Anderson ER, Antia HM, Berthomieu G, Burtonclay D, Chitre SM, ChristensenDalsgaard J, Corbard T, De Rosa M, Genovese CR, Gough DO, Haber DA, Harvey JW, Hill F, Howe R, Korzennik SG, Kosovichev AG, Leibacher JW, Pijpers FP, Provost J, Rhodes EJ Jr, Schou J, Sekii T, Stark PB, Wilson PR (1996) Differential rotation and dynamics of the solar interior. Science 272:1300-1305. doi:10.1126/science.272.5266.1300

Thoul AA, Bahcall JN, Loeb A (1994) Element diffusion in the solar interior. Astrophys J 421:828-842. doi:10.1086/173695. arXiv:astro-ph/9304005

Torres-Peimbert S, Simpson E, Ulrich RK (1969) Studies in stellar evolution. VII. Solar models. Astrophys J 155:957. doi:10.1086/149924

Totsuka Y (1992) Solar and atmospheric neutrinos observed at Kamiokande. Nucl Phys B (Proc Suppl) 28:67-74. doi:10.1016/0920-5632(92)90148-L

Toutain T, Froehlich C (1992) Characteristics of solar p-modes: results from the IPHIR experiment. Astron Astrophys 257:287-297

Toutain T, Kosovichev AG (2005) Study of the solar cycle dependence of low-degree p-modes with Michelson Doppler imager and VIRGO. Astrophys J 622:1314-1319. doi:10.1086/427971

Trampedach R, Asplund M, Collet R, Nordlund Å, Stein RF (2013) A grid of three-dimensional stellar atmosphere models of solar metallicity. I. General properties, granulation, and atmospheric expansion. Astrophys J 769:18. doi:10.1088/0004-637X/769/1/18. arXiv:1303.1780

Tripathy SC, Christensen-Dalsgaard J (1998) Opacity effects on the solar interior. I. Solar structure. Astron Astrophys 337:579-590 arXiv:astro-ph/9709206

Tripathy SC, Kumar B, Jain K, Bhatnagar A (2001) Analysis of hysteresis effect in p-mode frequency shifts and solar activity indices. Solar Phys 200:3-10. doi:10.1023/A:1010318428454. arXiv:astro-ph/0103129

Tripathy SC, Hill F, Jain K, Leibacher JW (2007) Solar oscillation frequency changes on time scales of nine days. Solar Phys 243:105-120. doi:10.1007/s11207-007-9000-z. arXiv:astro-ph/0608348

Turck-Chièze S, Cahen S, Casse M, Doom C (1988) Revisiting the standard solar model. Astrophys J 335:415-424. doi:10.1086/166936

Turck-Chièze S, Basu S, Brun AS, Christensen-Dalsgaard J, Eff-Darwich A, Lopes I, Pérez Hernández F, Berthomieu G, Provost J, Ulrich RK, Baudin F, Boumier P, Charra J, Gabriel AH, Garcia RA, Grec G, Renaud C, Robillot JM, Roca Cortés T (1997) First view of the solar core from GOLF acoustic modes. Solar Phys 175:247-265. doi:10.1023/A:1004992727541

Turck-Chièze S, Couvidat S, Kosovichev AG, Gabriel AH, Berthomieu G, Brun AS, Christensen-Dalsgaard J, García RA, Gough DO, Provost J, Roca-Cortes T, Roxburgh IW, Ulrich RK (2001) Solar neutrino emission deduced from a seismic model. Astrophys J Lett 555:L69-L73. doi:10.1086/321726

Turck-chièze S, Nghiem P, Couvidat S, Turcotte S (2001) Solar internal composition and nuclear reaction rates in the light of helioseismology. Solar Phys 200:323-342. doi:10.1023/A:1010365125791

Turck-Chièze S, Couvidat S, Piau L, Ferguson J, Lambert P, Ballot J, García RA, Nghiem P (2004) Surprising sun: a new step towards a complete picture? Phys Rev Lett 93:211102. doi:10.1103/PhysRevLett.93. 211102. arXiv:astro-ph/0407176

Ulrich RK (1970) The five-minute oscillations on the solar surface. Astrophys J 162:993. doi:10.1086/ 150731

Ulrich RK (1986) Determination of stellar ages from asteroseismology. Astrophys J Lett 306:L37-L40. doi: $10.1086 / 184700$

Ulrich RK (2001) Very long lived wave patterns detected in the solar surface velocity signal. Astrophys J 560:466-475. doi:10.1086/322524

Ulrich RK, Rhodes EJ Jr (1977) The sensitivity of nonradial $p$ mode eigenfrequencies to solar envelope structure. Astrophys J 218:521-529. doi:10.1086/155705

Ulrich RK, Boyden JE, Webster L, Padilla SP, Snodgrass HB (1988) Solar rotation measurements at Mount Wilson. V. Reanalysis of 21 years of data. Solar Phys 117:291-328. doi:10.1007/BF00147250

Unno W, Osaki Y, Ando H, Saio H, Shibahashi H (1989) Nonradial oscillations of stars, 2nd edn. University of Tokyo Press, Tokyo

Valdés-Galicia JF, Velasco VM (2008) Variations of mid-term periodicities in solar activity physical phenomena. Adv Space Res 41:297-305. doi:10.1016/j.asr.2007.02.012 
Vandakurov YV (1979) Nonlinear coupling of stellar pulsations. Sov Astron 23:421

Verma K, Faria JP, Antia HM, Basu S, Mazumdar A, Monteiro MJPFG, Appourchaux T, Chaplin WJ, García RA, Metcalfe TS (2014) Asteroseismic estimate of helium abundance of a solar analog binary system. Astrophys J 790:138. doi:10.1088/0004-637X/790/2/138. arXiv:1405.7512

Vernazza JE, Avrett EH, Loeser R (1981) Structure of the solar chromosphere. III. Models of the EUV brightness components of the quiet-sun. Astrophys J Suppl Ser 45:635-725. doi:10.1086/190731

Verner GA, Chaplin WJ, Elsworth Y (2006) BiSON data show change in solar structure with magnetic activity. Astrophys J Lett 640:L95-L98. doi:10.1086/503101

Villante FL, Serenelli AM, Delahaye F, Pinsonneault MH (2014) The chemical composition of the Sun from helioseismic and solar neutrino data. Astrophys J 787:13. doi:10.1088/0004-637X/787/1/13. arXiv: 1312.3885

Vincent AC, Scott P, Trampedach R (2013) Light bosons in the photosphere and the solar abundance problem. Mon Not R Astron Soc 432:3332-3339. doi:10.1093/mnras/stt683. arXiv:1206.4315

Vitense E (1953) Die Wasserstoffkonvektionszone der Sonne. Z Astrophys 32:135

Vorontsov SV, Shibahashi H (1991) Asymptotic inversion of the solar oscillation frequencies: sound speed in the solar interior. Publ Astron Soc Japan 43:739-753

Vorontsov SV, Baturin VA, Pamiatnykh AA (1992) Seismology of the solar envelope: towards the calibration of the equation of state. Mon Not R Astron Soc 257:32-46. doi:10.1093/mnras/257.1.32

Vorontsov SV, Christensen-Dalsgaard J, Schou J, Strakhov VN, Thompson MJ (2002) Helioseismic measurement of solar torsional oscillations. Science 296:101-103. doi:10.1126/science.1069190

Vorontsov SV, Baturin VA, Ayukov SV, Gryaznov VK (2013) Helioseismic calibration of the equation of state and chemical composition in the solar convective envelope. Mon Not R Astron Soc 430:16361652. doi:10.1093/mnras/sts701

Walker G, Matthews J, Kuschnig R, Johnson R, Rucinski S, Pazder J, Burley G, Walker A, Skaret K, Zee R, Grocott S, Carroll K, Sinclair P, Sturgeon D, Harron J (2003) The MOST asteroseismology mission: ultraprecise photometry from space. Publ Astron Soc Pac 115:1023-1035. doi:10.1086/377358

Watanabe S, Shibahashi H (2001) Solar models with helioseismic constraints and the solar neutrino problem. Publ Astron Soc Japan 53:565-575. doi:10.1093/pasj/53.3.565. arXiv:astro-ph/0105445

Weiss A, Keady JJ, Magee NH Jr (1990) A collections of Los Alamos opacity tables for all temperatures. At Data Nucl Data Tables 45:209. doi:10.1016/0092-640X(90)90008-8

Weiss A, Hillebrandt W, Thomas HC, Ritter H (2004) Cox and Giuli's principles of stellar structure. Princeton Publishing, Cambridge

Winnick RA, Demarque P, Basu S, Guenther DB (2002) Seismic test of solar models, solar neutrinos, and implications for metal-rich accretion. Astrophys J 576:1075-1084. doi:10.1086/341795. arXiv:astro-ph/0111096

Woodard MF (1987) Frequencies of low-degree solar acoustic oscillations and the phase of the solar cycle. Solar Phys 114:21-28

Woodard MF, Libbrecht KG (1993) Solar activity and oscillation frequency splittings. Astrophys J Lett 402:L77-L80. doi:10.1086/186704

Woodard MF, Noyes RW (1985) Change of solar oscillation eigenfrequencies with the solar cycle. Nature 318:449-450. doi:10.1038/318449a0

Woodard MF, Libbrecht KG, Kuhn JR, Murray N (1991) Short-term changes in solar oscillation frequencies and solar activity. Astrophys J Lett 373:L81-L84. doi:10.1086/186056

Zahn JP (1992) Circulation and turbulence in rotating stars. Astron Astrophys 265:115-132

Zhang QS (2014) The solar abundance problem: the effect of the turbulent kinetic flux on the solar envelope model. Astrophys J 787:L28. doi:10.1088/2041-8205/787/2/L28. arXiv:1404.4996

Zweibel EG, Gough D (1995) Is there a seismic signature of the Sun's magnetic field? In: Hoeksema JT, Domingo V, Fleck B, Battrick B (eds) Helioseismology, ESA Special Publication, vol SP-376. ESA, Noordwijk, p 73 